\title{
Membrane mechanics governs cell mechanics in epithelial cell: how surface area regulation ensures tension homeostasis
}

\author{
Dissertation \\ zur Erlangung des mathematisch-naturwissenschaftlichen Doktorgrades \\ "Doctor rerum naturalium" \\ der Georg-August-Universität Göttingen \\ im Promotionsprogramm Chemie \\ der Georg-August University School of Science (GAUSS) \\ vorgelegt von \\ Anna Pietuch \\ geboren in Sokolow / Malopolski, Polen
}

Göttingen, 2012 


\section{Betreuungsausschuss}

Prof. Dr. Andreas Janshoff

Institut für Physikalische Chemie

Georg-August-Universität Göttingen

Prof. Dr. Jörg Großhans

Zentrum Biochemie und Molekulare Zellbiologie

Abtl. Entwicklungsbiochemie

Universitätsmedizin Göttingen

\section{Mitglieder der Prüfungskommission}

Referent: $\quad$ Prof. Dr. Andreas Janshoff , Institut für Physikalische Chemie, Georg-August-Universität Göttingen

Korreferent: Prof. Dr. Jörg Großhans, Zentrum Biochemie und Molekulare Zellbiologie Abtl. Entwicklungsbiochemie, Universitätsmedizin Göttingen

Weitere Mitglieder der Prüfungskommission

Prof. Dr. Mikael Simons

Max-Planck-Institut für experimentelle Medizin

Prof. Dr. Sarah Köster

Institut für Röntgenphysik/ Courant Research Centre

Dr. Florian Rehfeldt

Drittes Physikalisches Institut - Biophysik, Georg-August Universität Göttingen

Prof. Dr. Marina Bennati

Max-Planck-Institut für Biophysikalische Chemie,

Tag der mündlichen Prüfung: 07.12.2012 
Den Menschen die ich liebe

„Geh nicht nur die glatten Straßen.

Geh Wege, die noch niemand ging, damit du Spuren hinterlässt und nicht nur Staub“. Antoine de Saint-Exupéry 



\section{Content}

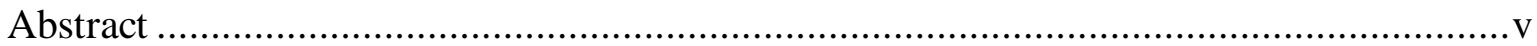

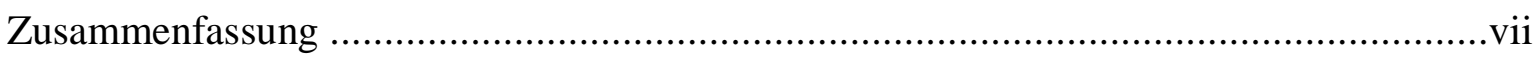

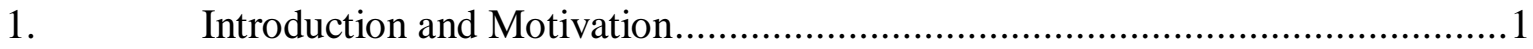

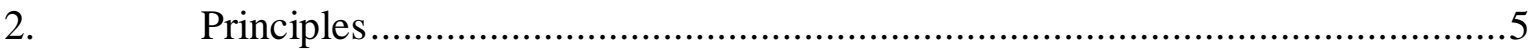

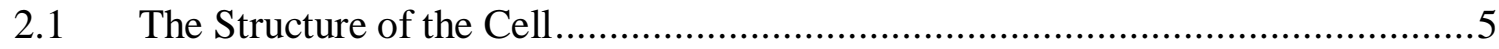

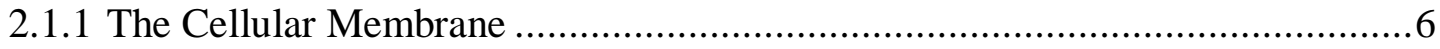

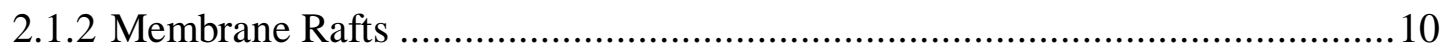

2.1.3 Membrane-Cytoskeleton Adhesion ............................................................10

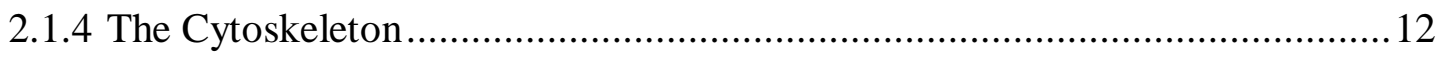

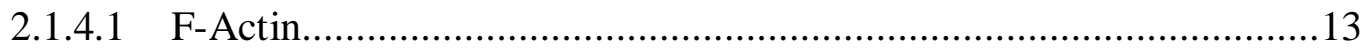

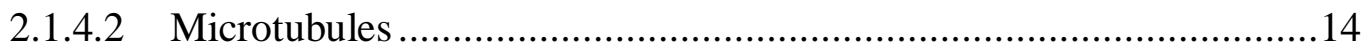

2.1.4.3 Intermediate Filaments .................................................................15

2.1.4.4 Mechanical Properties of the Cytoskeleton Components...................16

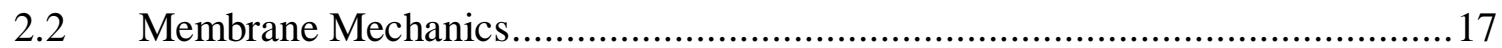

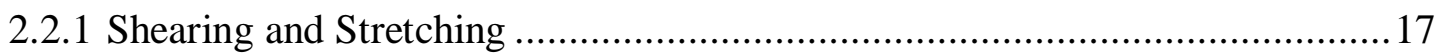

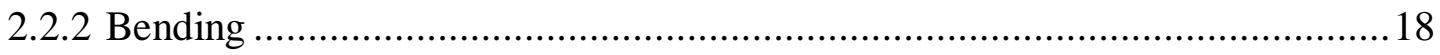

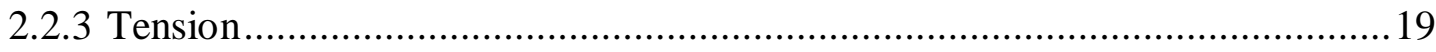

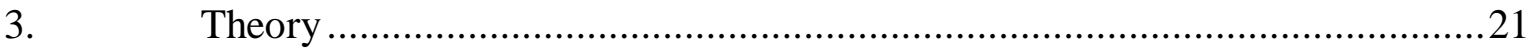

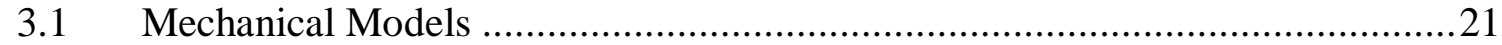

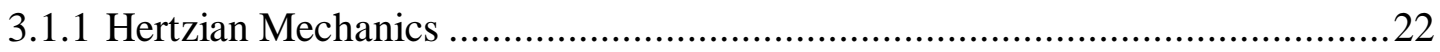

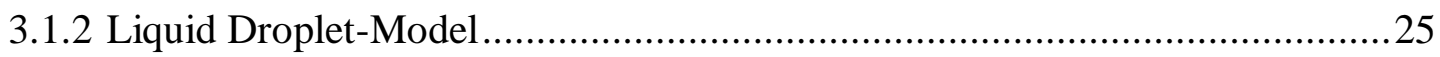

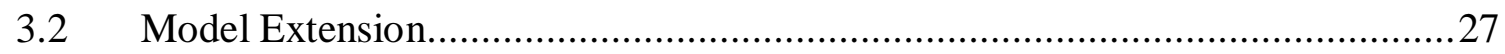

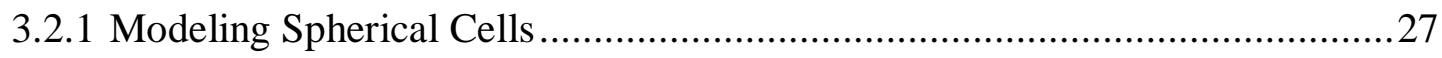

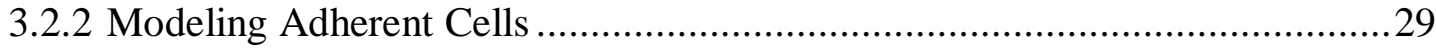

3.2.3 Geometrical Description of Adherent MDCK II Cells ..................................31

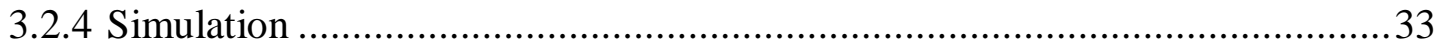

3.3 Mechanical Description of Pulled Nanotubes..................................................35

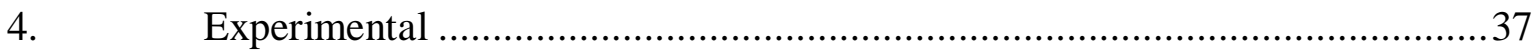

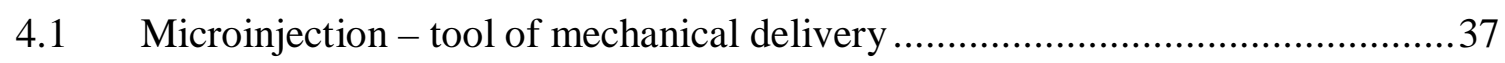

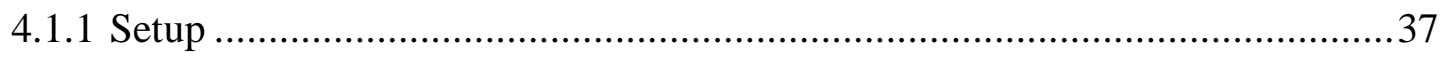

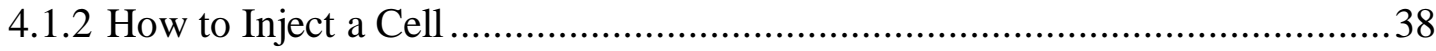

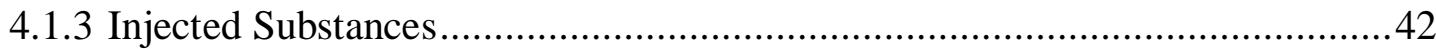




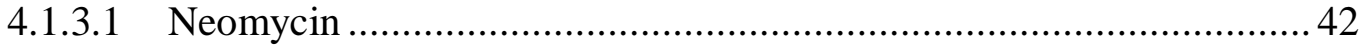

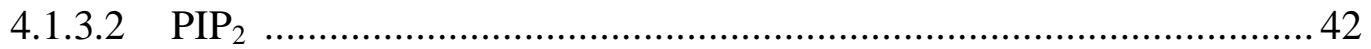

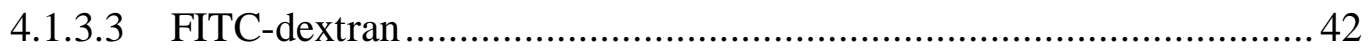

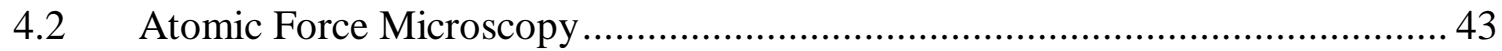

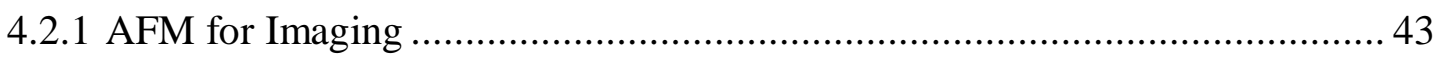

4.2.2 AFM for Elasticity Measurements …........................................................ 46

4.2.3 AFM for Probing Tether Forces................................................................. 48

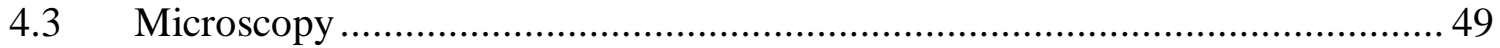

4.3.1 Phase Contrast Microscopy …...................................................................... 49

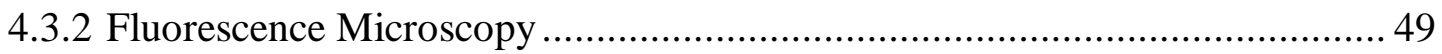

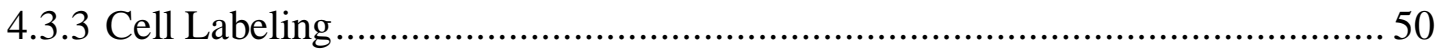

4.3.4 Scanning Electron Microscopy ................................................................. 52

$4.4 \quad$ Electric Cell-Substrate Impedance Sensing................................................. 52

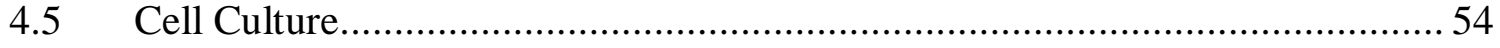

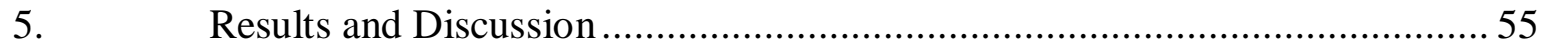

5.1 Mechanical Properties of confluent MDCK II Cells ......................................... 55

5.1.1 Structural Characterization of MDCK II Cells ............................................. 55

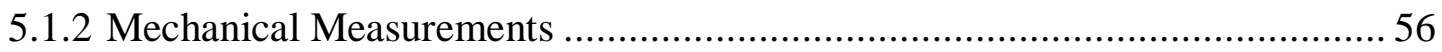

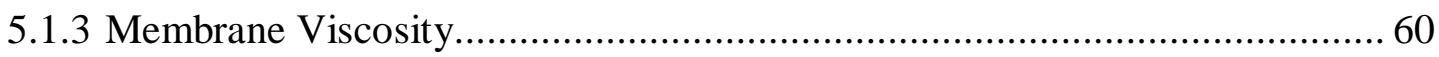

5.1.4 Bending Rigidity and Tether Radius ...................................................... 61

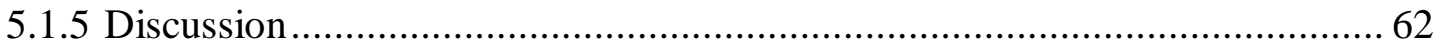

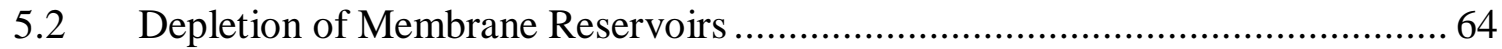

5.2.1 Morphological Alteration During Cholesterol Depletion............................... 64

5.2.2 Mechanical Changes Due to loss of Membrane Reservoir............................. 70

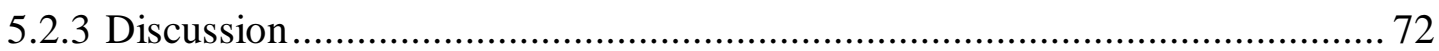

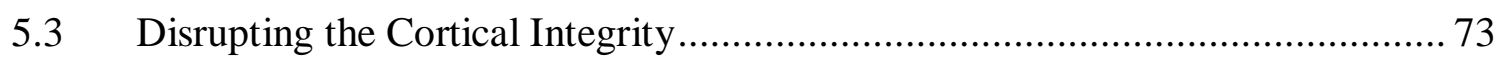

5.3.1 Disassembly of F-actin with Cytochalasin ................................................ 73

5.3.2 Disrupting Actomyosin Connections with Blebbistatin ................................. 74

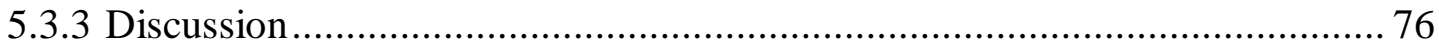

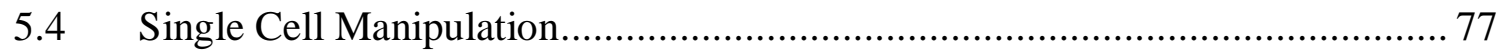

5.4.1 Uncoupling the Membrane from the Cytoskeleton...................................... 77

5.4.1.1 Morphological Alteration after Neomycin Injection ......................... 77

5.4.1.2 Mechanical Properties of Neomycin Injected Cells .......................... 79

5.4.1.3 Cell Stiffening Depends on Ezrin Disappearance ............................. 81 
5.4.1.4 Further Reactions of Neomycin Injected Cells................................83

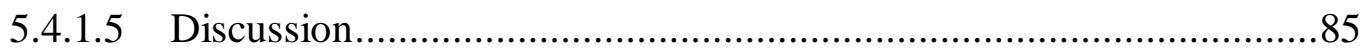

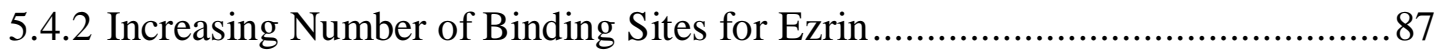

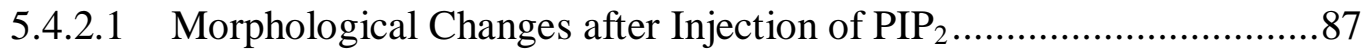

5.4.2.2 Mechanical Investigation of $\mathrm{PIP}_{2}$ Injected Cells .............................89

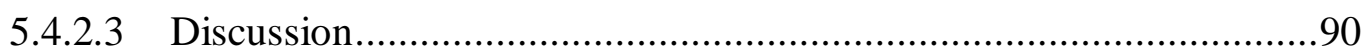

5.4.3 Injection of FITC-dextran - proof of principle ..........................................91

5.5 Mechanics of Cell Membrane Fragments ...................................................... 93

5.6 Osmotic Challenge to the Cellular Membrane............................................... 97

5.6.1 Shape Changes during Osmotic Stress .....................................................98

5.6.2 Mechanical Response to Hypoosmotic Stress............................................... 101

5.6.3 Cells Regulate Membrane Tension/Area during Prolonged Hypoosmotic

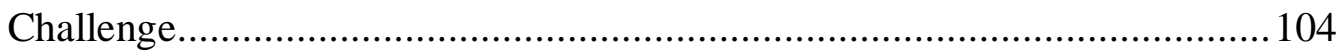

5.6.4 Hyperosmotic Shock: From Liquid Droplet to a Drum like Geometry..........109

5.6.5 Cellular Reaction on Distilled Water........................................................ 111

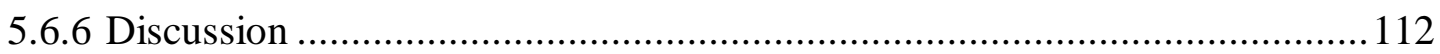

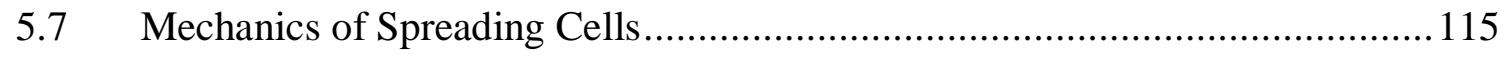

5.7.1 Changes in Cellular Mechanics during Cell Spreading ................................116

5.7.2 Morphological Changes during Adhesion and Spreading ...........................117

5.7.3 Mechanical Models - Change from Sphere to Caped Sphere........................118

5.7.4 Mechanical Parameter during Adhesion and Spreading ..............................120

5.7.5 Spherical Cell- Before Adhesion and Spreading ........................................122

5.7.6 First and Second Phase of Cell Spreading ............................................... 124

5.7.7 Inhibition of Adhesion Abolishes Tension Drop..........................................126

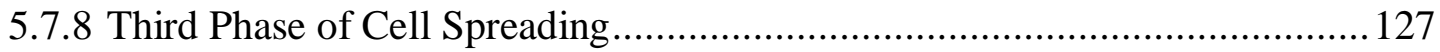

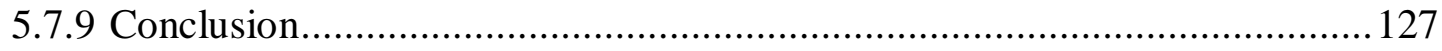

6. Final Discussion and Concluding Remarks ...........................................129

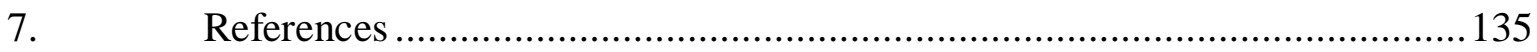

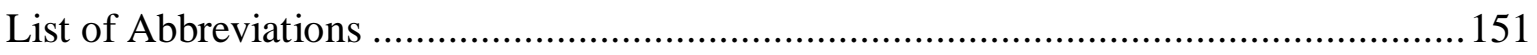

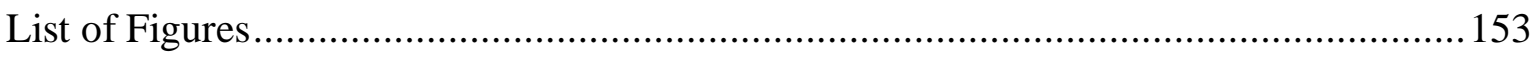

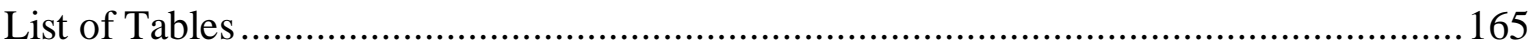




\section{Abstract}

The plasma membrane tension in eukaryotic cells is supposed to be a major regulator of many cellular processes that involve changes in membrane area and membrane deformation including cell migration, mitosis, endo -and exocytosis, membrane repair, osmoregulation, and cell spreading. The goal of this study is to investigate how polar epithelial cells adjust their surface area in response to tension changes induced by external stimuli that modulate cytoskeleton-membrane attachment, cytoskeleton integrity, cholesterol content, and morphology of the apical membrane. Here, confluent MadinDarby canine kidney cells (MDCK II) were used to investigate tension-controlled cellular surface area regulation. Indentation experiments combined with pulling of membrane tethers carried out with an atomic force microscope (AFM) were used to simultaneously assess local changes in membrane tension and excess area as a function of external stimuli. Chemical stimuli comprise interference with the functionality of the actomyosin cortex using blebbistatin, cytochalasin $\mathrm{D}$ and the disassembly of cytoskeleton-membrane attachment sites via single cell injection of neomycin to abolish binding of ERM proteins to phosphatidylinositol(4,5)-bisphosphate $\left(\mathrm{PIP}_{2}\right)$. To increase the number of binding sites for ezrin $\mathrm{PIP}_{2}$ is microinjected into single cells. Furthermore, microvilli are cleared off by cholesterol-extraction from the plasma membrane or the cells are challenged by different osmotic solutions to observe changes in membrane tension with respect to membrane tension buffering reservoirs. Additionally, the elastic properties of apical cell membrane sheets derived from confluent MDCK II cell monolayers are determined in the absence of the cell interior and devoid of the cytoskeleton. Finally, the mechanical changes encompassing cell adhesion to the substrate and spreading are investigated. In conclusion, the plasma membrane consisting of a phospholipid bilayer is laterally inextensible due to its liquid-crystalline nature thereby producing restoring forces in response to deformation. This renders certain cellular tasks such as motility and adaption to osmotic stress challenging with respect to lysis. A solution for this problem is the presence of highly dynamic membrane reservoirs such as microvilli with its rapid accessibility controlled by homeostasis of lateral tension. Here, the plasma membrane's in-plane tension and the available surface area were measured simultaneously in a precise and local fashion using either confluent cell monolayers or single cells. The experiments allow us to draw a comprehensive picture of how surface area regulation is reflected in membrane mechanics. 


\section{Zusammenfassung}

Die Plasmamembranspannung von eukaryotischen Zellen soll maßgeblich zur Regulation von zellulären Prozessen wie der Zellmigration, Mitose, Endo- und Exozytose, Membranreparatur, Osmoregulierung und Zellspreiten beitragen, welche zu einer Veränderung der Membranfläche und ihrer Deformation führt. In dieser Arbeit wurde die epitheliale Zelllinie MDCK II (Madin-Darby Canine Kidney) benutzt, um spannungsgesteuerte Oberflächenregulierung zu untersuchen. Indentationsexperimente kombiniert mit dem Herausziehen von Membrannanoröhren wurden mit Hilfe des Rasterkraftmikroskops (Atomic Force Microskope, AFM) durchgeführt, um lokale Variationen in der Membranspannung und überschüssiger Membranfläche als Funktion von äußeren Reizen abzuschätzen. Die verwendeten externen Stimuli beinhalten eine Veränderung der Funktionalität des Actomyosin-Cortexes durch die Wirkung von Blebbistatin und Cytochalasin D, sowie die Manipulation der Zytoskelett-Membran Adhäsionspunkte durch Einzel-Mikroinjektion. Die Injektion von Neomycin verhindert die Anbindung von ERM-Proteinen an das Lipid Phosphatidylinositol-(4,5)-bisphosphat ( $\left.\mathrm{PIP}_{2}\right)$ und bewirkt somit die Abkopplung des Zytoskeletts von der Plasmamembran. Als Gegenexperiment diente die Injektion des Lipids $\mathrm{PIP}_{2}$ selbst, welches zur Erhöhung der Anzahl der Zytoskelett-Membran Adhäsionspunkte führte. Weiterhin wurden die als Membranreservoire dienenden Mikrovilli durch den Entzug von Cholesterol entfernt. Auswirkung auf das Vorhandensein von Membranreservoiren hat ebenfalls die Veränderung des osmotischen Drucks innerhalb der Zellen. Zusätzlich wurden die elastischen Eigenschaften von apikalen Zellmembran-Fragmenten von konfluenten MDCK II Zellen untersucht, welche Aufschluss über die intrinsischen Membraneigenschaften ohne den Einfluss des Zytosols und Zytoskeletts geben konnten. Abschließend wurde die Mechanik von adhärierenden und spreitenden Zellen untersucht. Zusammenfassend kann gesagt werden, dass die Plasmamembran, bestehend aus einer Phospholipiddoppelschicht, lateral schwer ausdehnbar ist aufgrund ihrer flüssig-kristallinen Natur. Durch das Vorhandensein von dynamischen Membranreservoiren wie Mikrovilli, die schnell auf Veränderungen der Membranspannung durch Membranhomöostase reagieren, werden zellulare Prozesse wie die Zellmotilität oder die Anpassung an osmotischen Stress ermöglicht. In der vorliegenden Arbeit gelang es gleichzeitig, die Membranspannung und die Verfügbarkeit von Membranfläche von adhärenten konfluenten als auch von adhärierenden und spreiten Zellen zu messen. Die durchgeführten Experimente ergaben ein 
detailliertes Bild wie sich die zelluläre Oberflächenregulierung in der Membranmechanik widerspiegelt. 


\section{Introduction and Motivation}

The interdisciplinary field of cellular mechanics combines scientific attainments from biology, chemistry and physics. It deals with forces exerted or experienced by cells or tissues and analyzes molecular mechanisms underlying the cellular response to mechanical signals. Cells resist dynamically to deformation, the way how is described by the mechanical properties of the cells. These specific characteristics are determined by the cells' composition and internal structures as well as the environment. Alteration of the principle elements of the cellular structure can lead to variation in the mechanical features and breakdown of physiological functions. In the last decades, various studies describe the correlation of changes in cellular mechanics and diseases. Particular attention is paid to the mechanical alteration found for cancer cells [1]. Although the physical change is addressed as the end-result of a disease progress, recent advances in mechanobiology reveal that alteration in cell and tissue response to mechanical stress actively contribute to disease development [2]. When studying cellular mechanics the fundamental and intrinsic mechanical properties of the plasma membrane have to be considered. The mechanical characteristics rely on the chemical nature of the membrane components and the forces acting between them. The mechanical behavior of the membrane is largely governed by the membrane tension. The plasma membrane tension in eukaryotic cells is supposed to be a major regulator of many cellular processes that involve changes in membrane area and membrane deformation including cell migration [3], mitosis [4], endo-and exocytosis [5] [6], membrane repair [7], osmoregulation, and cell spreading [8] [9]. The overall plasma membrane tension encompasses the in-plane tension of the lipid bilayer, the membranecytoskeleton adhesion and is actively controlled by the contractile actomyosin cortex [10]. The cytoskeleton as an internal scaffold consists of a complex network of three biopolymers (F-actin, microtubules and intermediate filaments) that together with associated proteins provides the mechanical stability of the cells and thereby determines the cell's shape. A prominent representative of proteins closely related with the plasma membrane architecture are proteins from the ERM-(ezrin-radixin-moesin) family [11] [12]. ERM proteins accumulate underneath the plasma membrane forming cell structures such as membrane ruffles, cell-cell contacts and microvilli [13] [14]. The proteins are expressed tissue-specfic whereby many epithial cell expressing ezrin [15]. In the active state, the N- 
terminal domain of the protein interacts with the membrane, e.g. phosphatidylinositol-4,5bisphosphat $\left(\mathrm{PIP}_{2}\right)$ [16] and the C-terminus is responsible for binding F-actin.

The intricate interplay between the plasma membrane and its cortex enables cells to withstand mechanical challenges posed by the environment. As membranes are largely inextensible and cannot bear large strains due to the liquid-crystalline nature (critical area dilatation is limited to 3-4 \%) [17], regulatory processes to maintain the overall plasma membrane tension below lysis tension are required, more precisely, tension-driven surface area regulation is necessary to accommodate changes in tension. To provide sufficient membrane area, cells store excess membrane in reserves like protrusions (microvilli) and invaginations (caveolae), which by virtue of unfolding buffer membrane tension to accommodate 20-30\% stretching [18] [19] [20]. A physiologically relevant mechanical stimulus is osmotically induced stress since animal cells have to sustain substantial fluctuations in the osmolarity of external fluids, which produces considerable pressure differences between the cytosol and the environment. Osmotic pressure forces the cell to quickly adapt in order to avoid damages of the inextensible plasma membrane. When cells are subjected to hypoosmotic solution they increase their volume and subsequently their surface area. Animal cells can increase their surface area by a factor of 3 and ten times their volume, depending on the cell type [21]. The deformation and supply of excess membrane are key factors in cellular adhesion and motility. Lymphocytes migrating through an intact cellular layer rely on the ability to adhere and move through tissue [22]. During adhesion and spreading, for example after cell division, the plasma membrane has mechanically to deform from a spherical to a nearly discoidal form. Stretching an epithelial cell causes the cell to withdraw lateral protrusions while suppression of spreading activity is reversed upon release of tension [23]. Chemically induced reduction of membrane tension leads to faster cell spreading [9], promoting the idea that membrane mechanics, i.e. the in-plane tension, limits important cellular processes.

The conventional strategy to determine membrane tension is to use the micropipette aspiration technique either stand-alone or in combination with laser tweezers. While these methods depict the mechanical situation of cells in suspension in an appropriate way, adherent cells are barely described. The atomic force microscope is a powerful tool to examine small forces in the range of $\mathrm{pN}$. By applying suitable mechanical models this technique is also capable to measure the tension of the plasma membrane of both spherical 
[24] and adherent cell [25]. Here, it is assumed that the cells behave as a liquid droplet with an isotropic in-plane tension. Informations about confluent epithelial cells establishing a tissue are scarce. In the present work an extended mechanical approach for the investigation of adherent and confluent cells is proposed and validated. This model is based on the liquid droplet theory, equipping the cells with a cortical surface tension, since the membrane underlying cortex exerts tension to the membrane. The main goal of the study is to investigate the cellular response to rearrangements of the cortical cytoskeleton structure underlining the plasma membrane. In detail, the cells will be subjected to various stimuli targeting membrane reservoir availability, actomyosin-integrity, membrane cytoskeleton attachment sites through injection of neomycin and $\mathrm{PIP}_{2}$ and hydrostatic pressure provoking a tension-driven response in the available surface area. These experiments are devised to govern a deeper insight into the mechanics of the plasma membrane and to learn how the adhesion of the cytoskeleton to the membrane contributes to it. 


\section{Principles}

\subsection{The Structure of the Cell}

Having a closer look inside the cell, the drawing from Figure 1 does not reflect all the details, in particular all molecules and structures, essential for life. The cell interior is heavily crowded as illustrated by David Goodsell in his book "The machinery of life” [26], capturing the beauty and complexity of the cellular structure. We will be satisfied by a more schematic approach to explain the most relevant features in terms of the cellular structure and cellular mechanics.

Cells can be divided into two classes regarding their internal design. The first group contains prokaryotic cells characterized by lack of internal membranes. Representatives are mycoplasmas and bacteria. The second category includes eukaryotic cells, known for their intracellular compartmentalization such as plant and animal cells. Eukaryotes use internal membranes to further separate the inner content (organelles) and provide additional active surface inside the cell. This work solely focuses on animal cells, especially cells derived from epithelia. The most important organelles of animal cells are the following [27]:

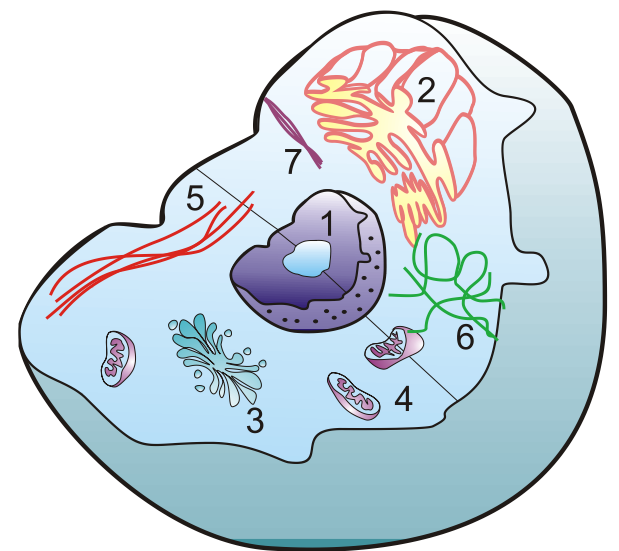

Figure 1: Schematic drawing of a eukaryotic cell.

(1) Nucleus: Contains the major of the cells' genetic information (DNA). It is surrounded by a double membrane equipped with pores allowing to passage material.

(2) Endoplasmatic reticulum: The rough ER surrounds the nucleus and shows a large surface because of a series of folded membrane sheets, on which proteins are synthesized by ribosomes. 
(3) Golgi apparatus: Membrane enclosed disc like organelle and the sorting place for proteins. Small vesicles dispatch and transport proteins to regions in the cell interior.

(4) Mitochondria: Consist of a double membrane and produces the cells' energy currency ATP.

The whole cell interior, without the nucleus, is termed the cytoplasma, including organelles and the aqueous cytosol. Structural stability of the cell body is achieved by the cytoskeleton. It is a network of filaments (Fig. 1: F-actin (5), microtubules (6), intermediate filaments (7)), drawing through the entire cell, shaping the cell membrane and providing mechanical stability. Cellular cargos are transported along these filaments [28], except the intermediate filaments. Vesicle trafficking plays a crucial role in the formation and maintenance of different intracellular compartments as well as in the communication between cells and their environment [29]. Moreover, endo- and exocytosis plays a fundamental role in membrane homeostasis [17]. For a more detailed description of cellular processes please refer to biochemical textbooks [30]. In the following subsections the intricate interplay between cellular structures and mechanical characteristics of the cellular plasma membrane will be described.

\subsubsection{The Cellular Membrane}

The cellular plasma membrane owns specific properties to carry out its function. Forming a stable barrier between the cellular interior and the outside, the plasma membrane with its structural depending abilities and embedded proteins allows selective transport of molecules and ensures cell-cell recognition [30].

Cellular membranes mainly consist of lipids and proteins, at different composition changing the fluidity and lending the membrane its special properties. In numbers, there are roughly $10^{9}$ lipid molecules forming the plasma membrane of an animal cell [30] [31]. The most commonly occurring lipids are phospholipids, glycolipids and cholesterol. Phospholipids contain a glycerol-backbone, a tail consisting of two fatty acid chains (in animal membrane between 16 and 18 carbons) and a phosphorylated alcohol representing the hydrophilic head (glycerol, ethanolamine, choline, serine and inositol ) (Figure 2).

There is an asymmetry in the distribution of lipids within the plasma membrane. Nearly all anionic lipids in eukaryotic cells face the cytoplasm, whereas lipids with large glycosylated 
head groups are exposed to the extracellular environment [32]. There are differences between the membrane asymmetry in eukaryotic and prokaryotic cells. Prokaryotic cell membranes possess more negatively charged lipids on the outer membrane. This difference in membrane composition explains the function of antibiotics. For example antimicrobial peptides, serving as a first line of defense against infection, bind particularly anionic lipids [33]. A relevant membrane asymmetry is found in the inner leaflet of the plasma membrane in animal cells were, e.g. the phospholipid phosphatidylinositol, is concentrated. There are lipid kinases which phosphorylate inositol phospholipids and hence activating signal pathways leading to cell growth, cell cycle entry, cell migration, and cell survival [34] [35].

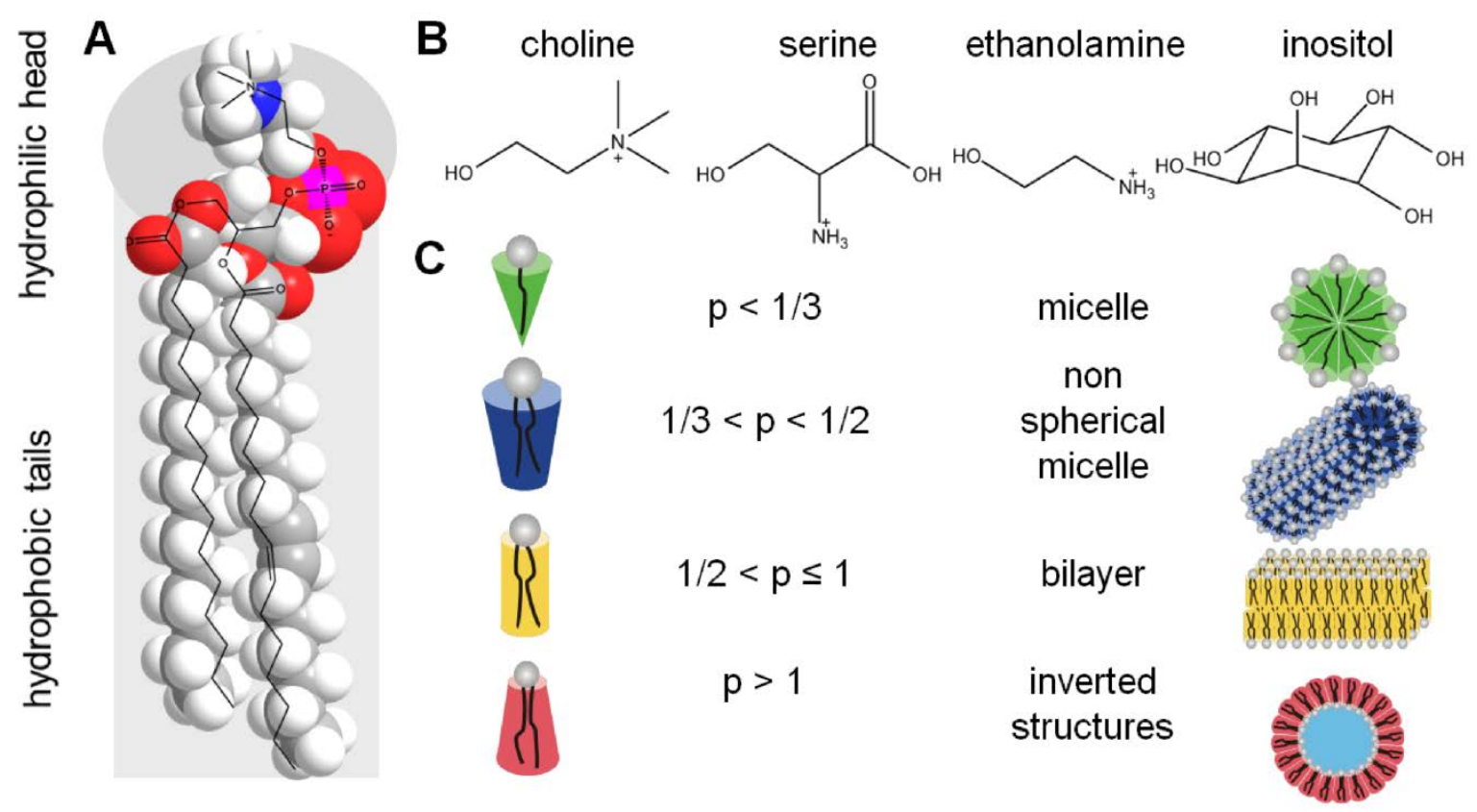

Figure 2: A: Calotte model overlaid with chemical structure of a phospholipid, highlighted are head and tail group to emphasize amphiphilic character. B: Chemical structure of most common alcohols building the hydrophilic head of a lipid. C: Packing parameter $p$ predicts the structure of the aggregates formed from lipids placed in aqueous solution.

The thickness of a phospholipid bilayer is about 4-5 $\mathrm{nm}$. The spontaneous curvature and fluidity depends on the length of the carbon chains, the size of the head groups and the mixing ratio of different lipids and sterols. Cholesterol provides the plasma membrane with more rigidity and offers a more diverse lateral organization constituting signaling nanodomains in conjunction with sphingolipids. The head group of cholesterol is small and 
the bulky tail with its rigid ring structure immobilizes the fatty acid chains of the phospholipids of the bilayer, becoming less deformable (Figure 3).

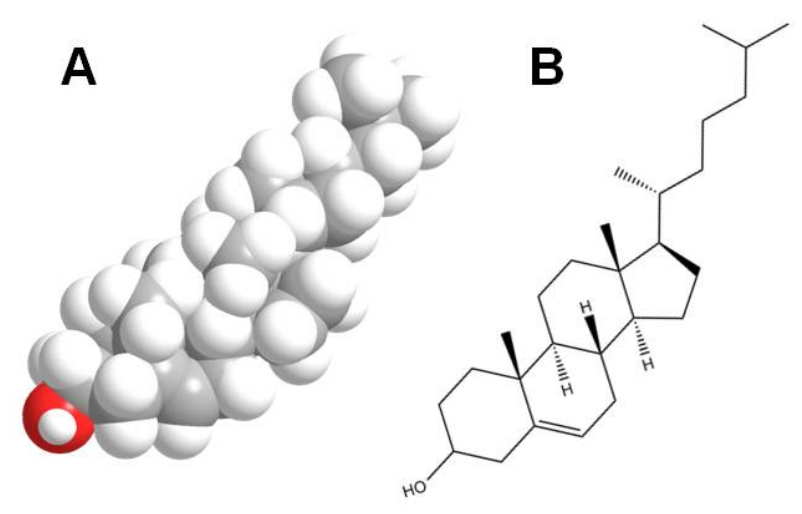

Figure 3: Chemical structure of cholesterol A: Calotte model. B: Structural formula.

Having a hydrophilic head group and a hydrophobic tail, phospholipids display an amphiphilic character. When amphiphilic molecules are placed in water, they spontaneous self assemble to aggregates like micelles, vesicles and bilayer. This aggregation is driven by the hydrophobic effect, which describes the behavior of non-polar molecules in contact with water. The strong tendency of water molecules to form $\mathrm{H}$-bond with each other, determine the interaction with non-polar molecules incapable of forming H-bonds like alkanes and hydrocarbons. The reorientation of water molecules around hydrophobic solutes is entropically unfavorable, since it disrupts the existing water structure. A cage of highly ordered water molecules forms around the lipids that increase entropy loss. By aggregating together, non-polar molecules diminish this loss of entropy through minimizing the surface area exposed to water. This segregation of non-polar molecules and water leads to spontaneous self assembly of diverse aggregates. Regarding lipids, the shape of these aggregates depend on the geometry of the individual molecules [36].

Israelachvilli proposes a packing parameter $p$ defined as $V \cdot(a l)^{-1}$, were $V$ is the volume, $a$ the surface of the lipid and $l$ the length of the tail to explain the various type of aggregates. Depending on the geometry of the lipid molecules, one of the in Figure 2 shown structures will form. Because of their cylindrical shape, phospholipids forms bilayer. In this energetic stable state the head groups are facing the water site, hence shielding the hydrophobic tails inside the bilayer. Once the bilayer is formed, it will be stabilized by van der Waals forces (attractive forces and hydrophobic interactions between the phospholipid tails) and 
additionally hydrogen bonding and electrostatic interaction (between the polar head groups and the water molecules). The interactions between the lipids are not covalent, therefore allowing a high lateral diffusion with a diffusion coefficient of $D=10^{-8} \mathrm{~cm}^{2} \mathrm{~s}^{-1}$ [30].

The onset of micelle formation is referred to as the critical micelle concentration (CMC). Increasing the concentration of the amphiphile above the CMC enhanced the number of micelles but not the average size. Double chained lipids, as the phospholipids building the cellular membrane show a very low CMC of less than $10^{-12}$ molar. This means that the material is used very efficiently by the cell to establish a bilayer as boundary between cell interior and the environment and remains intact even at low concentration of lipids [27].

The plasma membrane of cells includes more than an arrangement of lipids. It embedded diverse membrane proteins and together they construct a complex active system governing diverse biological process as the transport of compounds across the membrane by channels and pumps, cell-cell recognition by carbohydrates tethered to the membrane, signal cascades triggered by the binding of proteins to membrane receptors and endo-and exocytosis. These events require dynamic membrane regions allowing fast assembly and disassembly of structures like the cortical cytoskeleton which is underlying and thereby stabilizing the membrane. 


\subsubsection{Membrane Rafts}

In 1997 Simons and Ikonen introduced the "lipid raft" concept, where the postulated sphingolipid-cholesterol-protein assembly plays a key role in membrane trafficking and signaling [37]. This concept attributes lipids a special function by introducing chemical specificity into the plasma membrane [38], and exempts lipids from the passive feature as fluid solvent [39]. However, rafts are short-timed and difficult to visualize in living cells and therefore several investigators question the existence of this membrane domains [40].

After more than 10 years of intensive investigation of "lipid rafts" new microscopic techniques reveal convincing data on the existence of membrane rafts [41], leading to a new definition of "lipid rafts": "Membrane rafts are small (10-200 nm), heterogeneous, highly dynamic, sterol- and sphingolipid-enriched domains that compartmentalize cellular processes. Small rafts can sometimes be stabilized to form platforms through proteinprotein and protein-lipid interaction” [42]. The regulatory phospholipid $\mathrm{PIP}_{2}$ is enriched in cholesterol-depending membrane domains [43] [44] [45]. $\mathrm{PIP}_{2}$ directly regulates the actin cytoskeleton by modulating actin regulatory proteins [46], leading to a pivotal role of this lipid in organizing the cellular cortex [47].

\subsubsection{Membrane-Cytoskeleton Adhesion}

The capability of cells to organize specific regions of their plasma membrane like membrane rafts is of great importance. In order to coordinate dynamic processes at the plasma membrane like endo- and exocytosis or cell division, the cell has to dynamically remodel the membrane-cytoskelton-adhesion. A prominent representative of proteins closely related with the plasma membrane architecture is the ERM-( ezrin-radixin-moesin) family. ERM proteins that accumulate underneath the plasma membrane are essential for the formation of cell structures as membrane ruffles, cell-cell contacts and microvilli [48]. The organization of these structues require a precise interplay of the cellular membrane, membrane-associated proteins and the cytoskeleton. The ERM-proteins are expressed in a tissue-specfic manner, with many epithial cell expressing ezrin [15] and endothial cell mainly moesin [48]. Together with the results from Woodward et al., where they showed that MDCK cells express a high level of ezrin and only a low level of radixin and moesin 
[49], the focuss of this study lies in membrane-cytoskeleton adhesion via ezrin and its impact on the mechanical properties of the plasma membrane of MDCK II cells.

Ezrin consists of a 300 amino acid long plasma membrane associated FERM (four point one, ezrin, radixin, moesin) domain at the end of the amino terminus (Figure 4). The ERMassociated domain (C-ERMAD) is located at the C-terminus, able to either bind both the FERM-domain or F-atin [50]. This enables the protein to adopt a dormant state in which the C-ERMAD is associated with the FERM-domain [51] [52].

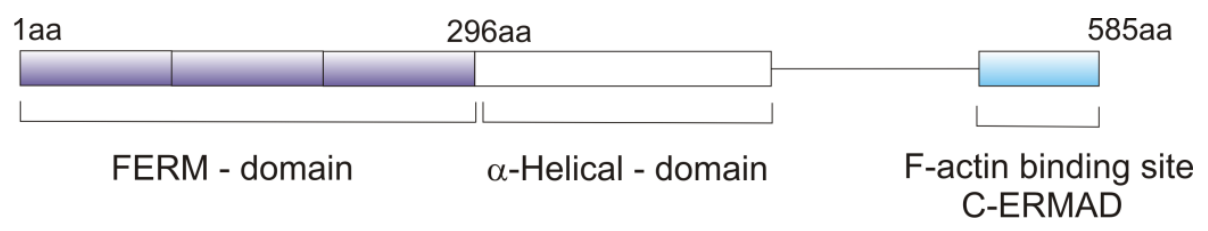

Figure 4: Domain organization of the protein ezrin.

Activation and opening of the inactivated, masked proteins is due to phosphorylation of the Thr-567 residue, located at the C-terminus and hence the F-actin binding site [53] [54]. Several studies have shown that $\mathrm{PIP}_{2}$, localized at the inner leaflet of the plasma membrane, also appears to be binding partner for ezrin (Figure 5) [55]. Later a two step model for the activation of ezrin was proposed [56]: ERM proteins are recruited from a cytoplasmatic pool of dormant molecules to $\mathrm{PIP}_{2}$ rich regions at the plasma membrane, followed by a phosphorylation step and F-actin binding.

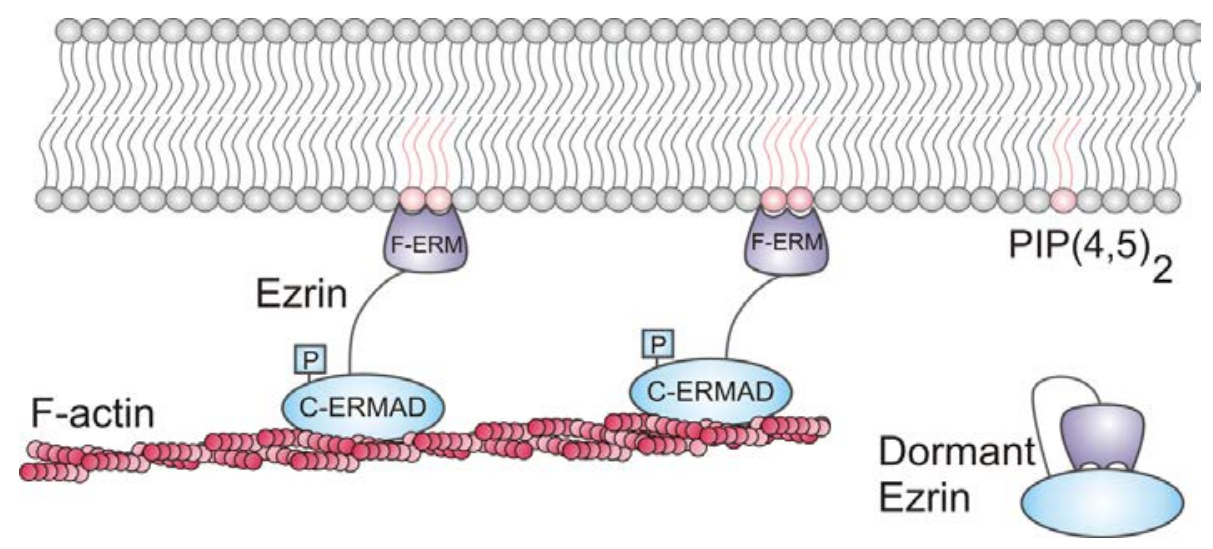

Figure 5: Schematic illustration of ezrin binding to the plasma membrane and the F-actin cytoskeleton.

Intensive research is currently carried out to elucidate ezrin's contribution to cell signaling, bringing ERM-proteins and the activation of RhoA, a small GTPase regulating cortical actin cytoskeleton, together into context [48]. Besides its role as membrane-cytoskeleton anchor ezrin is also very closely involved in the dissemination and growth of cancer. It 
coordinates and amplifies metastasis-associated cell-surface signals and alters the intracellular signaling cascade [57]. This shows the necessity to study the close connection of cellular mechanics and membrane-ezrin-cytoskeleton organization, as mechanical properties of cancer cells are strongly altered [1]. Oncologists often diagnose cancer due to changes of tissue stiffness sensed by palpation. Because of a stiffer extracellular matrix, tumors are more rigid than the surrounding tissue [58]. Numerous studies describe the contrary observation, where cancerous cells are more deformable (lower stiffness) than normal cells, often due to reorganization of the cytoskeleton [1]. Until now great effort is made to gain a detailed understanding of the mechanisms of cancer progression and invasiveness and its connection to cell mechanics. However, the contribution from the cytoskeleton as internal scaffold to changes in the mechanical properties of the cells is undisputed.

\subsubsection{The Cytoskeleton}

The cytoskeleton as the dominant internal scaffold comprises a complex network of three classes of biopolymers, giving the cellular membrane mechanical stability and thereby shaping it. The cytoskeleton determines many important and divers functions inside the cell: During cell division, the cytoskeleton pulls the chromosomes apart (mitosis); afterwards it separates the dividing cell into two. Along the filaments cargos and organelles are guided through one part of the cell to another. The cytoskeleton filaments are also responsible for different types of movement, inducing swimming with structures formed like propellers (sperm cell, Helicobacter pylori), but also for surface mediate moving like crawling of blood cells or fibroblasts across a matrix. The cytoskeleton also provides the machinery inside the muscle cells to coordinate contraction [30]. The way how these complex networks are arranged, whether dense woven or partly disassembled or cross linked, affect the cellular functions together with the cellular mechanics.

Regulation of the assembly and disassembly of the cytoskeleton filaments enables eukaryotic cells to build up complex filamentous structures and in case of need (movement or cell division) to degrade it. Many biological polymers like DNA, RNA and many other proteins are held together by covalent bonds. However, the cytoskeleton relies only on weak non-covalent interactions, explaining the rapid assembly and disassembly of these structures. 


\subsubsection{F-Actin}

The actin monomer is a single globular polypetide. The monomers assemble head-to-tail, in a ATP-hydrolysis driven process, building filaments with structural polarity. The actin filaments consist of two parallel protofilaments, which are wound around each other to from a helix (Figure $6 \mathrm{~A}$ ). The actin polymerization starts at the plasma membrane. Therefore, filamentous actin is primarily found at the cell periphery [30]. The nucleation is controled by actin related proteins called Arp 2/3-complex [59]. This complex also induce the formation of the treelike web found directly under the plasma membrane, the so-called cortex. To prevent an uncontrolled polymerization of globular actin monomers (G-actin) from the cytosol, the protein thymosin binds G-actin and sterically inhibits filament elongation at the puls-end. Recruitment of actin monomers from this polymerizationinactiv-pool is due to the interaction to antoher monomer-binding-proteins, profilin. The profilin-actin complex can add to the free plus end of a growing filament and promotes filament elongation.
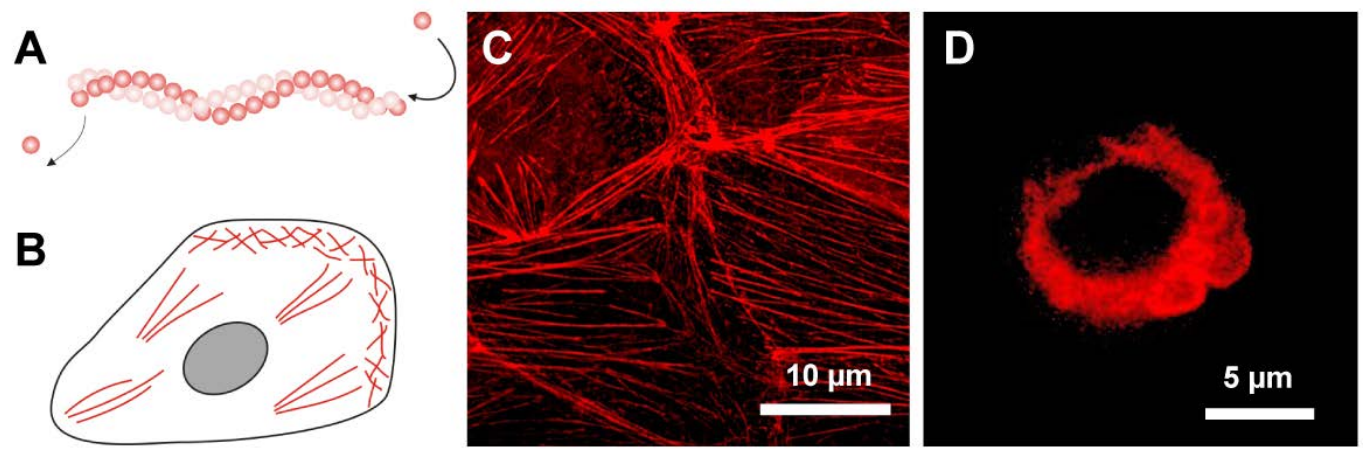

Figure 6: Structure and arrangement of the biopolymer actin. A: Schematic illustration of the ATP driven Actin polymerization. B: Schematic drawing of actin structure within the cell. C: F-actin staining of subconfluent MDCK II cells. D: F-actin staining of an adhering and spreading MDCK II cell.

For cell movment, the deformation of the plasma membrane and its pushing forward is generated by actin polymerization. Different cell types use the following protrutive structures, having a difference in the arrangement of the actin cytoskeleton. 1. Filopodia are expanded by fibroblasts and migrating growth cones. Inside these structures bundelt actin filaments are found. 2. Lamellipodia formed by epithelial cells and fibroblasts, show a cross linked mesh of actin in the plane parallel to the substrate (Figure 6 D). 3. Pseudopodia are visible in amoebae and neutrophils displaying a three-dimensional projection filles with actin-filament-gel [30]. Another very important process involving the 
F-actin and cell motion is the contraction of muscle cells, due to the force generation by the motor protein myosin [60].

Actin filament are semi-flexible structures in contrast to the rigid microtubules exhibiting peristance length in the order of millimeters. Important for the mechanical strength of Factin is the capability of further organization of the filaments as bundling to stress fibers (Figure $6 \mathrm{C}$ ) due to the association of different actin binding proteins like $\alpha$-actinin and filamin [59].

F-actin supports membrane protrusions, the so called microvilli, which are finger-like structures on the apical surface of secretory and absorbing cells. Mircovilli increase the surface and modulate the comunication with the enviroment. One absorptive epithelial cell in the human small intestine is able to develop several thousand microvilli on the apical plasma membrane [30].

\subsubsection{Microtubules}

Microtubules are rigid and hollow cylindrical structures composted of alternating of $\alpha$ - and $\beta$-proteins subunits (tubulin). The polymerization and depolymerization of both biopolymers F-actin and microtubules is a energy depended process, where nucleoside triphospates are hydrolyzed (for actin ATP and for microtubules GTP).
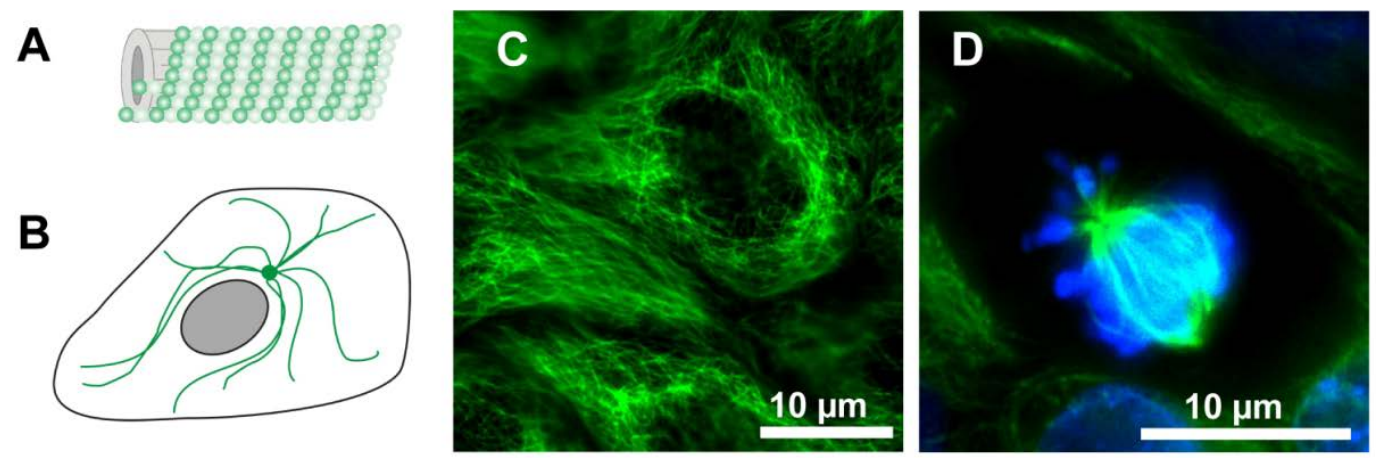

Figure 7: A: Subunit of microtubules is a heterodimer, a linked pair of $\alpha$ - and $\beta$-tubulin. B: Structure and arrangement of microtubules inside a cell. C: Fluorescence micrograph of immustaining of microtubules of confluent MDCK II cells. D: Fluorescence staining of the nucleus (blue) and the microtubules (green) during cell division.

Microtubules provide the structural organization for intracellular transport. There are many microtubule binding proteins like motor proteins such as kinesin (plus-end directed) and dynin (minus-end directed) [61]. It was also shown that microtubules and the associated 
motor proteins act in concert with F-actin and myosin, thereby promoting the tubulation of the plasma membrane [62]. One of the most important structures involving microtubules is the mitotic spindle used during cell division to separate the chromosomes. In almost all animal cells, the MTOC (microtubules-organizing center) or centrosome is near the nucleus and the place where microtubules start to grow (Figure 7 B), in particular from the minus end. Hence the plus pole is reaching and growing to cell periphery.

\subsubsection{Intermediate Filaments}

Intermediate filaments play a structural role and are found in cells which withstand high mechanical stress. The term intermediate filaments results from their diameter $(15 \mathrm{~nm})$, lying between the one of actin (6-8 $\mathrm{nm})$ and microtubules $(25 \mathrm{~nm})$. The genes expressing intermediate filaments are not highly conserved around different cell types (50 different IF genes in humans) leading to different proteins developing intermediate filaments, e.g. keratin intermediate filaments are found in epithelial cells, vinculin intermediate filaments in fibroblasts and endothelial cell, and desmin intermediate filaments in muscle tissue. Intermediate filaments are found in close vicinity of the nuclear membrane[30].
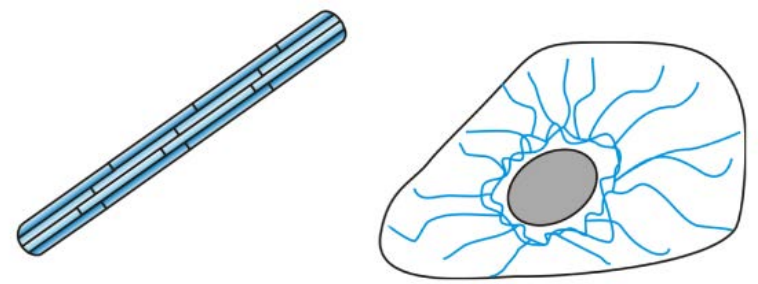

Figure 8: A: Drawing of the structure of an intermediate filament. B: Schematic illustration of the arrangement of intermediate filaments inside a cell.

To form a filament two monomers pack in parallel way building a coiled-coil dimer. Two dimers orientate together generating an anti-parallel tetramer of four polypeptide chains. In the final filament the tetramers are bundled together in a helical array, forming strong ropelike structures, which tolerate stretching and bending [30]. Genetic disorder of intermediate filaments manifests in blistering skin diseases like Epidermolysis Bullosa Simplex [63].Without an appropriate intermediate filament network, cells become fragile and tend to break upon mechanical stress [63]. 


\subsubsection{Mechanical Properties of the Cytoskeleton Components}

Structural differences in the cytoskeleton subunits together with dissimilar polymerization strategies result in the special mechanical properties of the three biopolymers. Intermediate filaments are rope like and easy to bend but hard to break. Microtubules are strong and rigid hollow tubes. Actin filaments are the thinnest filaments. They are hard to stretch but easy to break [30].

Table1: Mechanical characteristics of the three cytoskeleton building filaments [1].

\begin{tabular}{ccccc}
\hline & $d[\mathrm{~nm}]$ & $\kappa\left[\mathrm{N} \mathrm{m}^{2}\right]$ & $E[\mathrm{GPa}]$ & $L_{p}$ \\
\hline actin & $6-8$ & $7 \cdot 10^{-26}$ & $1.3-2.5$ & $15 \mu \mathrm{m}$ \\
microtubules & 25 & $2.6 \cdot 10^{-23}$ & 1.9 & $6 \mathrm{~mm}$ \\
intermediate filaments & 10 & $4-12 \cdot 10^{-27}$ & $1-5$ & $1-3 \mu \mathrm{m}$ \\
\hline
\end{tabular}

$d=$ diameter; $\kappa=$ bending stiffness; $E$ = Young's modulus; $L_{p}=$ persistence length

Janmey et al. performed important mechanical measurements of the three components of the cytoskeleton. In their work, the authors show the stress-strain characteristics of actin, vimentin, fibrin and tubulin [64]. The stress/strain behavior of a F-actin network compared to the other polymers shows that F-actin resists to large deformation until a critical value (20\% strain). These properties are responsible for developing the cellular cortex, since the network can "fluidize” under large shear stress to allow cell movement. This brings F-actin into focus regarding the cytoskeleton's contribution to the mechanical properties of the cellular plasma membrane.

For this reason, the change in the F-actin organization and the occurrence of its binding partner's are suitable mechanisms to manipulate cellular mechanics. Investigating the cellular response to rearrangements of the cortical cytoskeleton structure underlying the plasma membrane will provide more inside into the mechanics of the plasma membrane and how the adhesion of the cytoskeleton to the membrane contributes to it. 


\subsection{Membrane Mechanics}

When studying plasma membrane mechanics, the fundamental mechanical properties of the lipid bilayer must be considered. The mechanical properties rely on the chemical nature of the membrane components and the forces acting between them. The elastic behavior of the membrane is governed by the elastic stretching, bending rigidity and in-plane tension (Figure 9).
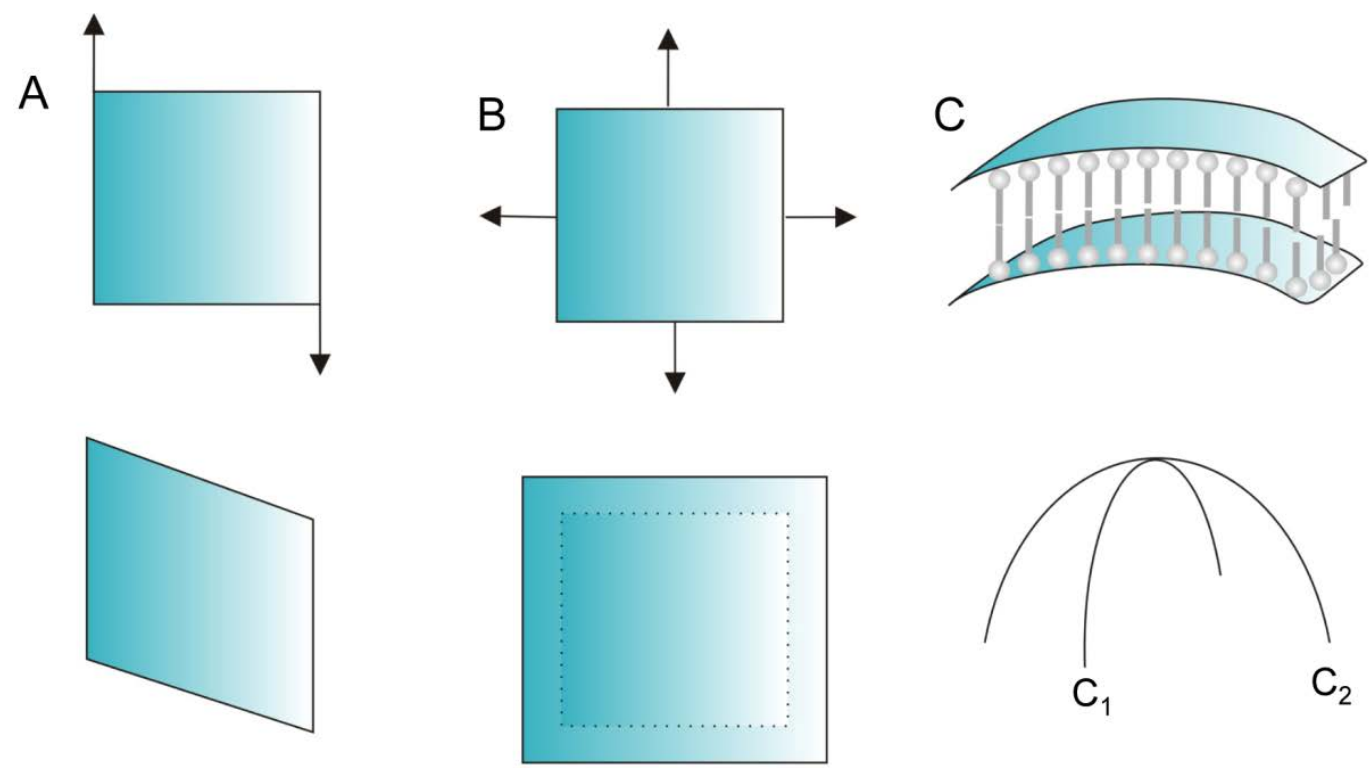

Figure 9: Illustration of membrane deformation. A: In-plane shearing. B: In-plane stretching. C: Bending results in an out of plane deformation described by its two curvatures $C_{1}$ and $C_{2}$. Modified from [65].

A theoretical description of membrane deformation derived by Helfrich and Canham in the 1970’s [66] [67].

\subsubsection{Shearing and Stretching}

Shearing is the deformation due to the action of parallel forces in opposite direction, without changing the area (Figure 9 A). A pure fluid lipid bilayer shows no resistance to shear deformation, because the lipids from the outer monolayer flow past the others lipids from the inner monolayer. But regarding the cellular plasma membrane, the subsurface cytoskeleton, which is anchored to the membrane, introduces shear rigidity to the bilayer [32]. The energy per unit area $\left(E_{\text {shear }}\right)$ is according to Hook's law: 
$E_{\text {shear }}=\frac{1}{2} \mu\left(\left(\frac{L_{0}+\Delta L}{L_{0}}\right)^{2}+\left(\frac{L_{0}+\Delta L}{L_{0}}\right)^{-2}-2\right)$

$L_{0}$ is the initial length of the membrane before deformation, and $\mu$ the shear modulus $(\mu=$ 0 , for fluid lipid bilayer). $\mu$ was measured to be $6 \cdot 10^{-3} \mathrm{mN} \mathrm{m}^{-1}$ for red blood cells [68] [65].

Stretching of a material from a surface $A_{0}$ (surface before expansion) to $A$ costs energy (Figure 9 B). The energy change can be expressed as followed [69]:

$E_{\text {strech }}=\frac{1}{2} K_{A}\left(\frac{A-A_{0}}{A_{0}}\right)^{2}$,

with $K_{A}$ as area compressibility modulus. The change in membrane area can be measured by different techniques, e.g. micropipette aspiration. A typical value for $K_{A}$ is around 0.25 $\mathrm{N} \mathrm{m}^{-1}$ [70]. Membranes are poorly stretchable. This is an effect due to the packing of the lipid molecules in the bilayer. Slight additional separation of the lipid heads ( 2\%) leads to water entry between the acyl chains and destabilize the structure basted on the hydrophobic interaction. Bilayer can only expand 2-4 \%, before they rupture [71].

\subsubsection{Bending}

Bending is a result of deforming the membrane out of the plane. The corresponding areal energy density is:

$E_{\text {bend }}=\frac{1}{2} \kappa\left(C_{1}+C_{2}-C_{0}\right)^{2}+\kappa_{G} C_{1} C_{2} \quad$,

$\kappa$ is the bending rigidity modulus and $\kappa_{G}$ the Gaussian curvature modulus. $C_{1}$ and $C_{2}$ represents the principle membrane curvatures $\left(C_{1}=R_{1}^{-1}\right.$ and $\left.C_{2}=R_{2}^{-1}\right)$ and $C_{0}$ the spontaneous curvature. The bending rigidity $\kappa$ is expressed in units of $\mathrm{J}$ or $\mathrm{k}_{\mathrm{B}} \mathrm{T}$. Taken into numbers $\kappa$ of fluid lipid bilayers is typically in the range of $10^{-19} \mathrm{~J}$ ( $\sim 25 \mathrm{k}_{\mathrm{B}} \mathrm{T}$ ), depending on the lipid composition [70].

A second approach to figure out the bending rigidity is to consider that bending involves both the compression and expansion of the two layer of the membrane. $\kappa$ can be related to the area compressibility modulus $K_{A}$ with and the thickness of the bilayer $h$ [69]: 
$\kappa=\frac{1}{c} K_{A} h^{2}$

Where $c=48$ for two uncoupled elastic sheets and $c=12$ for a single elastic sheet [69].

Since bending leads to uniaxial extension (or compression) out of the membrane's plane (Figure $9 \mathrm{C}$ ), the three-dimensional Young's modulus $E$ can be expressed using the twodimensional stretching modulus $K_{A}$ defined in Eq. (2) [69]:

$K_{A}=E h$

\subsubsection{Tension}

The lateral stress or tension exerted on a membrane by area dilation is per definition the derivative of energy with respect to area [69]:

$T=\frac{\partial E_{\text {stretch }}}{\partial A}=K_{A} \frac{A-A_{0}}{A_{0}}$

From the observation and measurement of membrane undulation the following expression is considered [27]:

$\widetilde{K}_{A}=\frac{K_{A}}{\frac{1+K_{A} k_{B} T}{8 \pi \kappa T}}$

gives the apparent area compressibility modulus $\tilde{K}_{A}$. Equation 7 describes the observation that the effective in-plane area of a membrane is reduced by thermal fluctuations. As a consequence an applied tension to a membrane flattens out these fluctuations first before increasing the true area of the bilayer [27].

At small tensions the apparent area compressibility modulus can be approximated by [27]

$\widetilde{K}_{A} \sim \frac{8 \pi \kappa T}{k_{B} T}$

The overall plasma membrane tension is a result of the contributions from the intrinsic surface tension of the lipid bilayer, the molecular connection of the plasma membrane to the underlying actin-cytoskeleton [10], and tension generating through cell-cell contacts (for adhering and confluent cells). This implies that by regarding the cellular membrane an 
additional component accounts for the pre-stress generated by proteins in the plasma membrane.

$T=T_{0}+K_{A} \frac{A-A_{0}}{A_{0}}$

Membrane theory with an additional pre-stress term is more suitable to describe cellular elasticity of fluid-like cells. Therefore we additionally insert into Equation (6) a term describing the pre-tension $T_{0}$ of the membrane. 


\section{Theory}

\subsection{Mechanical Models}

Studies have shown, that many biological processes like growth, migration, differentiation and apoptosis lead to changes in cell shape and structural integrity [72]. Variation in these structural and mechanical properties can promote the breakdown of physiological functions and hence leads to diseases [1]. One prominent example, giving inside into the complex interplay between the function of an organism and cellular mechanics, is the disease malaria. The most dangerous form of malaria is caused by the parasite Plasmodium falciparum leading to a strong molecular and structural alteration of red blood cells. These cells are very flexible and transport oxygen even through the smallest vessel by mechanical deformation. The parasite causes a stiffening of the cell membrane [73] and as a result endangers red blood cells function. The connection between changes in cellular mechanics and diseases like cancer are well known and up to this day under investigation [74] [1].

Table 2: Techniques used to study cellular mechanics [72]

\begin{tabular}{|c|c|c|}
\hline Technique & Subject & Reference \\
\hline Magnetic particle method & $\begin{array}{l}\text { Frontal bone of chick } \\
\text { embryo }\end{array}$ & $\begin{array}{l}\text { Crick and Hughes } 1950 \\
\text { [75] }\end{array}$ \\
\hline Micropipette aspiration & $\begin{array}{l}\text { Elastic properties of sea } \\
\text { urchin eggs }\end{array}$ & $\begin{array}{l}\text { Mitchison and Swann } \\
1954 \text { [76] }\end{array}$ \\
\hline Micropipette aspiration & $\begin{array}{l}\text { Mechanical properties of } \\
\text { red blood cell membrane }\end{array}$ & $\begin{array}{l}\text { Rand and Burton } 1964 \\
\text { [77] }\end{array}$ \\
\hline Cell poker & Mouse 3T3 fibroblasts & Petersen et al. 1982 [78] \\
\hline Particle tracking & PTK-2 cells & Geerts et al. 1987 [79] \\
\hline $\begin{array}{l}\text { Magnetic twisting } \\
\text { cytometry (MTC) }\end{array}$ & $\begin{array}{l}\text { Capillary endothelial } \\
\text { cells }\end{array}$ & Wang et al. 1993 [80] \\
\hline $\begin{array}{l}\text { Oscillatory magnetic } \\
\text { twisting cytometry }\end{array}$ & $\begin{array}{l}\text { human airway smooth } \\
\text { muscle cells }\end{array}$ & $\begin{array}{l}\text { Maksym et al. } 2000 \\
\text { [81] }\end{array}$ \\
\hline $\begin{array}{l}\text { Atomic force microscopy } \\
\text { (AFM) }\end{array}$ & $\begin{array}{l}\text { apical surface of MDCK } \\
\text { monolayers }\end{array}$ & $\begin{array}{l}\text { Hoh and } \\
\text { Schoenenberger } 1994 \\
\text { [82] }\end{array}$ \\
\hline Microplate manipulation & $\begin{array}{l}\text { Spreading of chick } \\
\text { fibroblasts }\end{array}$ & $\begin{array}{l}\text { Thoumine and Ott, } \\
1997 \text { [83] }\end{array}$ \\
\hline Optical tweezers & human erythrocytes & Henon et al, 1999 [84] \\
\hline Magnetic bead pulling & human red blood cells & Mills et al. 2004 [85] \\
\hline
\end{tabular}


The main challenge when studying cellular mechanics is to use an appropriate mechanical model that fits experimental data without suffering from mismatching assumptions. Techniques used to investigate mechanical properties of cells are summarized in Table 2.

In the following sections two different mechanical models are presented. The first one, the Hertzian approach describes the cell as solid elastic body. The second one is a cortical shell - liquid core model and characterizes the cell as viscoelastic liquid droplet. The used mechanical technique in this thesis is the atomic force microscope. Hence, the description of the mechanical models is referred to AFM indentation experiments using a conical probe.

\subsubsection{Hertzian Mechanics}

Since the earliest years of the AFM, the "Hertz-model" is used to examine the mechanical properties of cells [86] [87] [88]. Originally this model was established by Heinrich Hertz in 1881 for the observation of two elastic bodies in contact [89]. By using various assumptions (described below) to this day this model is used to extract the Young's modulus of cells. The Young's modulus (E-modulus) is a material property and has been determined for many materials as well as soft samples as cells (Figure 10) [90] [91].

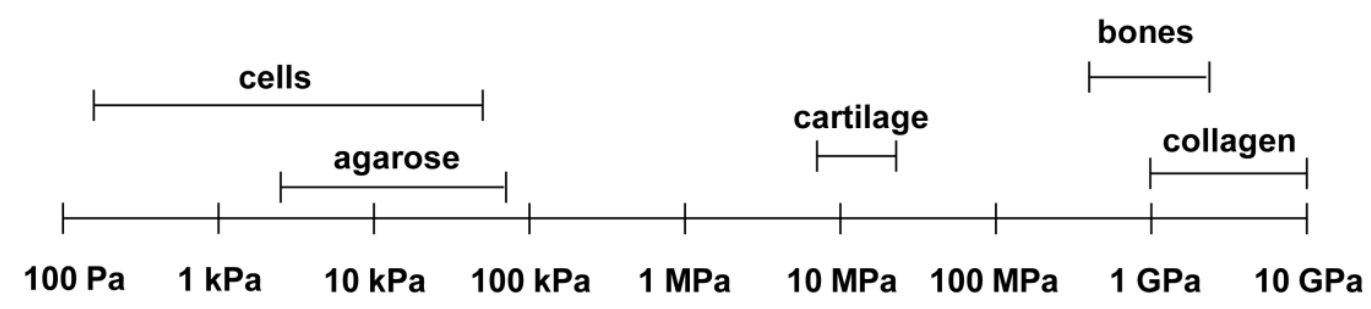

Figure 10: Illustration of Young’s modules of different materials from soft to hard. Modified from [92].

The interpretation of the results from force-indentation-relationships require exact solution for indenter geometries, which can be found by Sneddon (1965) [93]. 


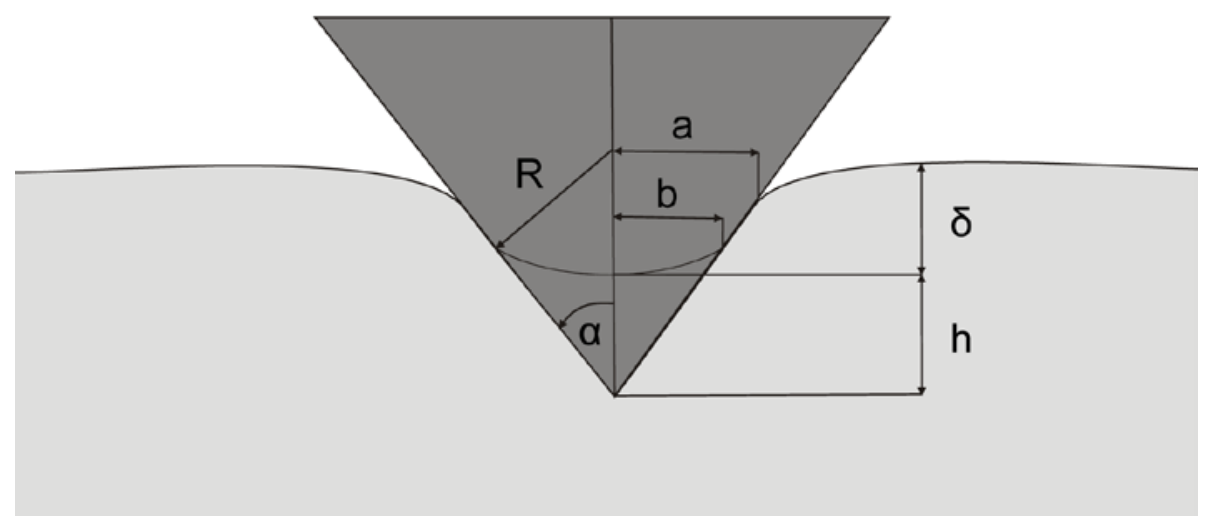

Figure 11: Both conical and pyramidal tips are shown, together with a blunt tip. $a$ is the contact radius. $\alpha$ is the half-opening angle of cone or pyramid. $b$ is the radius at which the tapered sides transition into a spherical tip of radius $R$. Transition from blunt tip to sharp tip, when $R=b=0$, modified from [94].

A general formulation for the force-displacement-relationship is [94] [95] [96]:

$$
F=g \frac{E}{(1-v)} \delta^{n} \quad(1 \leq n \geq 2)
$$

With $F$ the applied force, $E$ the Young's modulus, $v$ Poisson's ratio ( 0.5 for incompressible sample), $\delta$ the indentation depth and $g$ and $n$ as parameters describing the tip geometry.

For a tapered indenter the relationship is according to Figure 11:

$$
F=\frac{2 E}{(1-v)^{2}}\left\{a \delta-m \frac{a^{2}}{\tan \alpha}\left[\frac{\pi}{2}-\arcsin \frac{b}{a}\right]-\frac{a^{3}}{3 R}+\left(a^{2}-b^{2}\right)^{\frac{1}{2}}\left[m \frac{b}{\tan \alpha}+\frac{a^{2}-b^{2}}{3 R}\right]\right\}
$$

Inserting $m=1 / 2$, gives the Equation for a conical indenter; $m=2^{1 / 2} / \pi$ turns the indenter to a pyramid.

The contact radius is related to the indentation depth by:

$$
\begin{aligned}
& \delta+\frac{a}{R}\left[\left(a^{2}-b^{2}\right)^{\frac{1}{2}}-a\right]-n \frac{a}{\tan \alpha}\left[\frac{\pi}{2}-\arcsin \frac{b}{a}\right]=0 ; \\
& n_{\text {cone }}=1 n_{\text {pyramid }}=2^{3 / 2} / \pi
\end{aligned}
$$

By considering a conical indenter, Equations (14) simplifies to:

$a=\frac{2 \delta \tan \alpha}{\pi}$,

Inserting Equation (12) in (10) yields the "Hertz-model” for a conical indenter:

$F=\frac{E}{(1-v)^{2}} \frac{2 \tan \alpha}{\pi} \delta^{2}$ 


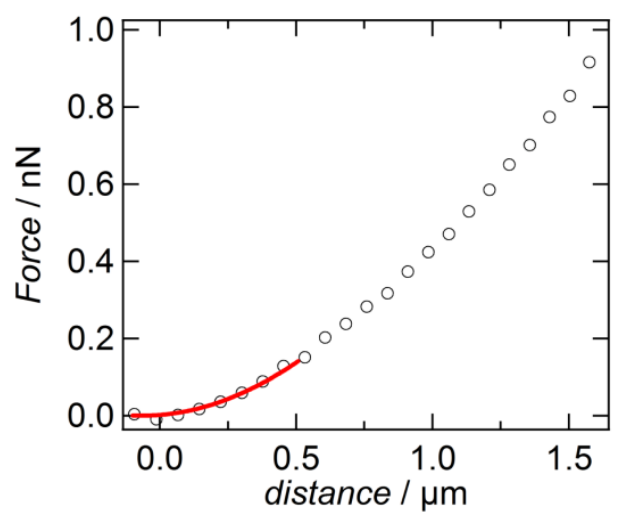

Figure 12: Force distance trace curve recorded on a MDCK II cell (dots). In red, the Hertz-model is fitted to experimental data.

The original sample properties by applying the "Hertz-model" are considered to be thick and homogenous, isotropic, and linear elastic with a smooth surface. Also for the interpretation of cellular mechanics, the "Hertz-model" is based on these assumptions. Using the Herzian approach to calculate the Young's modulus of a sample should be done with respect to the basic assumptions. Domke and Radmacher report that the Young'smodulus heavily relies on sample thickness [97]. Therefore, we fit the "Hertz-model" to a maximal indentation depth of $0.5 \mu \mathrm{m}$. 


\subsubsection{Liquid Droplet-Model}

This model was first established to describe the viscoelastic behavior of neutrophils and leucocytes in micropipette aspiration experiments [98]. These cells behave like liquid droplets when suspended and deform continuously inside a micropipette. To depict this observation the cell interior is assumed to be a homogeneous Newtonian viscous liquid and the cell cortex as viscose layer with static tension without bending resistance. Additionally, the velocity field is assumed to be continuous at the interface between the cortical layer and the liquid core [72].

Robert Hochmuth states that although the cell is shaped as a sphere when detached from the substrate, this not means that the cell should be modeled as liquid drop with constant surface tension. He point out further: "what the observation does mean is that a "cortical tension” exists at the surface of the cell and this cortical tension is, in all likelihood responsible for pulling the cell into this spherical shape“ [99].

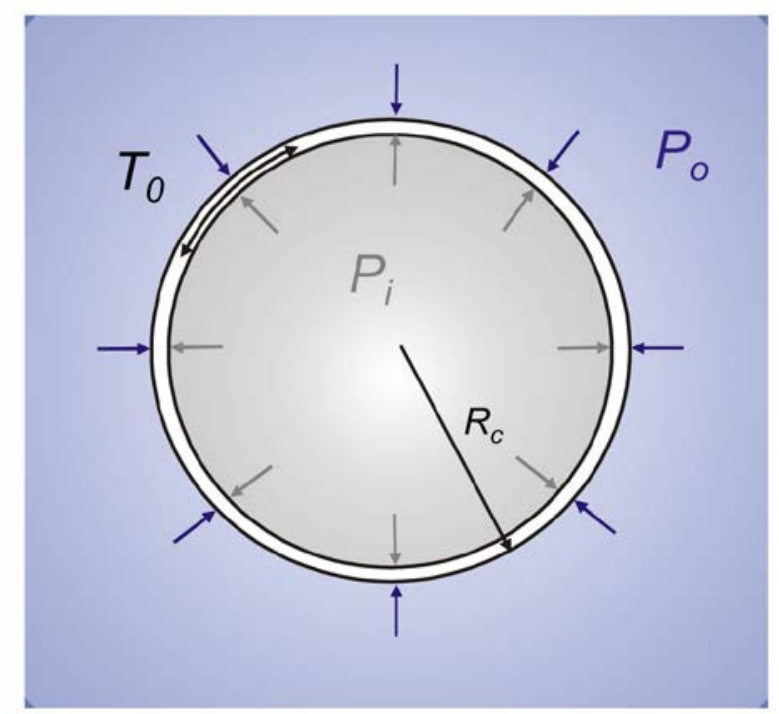

Figure 13: Force balance in a sphere with constant surface tension.

The main advantage of this model is the possibility to measure the cortical tension of spherical cells. The Laplace Law predicts the force balance for the sphere [99]:

$$
\begin{aligned}
& 2 \pi R_{C} T_{0}=\left(P_{i}-P_{o}\right) \pi R_{c}^{2} \\
& \left(P_{i}-P_{o}\right)=\frac{2 T_{0}}{R_{c}}
\end{aligned}
$$


With $\Delta P=P_{i}-P_{0}$ the pressure difference across the interface; $T_{0}$ surface tension and $R_{c}$ the radius of the cell. Equation (15) shows that $T_{0}$ cause the interior of the cell to be under pressure with respect to the pressure outside. For the most cell lines it is more appropriate to model the cell with a cortical surface tension rather than assuming a homogeneous elastic body characterized by a single Young's modulus, since the membrane underlying cortex exerts tension to the membrane. Typical values found for cortical tension measured with micropipette aspiration, are in between of $0.02-0.3 \mathrm{mN} \mathrm{m}^{-1}$ [72].

Although this model was invented to describe the continuous deformation of a spherical cell into a micropipette, the liquid droplet theory can be extended to other experimental conditions like the AFM technique and either be applied to spherical cells in suspension (Rosenbluth et al. [24]) or to cells adhered to a substrate (Sen et al. [25]). The principle idea that the cell membrane and the associated cytoskeleton can be modeled as a cortical layer with negligible viscosity [98] and with a constant surface tension remains valid. 


\subsection{Model Extension}

\subsubsection{Modeling Spherical Cells}

Lomakina and coworkers extended the liquid droplet model for the indentation of a cell with a bead [100] and Rosenbluth et al. processed the model for the indentation with an AFM tip with pyramid geometry [24]. To describe the experiments done in this thesis, the geometry of the indenter is changed from a pyramid to a cone to account for the right geometry of the indenter.

Generally, in the present work, it is assumed that MDCK II cells behave as a liquid droplet with an isotropic in-plane tension. The typical non-linearity observed in force indentation experiments performed on epithelial cells (Figure 15) suggests that membrane theory with an additional pre-stress term is more suitable to describe cellular elasticity of fluid-like cells than assuming a homogeneous elastic body characterized by a single Young's modulus.

Mechanics is therefore governed predominately by area dilatation of membrane and cortex rather than bending or bulk elasticity. Since the membrane of a cell is very thin ( $\sim$-5 $\mathrm{nm})$ bending of the shell is neglected [101]. In this approach the cell is in static equilibrium with the indenter.

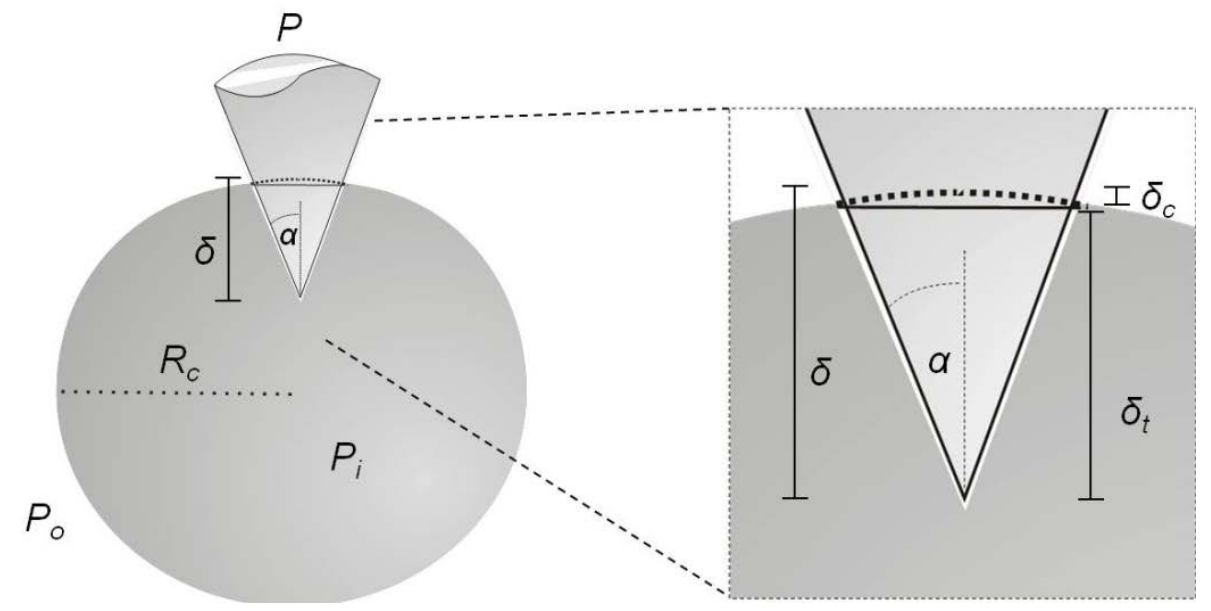

Figure 14: Illustration of a conically shaped probe indenting a spherical, non-adhering cell. $P_{i}, P_{o}$ and $P$ are the pressure of the cell interior, fluid surrounding the cell, and tip. The opening angle of the cone is $\alpha$. $R_{c}$ is the radius of the cell. 
The force experienced by a conical indenter amounts to:

$F=2 \pi T\left(\delta_{t} \tan \alpha\right)^{2}\left(\frac{1}{R_{c}}+\frac{1}{\tan \alpha}\right)$

with

$\delta=\delta_{t}+R_{c}-\sqrt{R_{c}^{2}-\left(\delta_{t} \tan \alpha\right)^{2}}$

$T$ denotes the isotropic lateral tension and $\delta_{t}$ the height of the cone immersed in the cell with an opening angle $\alpha$. $\delta$ denotes the indentation depth and $\delta_{c}$ is the distance of the sphere prior to indentation to the base of the cone (Figure 14).

Notably, $T$ is also a function of the indentation depth since it depends on the area dilatation (vide infra). The model assumes a constant isotropic tension $T_{0}$ extended by an area dilatation term that accounts for in-plane stretching during indentation, according to equation (9).

$\widetilde{K}_{A}=K_{A} \frac{A_{0}}{A_{0}+A_{e x}}$

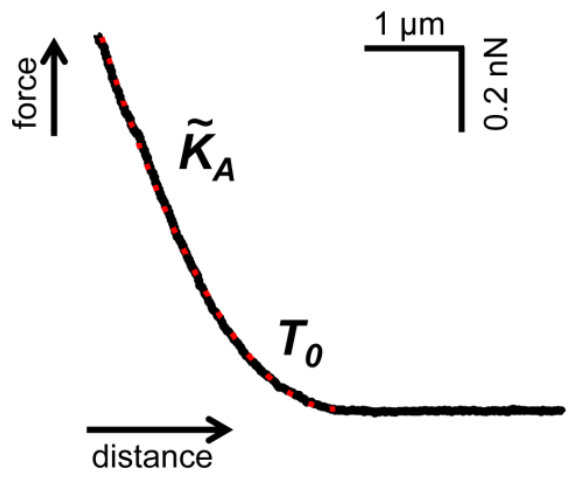

Figure 15: Representative force-distance trace curve (black line) is used for determine the area compressibility modulus $\tilde{K}_{A}$ and tension $T_{0}$ using a modified liquid droplet model for indentation experiments fitted to the experimental data (red dots).

The pre-stress $T_{0}$ governs the response to indentation at low penetration depth, while nonlinear in-plane stretching is captured by the apparent area compressibility modulus $\tilde{K}_{A}$ of the shell at larger stains. $\Delta A$ denotes the change in surface area and $A_{0}$ is the initial area of the cell. $\tilde{K}_{A}$ is a measure of excess membrane surface since it becomes smaller if excess membrane area stored in reservoirs such as caveolae and protrusions exists, while it assumes values of the order of 0.1 to $1 \mathrm{~N} / \mathrm{m}$ if reservoirs are absent. This upper limit is set 
by the inextensibility of the plasma membrane generating an area compressibility modulus of 0.1 to $1 \mathrm{~N} / \mathrm{m}$ depending on the composition of the bilayer.

\subsubsection{Modeling Adherent Cells}

According to the liquid-drop model the adherent cell is also considered as an isotropic shell that produces a restoring force in response to indentation with a conical indenter originating from two sources, linear elasticity due to area dilatation and pre-stress (constant tension). Pre-stress is mainly generated by strong adhesion to the substrate and interaction of the plasma membrane with the cytoskeleton. Bending, however, plays a minor role and is therefore neglected.

The cell shape is obtained from the radius of the contact zone $R_{i}$ and the wetting angle $\phi$ as illustrated in Figure 16 assuming constant curvature and constant volume [25]. The model assumes a constant isotropic tension $T_{0}$ (Equation 9) extended by an area dilatation term that accounts for in-plane stretching during indentation (Equation 19), as described for the spherical cell.

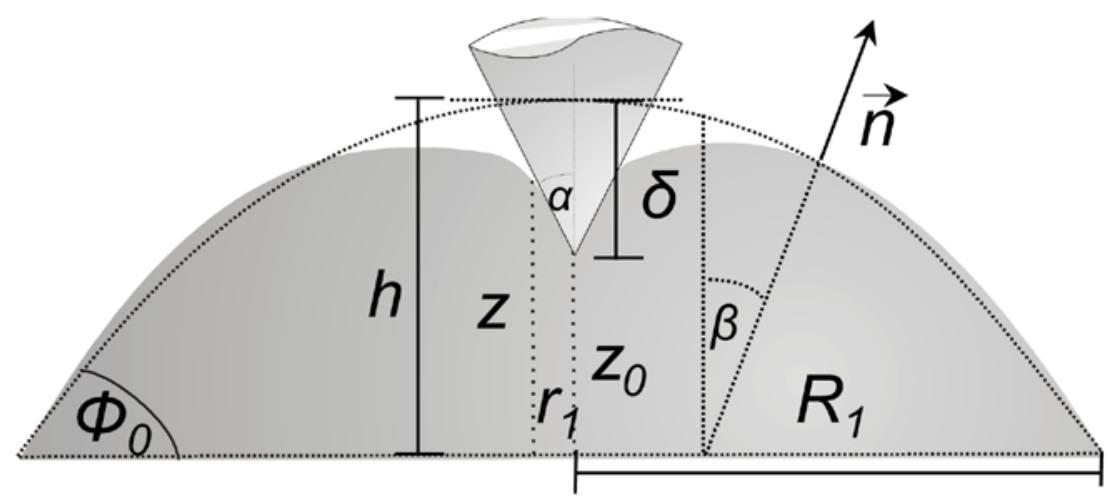

Figure 16: The cell is described by a spherical cap. $R_{1}$ is the base radius, $r_{1}$ the radial position beyond which the membrane is not in contact with the tip. $\phi_{0}$ the contact angle between cell and substrate, $\delta$ the indentation depth and h the cell height before indentation.

Static equilibrium can be expressed by the Laplace Law (Equation 15, 21), which describes the pressure difference between cell interior and exterior as a function of surface tension $T$ and mean curvature $H=R_{m}{ }^{-1}+R_{\phi}^{-1}$.

$\Delta P=2 T H$

The force equilibrium at the base is defined by geometrical parameters of the spherical cap giving a linear dependency for $F$ :

$\pi R_{1}^{2} T 2 H=F+2 \pi R_{1} T \sin \phi$ 
$F=2 T\left(\pi R_{1}^{2} H-\pi R_{1} \sin \phi\right)$

Since the volume of the cell is assumed to be constant during indentation, the volume can be computed from the shape (shape function $u$, Equation 24) and be set equal to the volume before indentation (Equation 23).

$V=\int_{r_{1}}^{R_{2}} \pi r^{2} \frac{u(r)}{\sqrt{1-u(r)^{2}}} d r-\frac{\pi r_{1}^{3}}{3 \tan \phi}$

$u=A r+\frac{B}{r}$

with

$A=\frac{R_{1} \sin \phi+r_{1} \sin \alpha}{R_{1}^{2}-r_{1}^{2}}$

and

$B=-A r_{1}^{2}-r_{1} \sin \alpha$

$V_{1}=\left(R_{0}-h\right) \frac{\pi h^{2}}{3}=V$

$h=R_{0}-\sqrt{R_{0}^{2}-h}$

with $R_{0}$ the cell radius before adhesion and $h$ the cells height before indentation. The two linear Equations (23) and (24) are solved for $r_{1}$ and $\phi$ and with this the z-position can be calculated to compute the deformed shape of the membrane:

$d z=d r \tan \beta$

where $\beta$ is the angle at which the normal at any point on the surface meets the vertical axis [25].

Finally we can calculate the indentation depth $\delta$ :

$$
\begin{aligned}
& z_{0}=z\left(r_{1}\right)-r_{1} \tan \alpha \\
& \delta=h-z_{0}
\end{aligned}
$$

This enables us to extract from force indentation experiments the membrane tension $T_{0}$ and apparent area compressibility modulus $\tilde{K}_{A}[102]$.

In section 5.8.3 mechanical values obtained from the liquid droplet model for spherical cells are compared to values from the liquid droplet model for adherent cells. 


\subsubsection{Geometrical Description of Adherent MDCK II Cells}

The cell shape needs to be considered carefully as it bears important input parameters for the mechanical approach according to Sen et al. [25]. It is assumed that the cell-cell contacts connected to the contractile actin ring serve as tension-generating boundaries justified by AFM topography images of confluent untreated cells (Figure 29) that display cell-cell contacts as slightly elevated and more rigid ring-like structures around each cell body.

Typically, a distance between the apexes of the cell to the cell-cell boundaries of about 1 $\mu \mathrm{m}$ was measured. The relevant topographical parameters such as radius of the apex and contact angle are determined by AFM imaging. From data analysis we infer a radius of the apex of $R_{0}=35 \mu \mathrm{m}$ and a contact angle of $\phi=20^{\circ}$. This approach leads to the modeling of a spherical cap representing only the apical part of the confluent cells (Figure 17 and Figure 35).

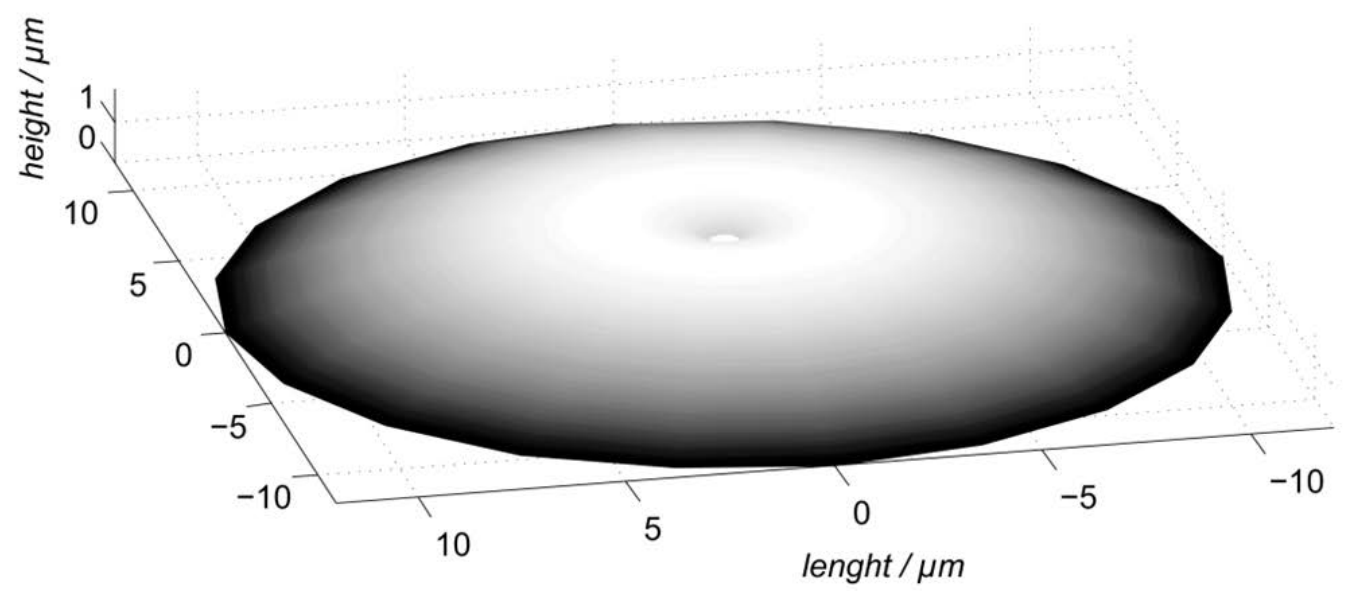

Figure 17: Simulation of a spherical cap representing the apex of a confluent MDCK II cell.

Experimental data (force distance curve) obtained from indentation of MDCK II cells with an AFM-tip are almost identical with simulated force distance curves. This confirms that the modeling of the apex is correct and describes the experimental data (Figure 18). 


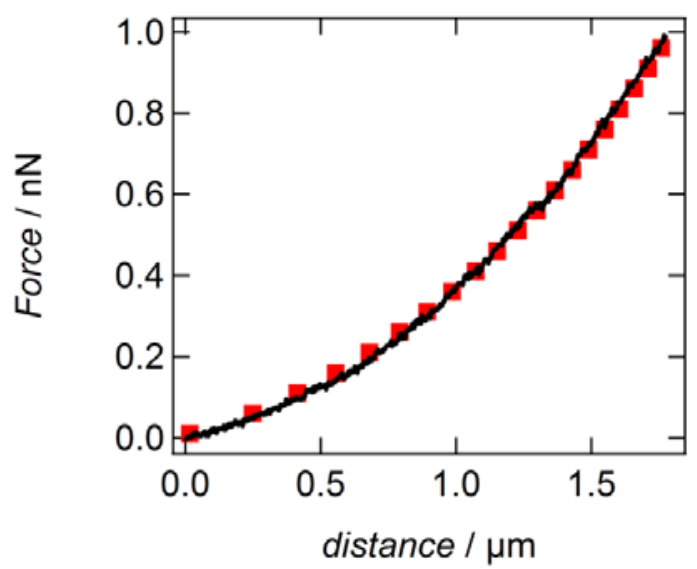

Figure 18: Force distance curve recorded on a confluent MDCK II cell (black). Red squares represent a simulated force distance curve for a modeled apex with $R_{0}=35 \mu \mathrm{m}$ and a contact angle of $\phi=20^{\circ}$. Mechanical parameters are set to values of: pre-tension $T_{0}=0.3 \mathrm{mN} \mathrm{m}^{-1}$ and $\tilde{K}_{\mathrm{A}}=0.08 \mathrm{~N} \mathrm{~m}^{-1}$. 


\subsubsection{Simulation}

In the following section typical force distance curves are computed according to the liquid droplet model for different tension and area compressibility modules. At low forces the tension $T_{0}$ dominates the response of the cortex while at larger forces area dilatation sets in giving rise to a non-linear approximately cubic dependence of force on indentation depth [102]. A decrease in the apparent area compressibility modulus leads to pronounced changes in the indentation depth and computed cell shape, while alteration in pre-tension values show only small changes in the computed cell shape and force distance curves (Figure 19).
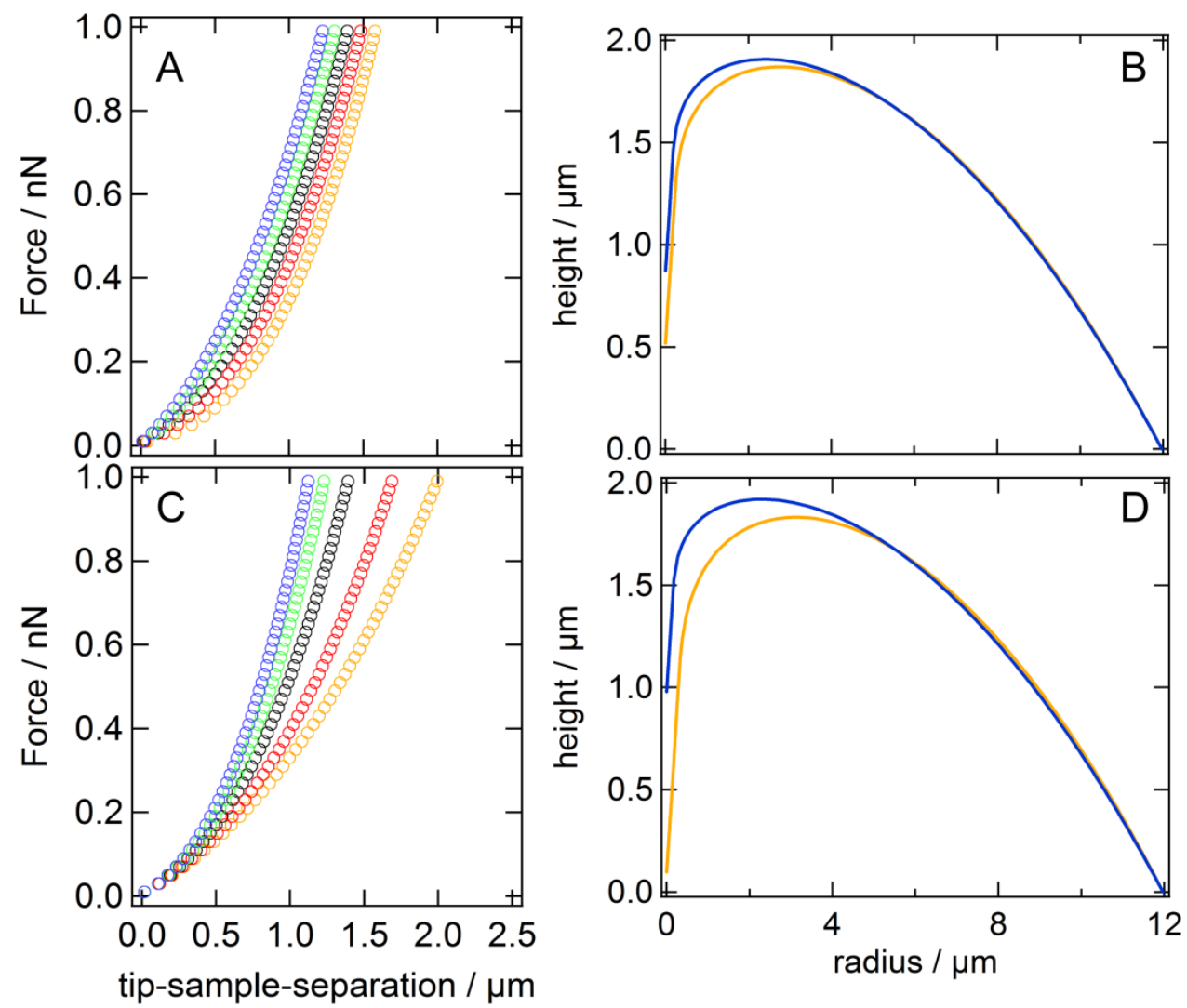

Figure 19: Typical force indentation curves according to the liquid droplet model. A: Simulated force curves assuming an area compressibility of $\tilde{K}_{A}=0.2 \mathrm{~N} \mathrm{~m}^{-1}$ and a pre-tension $T_{0}(\bullet) 0.1 \mathrm{mN} \mathrm{m}^{-1},(\bullet) 0.2 \mathrm{mN} \mathrm{m}^{-1}$, $(\bullet) 0.3 \mathrm{mN} \mathrm{m}^{-1},(\bullet) 0.4 \mathrm{mN} \mathrm{m}^{-1},(\bullet) 0.5 \mathrm{mN} \mathrm{m}^{-1}$. B: Contour of an adherent cell represented by a spherical cap subject to indentation using a conical indenter and a maximal force of $1 \mathrm{nN} . T_{0}$ and $\tilde{K}_{A}$ are set to $0.3 \mathrm{mN}$ $\mathrm{m}^{-1}$ and $(\bullet) 0.05 \mathrm{~N} \mathrm{~m}^{-1},(\bullet) 0.4 \mathrm{~N} \mathrm{~m}^{-1}$. C: Variation of simulated force curves assuming a pre-tension of $T_{0}=$ $0.3 \mathrm{mN} \mathrm{m}^{-1}$ and area compressibility $\tilde{K}_{\mathrm{A}}(\bullet) 0.05 \mathrm{~N} \mathrm{~m}^{-1},(\bullet) 0.1 \mathrm{~N} \mathrm{~m}^{-1},(\bullet) 0.2 \mathrm{~N} \mathrm{~m}^{-1}$, (•) $0.3 \mathrm{~N} \mathrm{~m}^{-1}$, (•) 0.4 $\mathrm{N} \mathrm{m}^{-1}$.D: Contour of an adherent cell represented by a spherical cap subject to indentation using a conical indenter and a maximal force of $1 \mathrm{nN}$. $\tilde{K}_{A}$ and $T_{0}$ are set to $0.2 \mathrm{~N} \mathrm{~m}^{-1}$ and $(\bullet) 0.1 \mathrm{mN} \mathrm{m}^{-1}$, (•) $0.5 \mathrm{mN} \mathrm{m}^{-1}$. A 
initial cell radius $R_{0}=35 \mu \mathrm{m}$ (note that $R_{1}=R_{0} \sin (\phi)$ ), a wetting angle prior to indentation $\phi=20^{\circ}$, a half opening angle of the conical indenter of $\alpha=17.5^{\circ}$ are used for computation.

The adjustment of the cellular shape by changing either the wetting angle prior to indentation $\phi$ or initial cell radius $R_{0}$ yield mainly detectable changes in the area compressibility modulus rather than pre-tension.
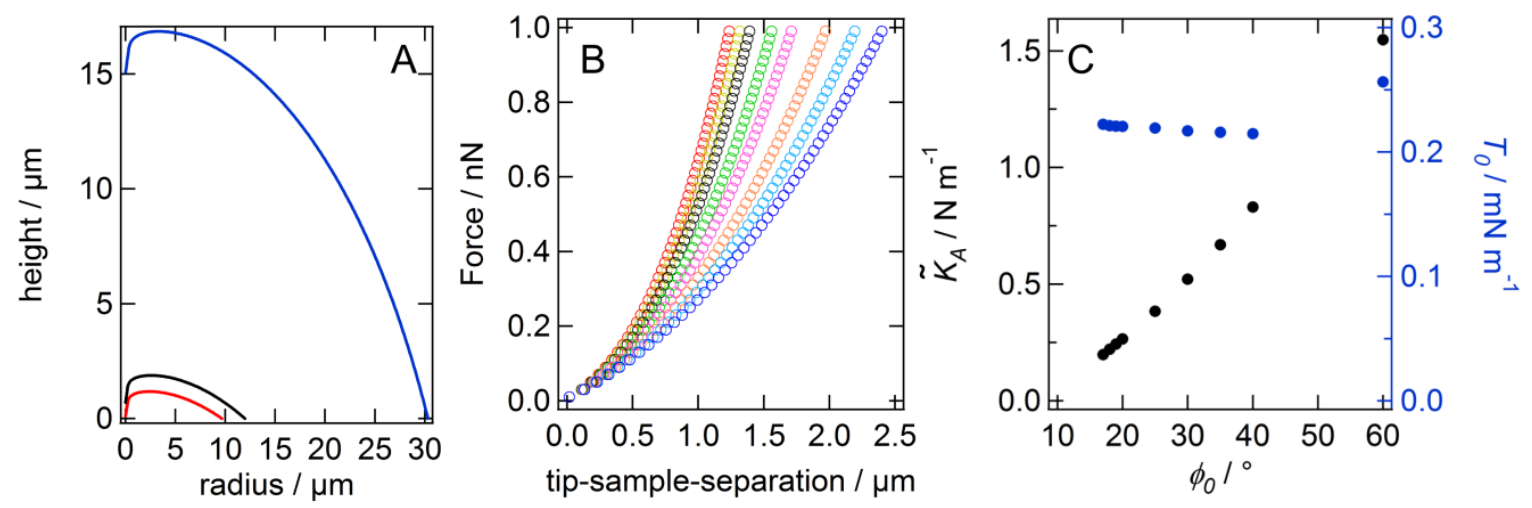

Figure 20: Influence of cellular shape (wetting angle) on mechanical parameter. A: Contour of an adherent cell represented by a spherical cap subject to indentation using a conical indenter and a maximal force of $1 \mathrm{nN}$. A initial cell radius $R_{0}=35 \mu \mathrm{m}$ (note that $R_{1}=R_{0} \sin (\phi)$ ), a wetting angle prior to indentation $\phi=(-)$ $16^{\circ}$, (-) $20^{\circ}$, (-) $60^{\circ}$, a half opening angle of the conical indenter of $\alpha=17.5^{\circ}$ are used for computation. B: Simulated force curves assuming a wetting angle prior to indentation $\phi=(\bullet) 16^{\circ},(\bullet) 18^{\circ},(\bullet) 20^{\circ},(\bullet) 25$ $\circ,(\bullet) 30^{\circ},(\bullet) 40^{\circ},(\bullet) 50^{\circ},(\bullet) 60^{\circ}$. C: $\tilde{K}_{A}$ increases with growing wetting angle, while $T_{0}$ stays almost constant.
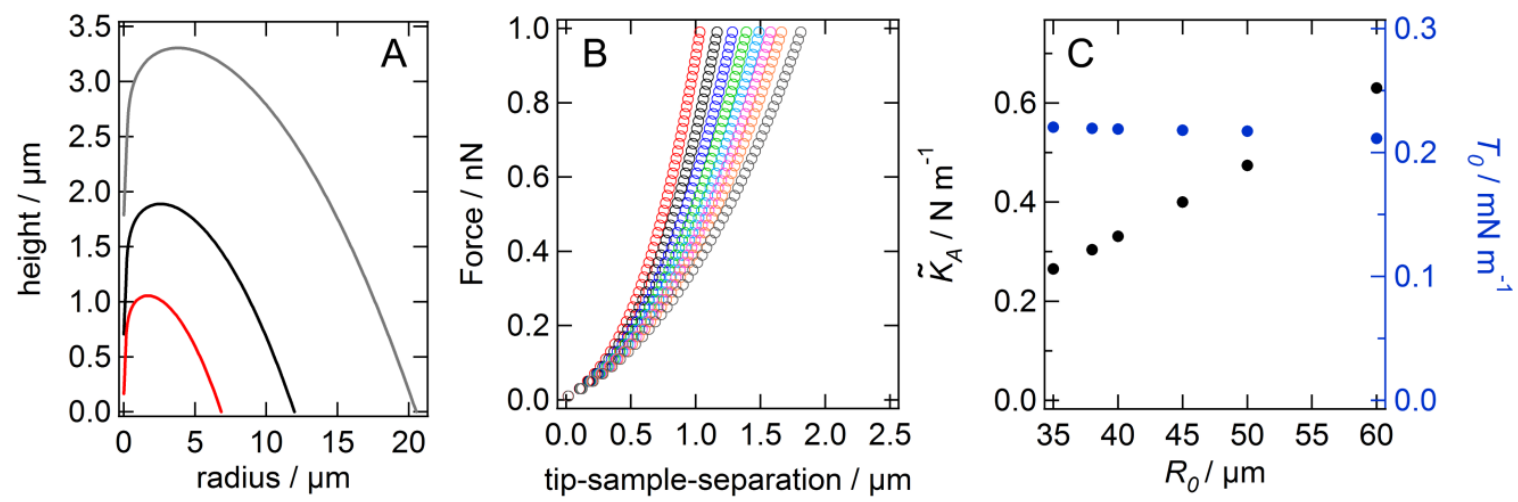

Figure 21: Influence of cellular shape (cell radius) on mechanical parameter. A: Contour of an adherent cell represented by a spherical cap subject to indentation using a conical indenter and a maximal force of $1 \mathrm{nN}$. A wetting angle prior to indentation of $\phi=20^{\circ}$ and an initial cell radius $R_{0}=(-) 20 \mu \mathrm{m}$, (-) $35 \mu \mathrm{m},(-) 60 \mu \mathrm{m}$, (note that $R_{1}=R_{0} \sin (\phi)$ ). A half opening angle of the conical indenter of $\alpha=17.5^{\circ}$ are used for computation. B: Simulated force curves assuming an initial cell radius $R_{0}=(\bullet) 20 \mu \mathrm{m},(\bullet) 25 \mu \mathrm{m},(\bullet) 30 \mu \mathrm{m},(\bullet) 35 \mu \mathrm{m}$, (•) $40 \mu \mathrm{m},(\bullet) 45 \mu \mathrm{m},(\bullet) 50 \mu \mathrm{m},(\bullet) 45 \mu \mathrm{m}$. C: While increasing the wetting angle $\phi$ prior to indentation, the initial cell radius $R_{0}$ also increases. This leads to an increase in $\tilde{K}_{A}$, while $T_{0}$ stays almost constant.

This demonstrates how the apparent area compressibility modulus reflects changes in the geometry of the cell, more precise $A_{0}$. 


\subsection{Mechanical Description of Pulled Nanotubes}

Apart from calculating membrane tension from indentation experiments a second mechanical approach is given by analyzing membrane nanotubes [103] [104] [105] [106]. The first membrane nanoubes or tethers were drawn from red blood cells with attached latex beads with the micropipette aspiration technique [107]. A second method for pulling tether is the magnetic tweezer system, thereby separating a membrane bound bead from the surface [108]. Membrane tubes can also been subtracted from vesicles, showing that tethers can be formed without an underlying cytoskeleton [109].

The formation of membrane tethers can also be achieved with the AFM setup. When an AFM tip is chemically bound to the plasma membrane and afterwards pulled back, a thin bilayer tube or tether is formed (Figure $22 \mathrm{~A}$ ). When the tether ruptures this event is visible in the retrace curve of force-distance cycles by a sharp step, giving the tether force $F$. During this process additional membrane from preexisting reservoirs is flowing into the growing tether. The force on the tether is directly connected to the in-plane tension and bending rigidity of the membrane. In a cell a further contribution arise from the membranecytoskeleton adhesion. The force $F$ (tether force) to pull a tether at constant velocity $v_{t}$ out of a membrane is described by [105] [110]:

$F_{\text {tether }}=2 \pi \sqrt{2 \kappa T_{t}}+\frac{2 \pi \eta v_{t}}{C}$

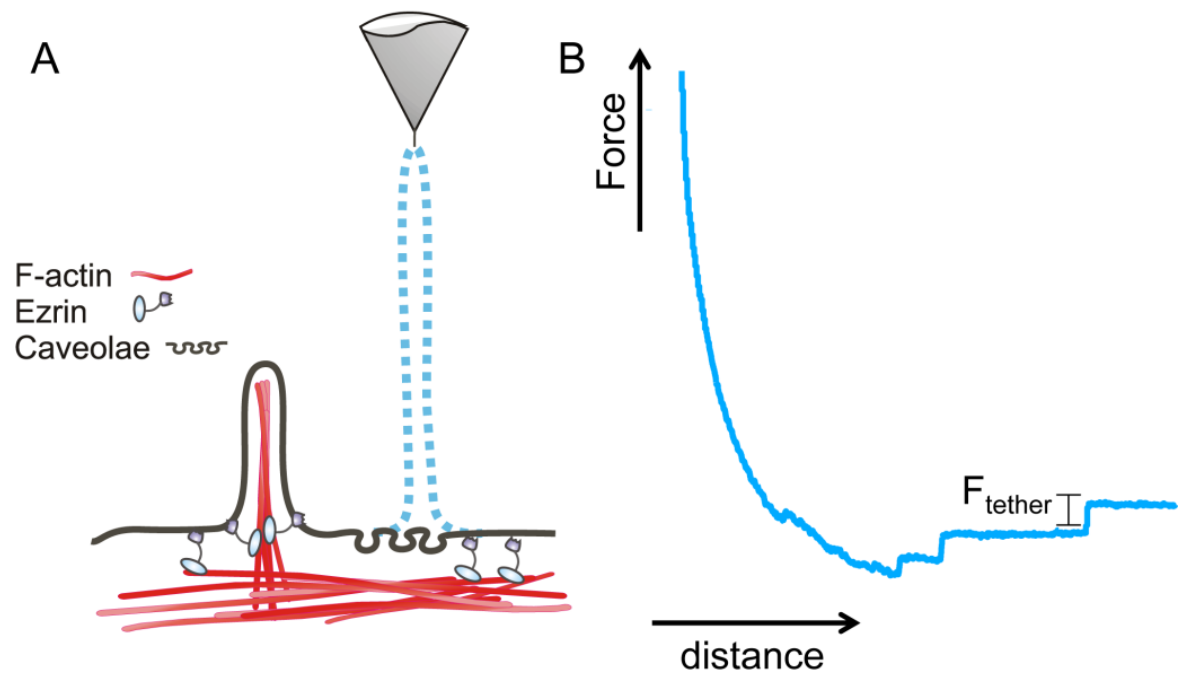

Figure 22: A: Schematic illustration of formation of a membrane tether by pulling a AFM tip from the surface. Membrane shows adhesion to the underlying cortex and membrane reservoirs B: Retrace curve in 
blue displays formation of membrane tethers (force plateau) employed to determine tension $T_{t}$ from Equation 24.

where $\kappa$ is the bending rigidity, $T_{t}$ the membrane in-plane tension and $\eta$ the membrane viscosity, $C$ is a correction factor (1.6) [111]. This Equation can be used to determine the membrane viscosity [112]. For estimating the membrane in-plane tension $T_{t}$, we neglect the viscous contributions as a first approximation. In section 6.1.3 we experimentally determine the viscous part of the tether force.

It is also possible to estimate the bending rigidity of the plasma membrane from membrane tether pulling experiments (section 6.1.4). The experiments from Heinrich and Waugh show nicely that by varying the membrane tension of a vesicle with micropipette aspiration followed by tether pulling with a bead by magnetic tweezers, the tether force increases with the square root of the tension. Additionally the slope of this plot exhibit the bending rigidity [108], as indicated by Equation (32). Bending rigidities of $1.1 \cdot 10^{-19} \mathrm{~J}$ to $2.2 \cdot 10^{-19}$ J, depending on the cholesterol amount, are reported (Waugh et al, 1992).

Knowing the in-plane tension $T_{t}$ and the bending rigidity $\kappa$ of the membrane allows to determine the radius of a tether from the following relationship (section 6.1.4) [105]:

$R_{t}=\sqrt{\frac{\kappa}{2 T_{t}}}$

The radius of a tether varies from small values of $5 \mathrm{~nm}$ found by Hochmuth et al. [113] to larger than $200 \mathrm{~nm}$ reported from Dai and Sheetz [114]. They are observed to decrease when the tension exerted on the membrane increases. 


\section{Experimental}

\subsection{Microinjection - tool of mechanical delivery}

The single-cell microinjection has been successfully used to insert exogenous substances like proteins, cDNA, peptides, drugs or nanoparticles into the cell. It is a common method for cell manipulation in the modern molecular cell biology [115]. The efficiency of the injection system, in other words, the information of the exact concentration inside the cell at a defined time point, is of great importance for the research in biology or the administration and dose of drugs in medicine. In a well defined experiment the only variable is the injected material. Hence, an observed effect can be attributed solely to the injected substance. Injected cells can be recognized in a cell culture when they are coinjected with a membrane impermeable substance (e.g. fluorescence marker like FITCdextran). The microinjection exerts physical stress on the cell, therefore precise and wellconsidered control experiments are required. Single-cell microinjection is time consuming, displaying the disadvantage of a limited number of injected cells in each experiment.

\subsubsection{Setup}

A requirement for the microinjection experiments is the combination of different setups. The microinjection system (eppendorf, Hamburg, Germany) is part of an inverted microscopic devise (Olympus IX81, Tokyo, Japan), which enables fluorescence microscopy in conjunction with phase contrast microscopy. Used objectives and light sources are listed below (Table 3).

Table 3: Objectives and light source used for microinjection experiments

\begin{tabular}{ll}
\hline object & description \\
\hline 10 x objective & UPLFLN 10X2PH, NA = 0.3, Olympus, Tokyo, Japan \\
$\begin{array}{l}40 \text { x objective } \\
\text { phase contrast microscopy } \\
\text { light source }\end{array}$ & 100 W halogen lamp \\
$\begin{array}{l}\text { Fluorescence microscopy } \\
\text { light source }\end{array}$ & X-Cite 120PC Q, Lumen Dynamics Group, Ontario, Canada \\
FITC filter cube & $\lambda$ ex $=490 \mathrm{~nm}$ and $\lambda$ em $=520 \mathrm{~nm}$; Olympus, Tokyo, Japan \\
\hline
\end{tabular}


To observe injected cells under almost culture conditions a petri dish heater (JPK instruments, Berlin, Germany), set to $37^{\circ} \mathrm{C}$, is integrated into the setup. The microinjection system consists of a microinjector (FemtoJet, eppendorf, Hamburg, Germany) and a micromanipulator (InjectMan NI 2, eppendorf, Hamburg, Germany). For the injection commercially available glass capillaries with an inner diameter of $0.5 \mu \mathrm{m}$ are used (Femtotips, eppendorf, Hamburg, Germany).

\subsubsection{How to Inject a Cell}

The correct microinjection of a cell requires several well-considered steps. In this section the observation from our laboratory are summarized to give an overview about this technique.

\section{Before injection}

The right cell line - not every cell line is suitable for injection. When injecting adhering cells, the operator should take care of the cell's height. The microinjection of very flat cells $(<2 \mu \mathrm{m})$ is difficult because of piercing through the cell body. In the case of suspended cells, it is inevitable to use a second manipulator equipped with a capillary to fix the cell.

Choice of substance - the substance, which is injected, should be soluble in a physiological buffer ( $\mathrm{pH} 7$, osmolarity 300 mOsmol). Precipitates have to be prevented in order to avoid clogging of the glass capillary. It is advisable to centrifuge the solution before use. When single cell injection is performed, to provide neighboring cells as direct control, a membrane impermeable substance is recommended.

Medium - Since the microinjection is not performed under culture condition, Hepes buffered medium supplemented with antibiotics and fungicide is necessary.

Petri dish - The microinjection is executed under a certain angle. For microinjecting MDCK II cells the glass capillary is moved $30^{\circ}$ tilted to the substrate. Therefore, petri dishes with low edges are preferable. Besides, a grid on the substrate helps to recognize the injected cells, even after a day. Suitable petri dishes are commercially available ( $\mu$-Dish 35mm Grid-500, ibidi, Martinsried, Germany). 
Device settings - In general, the injection pressure $\left(P_{i}\right)$ and time $\left(t_{i}\right)$ should be adapted to the used cell line, since internal pressure and membrane integrity differs. For MDCK II cells $P_{i}$ and $t_{i}$ was varied until a $90 \%$ survival rate was reached $\left(P_{i}=60 \mathrm{hPa}\right.$ and $\left.t_{i}=0.3 \mathrm{~s}\right)$ (Figure 25). The compensation pressure is regulated until the flow of injection substance out of the capillary is minimal but still visible.

Filling the glass capillary - A filling volume of $5-10 \mu \mathrm{L}$ is sufficient to perform an experiment. It is important to ensure, that no air bubbles appear inside the needle. After filling of the glass capillary it should be immediately mounted into the manipulator and immersed into the petri dish filled with medium. This avoids clogging of the needle.

During injection

Bring the capillary into focus - one advantage for unexperienced users is to find the glass capillary through the microscope and adjusting it in the focal plane of the cells without damaging cells or needle. If a fluorescent substance is co-injected, it is suggested to find the needle in the corresponding fluorescence channel (Figure $24 \mathrm{~B}$ ).

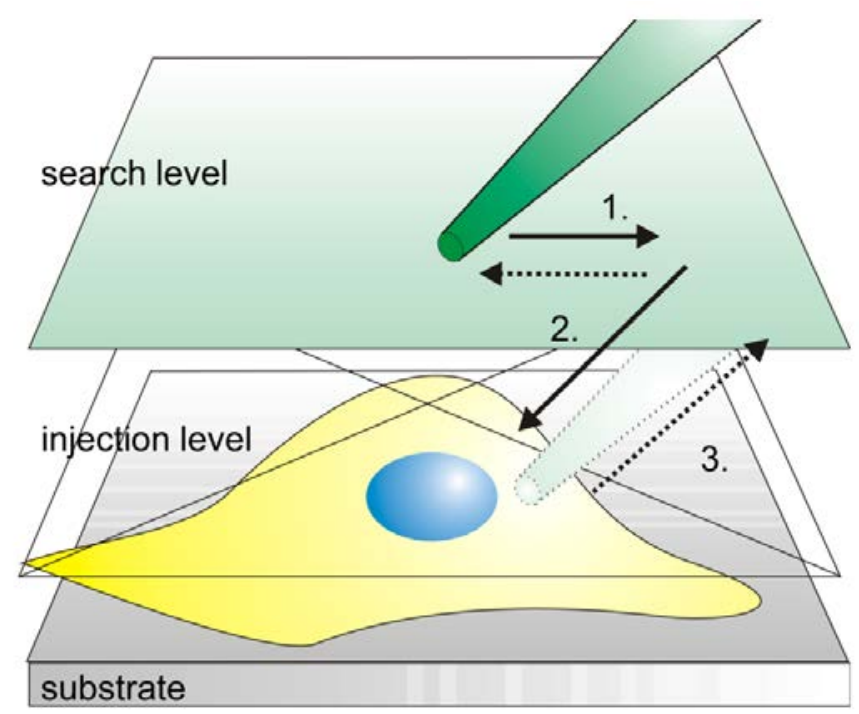

Figure 23: Semi-automatic microinjection. The injection level has to be defined by slight pressing on the cell membrane. After setting the level, the capillary is moved back to the search level. Modified from Brochure InjectMan NI 2 "Injection meets Innovation”.

Inject the cells - the eppendorf set up is also capable for semi-automatic microinjection, which reduces cell damage due to long and harsh injection. In particular, the movement of the capillary illustrated in Figure 23 (step 1-3) is performed automatically. Before starting the semi-automatic injection the injection level has to be defined. The capillary is therefore brought into contact with the cellular membrane until a white hallow is visible around the 
needle. Then the limit is set and the capillary is moved back above the cells to the search level. When the needle penetrates the membrane a gentle wave should pass through the cell from the site of microinjection. If this wave is to hard the cell will blow up and will not survive (Figure $24 \mathrm{~A}$ ). If this is the case, the injecting pressure or injection level should be changed. For cells growing in a confluent layer and displaying a similar height, the injection level can be kept constant.
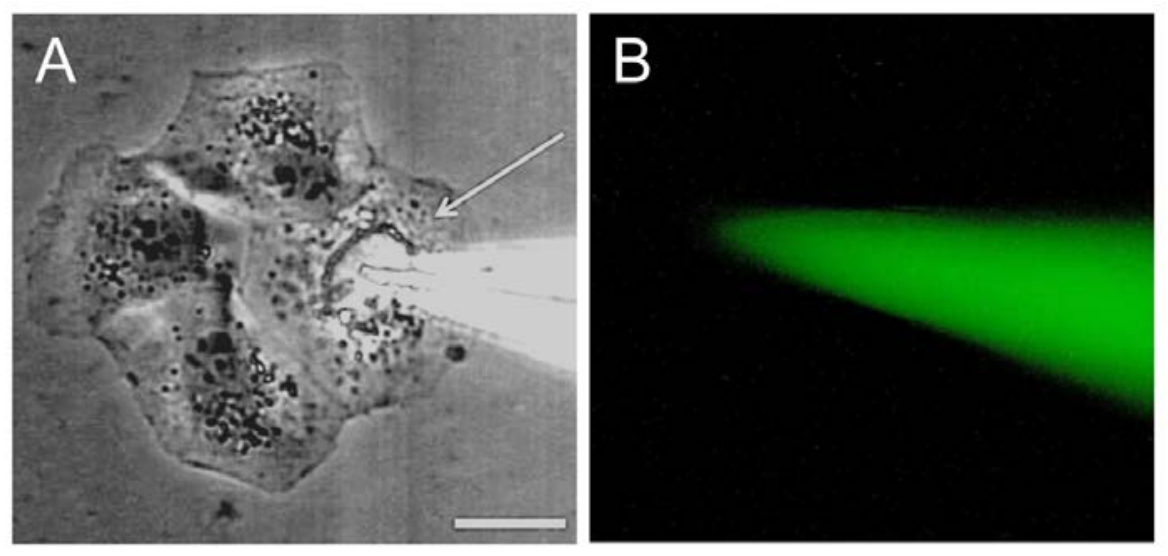

Figure 24: (A) Phase contrast micrograph of MDCK II cell during microinjection. Injected cell blows up and loses contrast. Scale bar is $10 \mu \mathrm{m}$. (B) Fluorescence micrograph of FITC-dextran filled glass capillary.

Check injection - as the capillary clogs very fast, the accuracy of the microinjection should be controlled. Therefore, the co-injected fluorescent marker can be monitored in the fluorescence channel. When the cells are not fluorescently marked, it can be assumed that the injection failed and should be repeated, after the compensatory pressure has been increased.

\section{After injection}

Since the microinjection exerts physical stress on the cells, surveillance of viability of injected cells is mandatory. A propidium-iodide staining can be performed, which visualizes loss of membrane integrity by staining the nucleus red (Figure 25).

When not suitably injected, cells display huge blebs. This is a distinctive feature for a membrane-cortex detachment. Cells showing these membrane outgrowths are not considered for experiments (Figure 26). 

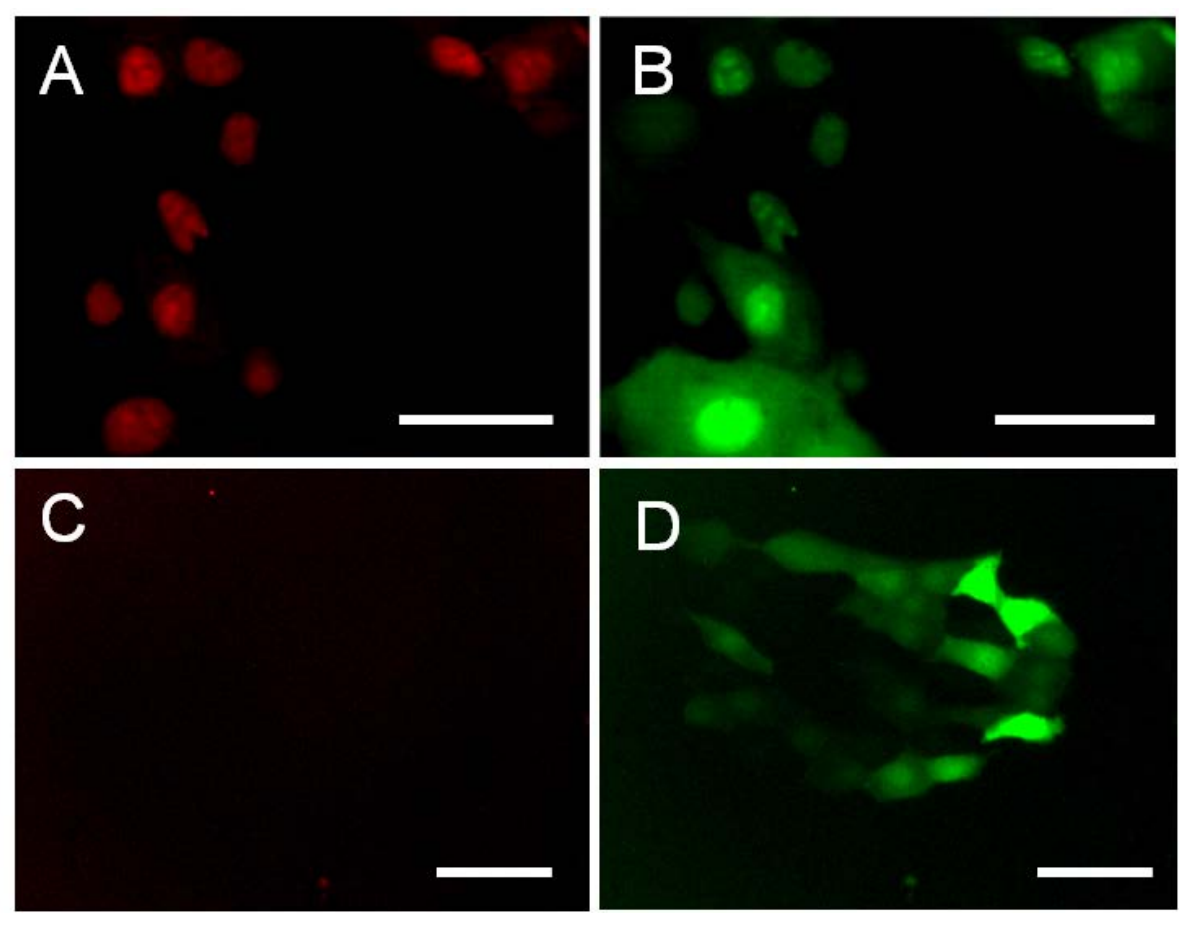

Figure 25: Evaluation of the quality of the performed microinjection. A: Fluorescence micrograph of a propidium-iodide-staining of FITC-dextran injected cells (B) high injection parameter $P_{i}=150 \mathrm{hPa}$ and $t_{i}=$ $0.5 \mathrm{~s}$. Scale bar is $20 \mu \mathrm{m}$. Red nuclei display loss of membrane integrity. C: Red staining of the nuclei is missing for injection parameter of $P_{i}=60 \mathrm{hPa}$ and $t_{i}=0.3 \mathrm{~s}(\mathrm{D})$. Scale bar is $20 \mu \mathrm{m}$.
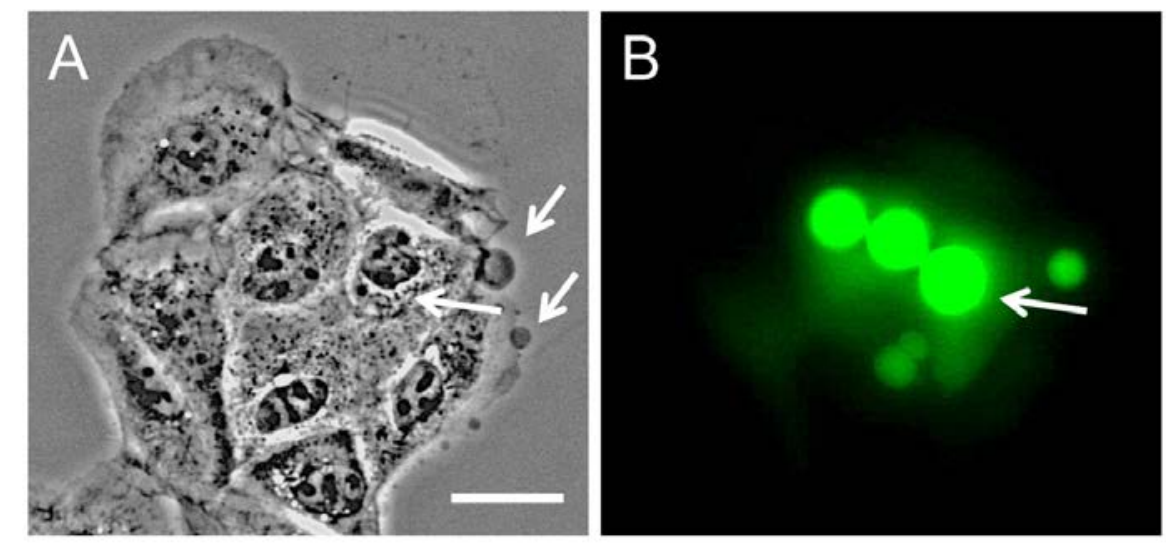

Figure 26: MDCK II cells display blebs after microinjection. A: Phase contrast micrograph. Arrow display blebs. Scale bar $10 \mu \mathrm{m}$. B: Fluorescence micrograph of FITC-dextran injected cells. Huge blebs are only visible in the fluorescence image.

Cells are successfully injected when they display no change in contrast and morphology (Figure 27). To ensure whether the cells are damaged due to injection or not, the cells are observed for $1 \mathrm{~h}$. Thereafter, the cells are considered to be suitable further experiments. 


\subsubsection{Injected Substances}

\subsubsection{Neomycin}

For the mechanical investigation of membrane-cytoskeleton adhesion neomycin, a cationic antibiotic known for specifically forming complexes with $\mathrm{PIP}_{2}$ [116], is microinjected into confluent MDCK II cells (25 mg/mL in PBS, Sigma Aldrich, Germany). FITC-dextran (5 $\mathrm{mg} / \mathrm{mL}$ in PBS, Sigma Aldrich, Germany, MW = 70,000) served as co-injection substance, to recognize the injected cell.

\subsubsection{2 $\mathrm{PIP}_{2}{ }^{1}$}

For the microinjection of $\mathrm{PIP}_{2}$ vesicles, $\mathrm{PIP}_{2}$ from the pork brain (Avanti Polar lipids, Alabaster, USA) is used. Because of possible hydrolysis by phospholipase C (PLC) in the cytoplasm after microinjection, a metabolically stabilized analogue of $\mathrm{PIP}_{2}$ is added to the injection solution ( $\alpha$-Fluorophosphonat-PIP2, $\alpha$-F-PIP2, Echelon Bioscience, Salt Lake City, USA) [117]. FITC-dextran serves as fluorescent co-injection substance in a final concentration of $5 \mathrm{mg} \mathrm{mL}^{-1}$ (MW: 70,000 $\mathrm{g} \mathrm{mol}^{-1}$, Sigma-Aldrich, Steinheim, Germany). All three substances are mixed together (total amount of lipid is $25 \mu \mathrm{M}$ ) and for homogenization mixture is put into a ultrasonic bath (50 W, $0.4 \mathrm{~s}, 30 \mathrm{~min})$ [118]. This method yields $\mathrm{PIP}_{2}$-micelles with a hydrodynamic radius of $13 \mathrm{~nm}$, characterized by dynamic light scattering (ALV/CGS-3 Goniometer System, ALV, Langen, Germany; with a $22 \mathrm{~mW}$ He-Ne-Laser ( $\lambda=632,8 \mathrm{~nm}$, JDS Uniphase, Eningen, Germany)).

\subsubsection{FITC-dextran}

Control experiments for proving the influence of the microinjection on the mechanical properties of the cell are performed by injecting FITC-dextran (MW: $70000 \mathrm{~g} \mathrm{~mol}^{-1}$, Sigma-Aldrich, Steinheim, Germany) at a concentration of $5 \mathrm{mg} / \mathrm{mL}$ dissolved in PBS ${ }^{--}$ into the cytoplasma of the cells (Biochrom AG, Berlin, Germany).

\footnotetext{
${ }^{1}$ Experiments and data processing was carried out by Bastian R. Brückner during his Master Thesis.
} 


\subsection{Atomic Force Microscopy}

In 1986 Binnig et al. introduce a new setup for the investigation of insulating surfaces, the atomic force microscope (AFM) [119]. It was an advancement of the scanning tunneling microscope (STM) and enables with its different modi beside topological examination of a surface the analysis of the mechanical properties of a sample. The advantage of this technique lies in the mechanical principle, which detects forces without being too invasive.

The heart of the AFM is a sharp tip attached to a flexible beam in short distance to the sample. The tip-sample separation is, as well as the lateral position set by a piezoelectric element, which allows the precise lateral scanning of the surface. The deflection of the beam is thereby monitored with an optical lever method [120]. For further details please refer to [121].

\subsubsection{AFM for Imaging}

All images shown in this work are recorded in contact mode. Further imaging modes are the non-contact and intermittent contact mode [121]. In the contact mode the tip is in permanent contact with the sample. Repulsive forces between the tip and the sample cause a deflection of the beam. This deflection is compensated by the movement of the piezoelectric element and the acting force between tip and sample is kept constant according to Hooke's law.

$F=-k d$

The cantilever deflection $d$ is converted into a force $F$ by multiplying it with the spring constant of the cantilever $k$. The photodiode signal gives the deflection image (Figure 7 B). The z-piezo-control yield height information (Figure 27 A) and by scanning in xy-direction a three-dimensional topological image of the surface is generated.

The advantage of the contact mode is the control of the force acting on the sample. Using "soft" cantilever (beam) with a spring constant smaller than $50 \mathrm{mN} \mathrm{m}^{-1}$ reduces damages to the samples. This is a requirement when imaging soft living cells.

Here, the technique is used to image the surface of MDCK II cells. Additionally, the topological information, especially height data are used to modeling the spherical cap for the mechanical approach (section 3.2.3). 


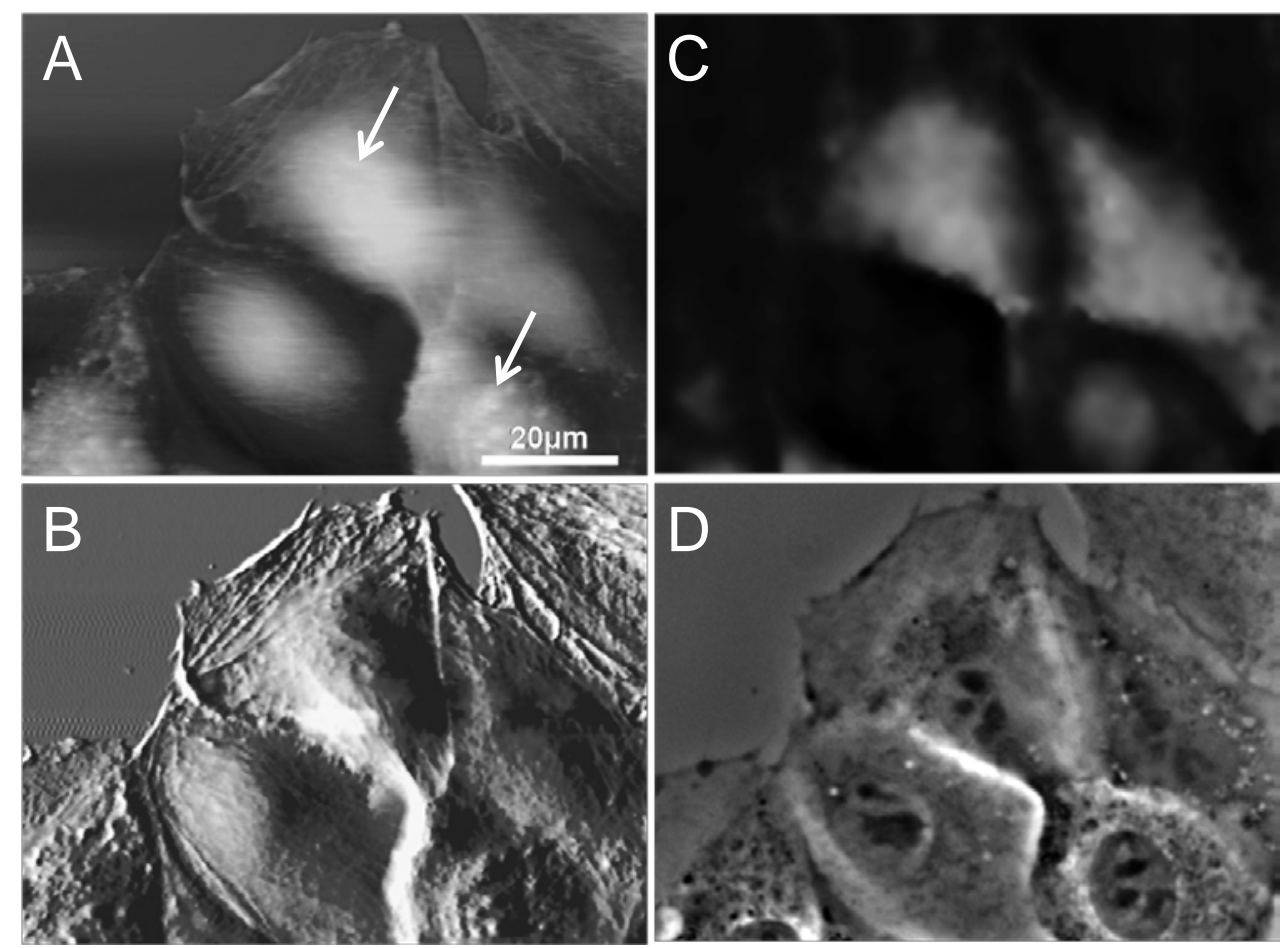

Figure 27: Subconfluent MDCK II cells are imaged with the AFM after microinjection. A: AFM height image. Arrows indicate structural changes of injected cell (upper arrow) and unsuccessfully microinjected cell (lower arrow). B: AFM deflection image showing details of the surface, like stress fibers and plasma membrane protrusions. C: Fluorescence micrograph of FITC-dextran injected cells. D: Phase contrast micrograph of the same position as in $\mathrm{A}, \mathrm{B}, \mathrm{C}$.

During the manipulation of the cellular mechanics it is expected that density and structure of tension buffering membrane reservoirs like microvilli are altered. This is visualized with high resolution AFM images. As evident in Figure 27, the difference in injection quality is only observable in the AFM images. Fluorescence as well as phase contrast micrographs alone do not display the structural differences as observed in the AFM images. An overall picture of the microinjection quality can only be drawn by considering all imaging techniques.

AFM imaging is performed with a Nanowizard ${ }^{\circledR}$ II and III AFM (JPK Instruments AG, Berlin) mounted on an Olympus IX 81 inverted light microscope. Although AFM delivers images of unprecedented resolution under physiological conditions, fixation of the plasma membrane for high resolution imaging of confluent cells is inevitable due to membrane flexibility and lateral mobility. Therefore, cells are generally fixated using $2.5 \%$ glutardialdehyde in PBS added to cells for $20 \mathrm{~min}$. Silicon nitride cantilevers (MLCT, Bruker AFM Probes, Camarillo, USA) with a nominal force constant of $0.01 \mathrm{~N} \mathrm{~m}^{-1}$ are employed. The exact spring constant is determined with the thermal noise method 
integrated into the software of the instrument [121]. Cells were imaged in PBS at room temperature with a scan rate of $0.2 \mathrm{~Hz}$ and processed with JPK Image Processing® software.

For imaging living cells, HEPES buffered culture medium supplemented with antibiotics and fungicides, is used (penicillin $\left(0,2 \mathrm{mg} \mathrm{mL}^{-1}\right.$, PAA, Pasching, Austria), streptomycin (0,2 mg mL ${ }^{-1}$, PAA, Pasching, Austria), und amphotericin B (5 $\mu \mathrm{g} \mathrm{mL}^{-1}$, PAA, Pasching, Austria)). HEPES is supplemented in a concentration of $15 \mu \mathrm{M}$ (PAA, Pasching, Austria). The sample is placed in a petri dish heater (JPK instruments, Berlin, Germany) set to $37^{\circ} \mathrm{C}$.

Methyl- $\beta$-cyclodextrin treatment (MBCD)

Cells were grown in Ibidi $^{\mathrm{TM}}$ petri dishes (Ibidi, Martinsried, Germany) to confluency and treated with $10 \mathrm{mM}$ methyl- $\beta$-cyclodextrin (Sigma Aldrich, Germany) for 1-3 hours. For imaging cells were fixed with 2.5\% glutaraldehyd in PBS for 20 min.

Neomycin and $\mathrm{PIP}_{2}$ Injection

Cells are imaged after neomycin and $\mathrm{PIP}_{2}$ injection to investigate the alteration in the density of membrane protrusions. Prior to fixation with 2.5\% glutaraldehyd in PBS for 20 min, cells are mechanically examined.

\section{Osmotic Stress}

To visualize the structural changes upon osmotic stress of the plasma membrane of MDCK II cells, cells are grown to confluency on Ibidi $^{\mathrm{TM}}$ petri dishes (Ibidi, Martinsried, Germany) and subjected to different osmotic solutions. After 30 min cells are fixed for 30 min with 2.5\% glutaraldehyd in PBS.

Table 4: Osmotic solutions:

\begin{tabular}{ccccc}
\hline & control & mild hypotonic & strong hypotonic & hypertonic \\
\hline osmolality [mOsm] & 308 & 200 & 80 & 600 \\
\hline
\end{tabular}

Solutions are prepared by diluting the medium with appropriate amount of distilled water. For the hypertonic solution glucose is added to the medium in a final concentration of 400 $\mathrm{mM}$. The osmolarity of the used solutions is measured with cryoscopy osmometer (OSMOMAT 030, Gonotec). 


\subsubsection{AFM for Elasticity Measurements}

Despite the capability of high-resolution imaging, the AFM is also suitable for sensitive force measurements. Force resolution is ultimately limited by thermal motion of the cantilever beam. In force spectroscopy measurements the cantilever is vertically approached to the sample surface, rather than laterally as in the scanning mode, and after contact with the surface (contact force and time can be adjusted) retracted back by applying voltage to a piezoelectric-element. During this movement the cantilevers deflection is recorded, relative to its displacement (Figure 28). The deflection of the cantilever is thereby measured by an optical method (in Volts, signal from the diode detector.

A

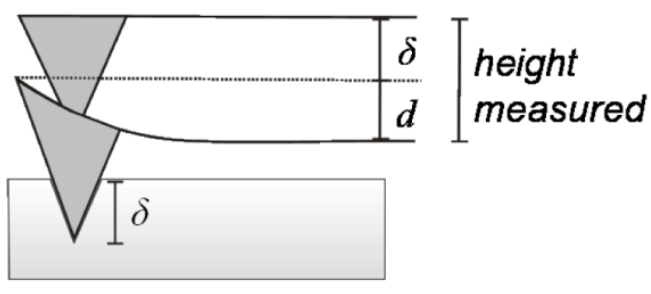

B

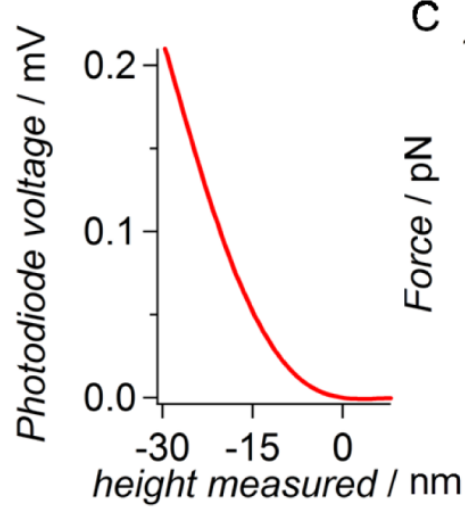

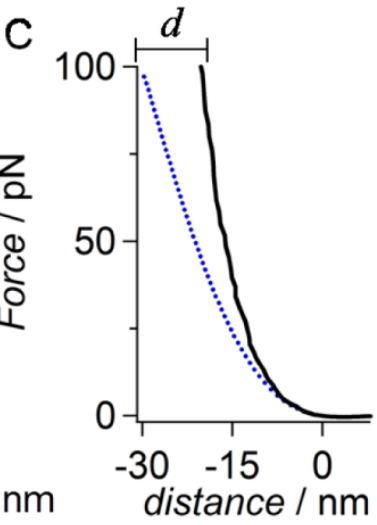

Figure 28: Illustration of an indentation experiment on a hard surface required for data processing. A: Cantilever is moved in the direction of the sample by a certain distance (height measured) and is bend in the opposite direction $(d)$ while the sample is indented by $\delta$. B: Force curve in its raw form: detector signal $(\mathrm{mV})$ versus piezo displacement (nm). C: After determine the sensitivity of the system and calculating the spring constant $k$, the force distance curve (black) is obtained. Dashed blue curve represents force versus height measured.

This signal is converted into a force by measuring the sensitivity of the system (translating “in Volts" into a distance) and the spring constant of the cantilever by the thermal noise method, according to Hooke's Law (Equation 34).

For quantitative analysis of the indentation experiments the displacement of the piezo has to be converted into a true tip sample separation. This is done by subtracting the deflection due to bending of the cantilever from its equilibrium position (Figure $28 \mathrm{C}$, blue curve is converted into black curve). This yields force distance curves. Force Mapping is an extension of the force spectroscopy mode. In this mode a defined number of single force spectroscopy experiments in a distinct area are performed. Usually, an image of the sample 
surface is recorded and than overlayed with a grid of spectroscopic points (Figure 29). Force distance curves are taken continuously at each point in a scan area consisting of $32 \mathrm{x}$ 32 pixels. This data recording results in a map displaying local mechanical properties of the sample (Figure 29).

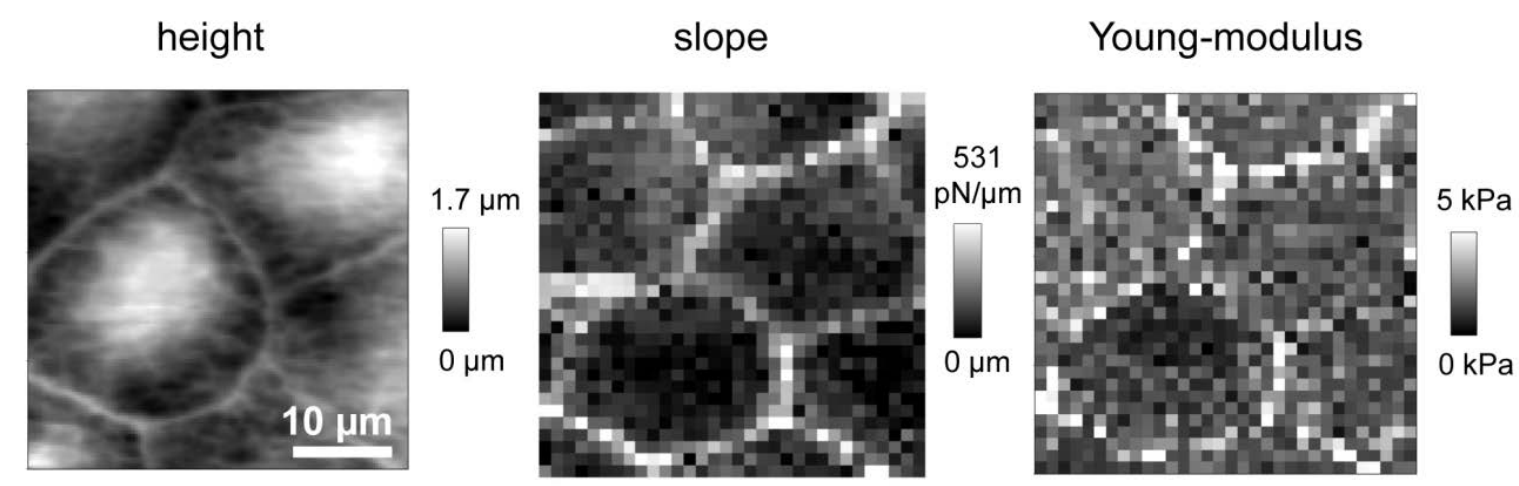

Figure 29: Force mapping experiment of living MDCK II cells. (Left) AFM height image. First an image is scanned to define the area for spectroscopy experiment. Force distance curves are taken continuously at each pixel. (Center) Slope of the force distance curves. (Left) Hertzian mechanical model is fitted to force curves yielding a Young's modulus map.

The advantage of this mode arises from the possibility to record local site-specific elastic properties creating a mechanical image of the sample (Figure 29). When a cell is microinjected, this spectroscopic mode enables to detect mechanical changes of the cells in coincidence with neighboring untreated cells. After collecting the data mechanical models can be fitted to the force curves (section 3), which reveals mechanical parameter as the Young's modulus $E$, the membrane tension $T_{0}$ and the apparent area compressibility modulus $\tilde{K}_{A}$.

Force curves are taken continuously using a Nanowizard ${ }^{\mathrm{TM}}$ II and III AFM (JPK instruments AG, Berlin, Germany) while scanning laterally across the sample referred to as force mapping [122, 123]. Cantilevers with a nominal spring constant of $0.01 \mathrm{~N} \mathrm{~m}^{-1}$ (MLCT, Bruker AFM, Proes, Camerillo, USA) are used for all AFM spectroscopy experiments. The opening angle $\alpha$ of the tip indenter is $17.5^{\circ}$. Measurements are performed with an approach/retraction velocity of $2 \mu \mathrm{m} \mathrm{s}^{-1}$, and a set point of $1 \mathrm{nN}$. MDCK II cells are seeded on Ibidi ${ }^{\mathrm{TM}}$ petri dishes (ibidi, Martinsried, Germany) grown to confluency and mounted in a PetriDishHeater ${ }^{\circledR}$ (JPK instruments AG, Berlin, Germany) set to $37{ }^{\circ} \mathrm{C}$ and filled with HEPES buffered culture medium. The medium is supplemented with antibiotics and fungicides, is used (penicillin $\left(0.2 \mathrm{mg} \mathrm{mL}^{-1}\right.$, PAA, Pasching, Austria), streptomycin 
(0.2 mg mL ${ }^{-1}$, PAA, Pasching, Austria), und amphotericin B (5 $\mu \mathrm{g} \mathrm{mL}^{-1}$, PAA, Pasching, Austria)). HEPES is supplemented in a concentration of $15 \mu \mathrm{M}$ (PAA, Pasching, Austria). For mechanical investigation of adhering and spreading cell single force curves are taken in the middle of a single cell.

\subsubsection{AFM for Probing Tether Forces}

As a second mechanical approach membrane nanotubes are pulled from the cellular surface. Tether forces are obtained from retrace curves of force cycles as described in section 3 (Figure 22). From the force plateau $\left(F_{\text {tether }}\right)$ of a fully established membrane nanotube, we infer the membrane tension $T_{t}$ according to [106, 124]:

$F_{\text {tether }}=2 \pi \sqrt{2 \kappa T_{t}}$

For computing $T_{t}$ we chose a bending module $\kappa$ of $10^{-19} \mathrm{~J}$ representing a fluid lipid bilayer. We found that viscous contributions to the tether force could be neglected as a first approximation for reasons described in section 5.1.3.

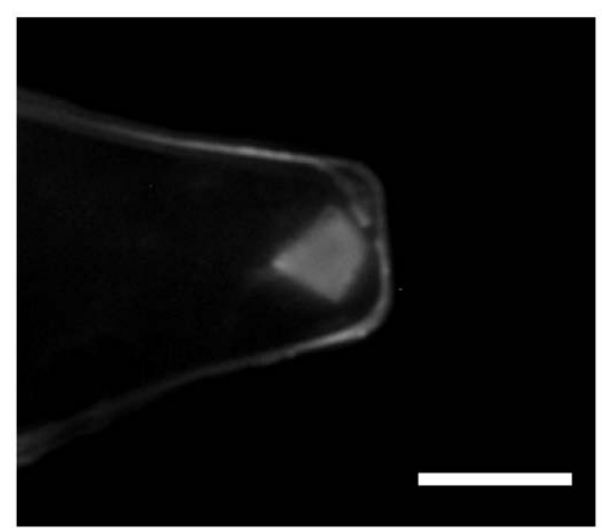

Figure 30: Fluorescence micrograph of a FITC-concanavalin A coated MLCT-cantilever to visualize coating efficiency. Scale bar $10 \mu \mathrm{m}$.

Prior to tether pulling, cantilevers (MLCT, Bruker AFM, Proes, Camerillo, USA) are plasma cleaned for $30 \mathrm{sec}$ (Argon) and incubated with $2.5 \mathrm{mg} / \mathrm{mL}$ concanavalin A (Sigma Aldrich, Germany) in PBS for $1.5 \mathrm{~h}$, as lectins are known for a strong binding to carbohydrates on the cellular surface to provide strong adhesion to the membrane and thereby extracting membrane nanotubes upon retraction of the tip. Both approach and retrace velocities were set to $2 \mu \mathrm{m} / \mathrm{s}$. Tether pulling experiments are simultaneously performed with indentation experiments. Special sample preparation is not necessary. 


\subsection{Microscopy}

To ensure the viability of the cells, all mechanical measurements are accompanied by microscopic device. Therefore the AFM set up is mounted on an inverted microscope (Olympus IX81, Tokyo, Japan), which enables fluorescence microscopy in conjunction with phase contrast microscopy.

Besides, fluorescence microscopy is performed to visualize intracellular structures like the cytoskeleton, membrane-cytoskeleton linker protein, cell-cell contact and others (section 4.3.3) whose alteration would lead to a change in mechanical properties.

\subsubsection{Phase Contrast Microscopy}

Cells display low contrast in bright field microscopy and appear almost transparent. For methods relying on the visibility of the cellular structure like microinjection, contrast enhancing techniques are required.

The most common one is the phase contrast microscopy. The human eye only detects differences in intensity and color, phase changes due to objects must therefore be converted into intensity differences. The phase contrast technique translates variations in phase of transmitted light into corresponding changes in amplitude. This leads to differences in the image contrast. Requirements for this technique are the microscope alignment for Köhler Illumination and the correct alignment of the phase plate at the condensor and the phase ring inside the objective [125].

Used objective lenses are listed in Table 3.

\subsubsection{Fluorescence Microscopy}

Changes in the cellular structure are visualized by immunostaining and fluorescence microscopy. For this purpose, a fluorescence microscope (BX 51, Olympus, Tokyo, Japan) with water immersion objectives $40 \mathrm{x}$ (LUMPLFLN 40XW, NA = 0.8, Olympus, Tokyo, Japan) and $100 \mathrm{x}$ (LUMPLFL 100XW, NA = 1.0, Olympus, Tokyo, Japan) is used. The light source is a mercury vapor lamp (X-Cite 120Q, Lumen Dynamics Group, Ontario, Canada). 


\subsubsection{Cell Labeling}

For all application except experiments on adhering and spreading cells, cells are grown onto Ibidi $^{\mathrm{TM}}$ petri dishes (Ibidi, Martinsried, Germany) to confluency and after required treatment fixed with $4 \%$ paraformadehyde PFA for $20 \mathrm{~min}$. Cells in suspension were seeded in Ibidi ${ }^{\mathrm{TM}}$ petri dishes (Ibidi, Martinsried, Germany) and incubated for different time intervals (5, 15, 30 min). Cells were fixed with 4\% paraformadehyde PFA for 30 min. For the staining procedure, detailed substance description is listed in Table 5.

For nucleus staining DAPI (4',6-Diamidino-2-phenylindol) is diluted as listed in Table 5 in dilution buffer added to the sample and incubated for $10 \mathrm{~min}$ at room temperature. After treatment cells are washed three times with $\mathrm{PBS}^{--}$.

F-actin labeling is carried out with Alexa 546- or 488-phalloidin diluted as recommended by the manufacturer in dilution buffer. After 45 min samples are washed three times with PBS.

Secondary antibody staining procedure (Ezrin, $\mathrm{PIP}_{2}$, Integrins and ZO-1)

After fixation with 4\% paraformadehyde (PFA) for $20 \mathrm{~min}$ at room temperature, cells are washed three times with PBS $^{--}$. For blocking unspecific binding sites and permeabilization of the membrane, cells are treated with blocking buffer for 30 min (composition listed in Table 5) and rinsed three times with PBS $^{--}$.

Primary antibody is diluted as described in Table 5 in dilution buffer and incubated with the sample for $1 \mathrm{~h}$ at room temperature. Thereafter the sample is rinsed three times for 5 min with $\mathrm{PBS}^{--}$on a vibratory plate (80 rpm, GFL, Burgwedel, Germany). The secondary antibody is diluted as recommended in Table 5 in dilution buffer and added to the cells for $45 \mathrm{~min}$, followed by rinsing with PBS (three times) for 5 min on a vibratory plate.

Membrane labeling of living cells was carried out by incubating cells for 1 min with PKH2-linker dye (5 $\mu \mathrm{M}$ in PBS) (Sigma Aldrich, Germany). After incubation, cells are rinsed with warm HEPES buffered MEM-medium and mounted under the microscope with temperature control $\left(37^{\circ} \mathrm{C}\right)$.

Spreading cells are fixed prior to membrane labeling to avoid tearing off the weak adhered cells by subsequent rinsing steps. 
Table 5: Antibodies and reagents for immunostaining.

\begin{tabular}{|c|c|c|c|}
\hline structure & antibody & manufacture & dilution \\
\hline Nucleus & DAPI & $\begin{array}{l}\text { Invitrogen, Life Technologies, } \\
\text { Carlsbad, USA }\end{array}$ & $50 \mathrm{ng} \mathrm{mL}^{-1}$ \\
\hline F-actin & $\begin{array}{l}\text { Alexa Fluor 488-Phalloidin } \\
\text { Alexa Fluor 546-Phalloidin }\end{array}$ & $\begin{array}{l}\text { Invitrogen, Life Technologies, } \\
\text { Carlsbad, USA }\end{array}$ & $165 \mathrm{nM}$ \\
\hline Ezrin & $\begin{array}{l}\text { 1. IgG anti-mouse } \\
\text { 2. Alexa Fluor488- } \\
\text { conjugated goat-anti- } \\
\text { mouse IgG1 }\end{array}$ & $\begin{array}{l}\text { 1. BD Biosciences, Heidelberg, } \\
\text { Germany } \\
\text { 2. Invitrogen, Life Technologies, } \\
\text { Carlsbad, USA }\end{array}$ & $\begin{array}{l}4 \mu \mathrm{g} \mathrm{mL}^{-1} \\
4 \mu \mathrm{gL}^{-1}\end{array}$ \\
\hline $\mathrm{PIP}_{2}$ & $\begin{array}{l}\text { 1. Typ Mouse IgG2b } \\
\text { 2. Alexa Fluor546- } \\
\text { conjugated goat-anti- } \\
\text { mouse IgG2 }\end{array}$ & $\begin{array}{l}\text { 1. Enzo Life Sciences, Germany) } \\
\text { 2. Invitrogen, Life Technologies, } \\
\text { Carlsbad, USA }\end{array}$ & $25 \mu g \mathrm{~mL}^{-1}$ \\
\hline ZO-1 & $\begin{array}{l}\text { 1. Typ IgG1 mouse } \\
\text { 2. Alexa Fluor488- } \\
\text { conjugated goat-anti- } \\
\text { mouse IgG1 }\end{array}$ & $\begin{array}{l}\text { 1.Zymed GmbH, Munich, } \\
\text { Germany } \\
\text { 2. Invitrogen, Life Technologies, } \\
\text { Carlsbad, USA }\end{array}$ & $\begin{array}{l}4 \mu \mathrm{gL}^{-1} \\
4 \mu \mathrm{gL}^{-1}\end{array}$ \\
\hline Integrin $\alpha \mathrm{V}$ & $\begin{array}{l}\text { 1. Typ IgG1 mouse } \\
\text { 2. Alexa Fluor488- } \\
\text { conjugated goat-anti- } \\
\text { mouse IgG1 }\end{array}$ & $\begin{array}{l}\text { 1.BD Biosciences, Heidelberg, } \\
\text { Germany } \\
\text { 2. Invitrogen, Life Technologies, } \\
\text { Carlsbad, USA }\end{array}$ & $\begin{array}{l}4 \mu \mathrm{gL}^{-1} \\
4 \mu \mathrm{g} \mathrm{mL}\end{array}$ \\
\hline Membrane & PKH2-linker dye & Sigma Aldrich, Germany & $5 \mu \mathrm{M}$ in PBS $^{-}$ \\
\hline Blocking buffer & $\begin{array}{l}5 \%(\mathrm{w} / \mathrm{v}) \mathrm{BSA}, 0.3 \%(\mathrm{v} / \mathrm{v}) \\
\text { TriX in } \mathrm{PBS}^{-}\end{array}$ & Sigma Aldrich, Germany & \\
\hline Dilution buffer & $\begin{array}{l}1 \%(\mathrm{w} / \mathrm{v}) \mathrm{BSA}, 0.3 \%(\mathrm{v} / \mathrm{v}) \\
\text { TriX in } \mathrm{PBS}^{--}\end{array}$ & Sigma Aldrich, Germany & \\
\hline
\end{tabular}




\subsubsection{Scanning Electron Microscopy}

Scanning electron microscopy (SEM) is used to visualize the surface structure of the plasma membrane of MDCK II cells in a non invasive manner. Therefore cells are grown to confluency on $12 \mathrm{~mm}$ round coverslips and fixed for $1 \mathrm{~h}$ with $2.5 \%$ glutaraldehyd. The fixative is removed and rinsed three times with PBS. Afterwards the sample is immersed in 10\%, 25\%, 50\%, 75\%, 95\% ethanol for each $30 \mathrm{~min}$. Finally the sample is covered with $100 \%$ ethanol over night. After this dehydration steps the sample are dried in a $\mathrm{N}_{2}$-flow and coated with a $15 \mathrm{~nm}$ thick gold layer. Cells are examined with a Leo Supra 55VP SEM (Zeiss, Oberkochen, Germany) at a voltage of $200 \mathrm{kV}$.

For visualizing surface structure after cholesterol depletion cells are prior to fixation treated for 3h with MBCD (10 mM, Sigma Aldrich, Germany).

The cellular cortex is prepared by incubating the cells with $4 \%$ TriX-solution for 30 min, to remove the plasma membrane. Afterwards samples are treated as mentioned above.

\subsection{Electric Cell-Substrate Impedance Sensing}

Electric cell-substrate impedance sensing (ECIS) measures the complex impedance of cells cultured on ultra small gold electrodes, which allows monitoring morphological changes of cells induced by biological, chemical or physical stimuli in real time [126, 127].

The impedance originating from adherent cells as a function of frequency can be roughly split in three regimes describing different cellular properties. The major component of the current at low frequencies $(<10 \mathrm{kHz})$ is flowing paracellular through the cell-substrate adhesion zone characterized by the parameter $\alpha$ and between the cells through intercellular gaps. This barrier resistance $R_{b}$ is mainly determined by the cell-cell contacts or tight junctions of the polarized epithelial cells. Accordingly, the readout of the impedance at these low frequencies is sensitive to changes in cell-substrate contacts and in the density of cell-cell contacts yielding important information about the electrical and mechanical integrity of the cell layer. At higher frequencies $(>10 \mathrm{kHz})$ the transcellular impedance is dominated by the capacitance $C_{m}$, which represents the specific capacity of the plasma membrane [128]. $C_{m}$ provides direct information about the area of the cell surface including membrane protrusions and invaginations. A reduction in $C_{m}$ is indicative of a 
decrease in membrane area given that the electrode coverage remains constant. Generally, a larger coverage corresponds to reduction in $C_{m}$. Analyzing frequency scans over time provides the electrical parameters $R_{b}, \alpha$, and $C_{m}$ in a time resolved manner corresponding to the dynamics of cell morphology and cell layer integrity.

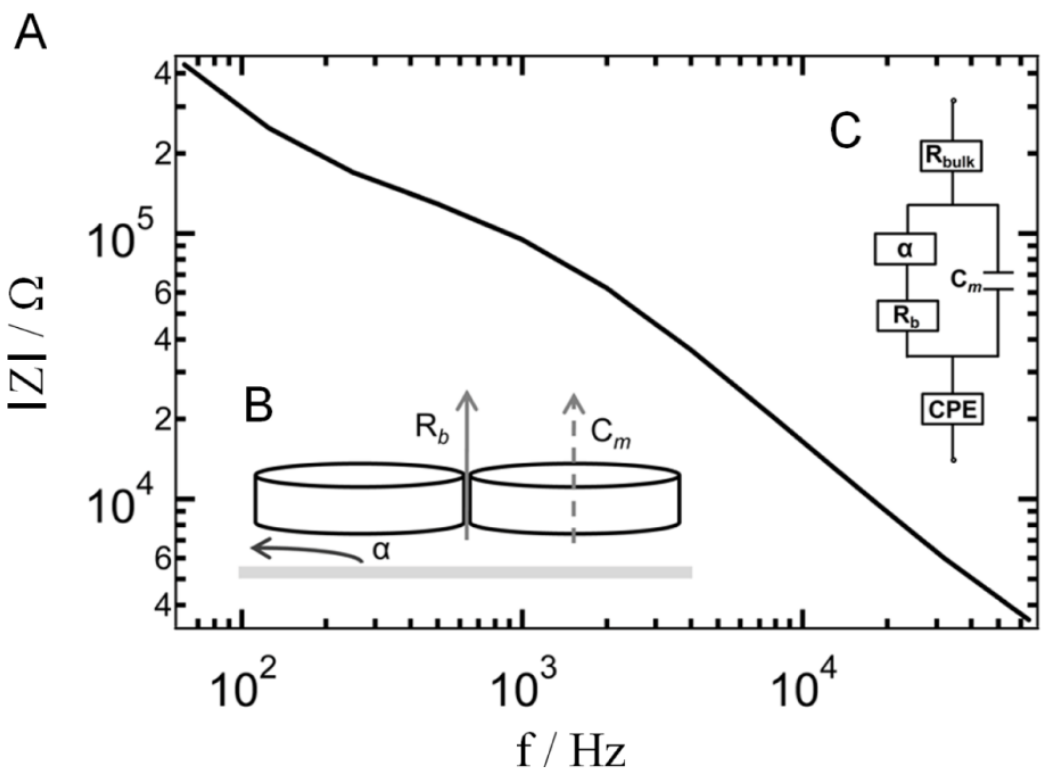

Figure 31: A: Impedance as a function of frequency obtained from a frequency scan measurement for an electrode covered with confluent MDCK II cells. B: Schematic illustration of the ECIS contact area model introduced by Giaever and Keese. Cells are represented by insulating particles with a certain distance to the gold electrode. The solid arrow indicates the current passing the intercellular gap $\left(R_{b}\right)$, while the curved arrow marks the current flowing under the cells in the adhesion zone represented by the parameter $\alpha$. The broken arrow shows the transcellular current flow at high frequencies $>10 \mathrm{kHz}$ mirrored by the capacitance $C_{m}$. C: Simplified equivalent circuit diagram describing an electrode covered with a confluent cell monolayer. $R_{\text {bulk }}$ represents the ohmic resistance of the electrolyte and CPE (constant phase element) accounts the fractal impedance of the electrode-electrolyte interface.

ECIS measurements were carried out using 400.000 MDCKII cells seeded onto gold electrodes (250 $\mu \mathrm{m}$ in diameter) of 8W1E arrays (Ibidi, Martinsried, Germany) and stored in an incubator set to $37^{\circ} \mathrm{C}$ and $5 \% \mathrm{CO}_{2}$. After a time interval of $24 \mathrm{~h}$, sufficient for adhesion and spreading of the cells, the cell layer reached confluency. Time-resolved frequency scans (impedance spectra) were acquired with an ECIS Ztheta-setup (Applied Biophysics, Troy, NY). Frequency sweeps were subjected to complex fitting employing an area contact model of Lo and Ferrier [129], resulting in time resolved parameters $R_{b}$ reflecting the transepithelial resistance, $\alpha$ being related to the cell-substrate distance ( $\alpha \propto \mathrm{h}^{-}$ $\left.{ }^{1 / 2}\right)$, and $C_{m}$ mirroring the membrane capacitance of the cells $\left(C_{m} \propto \mathrm{A}\right)$. 


\subsection{Cell Culture}

MDCK II cells, obtained from the Health Protection Agency, Salisbury, UK were maintained in minimal essential medium (MEM) with Earle's salts and $2.2 \mathrm{~g} / \mathrm{L} \mathrm{NaHCO}_{3}$ (Biochrom, Berlin, Germany) supplemented with $4 \mathrm{mM}$ L-glutamine Glutamin (Biochrom, Berlin, Germany), and 10\% FCS (PAA, Pasching, Austria) at $37^{\circ} \mathrm{C}$ in a $5 \% \mathrm{CO}_{2}$ humidified incubator. Medium for samples which are prepared or measured outside the laminar flow box contains additionally penicillin (0.2 $\mathrm{mg} \mathrm{mL}^{-1}$, PAA, Pasching, Austria), streptomycin (0.2 mg mL ${ }^{-1}$, PAA, Pasching, Austria), und amphotericin B (5 $\mu \mathrm{g} \mathrm{mL} \mathrm{m}^{-1}$, PAA, Pasching, Austria). HEPES is supplemented in a concentration of $15 \mu \mathrm{M}$ (PAA, Pasching, Austria). Confluent cells were released with trypsin/EDTA (0.5\% / $0.2 \%)$ (Biochrom, Berlin, Germany) and subcultured weekly. For spreading studies cells were trypsinized for 5 min and used within 30 min after detachment. 


\section{Results and Discussion}

\subsection{Mechanical Properties of confluent MDCK II Cells ${ }^{2}$}

The goal of this study is to investigate how polar epithelial cells adjust their surface area in response to tension changes induced by external stimuli that modulate cytoskeletonmembrane attachment, cytoskeleton integrity, cholesterol content, and morphology of the apical membrane. The complex interplay between cytoskeleton and plasma membrane requires a well-established model system with limited perturbation from cell growth, migration, and proliferation. Here, confluent Madin-Darby canine kidney cells (MDCK II) are used to investigate tension-controlled cellular area regulation. Indentation experiments combined with pulling of membrane tethers carried out with an atomic force microscope (AFM) are used to assess local changes in membrane tension and excess membrane area as a function of external stimuli. This approach allows, for the first time, to simultaneously measure membrane tension and surface area at the same spot proving unprecedented insight in the mechanical interplay of tension and area regulation. First, the focus lies on the mechanical properties of untreated cells and how the surface structure contributes to it.

\subsubsection{Structural Characterization of MDCK II Cells}

MDCK II cells establish under culture conditions confluent monolayers. In such a monolayer, the cells are tightly packed and connected by cell-cell contacts (Figure $32 \mathrm{~A}$ and Figure $41 \mathrm{E}-\mathrm{H})$. From AFM images the cellular morphology is estimated, giving an average radius of $R_{c}=10 \mu \mathrm{m} \pm 0.8 \mu \mathrm{m}$ and a cell height of $h=1 \mu \mathrm{m} \pm 0.2 \mu \mathrm{m}$. The cellular surface exhibits a highly roughened structure (Figure $32 \mathrm{~A}, \mathrm{D})$.
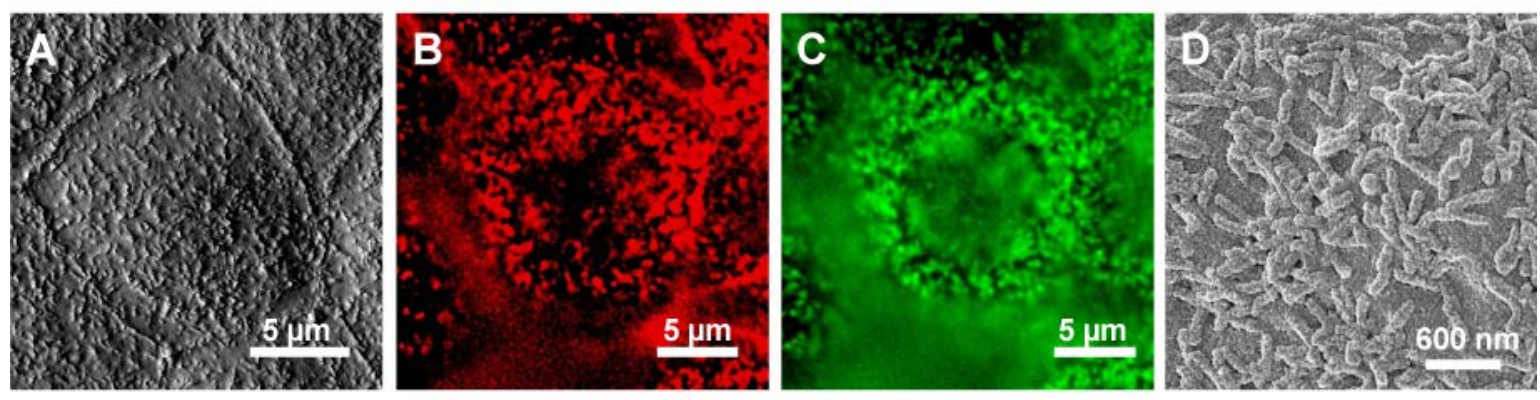

\footnotetext{
${ }^{2}$ Parts of this chapter are published in

Pietuch, A.; Brückner, B.R.; Fine, T.; Mey.I., Janshoff, A. (2013) Elastic properties of cells in the context of confluent cell monolayers: impact of tension and surface area regulation. Soft Matter, 2013, 9 (48), 11490 11502.
} 
Figure 32: Surface characterization of confluent MDCK II cells. A: AFM deflection image. B: Fluorescence micrograph of F-actin and (C) ezrin staining, demonstrating co-localized structures in the apical part of the cell. D: SEM image of the surface of MDCK II cells. Clearly visible are membrane protrusions.

The structured surface is apparent in AFM and SEM images. Besides, the intracellular organization underlying the membrane is established by an accumulation of F-actin and ezrin. The membrane protrusions are stabilized by bundled F-actin anchored to the membrane by the protein ezrin (Figure $32 \mathrm{~B}, \mathrm{C}$ ). This cortical arrangement gives the cellular membrane its shape. The cortex is a dynamic structure subjected to rapid remodeling as induced by processes like cell-division (Figure 33).
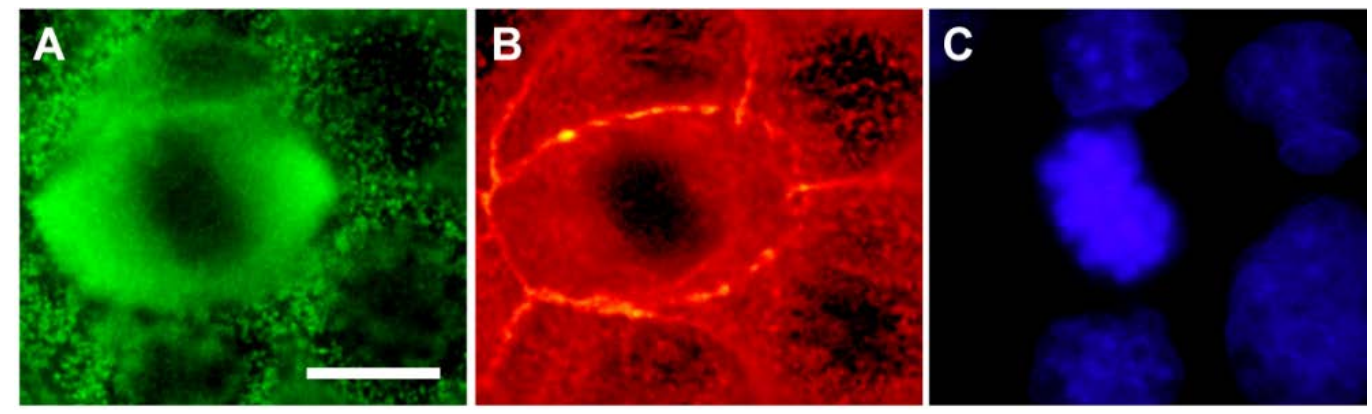

Figure 33: Fluorescence micrographs of MDCK II cells showing changes in cortical structure during cell division. A: Ezrin staining. B: F-actin. C: Cell nucleus. Scale bar is $10 \mu \mathrm{m}$.

Figure 33 shows how the protein distribution changes at the plasma membrane during cell division. Both F-actin and ezrin reorganizes. The typical dotted structure of F-actin in the cellular apex disappeared. It is predominant found in the cell periphery at the cell-cell contacts. Ezrin is diffusely distributed. This ability of reorganization gives the plasma membrane the opportunity to change the shape in response to shape changes upon division, cell growth and spreading. Since the cellular plasma membrane reveals these dynamic and adaptable properties, a precise mechanical investigation is of high interest. First the spatiotemporal mechanics of confluent and untreated MDCK II cells are under investigation. In the following sections, the plasma membrane is exposed to different substances, targeting the cytoskeleton integrity, the attachment between plasma membrane and cytoskeleton, changing its mechanical characteristics.

\subsubsection{Mechanical Measurements}

The mechanical properties of the plasma membrane of confluent MDCK II cells, is examined by indentation and tether pulling experiments, as described in section 3 and 4 . 
Force distance curves obtained from force mapping are analyzed by applying the Hertz model treating cells as elastic continua. In order to place these experiments in a mechanical context the Hertzian contact model is used. This reveals local mechanical properties of the cells (Figure 34). Cell boundaries display a more rigid structure, as they appear lighter in the Young's modulus map. The center of the cells shows a uniformly distributed elasticity. It is noteworthy that the cellular nucleus is not visible in the Young's modulus map, although they are observable in the AFM height image as higher (lighter) regions, as well as in phase contrast microscopy (Figure $34 \mathrm{~A}, \mathrm{C}$ ). This observation indicates that here indentation experiments measure the mechanical properties of the cellular cortex rather than the mechanical contribution from the whole cell interior, including the nucleus.
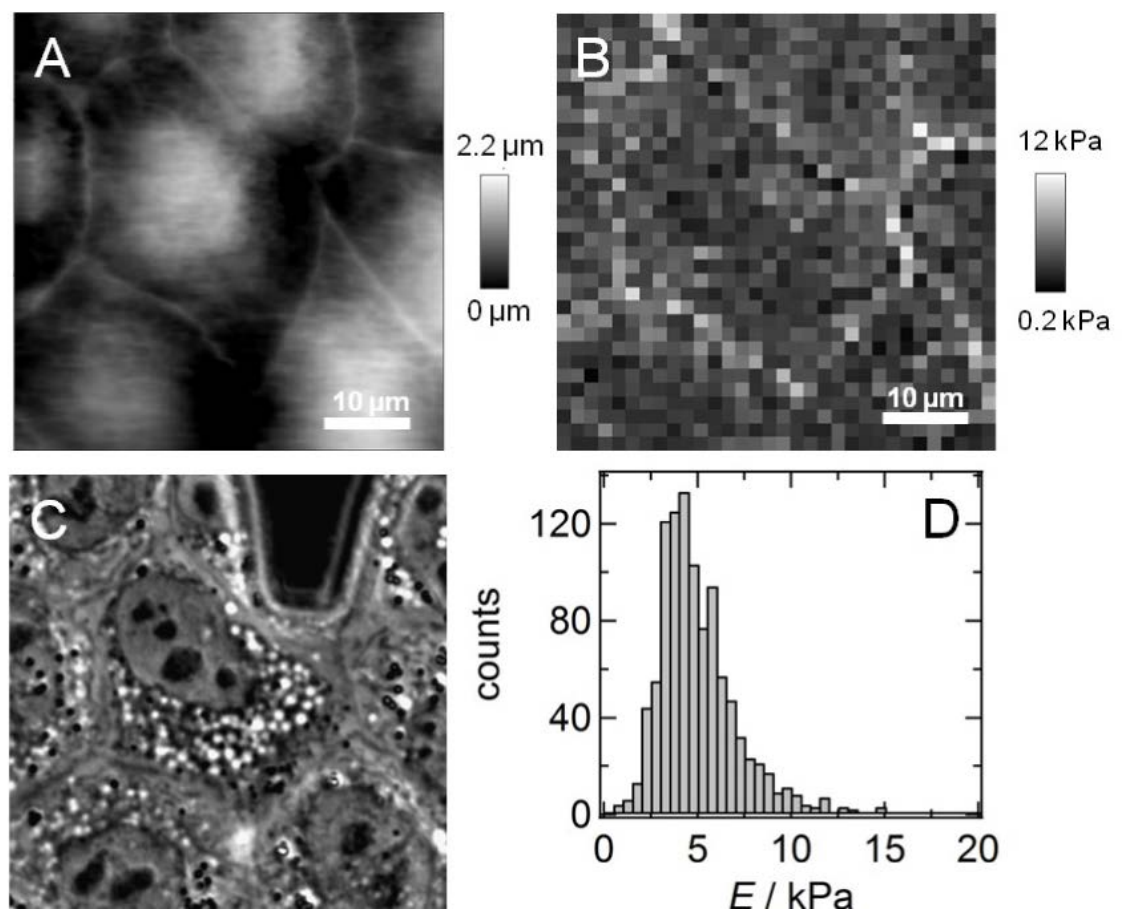

Figure 34: Mechanical investigation of confluent MDCK II cells. A: AFM height image. B: Young's modulus map obtained from fitting the model to force distance curves as described in section 3.1. C: Phase contrast micrograph of the same position as in A and B. In the upper part of the image the cantilever is visible. D: Histogram of Young's modules obtained from force map in B.

The average Young's modulus of confluent MDCK II cells is $(4.9 \pm 2.1) \mathrm{kPa}$. This is in good agreement with values found by others (Mathur et al.; 5-7 kPa [90]; Steltenkamp et al: $6.2 \pm 1.2 \mathrm{kPa}[130])$.

Although the Young's modulus characterizes the overall elasticity of a sample, the assumptions for using the Hertz-model do not reflect the cellular situation very well. 
Besides, when applying the model to the experimental data, only a small part of the force curve is considered (first $500 \mathrm{~nm}$ ), for reasons explained in section 3.1. Therefore, we used a different model by regarding the cell as an object with constant surface tension (liquid droplet).
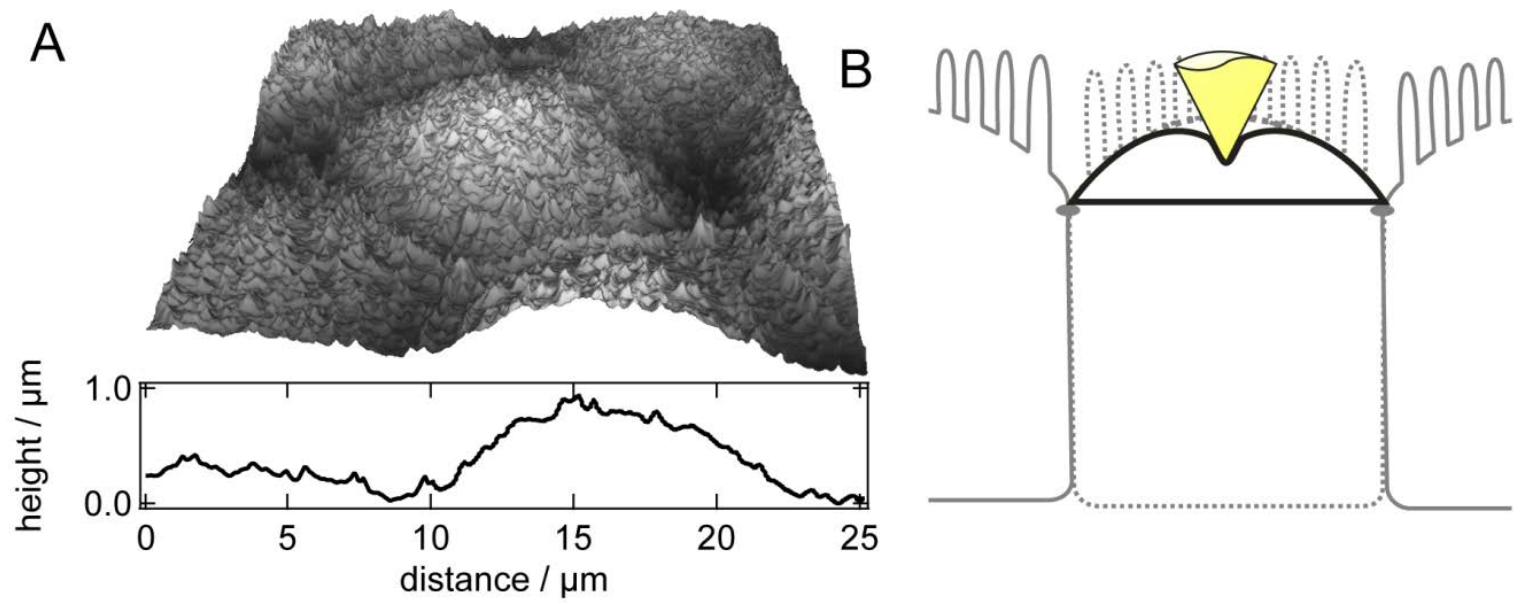

Figure 35: A: Three-dimensional AFM height image of confluent MDCK II cells. Line scan represents the first line in the 3D image. The apical membrane is substantially roughened due to the presence of microvilli. B: Schematic drawing illustrating modeling of MDCK II cells as spherical caps limited by cell-cell contacts.

We assume that the cell-cell contacts connected to the contractile actin ring serve as tension-generating boundaries justified by AFM topography images of confluent MDCK II cells.

An extended liquid-droplet model is adopted to the data, as described by Discher and coworkers to quantify the indentation of the apical cap, which essentially assumes a lateral tension $T$ composed of a lateral tension or pre-stress $T_{0}$ governing the response to indentation at low penetration depth and non-linear in-plane stretching of the shell characterized by a 2-D Hookean term [25]. The apparent area compressibility modulus $\tilde{K}_{A}$ depends on the excess membrane area $A_{e x}$ and the area compressibility of the plasma membrane $K_{A}$. A large excess of surface area generates smaller apparent area compressibility modules (Equation 9, 19). Concomitant to indentation experiments, membrane tension is measured by means of tether pulling, as described in section 3.3. 

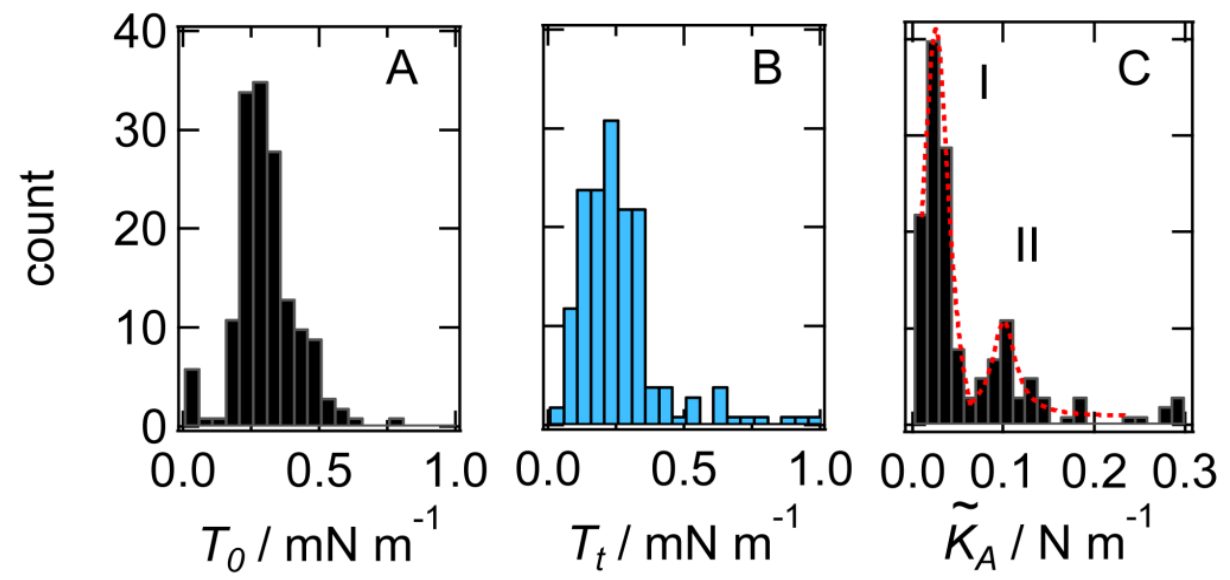

Figure 36: Results of force cycle measurements to assess the mechanical properties of the epithelial cell line MDCK II. A: Histogram of tension values $T_{0}$ obtained from indentation experiments $(n=160)$. B: Histogram of tension values $T_{t}$ calculated from tether pulling experiments using $(n=750)$. C: Histogram of apparent area compressibility module $\tilde{K}_{A}$ showing a bimodal distribution $(n=160)$. The dotted red line represents a Gaussian fit of peak I and II.

Figure 36 shows histograms of tensions $T_{0}, T_{t}$, and apparent area compressibility $\tilde{K}_{A}$ of confluent MDCK II cells. Interestingly, both tension values obtained from either pulling $\left(\bar{T}_{t}=0.29 \pm 0.17 \mathrm{mN} \mathrm{m}^{-1}\right)$ or indentation, $\left(\bar{T}_{0}=0.3 \pm 0.12 \mathrm{mN} \mathrm{m}^{-1}\right)$ are essentially identical (Figure 36). The apparent area compressibility module $\tilde{K}_{A}$ of untreated MDCK II cells shows a bimodal distribution, with one maximum centered at $\tilde{K}_{A}=0.02 \mathrm{~N} \mathrm{~m}^{-1}$ (68\%) and the second one at $\tilde{K}_{A}=0.1 \mathrm{~N} \mathrm{~m}^{-1}$ (peak II, $32 \%$ ). It is commonly accepted that $\tilde{K}_{A}$ values of pure lipid bilayers and cell membranes are in the range of $0.1-1 \mathrm{~N} \mathrm{~m}^{-1}$ depending on their composition [71, 131]. Significantly lower $\tilde{K}_{A}$-values are therefore indicative of excess membrane area stored in the highly roughened surface of the apical membrane encompassing protrusions such as microvilli and invaginations like caveolae. This excess area or membrane folding of MDCK cells was quantified by Butor and Davoust. They found that MDCK II cells display a folding factor of $\sim 8$ for the apical plasma membrane [132], which is in good agreement with the findings here (folding factor of $\sim 5$ following Equation 19).

Structural organization and stabilization of the plasma membrane protrusions relies heavily on protein linkers such as ezrin connecting the cellular membrane to microfilaments [11, 15]. 

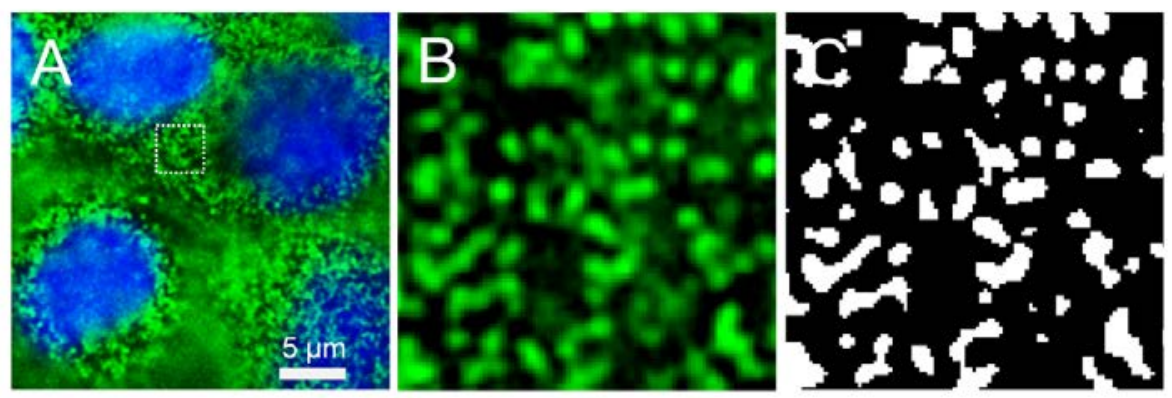

Figure 37: Analysis of fluorescence micrographs of cellular apex of MDCK II cells. (A) Cells are labeled against ezrin (green) and nucleus (blue). (B) Region of interest from micrograph (A) (100x100 pixel). Green fluorescence displays the protein ezrin. (C) White pixels correspond to green staining in (B). Analysis shows a distribution of white to black pixels of $32 \%$ to $68 \%$.

Fluorescence micrographs of ezrin-labeled MDCK II cells are analyzed, displaying protein coverage of $32 \%$ on the cellular apex, mirroring also quantitatively the bimodal distribution of the $\tilde{K}_{A}$ values (Figure 37). This emphasize, that the distribution reflects two distinct topographical regions on the apical cell membrane.

Here, tether pulling is used as second mechanical approach to determine the membrane tension. Since the membrane tension $T_{0}$ found by indentation experiments matches the tension from tether pulling of confluent MDCK II cells, it is assumed that the liquid droplet model applies to the cell type under investigation. But until now the viscous contribution to the tether force (described in section 3.3) is neglected. In order to assess the viscous contribution to the membrane tension $T_{t}$ (Eq. 32), membrane viscosity $\eta$ is estimated in the following section.

\subsubsection{Membrane Viscosity}

To investigate the viscous contribution to the tether forces, membrane nanotubes are pulled from the plasma membrane of MDCK II cells at different velocities (2, 4, 8, 16 and $20 \mu \mathrm{m}$ $\left.\mathrm{s}^{-1}\right)$. Equation 32 suggests a linear relationship between tether force and pulling velocity. The same is found here (Figure 38).

From the obtained linear equation and Equation (32) the membrane viscosity $\eta$ is calculated $\eta=\left(0.89 \cdot 10^{-6} \pm 0.18 \cdot 10^{-6}\right) \mathrm{Ns} \mathrm{m}^{-1}$. 


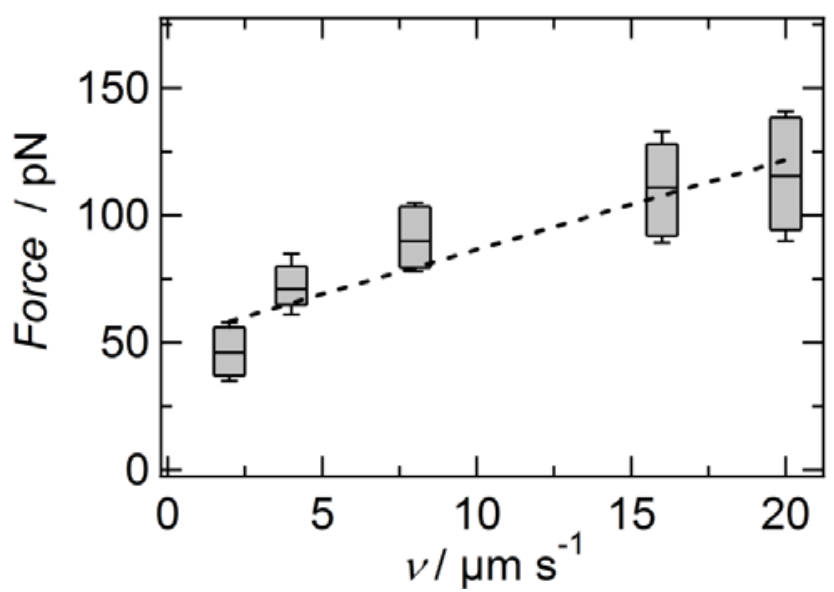

Figure 38: Tether force represented as boxplots plotted against different pulling velocities. Dashed line represent linear fit giving the linear equation $F=51.4 \cdot 10^{-11} \mathrm{~N}+3.53 \cdot 10^{-6} \mathrm{Ns} \mathrm{m}-1 \cdot v$.

The viscous contribution (second term of Equation 32) to the tether force at $v=2 \mu \mathrm{m} \mathrm{s}^{-1}$, is estimated to be $F_{\text {tether, viscous }}=7.1 \mathrm{pN}$. Calculating the consequential deviation in membrane tension leads to an increase of the values of $T_{t}$ by $0.006 \mathrm{mN} \mathrm{m}^{-1}$, which is small and in the range of the stated error of $T_{t}\left(0.29 \pm 0.17 \mathrm{mN} \mathrm{m}^{-1}\right)$. Therefore, viscous contributions to the tether force can be neglected.

\subsubsection{Bending Rigidity and Tether Radius}

From tether pulling experiment, indentation experiments and Equation 32 it is also possible to estimate the bending rigidity $\kappa$ of the plasma membrane of MDCK II cells (vide infra). Parameters used to calculate $\kappa$ are listed in Table 6:

Table 6: Mechanical parameter for calculating the bending rigidity $\kappa . T_{0}$ is obtained from indentation experiments.

\begin{tabular}{cccc}
\hline $\begin{array}{c}T_{0}\left[\mathrm{mN} \mathrm{m}^{-1}\right] \\
n=160\end{array}$ & $\begin{array}{c}F_{\text {tether }}[\mathrm{pN}] \\
n=844\end{array}$ & $\eta\left[\mathrm{Ns} \mathrm{m}^{-1}\right]$ & $v\left[\mu \mathrm{m} \mathrm{s}^{-1}\right]$ \\
\hline $0.3 \pm 0.12$ & $47 \pm 16$ & $0.89 \cdot 10^{-6} \pm 0.18 \cdot 10^{-6}$ & 2
\end{tabular}

Solving Equation 32 for $\kappa$ gives:

$\kappa=\frac{F_{t e t h e r}{ }^{2}}{8 \pi^{2} T_{0}}-\frac{\eta^{2} v^{2}}{C^{2} 2 T_{0}}$,

Using values from table 6 a bending rigidity of $1.02 \cdot 10^{-19} \pm 0.65 \cdot 10^{-19} \mathrm{Nm}$ is found, corresponding well with the bending modulus of a neat lipid bilayer. A value of $1 \cdot 10^{-19} \mathrm{Nm}$ is reported for a fluid lipid bilayer [27]. This finding proves the consistency of the applied 
liquid droplet model in which the restoring force to indentation is assumed to be generated solely by the tensed plasma membrane.

From the in-plane tension $T_{t}$ and the bending rigidity $\kappa$ of the plasma membrane of MDCK II cells, the radius of a pulled tether is determined according to Equation (33), yielding $R_{t}=$ $12 \mathrm{~nm} \pm 4 \mathrm{~nm}$.

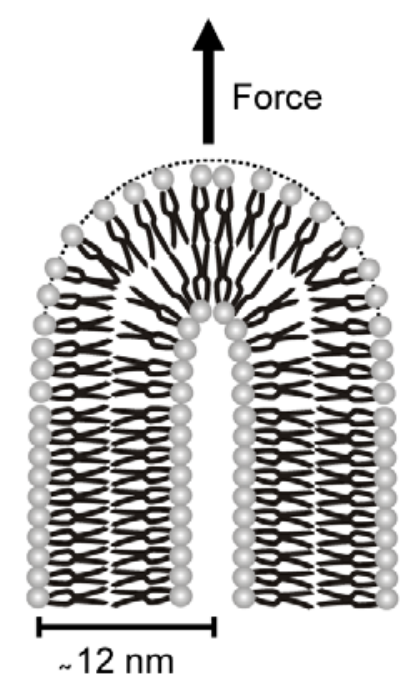

Figure 39: Schematic illustration of a pulled membrane nanotube.

This value is in good agreement with other reported tether radii ([107] [103] [133]). Interestingly, the tether radius here is similar to the radius found for small unilamellar vesicles (SUV) [134].

\subsubsection{Discussion}

The mechanical investigation of confluent MDCK II cells reveals a Young's modulus of $4.9 \pm 2.1 \mathrm{kPa}$, which corresponds well to values found in literature [130] [90]. The Hertzian mechanical model assumes, even for cellular mechanics, sample properties to be homogenous and isotropic and linear elastic. For the most cell lines it is more appropriate to model the cell with a cortical surface tension rather than assuming a homogeneous elastic body characterized by a single Young's modulus, since the membrane underlying cortex exerts tension to the membrane. Treating the cells as liquid droplets, we found that both techniques, indentation and tether pulling, provide essentially identical values for the lateral tension of untreated cells $\left(\sim 0.3 \mathrm{mN} \mathrm{m}^{-1}\right)$, which assumes that the liquid droplet model applies to the cell type under investigation. Similar values were obtained from 
micropipette suction experiments of neutrophil membranes [106, 135]. This is a clear hint that the tension $T_{0}$ measured in indentation experiments is due to in-plane tension of the membrane shell, originating from the attachment of the plasma membrane to the cytoskeleton and adherens junctions and is not an elastic property of the actin cortex (terminal web). The area compressibility module $\tilde{K}_{A}$ of untreated MDCK II cells displays a bimodal distribution centering on a low (peak I, $0.02 \mathrm{~N} \mathrm{~m}^{-1}$ ) and a high (peak II, $0.1 \mathrm{~N} \mathrm{~m}^{-}$ $\left.{ }^{1}\right) \tilde{K}_{A}$ value, respectively. We found some evidence that this distribution reflects two distinct topographical regions on the apical cell membrane. One region is associated with a substantial membrane reservoir giving rise to a low $\tilde{K}_{A}$ and a second population, in which the membrane constitutes a thin stretched shell that shows an area compressibility resembling those of artificial lipid bilayers or membranes from red blood cells [131, 136, 137]. Pre-stress giving rise to lateral tension in these regions is assumed to originate mainly from membrane attached to the actin network via ERM proteins as shown by immunostaining of ezrin and co-localization with actin filaments.

The membrane viscosity of the plasma membrane of MDCK II cells, determined by tether pulling experiments at different velocities, yield a value of $\eta=0.89 \cdot 10^{-6} \pm 0.18 \cdot 10^{-6} \mathrm{Ns} \mathrm{m}^{-}$ ${ }^{1}$. This is in good agreement with viscosities found by Sun et al. where they report a effective surface viscosity $\eta_{\text {eff }}$ of $0.15 \pm 0.03 \cdot 10^{-6} \mathrm{Ns} \mathrm{m}^{-1}$ for Bovine aortic endothelial cells (BAECs) [112]. Pure lipid bilayer exhibit a surface viscosity of $10^{-8}-10^{-6} \mathrm{Ns} \mathrm{m}^{-1}$ [138] [139]. For the calculation of the membrane tension from tether pulling experiments a bending rigidity of $1 \cdot 10^{-19} \mathrm{Nm}$ (fluid lipid bilayer) is assumed [27]. From the velocity depended tether pulling and indentation experiments a $\kappa$-value of $1.02 \cdot 10^{-19} \pm 0.65 \cdot 10^{-19}$ $\mathrm{Nm}$ is obtained. It can therefore be concluded that the plasma membrane of MDCK II cells displays the same elastic properties as a neat lipid bilayer. Moreover, the calculated radius of formed tether $\left(R_{t}=12.4 \mathrm{~nm} \pm 3.9 \mathrm{~nm}\right)$, reveals a reasonable value as tethers can be regarded as a cylindrical vesicle as illustrated in Figure 39. The smallest attainable size for a unilamellar vesicle is found to be $12 \mathrm{~nm}$ [134]. These observations show that the membrane nanotubes pulled out of a native plasma membrane of MDCK II cells lie in the range of small vesicles also found in vivo [140], displaying the importance of the mechanical properties of membrane. Furthermore, it should be noted that the radius of pulled tether is too small to contain any actin filaments. 


\subsection{Depletion of Membrane Reservoirs ${ }^{3}$}

If the low values of the apparent area compressibility module $\tilde{K}_{A} \quad\left(0.02 \mathrm{~N} \mathrm{~m}^{-1}\right)$ were indeed a result of excess area stored in membrane reservoirs such as microvilli or caveolae, removal of the membrane reservoir should lead to an increase in $\tilde{K}_{A}$ up to $K_{A}$ of a bilayer in contact to a cortex. In turn, membrane tension is eventually expected to increase due to lack of membrane reservoir acting as a buffer. To verify these assumptions, we added methyl- $\beta$-cyclodextrin (MBCD), which is known to flatten the apical membrane by reducing cholesterol from the plasma membrane. $80-90 \%$ of total cellular cholesterol can be efficiently extracted [141] from the plasma membranes with MBCD concentrations of 5-10 $\mathrm{mM}$ and exposure times of more than 2h [142-144].

\subsubsection{Morphological Alteration During Cholesterol Depletion}

Electric cell-substrate impedance sensing, allows to study various cell parameters simultaneously and also permits to measure partly inaccessible features of cell morphology such as the cell-substrate distance with high time resolution $(\Delta t \leq 1 \mathrm{~s})$.
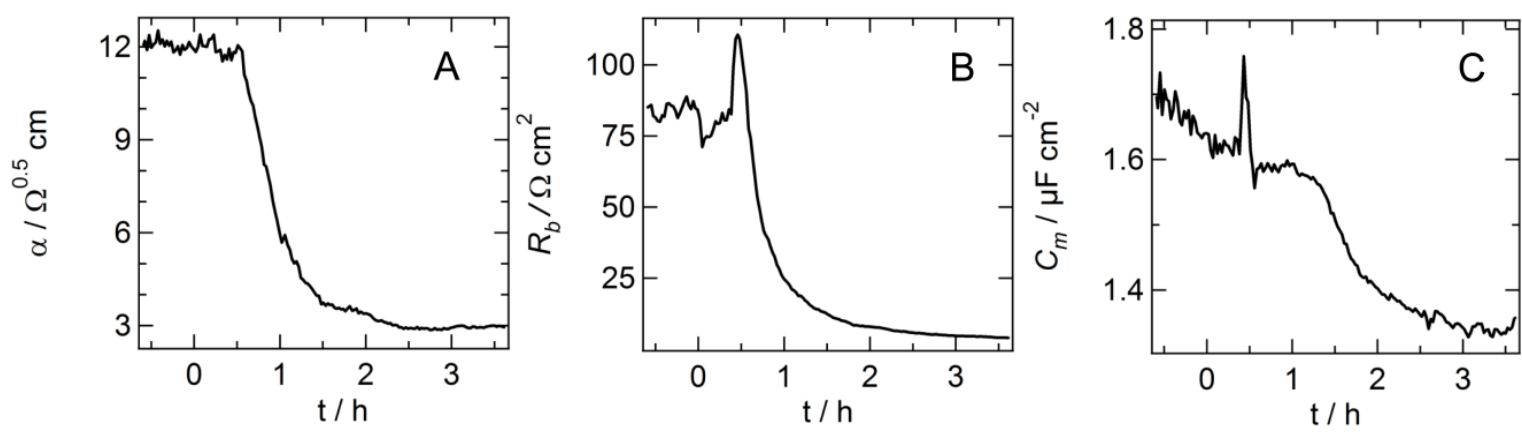

Figure 40: Time resolved ECIS measurement of MDCK II cell treated with MBCD analyzed by applying model based on Lo and Ferrier [129]. A: Parameter $\alpha$ describing current passing paracellular space decreases synchronously with $R_{b}$. B: Barrier resistance $R_{b}$ decreases after addition of MBCD to a final concentration of $10 \mathrm{mM}$. C: Specific membrane capacity $C_{m}$ shows a retarded declining effect after cholesterol depletion. Time point $0 \mathrm{~h}$ indicates MBCD addition.

Monitoring the cell specific parameter $R_{b}, \alpha$ and $C_{m}$ allows to quantify and categorize the impact of MBCD on the morphology of MDCK II cells (Figure 40). Depletion of cholesterol in the plasma membrane of MDCK II cells results in rapid drop in $\alpha$ from 12

\footnotetext{
${ }^{3}$ Parts of this chapter are published in

Pietuch, A.; Brückner, B.R.; Fine, T.; Mey.I., Janshoff, A. (2013) Elastic properties of cells in the context of confluent cell monolayers: impact of tension and surface area regulation. Soft Matter, 2013, 9 (48), 11490 11502.
} 
$\Omega^{0.5} \mathrm{~cm}$ to $3 \Omega^{0.5} \mathrm{~cm}$ for 3 hour of treatment with $10 \mathrm{mM}$ MBCD (Figure $40 \mathrm{~B}$ ). $\alpha$ is defined as $\alpha=0.5 W(\rho / h)^{1 / 2}$, where $W$ is cell width or radius, $\rho$ the specific extracellular medium resistivity, and $h$ is the average cell-substrate separation of the cell monolayer [145]. A decrease in $\alpha$ therefore reflects cell lifting up from the electrode. An enlargement of the gap between cell and substrate was observed in a static way by Francis et al with transmission electron microscopy [146]. To visualize the changes in the cell-substrate adhesion properties, the protein integrin is labeled during MBCD exposure (Figure $41 \mathrm{~A}$ D). Cellular binding to the substrate is due to focal contacts mediated by integrin clustering [147]. During the depletion of cholesterol, a change in the distribution of the protein integrin is observable at the basal part of the cell. After 3h of incubation integrins are localized at the cell periphery (Figure $41 \mathrm{D}$ ). Besides, integrins are also found in different focus planes, yielding the diffuse green appearance of the cell compared to untreated cells (Figure 41 A). This structural alteration in the cell-adhesion properties could have influence on the parameter $\alpha$.
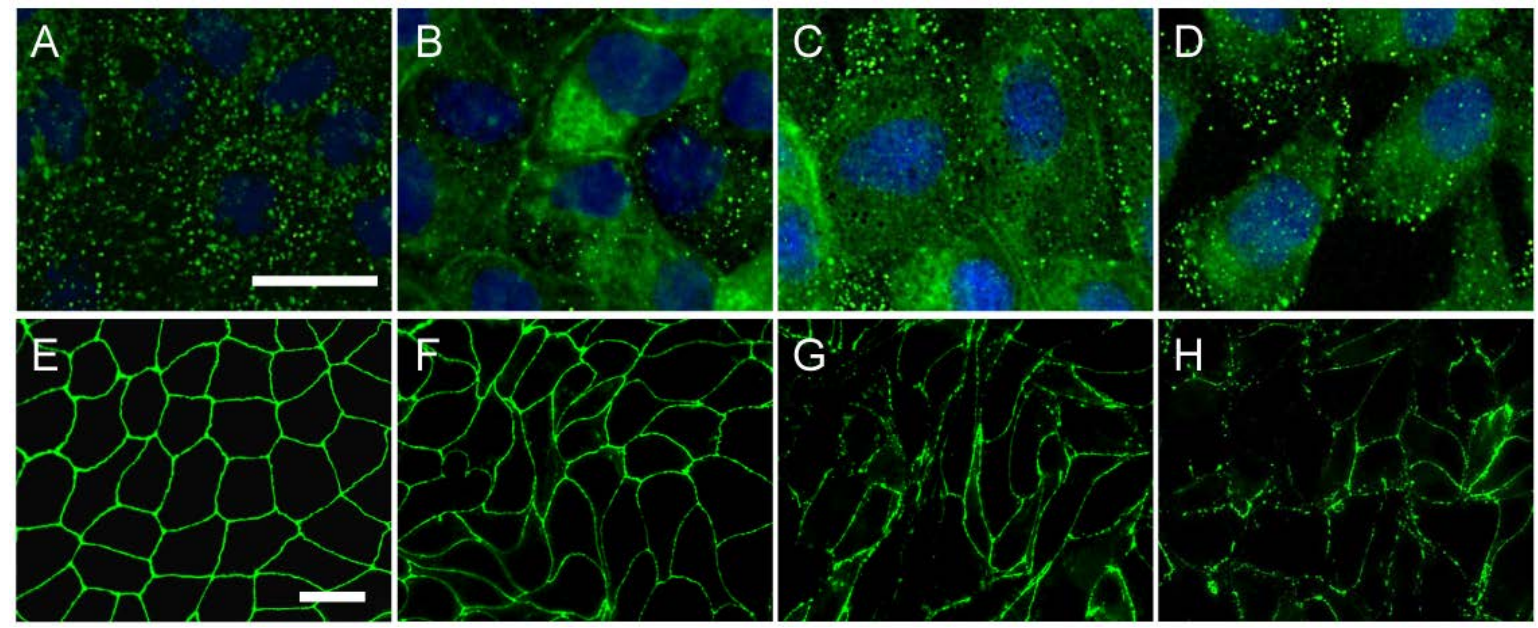

Figure 41: Fluorescence micrographs of MDCK II cells. In green the cell-substrate adhesion protein integrin is shown (A-D) and tight junction protein ZO-1 (E-F) after different exposure times to MBCD. A and E 0h, B and F $1 \mathrm{~h}, \mathrm{C}$ and $\mathrm{G} 2 \mathrm{~h}, \mathrm{D}$ and $\mathrm{H}$ 3h. Scal bar $10 \mu \mathrm{m}$.

Simultaneously, a reduction of the barrier resistance $R_{b}$ is found (Figure $40 \mathrm{~A}$ ). After one hour of exposure $R_{b}$ decreases from $80 \Omega \mathrm{cm}^{2}$ to values of $25 \Omega \mathrm{cm}^{2}$ and declines to a minimum of $4.5 \Omega \mathrm{cm}^{2}$ after 3 hours. This decrease depicts a change in the density of cellcell contacts, which is also demonstrated by immunofluorescence microscopy. Therefore, the tight-junction protein ZO-1 is stained. With progressing cholesterol extraction the dissolution of the cell-cell contacts is visible (Figure $41 \mathrm{E}-\mathrm{H}$ ). 
Interestingly, there is significant retardation in the impact of MBCD on the membrane capacity $C_{m}$ (Figure $40 \mathrm{C}$ ). One hour after adding the cholesterol scavenger to the confluent monolayer no changes in $C_{m}\left(1.6 \mu \mathrm{F} / \mathrm{cm}^{2}\right)$ are observable. The decrease in $C_{m}$ starts 1.3 hour after exposure to MBCD and a reaches minimum value of $1.3 \mu \mathrm{F} / \mathrm{cm}^{2}$ after 3 hours. The retarded effect of cholesterol depletion on the properties of the plasma membrane is also visible in the fluorescence micrographs of the actin cytoskeleton and ezrin in the apical domain of MDCK II cells (Figure 42).

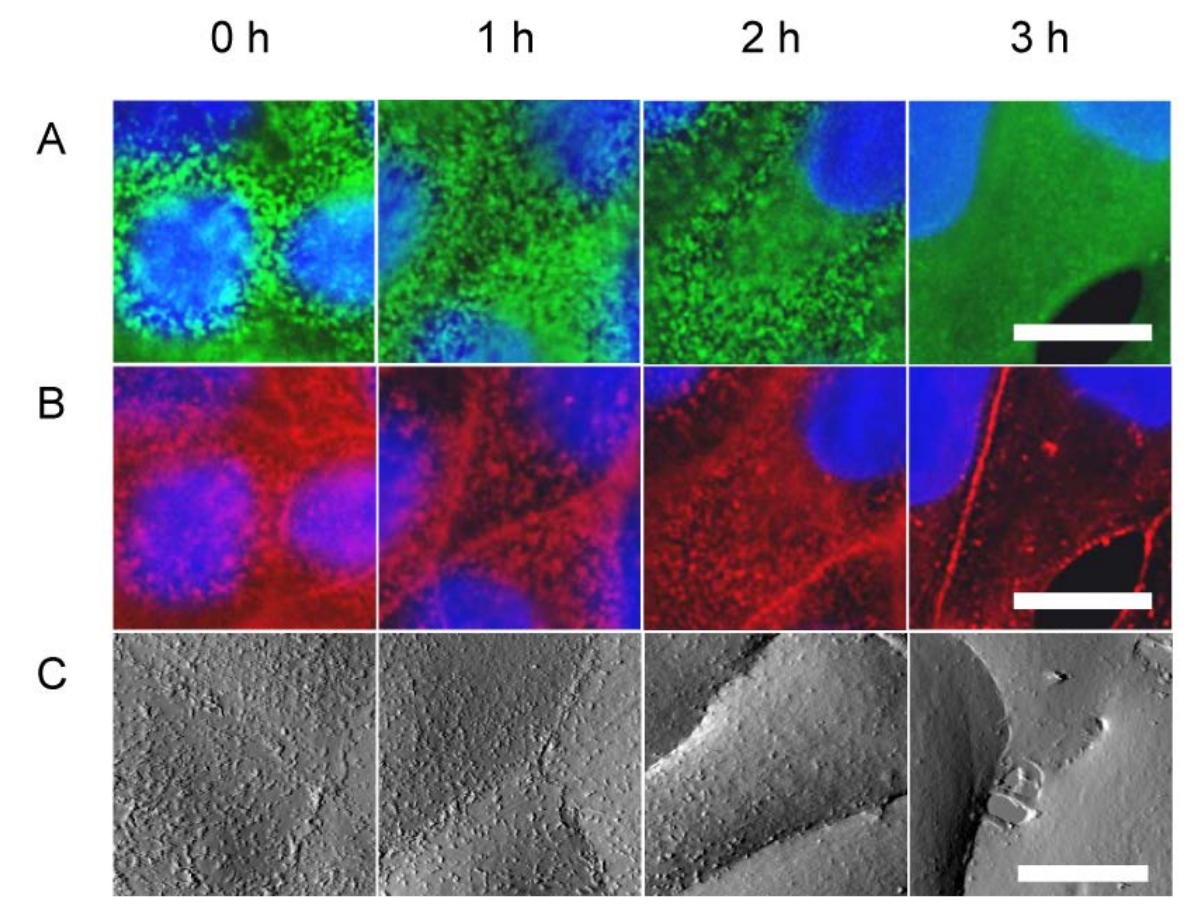

Figure 42: Effect of cholesterol depletion by MBCD, on the properties of the plasma membrane of MDCK II cells. Immunostaining of ezrin (green, A) and F-actin (red, B) and corresponding AFM deflection images (C), taken at different exposure times of MBCD. Untreated cells $(0 \mathrm{~h})$ display a large number of microvilli at the plasma membrane, which is reduced after $2 \mathrm{~h}$ of incubation with MBCD. After $3 \mathrm{~h}$ only few microvilli remain visible at the apical membrane of the cells. Scale bar: $10 \mu \mathrm{m}$.

Cholesterol depletion from the plasma membrane of MDCK II cells has great impact on the topological and molecular properties of the cellular membrane. Figure 42 shows, that the number of microvilli is greatly reduced after MBCD treatment. After 3h, immunostaining of ezrin and F-actin reveal a delocalizing of the proteins from the surface. The cell surface has lost almost all membrane protrusion and appears topographically smooth (Figure $42 \mathrm{C}$ ).

This structural alteration is also visible in SEM images (Figure 43), were the reduction in membrane protrusions and dissolution of cell-cell contacts is apparent. 


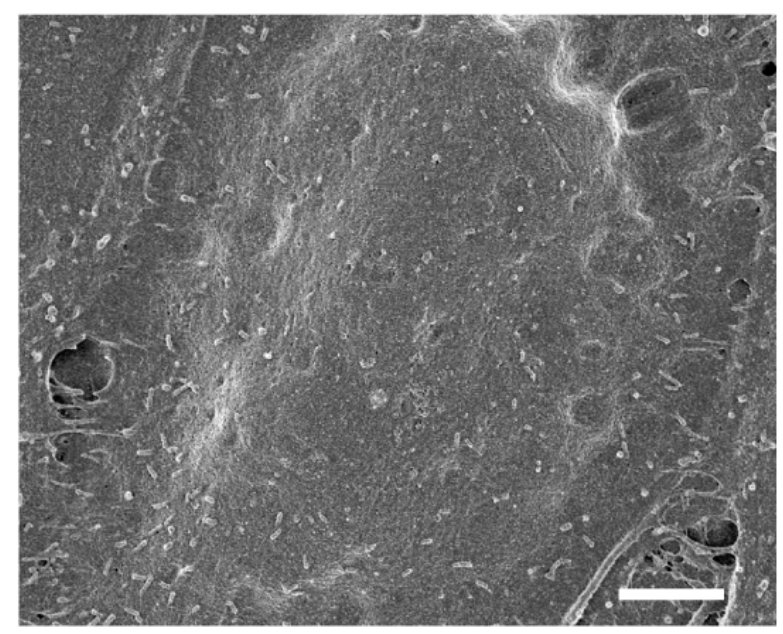

Figure 43: SEM image of MDCK II cells after exposure to cholesterol depleting MBCD for 3h. Scale bar 2 $\mu \mathrm{m}$.

A decrease in membrane capacitance $C_{m}$ is due to a reduction in the overall membrane area. To investigate this membrane loss the plasma membrane of MDCK II cells is labeled after the cells are exposed to MBCD.

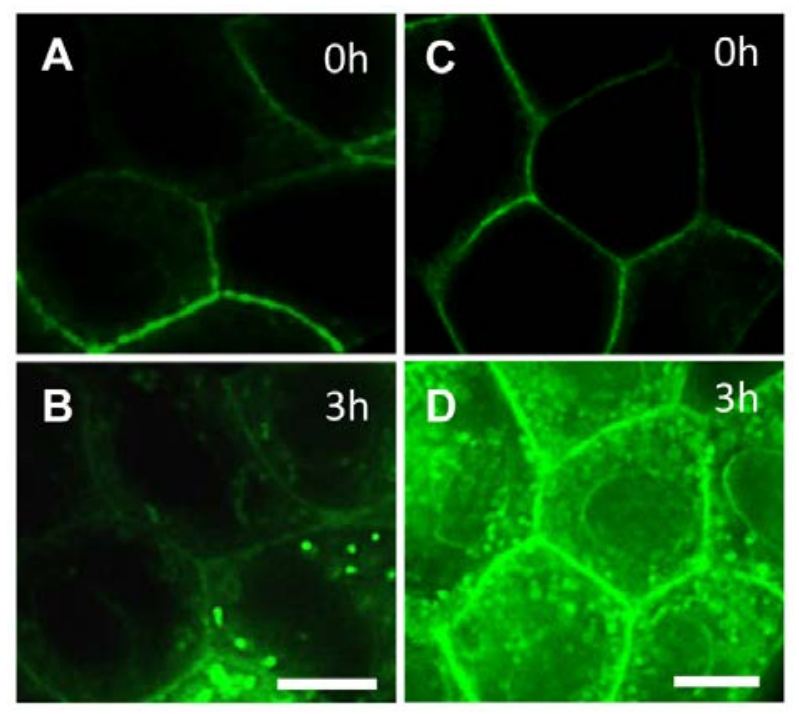

Figure 44: Membrane staining of living MDCK II cells to visualize membrane uptake after MBCD treatment. Focal plane is in the lower part of the cell (basal). A-B: Untreated cells to show normal endocytotic uptake rate. C-D Cells before and after $3 \mathrm{~h}$ treatment with MBCD. Green fluorescence displays membrane-uptake during cholesterol depletion, small vesicular structures are detectable in lower part of the cell (D).

Figure 44 shows a higher endocytosis rate as a response to cholesterol depletion, i.e. a larger number of vesicles accumulate in the cytosol.

The ECIS-method also allows the investigation of the reversibility of morphological changes imposed by cholesterol depletion. Therefore, medium containing $10 \mathrm{mM}$ MBCD is substituted with culture medium after a predetermined exposure time. All parameters $\alpha$, 
$R_{b}$ and $C_{m}$ return to initial values found for untreated cells displaying full reversibility of cholesterol depletion (Figure 45).
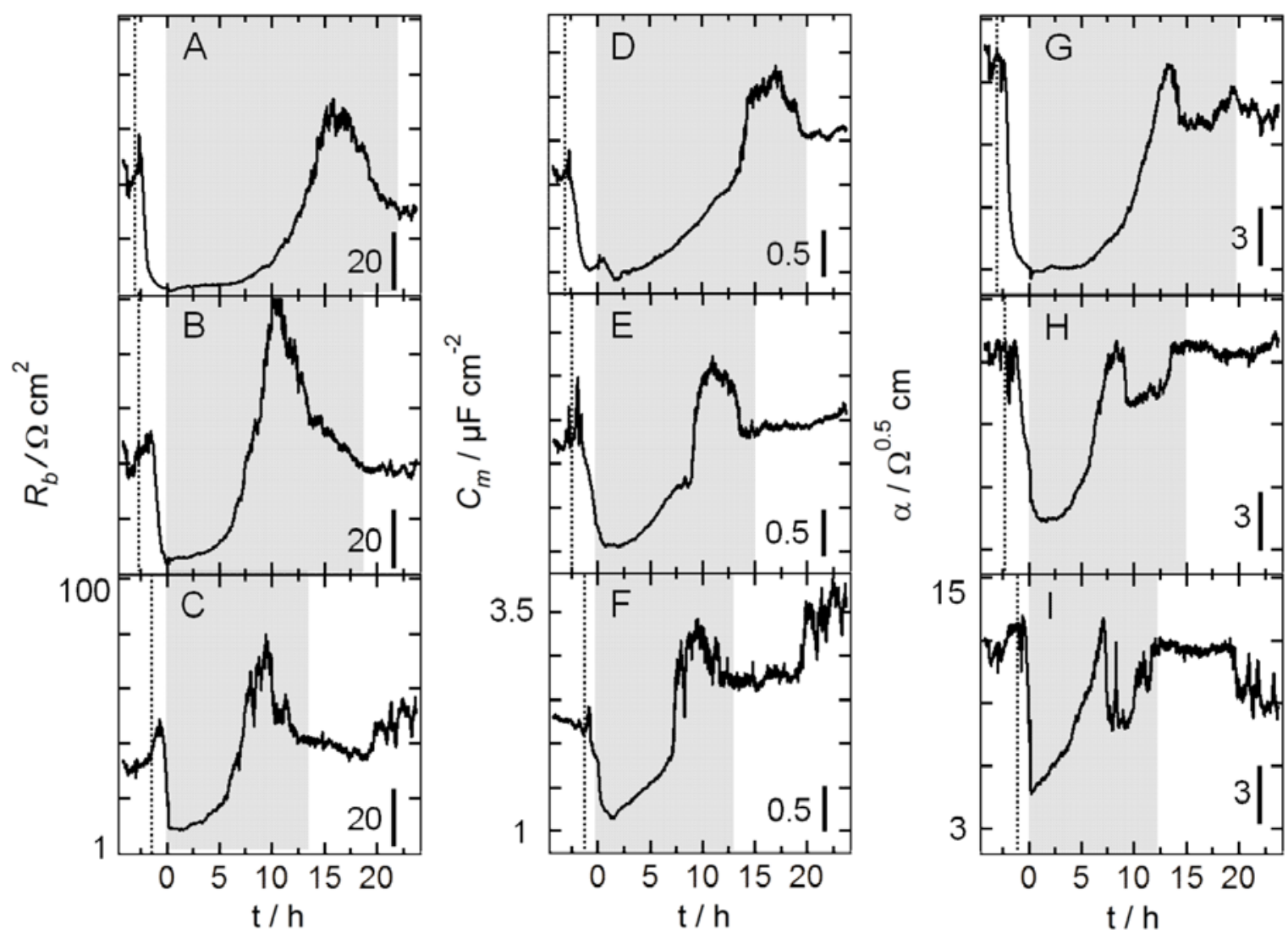

Figure 45: Parameter derived from ECIS measurements of MDCK II cells with different exposure times to MBCD (10 mM) and removal of MBCD after 1(lower row), 2 (middle row) and 3h (upper row). Dotted lines indicate point of substance addition. Time point 0 marks medium exchange. Grey area highlights the recovery period until the respective parameter regains its initial value prior to MBCD treatment.

The exchange of the medium after different exposure times leads to different recovery periods. Longer MBCD exposure results also in a longer recovery time, which can be explained by a more extensive cholesterol depletion which needs longer to be replenished. Incubating MDCK II cells for $3 \mathrm{~h}$, which is sufficient for decreasing $R_{b}, \alpha$ and $C_{m}$ to a minimum value $\left(2.3 \Omega \mathrm{cm}^{2}, 3.3 \Omega^{0.5} \mathrm{~cm}\right.$ and $\left.1.04 \mu \mathrm{F} / \mathrm{cm}^{2}\right)$, leads to a regeneration time of the cell-cell contacts, reflected by the barrier resistance, of $22 \mathrm{~h}$ (Figure 45A). After this time $R_{b}$ reaches the initial value of $30 \Omega \mathrm{cm}^{2}$ as found for untreated cells. In comparison, plasma membrane protrusion and organization of the basolateral membrane reform after $20 \mathrm{~h}$ to $C_{m}=2.6 \mu \mathrm{F} / \mathrm{cm}^{2}$ and $\alpha=11.9 \Omega^{0.5} \mathrm{~cm}$. By shortening the exposure time $(2 \mathrm{~h})$ the total reconstruction of the cell-cell contacts is achieved after $18 \mathrm{~h}$, whereas $\alpha$ and $C_{m}$ recovers already after 15 h (Figure 45C-D). Finally, a short exposure time of merely 1 h, which is seemingly not sufficient to fully reduce $R_{b}\left(10.8 \Omega \mathrm{cm}^{2}\right)$ and $C_{m}\left(1,45 \mu \mathrm{F} / \mathrm{cm}^{2}\right)$, 
show the fastest reformation of cell barriers (14 h), substrate adhesion zone (12 h) and cell surface structures $(10 \mathrm{~h})$. It should be noted that recovery of plasma membrane capacity occurs faster than rebuilding of cell-cell contacts, while degradation of microvilli is considerably slower that dissolution of cell-cell contacts.

Table 7: ECIS parameter from experiment shown in Figure 41.

\begin{tabular}{lcccc}
\hline & according Fig. 41 & before MBCD & after MBCD & recovery \\
\hline$R_{b} / \Omega \mathrm{cm}^{2}$ & A & $41+/-3.5$ & $2.3+/-0.3(3 \mathrm{~h})$ & $30+/-0.8(22 \mathrm{~h})$ \\
& B & $44+/-1.7$ & $6.5+/-2.5(2 \mathrm{~h})$ & $37+/-0.5(18 \mathrm{~h})$ \\
& C & $37+/-2.9$ & $11+/-4.2(1 \mathrm{~h})$ & $40+/-1.2(14 \mathrm{~h})$ \\
$C_{m} / \mu \mathrm{F} / \mathrm{cm}^{2}$ & D & $2.1+/-0.03$ & $1.0+/-0.02(3 \mathrm{~h})$ & $2.6+/-0.02(20 \mathrm{~h})$ \\
& E & $2.3+/-0.11$ & $1.5+/-0.17(2 \mathrm{~h})$ & $2.3+/-0.03(15 \mathrm{~h})$ \\
$\alpha / \Omega^{0.5} \mathrm{~cm}$ & F & $2.2+/-0.04$ & $1.4+/-0.19(1 \mathrm{~h})$ & $2.8+/-0.06(11 \mathrm{~h})$ \\
& G & $12.8+/-0.5$ & $3.3+/-0.1(3 \mathrm{~h})$ & $11.9+/-0.1(20 \mathrm{~h})$ \\
& H & $12.6+/-0.3$ & $7.9+/-0.7(2 \mathrm{~h})$ & $12.7+/-0.2(15 \mathrm{~h})$ \\
& I & $12.2+/-0.2$ & $5.4+/-1.2(1 \mathrm{~h})$ & $11.8+/-0.2(12 \mathrm{~h})$ \\
\hline
\end{tabular}

In conclusion, the depletion of cholesterol from the plasma membrane of MDCK II cells by methyl- $\beta$-cyclodextrin (MBCD) results in reversible loss of microvillar surface structures, cell-cell contacts and a reorganization of basolateral membrane. The decrease in membrane area due to reduction of membrane protrusions is related to the observed higher endocytosis rate. 


\subsubsection{Mechanical Changes Due to loss of Membrane Reservoir}

The impact of cholesterol depletion from the plasma membrane and therefore the decrease in membrane area is first examined with the Hertzian approach. The mechanical properties of the cells are monitored by force mapping (Figure 46).
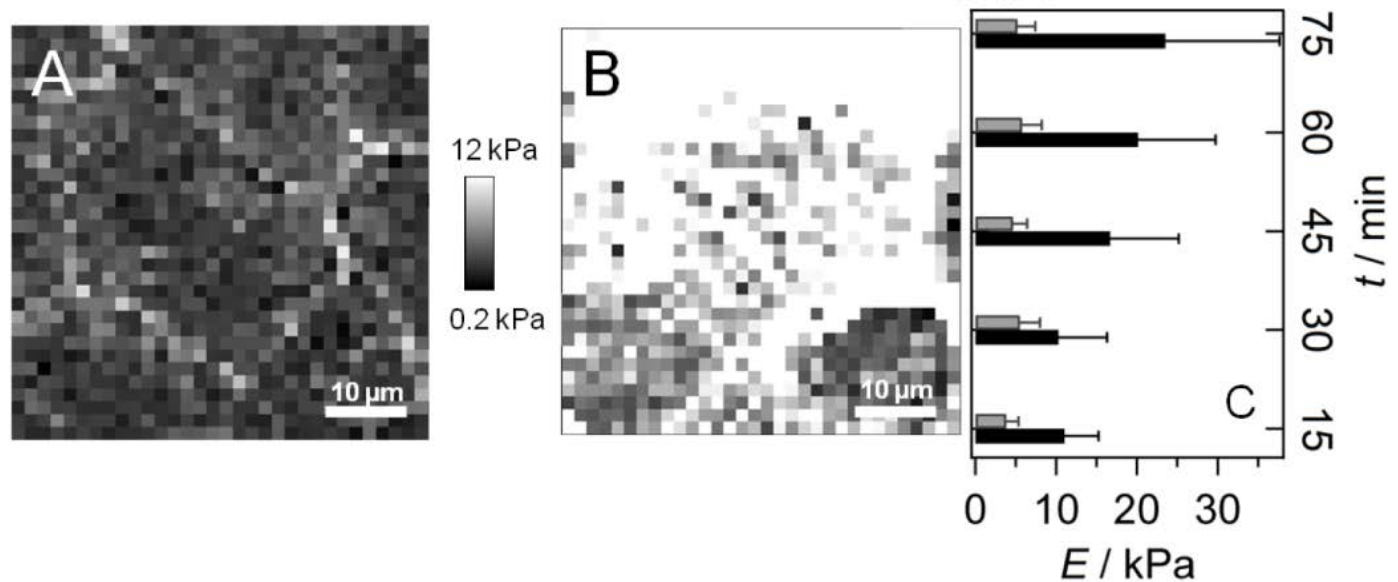

Figure 46: Changes of the Young's modulus of MDCK II cells during exposure to cholesterol depleting MBCD. A: Young's modulus map of confluent MDCK II cells before treatment. B: Young's modulus map during MBCD treatment. Force map starts in the lower left corner $15 \mathrm{~min}$ after substance addition. C: The Young's modulus increases with progressing cholesterol depletion. Gray bars display Young's modulus of untreated cells in A. Black bars show Young’s modulus during cholesterol depletion.

While the cholesterol is depleted and the overall plasma membrane area is decreased, the Young's modulus of the cells increases. Within the first 15 min of exposure to MBCD the cell stiffness increases from $3.9 \pm 1.5 \mathrm{kPa}$ to $11.1 \pm 4.2 \mathrm{kPa}$ (Table 8). After $75 \mathrm{~min}$ the Young's modulus rises to a value of $23.5 \pm 14.2 \mathrm{kPa}$.

Table 8: Young's modules of MDCK II cells during cholesterol depletion. For time point 15 min line 1 from force map in A or B is averaged $(n=32)$, etc. Data are presented as mean values with standard deviation.

\begin{tabular}{cccccc}
\hline $\begin{array}{c}\text { exposure } \\
\text { time }\end{array}$ & $\begin{array}{c}15 \mathrm{~min} \\
\text { (line 1) }\end{array}$ & $\begin{array}{c}30 \mathrm{~min} \\
\text { (line 8) }\end{array}$ & $\begin{array}{c}45 \mathrm{~min} \\
\text { (line 16) }\end{array}$ & $\begin{array}{c}60 \mathrm{~min} \\
\text { (line 24) }\end{array}$ & $\begin{array}{c}75 \mathrm{~min} \\
\text { (line 32) }\end{array}$ \\
\hline $\begin{array}{c}\mathrm{E}[\mathrm{kPa}] \\
\text { untreated }\end{array}$ & $3.9 \pm 1.5$ & $5.6 \pm 2.5$ & $4.7 \pm 1.7$ & $5.8 \pm 2.4$ & $5.2 \pm 2.2$ \\
$\begin{array}{c}\mathrm{E}[\mathrm{kPa}] \\
\text { treated }\end{array}$ & $11.1 \pm 4.2$ & $10.3 \pm 5.9$ & $16.7 \pm 8.5$ & $20.2 \pm 9.6$ & $23.5 \pm 14.2$ \\
\hline
\end{tabular}

The effect of cell stiffening after cholesterol depletion is also reported by Byfield and coworkers, where they show an increase in the Young's modulus of Bovine aortic endothelial cells (BAECs) by a factor of almost 2 [148]. 
Since the Hertzian approach suffers from many assumptions (details in section 3), the cholesterol depletion from the plasma membrane is examined with a second mechanical model, the liquid droplet. Force indentation curves were subject to nonlinear fitting of the model according to Discher and coworkers allowing to extract $T_{0}$ and $\tilde{K}_{A}$. Concomitant, membrane tether are pulled to confirm tension values from indentation experiments.
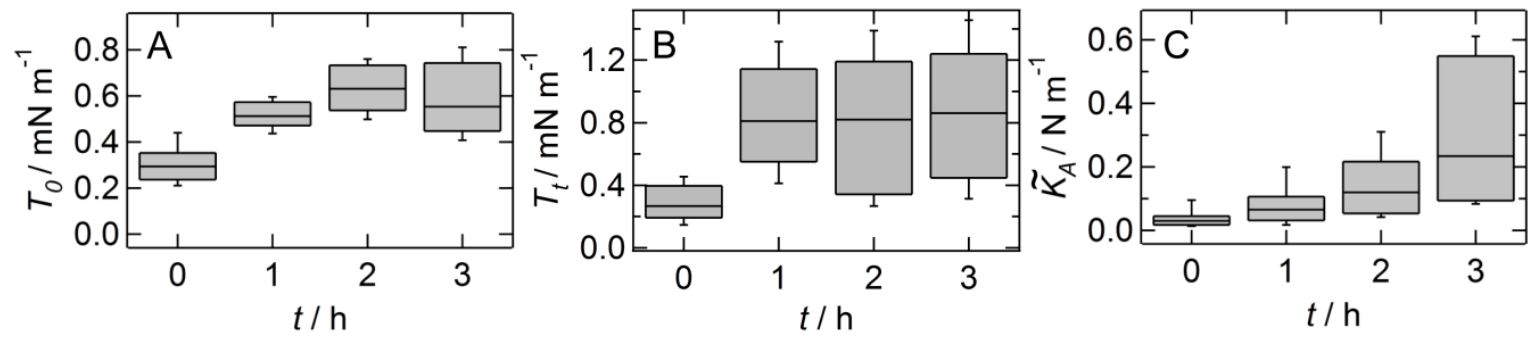

Figure 47: Effect of cholesterol depletion by MBCD, on the properties of plasma membrane mechanics of MDCK II cells. A: Membrane tension $T_{0}, T_{t}$ (B) and apparent area compressibility modulus (C) of MDCK II cells after cholesterol depletion from the plasma membrane.

Probing the mechanical properties of MBCD treated cells (Figure 47) reveals tension values of the apical membrane ( $T_{0}$ and $T_{t}$ ) that continuously increase while depleting cholesterol from the membrane, which finally leads to a reduction in excess apical membrane area. As a consequence, the overall apparent area compressibility module $\tilde{K}_{A}$ increases due to a decrease of $A_{e x}$, but maintains its bimodal distribution with a shifted weight to the larger $\tilde{K}_{A}$ value (Table 9).

Table 9: Mechanical parameters of confluent MDCK II cells obtained from force cycle analysis after exposure to MBCD. Values represent the maximum of a Gaussian curve fitted to the histograms.

\begin{tabular}{|c|c|c|c|c|}
\hline \multirow[b]{2}{*}{ peak max } & \multicolumn{2}{|c|}{$\tilde{K}_{A} / \mathrm{N} \mathrm{m}^{-1}$} & $T_{0} / \mathrm{mN} \mathrm{m}^{-1}$ & $T_{\mathrm{t}} / \mathrm{mN} \mathrm{m}^{-1}$ \\
\hline & I & II & I II & I II \\
\hline control & 0.02 & 0.09 & 0.23 & 0.27 \\
\hline $\operatorname{MBCD}(1 \mathrm{~h})$ & 0.02 & 0.12 & 0.52 & 0.80 \\
\hline $\operatorname{MBCD}(2 \mathrm{~h})$ & 0.05 & 0.19 & 0.64 & 0.68 \\
\hline MBCD (3h) & 0.13 & 0.54 & 0.52 & 0.76 \\
\hline
\end{tabular}




\subsubsection{Discussion}

If in-plane stretching of the membrane is indeed compensated by drawing additional membrane from a highly dynamic surface area reservoir [149], a stimulated loss of these reservoirs should lead to a larger overall tension and disappearance of low $\tilde{K}_{A}$ values. One conceivable experiment is the removal of cholesterol from the plasma membrane. Cholesterol depletion leads to dispersion of lipid rafts and therefore to removal of caveolae and microvilli acting as potential surface area reservoirs for the apical cell membrane [43, 144]. In detail, Cholesterol depletion from the cell surface causes the associated uncoupling of the cytoskeleton from the membrane. It is believed that ezrin establishes a strong connection between the plasma membrane and the cortical actin by binding to $\mathrm{PIP}_{2}$, which is also largely responsible for its activation, i.e. conformational switching from the dormant into the active state [44, 150-152]. Cholesterol depletion and therefore destruction of lipid raft domains delocalizes $\mathrm{PIP}_{2}$, resulting in a dissociation of cytoskeleton proteins from the membrane [45, 144, 153]. Immunostaining of ezrin and F-actin shows the reduction of these proteins during incubation with cholesterol depleting MBCD and AFMand SEM-imaging visualizes the disappearance of the membrane protrusions. This membrane reduction could be quantified by monitoring time resolved changes in membrane capacitance, which are proportional to membrane area at the applied frequency, together with an increased endocytosis rate.

The reduction in membrane area shifts the area compressibility module to higher values, almost by factor of five. The strongest and most important finding is that the lower values attributed to areas with excess membrane disappear, while the larger area compressibility most likely represents membrane regions without associated membrane reservoir (Table 9). The reduction of membrane reservoirs clearly results in a significant increase in membrane tension $\left(T_{0}\right.$ and $\left.T_{t}\right)$ by a factor of two.

This change in cellular elasticity is also confirmed by using the Hertzian approach to analyze the mechanical data. Here, cell stiffness expressed by Young's modulus increases by a factor of 4 . Similar results are found by Byfield and coworkers where they show a decrease in membrane deformability of aortic endothelium cells after cholesterol depletion [148]. 


\subsection{Disrupting the Cortical Integrity ${ }^{4}$}

Tension in the plasma membrane is only maintained by a fully functional actomyosin cortex. To investigate the impact of actomyosin integrity on plasma membrane tension, the cytoskeleton is disrupted by the addition of cytochalasin D. Further blebbistatin as an inhibitor of myosin II is used.

\subsubsection{Disassembly of F-actin with Cytochalasin}

Disrupting the actin cortex by exposing the cell monolayer to cytochalasin D (10 $\mu \mathrm{M})$ for $15 \mathrm{~min}$ promotes the depolymerization of the filaments. The change in the cortical structure is visible in the fluorescence micrographs shown in Figure 48. When treated with cytochalasin D, F-actin and ezrin distribution is altered, showing a more aggregated and clustered arrangement (Figure 48 C, D).
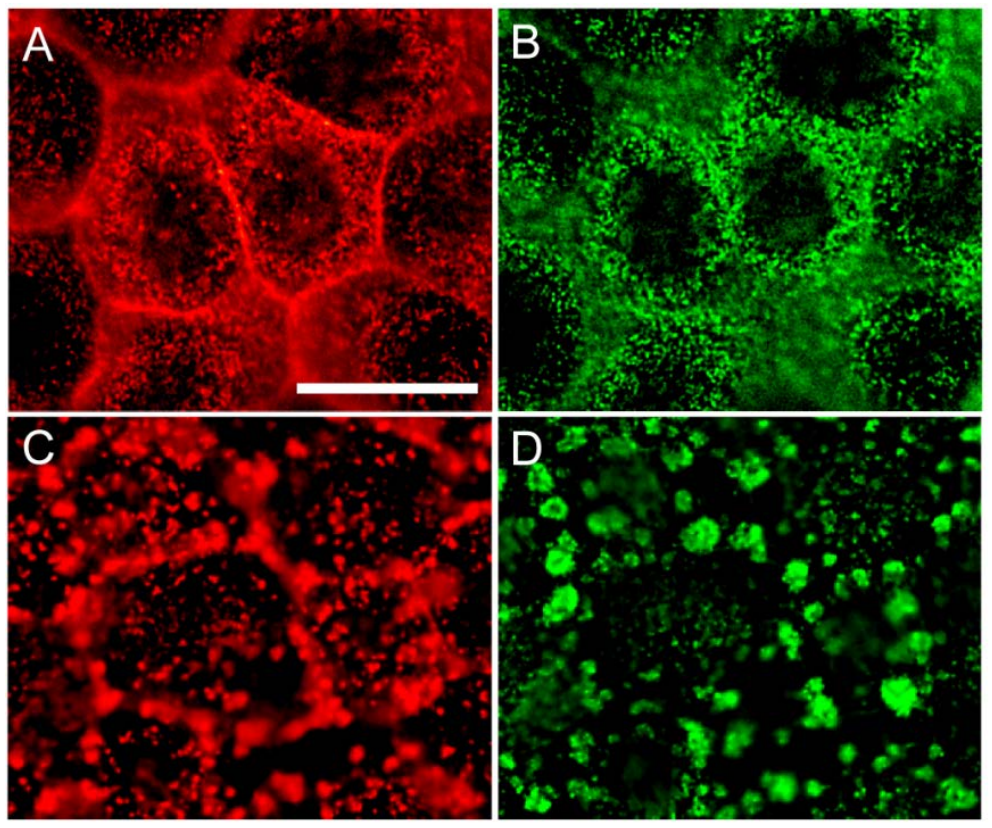

Figure 48: Fluorescence micrographs of MDCK II cells labeled for F-actin (red) and ezrin (green). Untreated cells $(A, B)$ and cytochalasin D treated cells (C, D). Scale bar $20 \mu \mathrm{m}$.

As a result of these structural changes, $\tilde{K}_{A}$ reflecting the existence of excess surface area, maintains its bimodal distribution centered at $0.02 \mathrm{~N} \mathrm{~m}^{-1}$ and $0.05 \mathrm{~N} \mathrm{~m}^{-1}$ with a shift of maximum II to slightly lower values compared to untreated cells which might be attributed

\footnotetext{
${ }^{4}$ Parts of this chapter are published in

Pietuch, A.; Brückner, B.R.; Fine, T.; Mey.I., Janshoff, A. (2013) Elastic properties of cells in the context of confluent cell monolayers: impact of tension and surface area regulation. Soft Matter, 2013, 9 (48), 11490 11502.
} 
to a release of membrane area in response to cytoskeleton removal (Figure 49 A and B). In this case, the cells are not capable of compensating the decrease of tension by a change of the surface area.
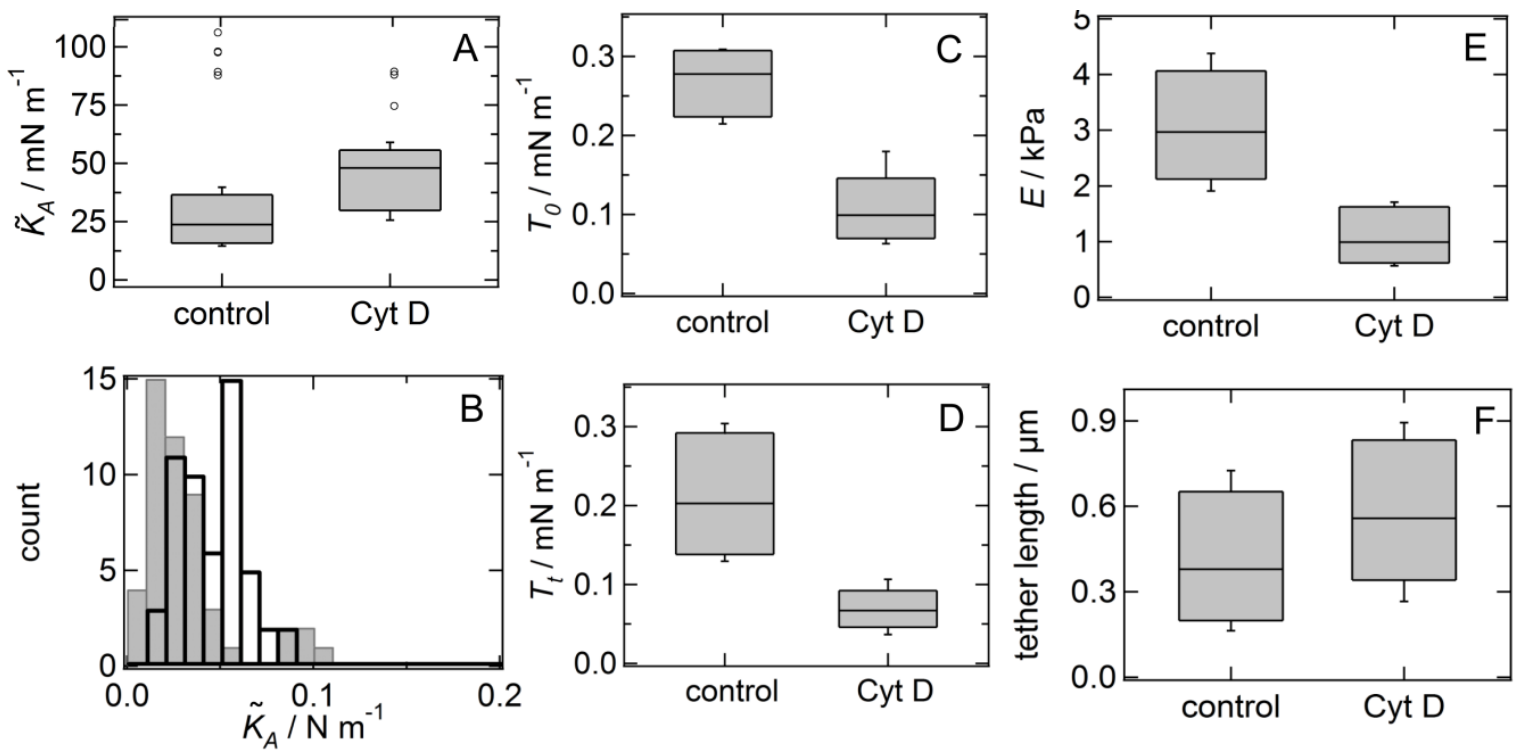

Figure 49: Mechanical investigation of MDCK II cells after disrupting the cellular cortex with cytochalasin D. A: Area compressibility modulus. B: Histogram of the area compressibility modulus, gray bars demonstrates untreated cells. C: Membrane tension $T_{0}$ and $T_{t}(\mathrm{D})$. E: Young's modulus. F: Tether length.

In contrast, membrane tensions $T_{0}$ and $T_{t}$ dropped substantially $\left(T_{0 \text {, control }}=0.27 \mathrm{mNm}^{-1} \pm\right.$ $0.06 \mathrm{mNm}^{-1} ; T_{0, \text { cyt }}=0.11 \mathrm{mNm}^{-1} \pm 0.06 ; T_{t, \text { control }}=0.22 \mathrm{mNm}^{-1} \pm 0.12 \mathrm{mNm}^{-1}, T_{t, \text { cyt }}=0.08$ $\mathrm{mNm}-1 \pm 0.04 \mathrm{mNm}-1$ ) confirming the crucial role of the actin network for establishing a finite lateral tension in the plasma membrane. Interestingly, the length of pulled tether rises when cells are subjected to F-actin disrupting agent, which also reflects a change in membrane cytoskeleton adhesion.

Concomitant to the decrease in measured membrane tension the Young's modulus decreases from $3.2 \pm 1.5 \mathrm{kPa}$ to a value of $1.3 \pm 1.0 \mathrm{kPa}$ (Figure $49 \mathrm{~F}$ ).

\subsubsection{Disrupting Actomyosin Connections with Blebbistatin}

Myosin generates tension within the cytoskeleton by crosslinking actin filaments and thus contracting the actin cytoskeleton. Blebbistatin directly inhibits the interplay between myosin and actin [154]. To examine to what extent the actomyosin connection affect the mechanical properties of the plasma membrane of MDCK II cells, blebbistatin is added in a final concentration of $50 \mu \mathrm{M}$ for $30 \mathrm{~min}$. 
Fluorescence microscopy images show no change in the ezrin and F-actin distribution on the cellular apex after exposure to blebbistatin (Figure 50 A, B). However, at the basal part of the cells, huge blebs are observable (Figure $50 \mathrm{C}$ ). Blebs are membrane outgrowths provoked by a local weakening of the cortical complex and promoted by the internal pressure of the cell, but normally described as lacking F-actin support [155]. F-actin is available at the stabilized state or during bleb retraction [156]. The generation of blebs is often due to a drop in tension.
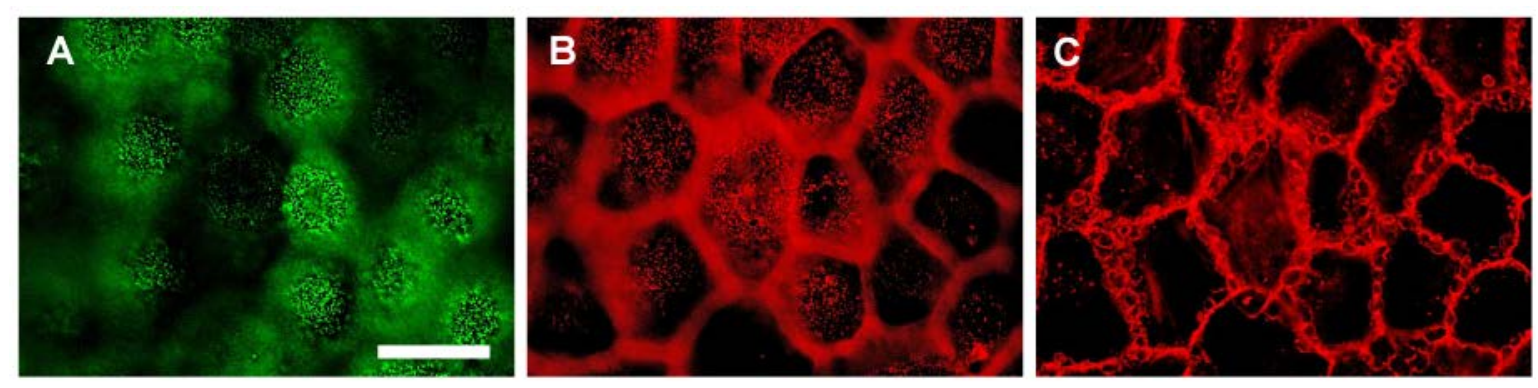

Figure 50: Fluorescence micrographs of MDCK II cells treated with blebbistatin. A: Ezrin is labeled in green showing protein distribution at the cellular apex. B: F-actin is labeled in red. C: F-actin in the basolateral region of the cells.

Changes in the mechanical properties of the plasma membrane are examined by indentation and tether pulling experiments after the addition of myosin inhibiting blebbistatin (Table 10).

Table 10: Mechanical parameter after cell treatment with blebbistatin. Mean values are represented with standard deviation.

\begin{tabular}{cccc}
\cline { 2 - 4 } & $\tilde{K}_{A} / \mathrm{N} \mathrm{m}^{-1}$ & $T_{0} / \mathrm{mN} \mathrm{m}^{-1}$ & $T_{t} / \mathrm{mN} \mathrm{m}^{-1}$ \\
\hline control & $\begin{array}{c}0.29 \pm 0.17 \\
(n=35)\end{array}$ & $\begin{array}{c}0.36 \pm 0.16 \\
(n=35)\end{array}$ & $0.39 \pm 0.2$ \\
blebb & $\begin{array}{c}0.23 \pm 0.16 \\
(n=35)\end{array}$ & $\begin{array}{c}0.29 \pm 0.13 \\
(n=50)\end{array}$ & $\begin{array}{c}0.43 \pm 0.21 \\
(n=97)\end{array}$ \\
\hline
\end{tabular}

A low but significant reduction of the lateral tension $T_{0}$ by a factor of 1.2 is found (Wilcoxon Rank test $\mathrm{p}=0.04$ ). Concomitantly, the area compressibility modulus decreases, which reflects an enlargement in available membrane area due to tension release.

In-plane tension obtained from tether pulling experiments do not change significantly during incubation with blebbistatin (Wilcoxon Rank test $\mathrm{p}=0.1$ ). This confirms the 
findings obtained from fluorescence microscopy, where no alteration in the apical ezrin and F-actin organization was found (Figure $50 \mathrm{~A}, \mathrm{~B}$ ).

\subsubsection{Discussion}

Disrupting the F-actin cytoskeleton with cytochalasin D leads beside a structural rearrangement of the apical F-actin to a change in the distribution of the co-localized protein ezrin. This alteration has great impact on the mechanical parameters, since the underlying cortex maintain the membrane tension. The depolymerization of F-actin decreases the membrane tension by a factor of 3. Additionally the Young's modulus is estimated showing also a reduction by a factor of 3. Rotsch and Radmacher reported the same decrease in the Young's modulus after cytochalasin D treatment of NRK cells [157]. The alteration in cortex integrity shows also changes of available membrane area on the plasma membrane, manifested in a slight lowering of peak II, while peak I representing lower $\tilde{K}_{A}$ values remains unaffected (Figure 49). Hence, we can attribute the lower $\tilde{K}_{A}$ values, of untreated MDCK II cells, to areas on the cell membrane with no or little adhesive support and therefore accessibility to excess membrane area. The increase in measured tether lengths supports the observation of a weakening in the membranecytoskeleton attachment [158].

Myosin II mainly interacts with F-actin bundled as stress fibers in the basal part of the cell [159] [160]. By varying this force generating arrangement, no change in the architecture of the cellular apex (ezrin and F-actin distribution) and measured in-plane tension from tether pulling experiments are found. However, tremendous structural alteration is observed in the basal part, where huge blebs are appearing during blebbistatin treatment. Blebs are known to nucleate at regions where the membrane cytoskeleton adhesion is weakened ([155] [10]). Although basal changes are not detected in these experiments by tether pulling, changes in membrane tension determined by indentation experiments display a decrease [161]. Simultaneously, the area compressibility modulus is declined, which can be attributed to an increase in available membrane area due to the lowered membrane tension. 


\subsection{Single Cell Manipulation}

When cells are subjected to chemical stimuli by adding a substance directly to the culture medium the entire cell layer reacts. This prohibits studying mechanical responses of individual cells in an intact, i.e. viable surrounding. Therefore, single cell experiments are devised to address the question to what extent the membrane-cortex-adhesion contributes to the mechanical behavior of one cell within a non-affected cell monolayer, mimicking the change of one cell upon genetic dysfunction. The target protein is ezrin, a member of the ERM family, which is responsible for connecting F-actin to the plasma membrane by specifically binding to $\mathrm{PIP}_{2}$.

\subsubsection{Uncoupling the Membrane from the Cytoskeleton}

The cationic antibiotic neomycin is injected into a single MDCK II cell of a confluent MDCK II monolayer. Neomycin specifically forms complexes with $\mathrm{PIP}_{2}$ [116] and therefore efficiently disconnects the membrane from the actin cortex (Figure 51).

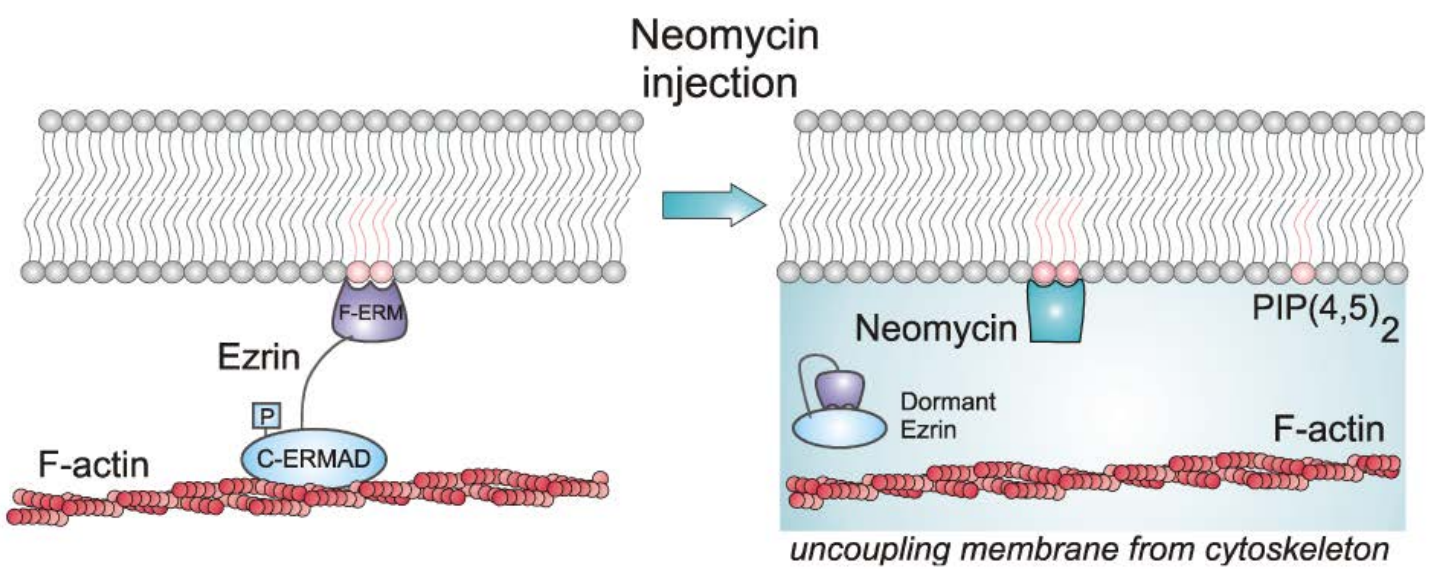

Figure 51: Schematic illustration of the uncoupling of the plasma membrane from the cytoskeleton by neomycin injection due to binding of neomycin to $\mathrm{PIP}_{2}$.

\subsubsection{Morphological Alteration after Neomycin Injection}

After the microinjection of neomycin into the cytoplasm of a single cell, the inherent roughness of the apical cell surface due to microvilli and other protrusions diminished solely on the injected cell (Figure $52 \mathrm{~A}-\mathrm{C}$ ). 

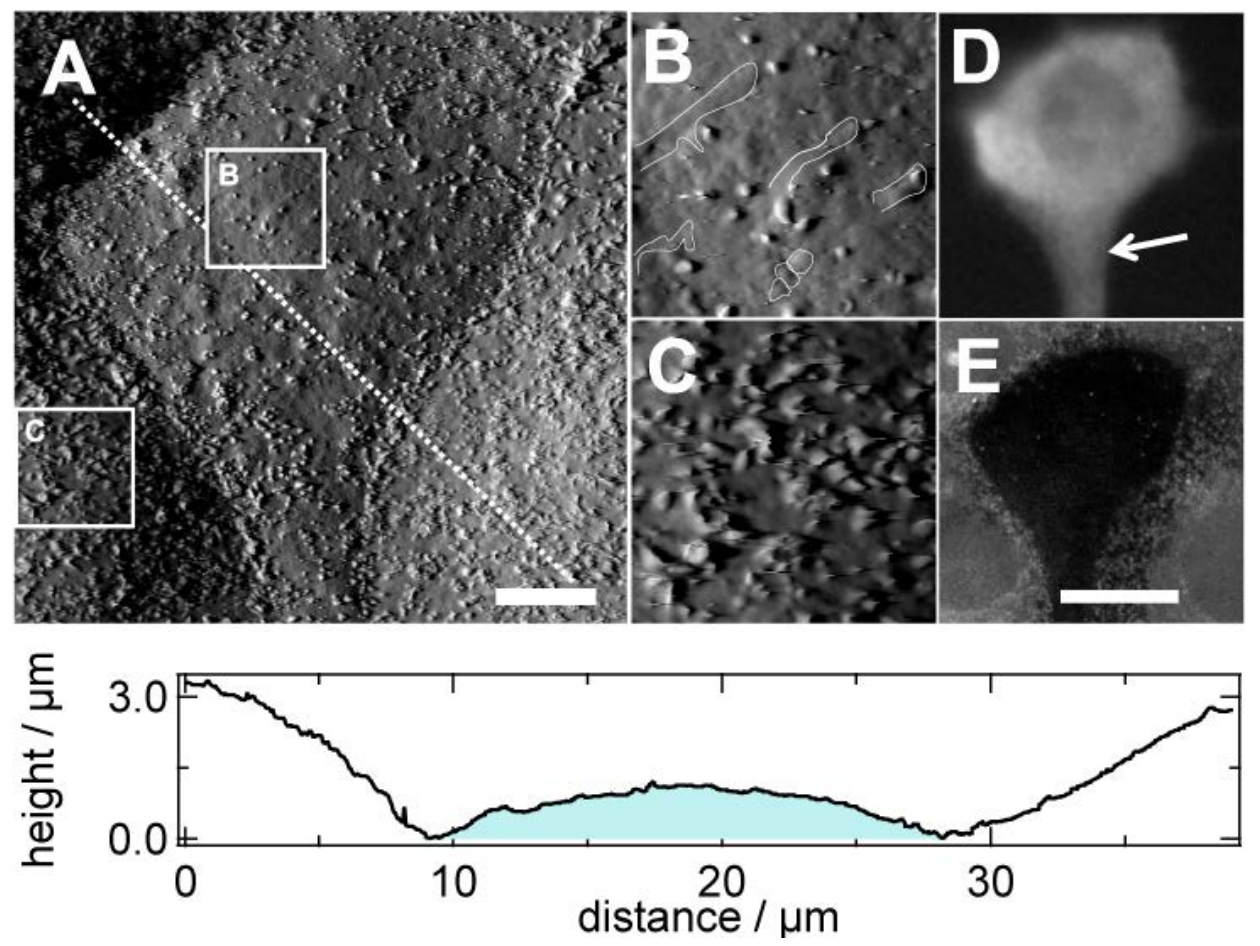

Figure 52: Microinjection of neomycin into a single MDCK II cell of a confluent monolayer. A: AFM deflection image showing substantial alteration in plasma membrane topography $4 \mathrm{~h}$ after neomycin injection (loss of microvilli). The line profile taken along the dotted line shows that the cell height of the injected cell is reduced compared to its adjacent neighbors. Scale bar: $5 \mu \mathrm{m}$. B: Zoom-in of A showing details of the surface features of the injected cell. The cellular cortex bulges beneath the plasma membrane as highlighted by white markers. C: Surface details of adjacent untreated cell (control). E: The injected cell is negative for immunostaining of ezrin. D: Fluorescence image of co-injected FITC-dextran for the localization of the injected cell.

As expected, the injected cell is negative for the immunostaining of ezrin, indicating a loss of cross-linking ezrin at the plasma membrane (Figure $52 \mathrm{E}$ ). Besides the reduction of microvilli, the cell is significantly reduced in height compared to their adjacent neighbors (Figure $52 \mathrm{~A}$, linescan). The axial shrinkage is assigned to a reduced distance of the apical membrane from the actin cortex similar to what is found if the cells are exposed to hyperosmolar stress forcing the membrane to collapse onto the cytoskeleton [130]. This effect is observable in section 5.6 when cells are subjected to a hyperosmolar solution (Figure 68). As a consequence, topographical features of the terminal web becomes visible on the surface, which are attributed to the cellular cortex being forced into close contact with the cell membrane and therefore bulging below it (Figure $52 \mathrm{~B}$ ). These new features are structurally comparable with those found when the membrane of MDCK II cells is dissolved with detergents, exposing the cortical organization under the plasma membrane (Figure 53). 


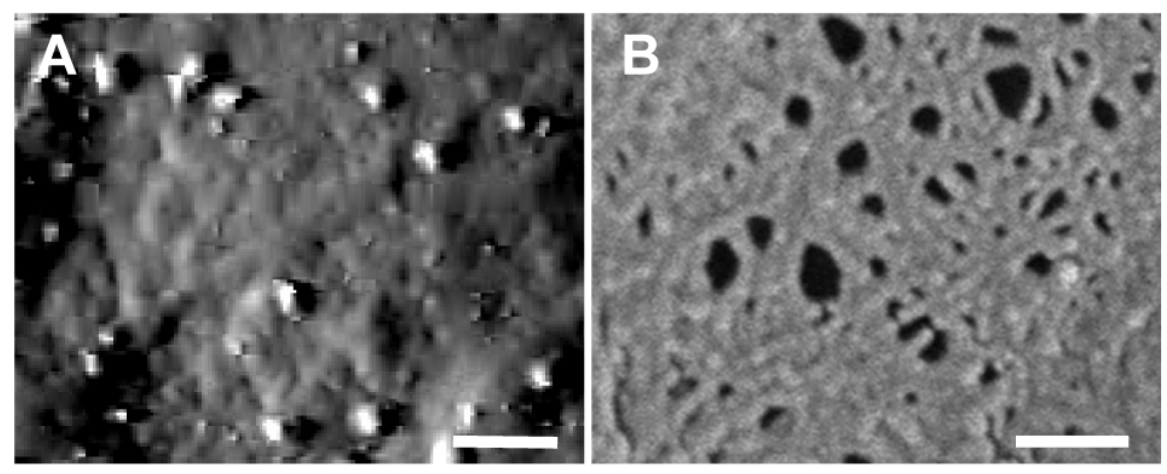

Figure 53: Topological features of the plasma membrane of MDCK II cells. A: Deflection image of neomycin injected cell. B: SEM image of TriX treated MDCK II cells, exposing the terminal web under the dissolved plasma membrane. Scale bar in A is $1 \mu \mathrm{m}$ in B $0.1 \mu \mathrm{m}$.

Beside the topological changes of the plasma membrane it should be noted, that a common morphological characteristic of cells injected with neomycin is the development of a tail like protrusion (Figure 52 D, arrow).

\subsubsection{Mechanical Properties of Neomycin Injected Cells}

Microinjection of neomycin greatly influences not only the morphology of the whole cell such as the topography of the plasma membrane, but also its mechanical properties. The experimental setup allows to directly compare the cellular mechanics of an untreated cell adjacent to the injected one. The force map is converted in a Young's modulus map by applying the Hertz-model to the force distance curves (Figure 54).
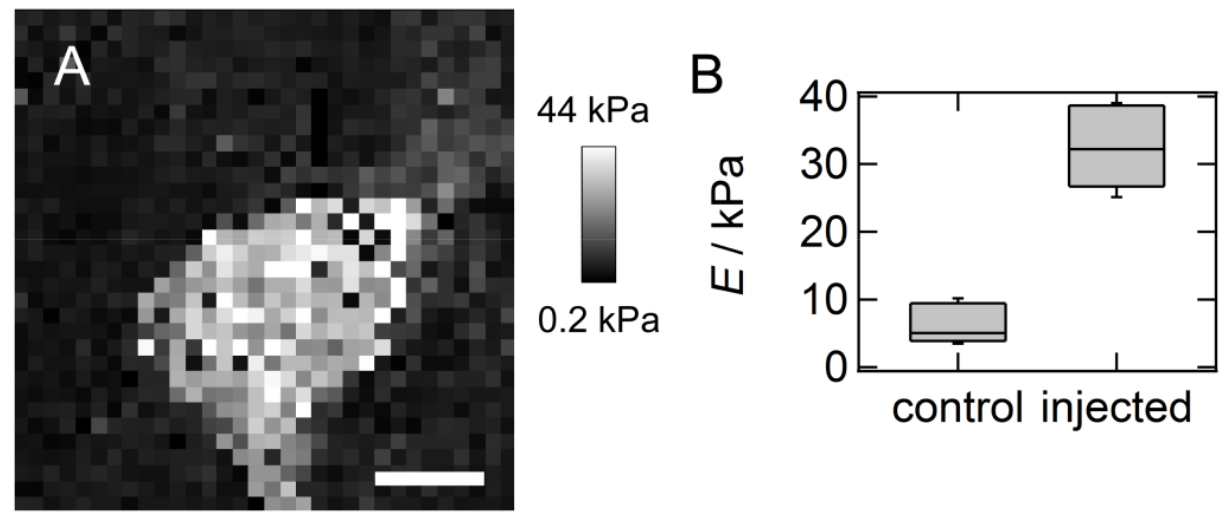

Figure 54: Analysis of neomycin injected cell (4h after injection) with the Hertzian approach: A: Young's modulus map. Scale bar $10 \mu \mathrm{m}$. B: $E$-values of adjacent untreated cells and injected one.

The stiffness of the injected cells is greatly increased up to a value of $32 \mathrm{kPa} \pm 12 \mathrm{kPa}$ (4h after injection). The Young's modulus of the adjacent untreated cells is $6.4 \mathrm{kPa} \pm 3.5 \mathrm{kPa}$. This is an increase by a factor of 5 . 
The mechanical changes in cell membrane properties after injection are also analyzed by using the extended liquid droplet model for adherent and confluent cells, yielding the lateral tensions $T_{0}$ and the area compressibility modulus $\tilde{K}_{A}$ from indentation experiments and from tether pulling experiments $\left(T_{t}\right)$ (Figure 55), respectively.

A
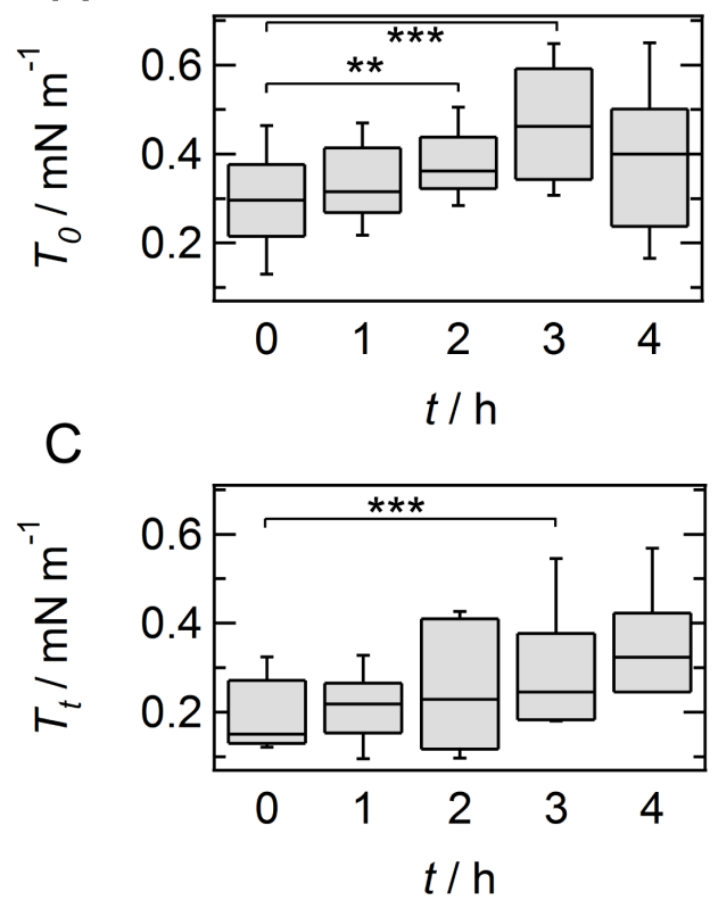
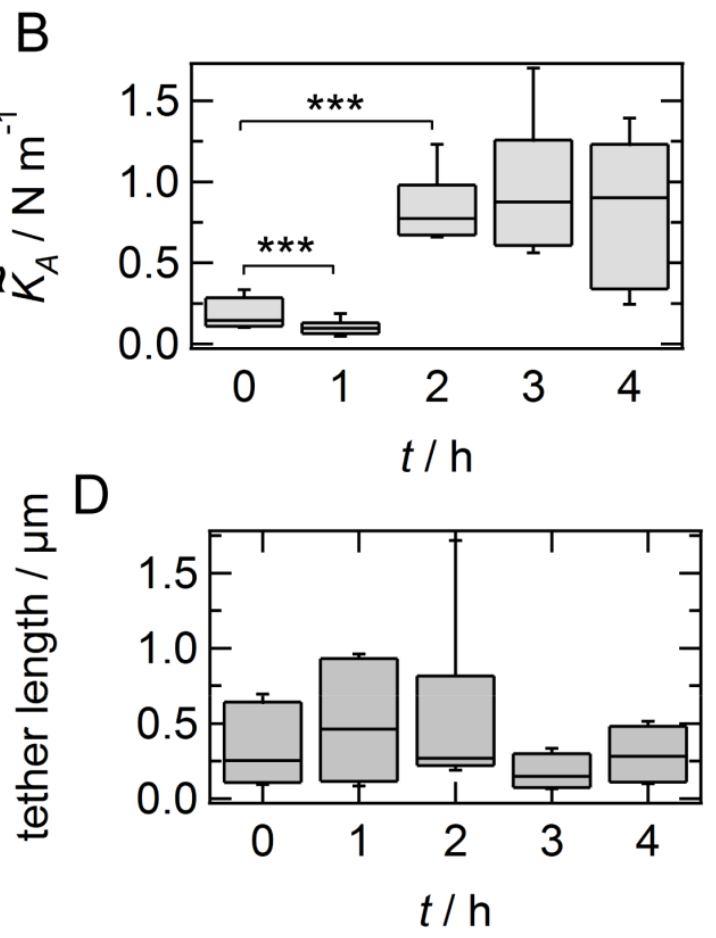

Figure 55: Mechanical parameters ( $\tilde{K}_{A}, T_{0}$ and $T_{\mathrm{t}}$ ) after injection of neomycin. (** correspond to $\mathrm{p}<10^{-2} ; * * *$ to $\mathrm{p}<10^{-3}$, two-tailed Wilcoxon rank sum test). D: Boxplot of tether lengths from tether pulling experiments before and after the injection of neomycin.

Both tension values increase over time after injection of neomycin $\left(T_{0}{ }^{0 h}=0.29 \mathrm{mN} \mathrm{m}^{-1}\right.$, $T_{0}^{4 h}=0.4 \mathrm{mN} \mathrm{m}^{-1} ; T_{t}^{0 h}=0.15 \mathrm{mN} \mathrm{m}^{-1}, T_{t}^{4 h}=0.32 \mathrm{mN} \mathrm{m}^{-1}$, median). The apparent area compressibility module immediately after injection $\left(\tilde{K}_{A}^{\text {th }}\right)$ is lower compared to untreated cells and lacks bimodal distribution indicative of a sudden release of excess membrane. This result is in agreement with tether length distributions (tether length at $1 \mathrm{~h}>$ tether length at $0 \mathrm{~h}, 2 \mathrm{~h}, 3 \mathrm{~h}, 4 \mathrm{~h}$; Figure $55 \mathrm{D}$ ). The tether length serves as an indicator of excess surface area, i.e. large tether lengths correspond to larger membrane reservoirs.

The apparent area compressibility $\tilde{K}_{A}$ measured more than $2 \mathrm{~h}$ after neomycin injection is large enough to safely exclude the availability of any excess surface area buried in 
invaginations or protrusions of the apical membrane. This is similar to the experiments using MBCD but even more pronounced. The increase in $\tilde{K}_{A}$ is interpret as a decrease in $A_{e x}$ since the cell responds to a sudden drop in tension after neomycin injection by removing excess area. The restored tension occurs at the expense of surface area loss.

\subsubsection{Cell Stiffening Depends on Ezrin Disappearance}

During the experiments concerning the microinjection of neomycin, one of the injected cells displays an inhomogeneous distribution of the protein ezrin (Figure 56). Distinct areas of the cellular surface of the injected cell appear almost smooth in the topological image (Figure 56 A, Region 3). In this peripheral region, ezrin is almost completely vanished. However, in the cells' middle (region 2) the linker protein is still detectable (Figure 56 B).
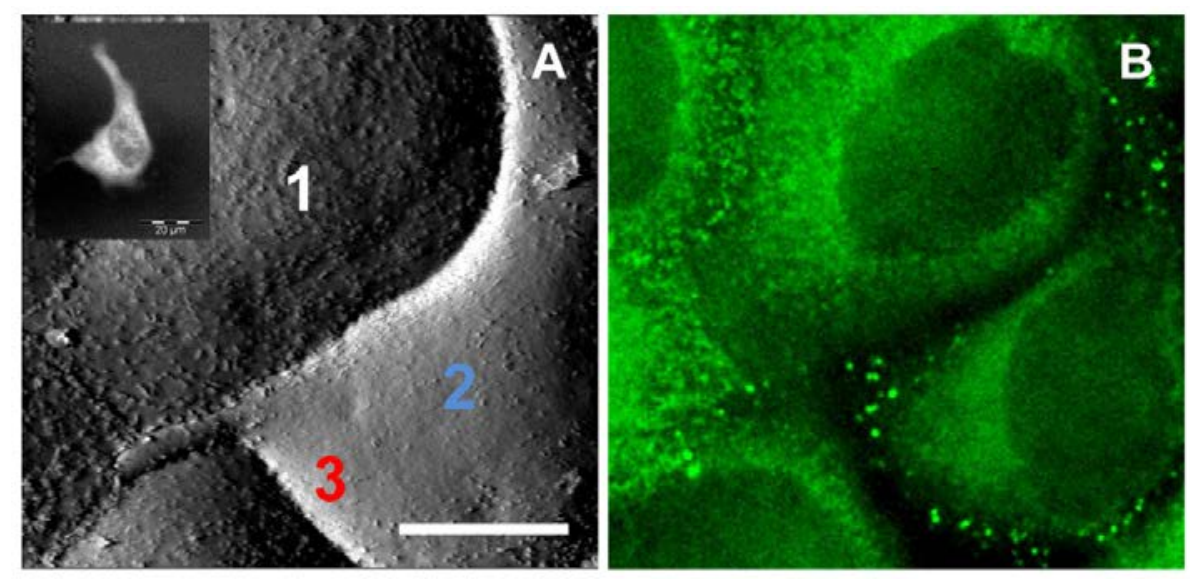

Figure 56: A: AFM deflection image of neomycin injected MDCK II cell 4h after injection. Scale bar $10 \mu \mathrm{m}$. Inlay shows the fluorescence micrograph of the co-injected FITC-dextran. B: Fluorescence micrograph of the immunostaining of ezrin of a neomycin injected MDCK II cell.

This irregular distribution of the protein enables to investigate the contribution from the membrane-cytoskeleton linker ezrin to the mechanical properties of the plasma membrane. Therefore, AFM indentation experiments are performed on this partly altered cell (Figure 57). The sample is divided into three regions. Region 1 represents unaffected adjacent cells. Region 2 marks the middle of the injected cell, where ezrin concentration is lower but still detectable and region 3 with just isolated green fluorescence dots indicating ezrin presence. 
Mechanical parameters obtained from fitting the extended liquid droplet model to the experimental data show mechanical differences in the three areas.
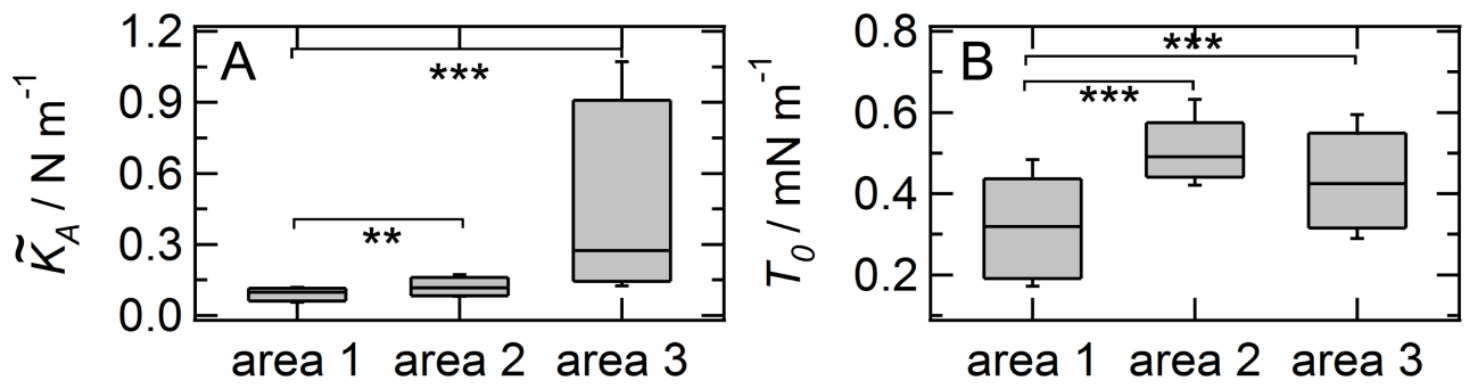

Figure 57: Mechanical parameter obtained from indentation experiments of a neomycin injected MDCK II cell. Area 1 corresponds to untreated adjacent cells. Area 2 belongs to regions on the injected cell where still ezrin protein is detectable. Region 3 is mainly devoid of ezrin. (** Correspond to $\mathrm{p}<10^{-2}$; ${ }_{* * *}$ to $\mathrm{p}<10^{-3}$, twotailed Wilcoxon rank sum test).

Region 2 which corresponds to the area on the plasma membrane with reduced ezrin density display elevated mechanical parameters indicating cell stiffening. The apparent area compressibility modulus $\tilde{K}_{A}$ increases from $0.09 \mathrm{~N} \mathrm{~m}^{-1} \pm 0.04 \mathrm{~N} \mathrm{~m}^{-1}$ to $0.14 \mathrm{~N} \mathrm{~m}^{-1} \pm$ $0.1 \mathrm{~N} \mathrm{~m}^{-1}$ (Figure $57 \mathrm{~A}$ ). The membrane tension rises simultaneously from $0.3 \mathrm{mN} \mathrm{m}^{-1} \pm$ $0.1 \mathrm{~N} \mathrm{~m}^{-1}$ to $0.5 \mathrm{mN} \mathrm{m}^{-1} \pm 0.1 \mathrm{mN} \mathrm{m}^{-1}$ (Figure $57 \mathrm{~B}$ ). Regarding region 3, where almost no ezrin is visible in the fluorescence micrograph the mechanical investigation display an even higher value for the apparent area compressibility modulus $\left(\tilde{K}_{A}=0.5 \mathrm{~N} \mathrm{~m}^{-1} \pm 0.4 \mathrm{~N}\right.$ $\left.\mathrm{m}^{-1}\right)$, while membrane tension stays almost constant $\left(T_{0}=0.43 \mathrm{mN} \mathrm{m}^{-1} \pm 0.2 \mathrm{~N} \mathrm{~m}^{-1}\right)$.

This experiment shows the close connection between the presence of the protein ezrin at the cellular surface and the mechanical properties of the plasma membrane. Lower levels of ezrin can be attributed to smoother region of the cellular surface concomitantly with an increase in membrane tension and apparent area compressibility modulus. 


\subsubsection{Further Reactions of Neomycin Injected Cells}

In some experiments a different cellular reaction is observable as mentioned in the sections before, when cells are injected with $\mathrm{PIP}_{2}$ inhibiting neomycin. Injected cells which are not developing the above mentioned tail-like protrusion, but stay closed packed with adjacent cells, tend to became apoptotic (Figure 59). In the following examples cellular bodies reveal membrane outgrowths, also called blebs, which are indicative for apoptosis. In addition the nucleus is destroyed, as it is known for the programmed cell death (Figure 58, Figure 59) ([162]).
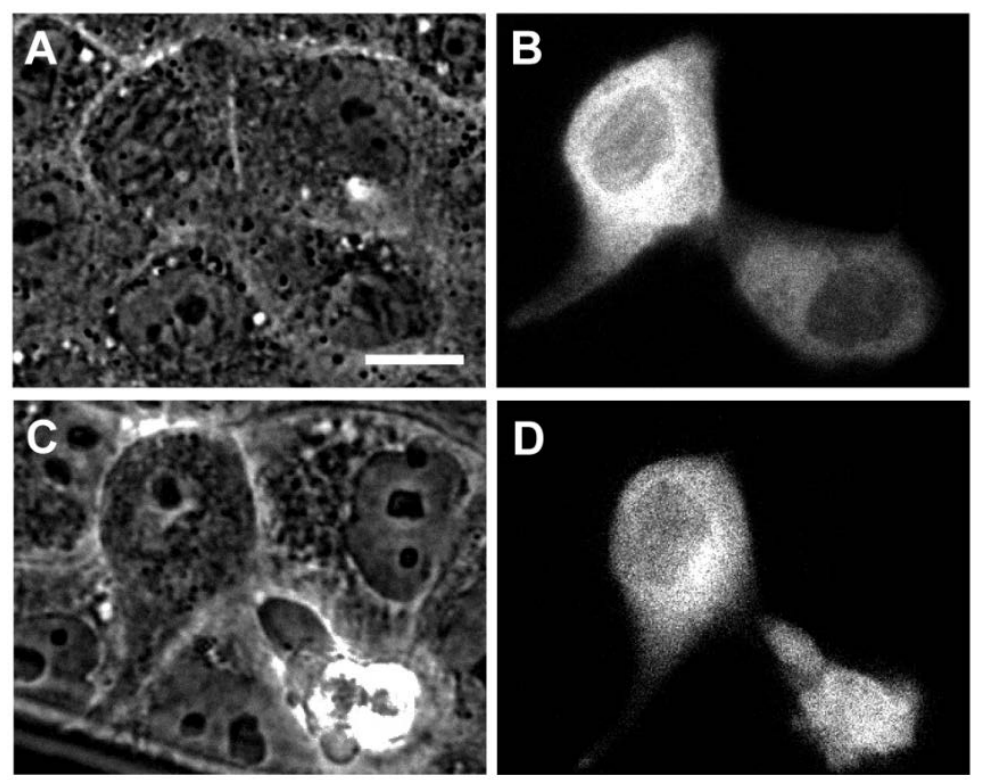

Figure 58: Cellular reaction of injection of neomycin. Cells frequently undergo apoptosis. A: Phase contrast micrograph of neomycin injected MDCK II cells (1h). B: Fluorescence micrograph of neomycin injected cells. The cells are co-injected with FITC-dextran. C: Phase contrast micrograph of neomycin injected cell after 6h. Cell in the lower right corner is apoptotic. D: Fluorescence micrograph of cell in C. Scale bar 10 $\mu \mathrm{m}$.

The membrane of the injected cell (right corner) display many blebs 6h after injection of neomycin (Figure 58), which is indicative for a programmed cells death. In comparison, the injected cell in the left corner illustrates again the frequently observed morphological alteration after the injection of neomycin. The cell develops a tail-like protrusion. 

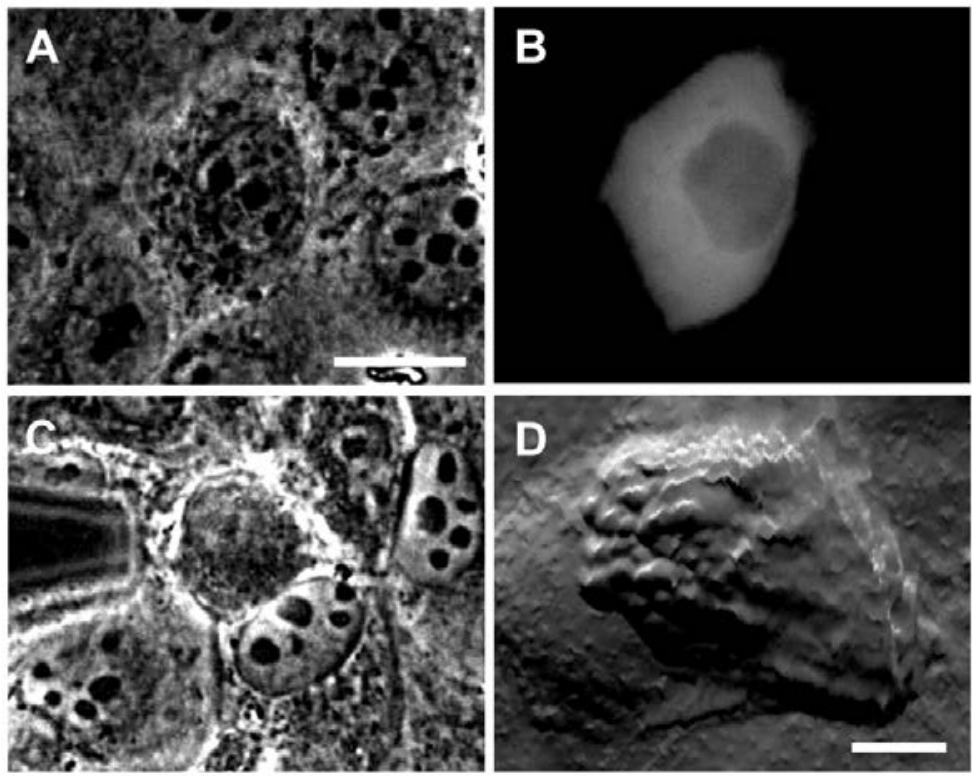

Figure 59: Cellular reaction of injection of neomycin. A: Phase contrast micrograph of neomycin injected MDCK II cells (1h). B: Fluorescence micrograph of neomycin injected cells. C: Phase contrast micrograph $8 \mathrm{~h}$ after injection. D: AFM deflection image of injected cell (8h). Scale bar $10 \mu \mathrm{m}$.

The mechanically investigation using conA coated tip for pulling out membrane nanotubes reveals a further feature of neomycin injected MDCK II cells: As indicated with white arrows in Figure 60, the manipulated cell shows membrane strings lying on the adjacent cells. This observation is explainable, since the injection of neomycin uncouples the plasma membrane from the cytoskeleton and enables to pull out longer membrane nanotubes. This particularity is never observed in experiments with untreated cells, supporting the finding, that the plasma membrane of MDCK II cells is efficiently uncoupled from the underlying cytoskeleton by microinjecting neomycin.
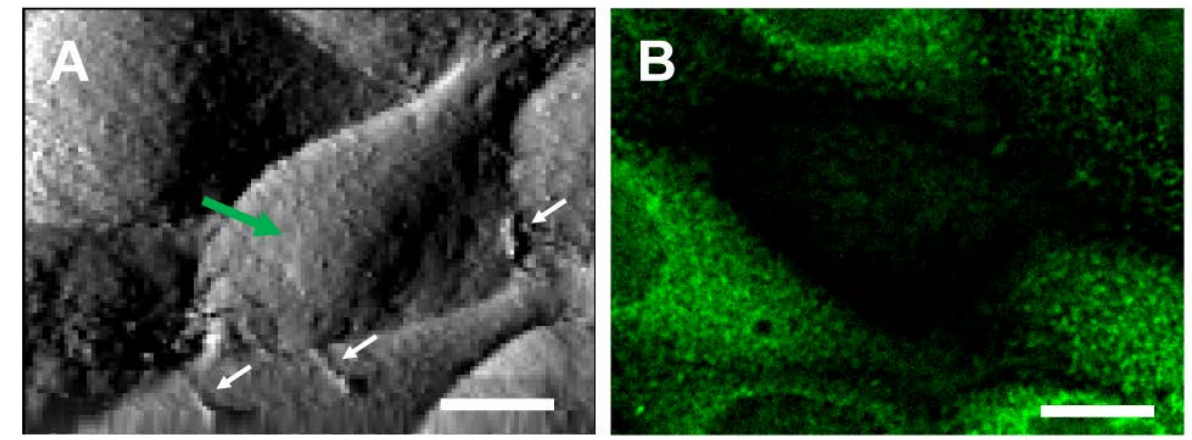

Figure 60: A: AFM deflection image of MDCK II cells after neomycin injection. Green arrow mark injected cell, white arrows show membrane strains pulled out of the plasma membrane of injected cells by a ConA coated cantilever tip. Scale bar $10 \mu \mathrm{m}$. B: Fluorescence micrograph of neomycin injected cell. Cells are labeled for ezrin. Injected cells display decreased level of ezrin. Scale bar $10 \mu \mathrm{m}$. 


\subsubsection{Discussion}

The specific uncoupling of membrane from the cortex by neomycin-injection into the cytosol of a single cell emphasizes the importance of membrane-cytoskeleton adhesion on the morphological characteristics of the epithelial cells and the mechanics of the plasma membrane. $\mathrm{PIP}_{2}$ inactivation leads to considerable changes in the morphology of the apical membrane such as the disappearance of microvilli accompanied by a loss in cell height as compared to untreated adjacent cells. Besides, injected cells frequently are developing taillike protrusions and occasionally undergo apoptosis.

The remodeling of the apical membrane reveals new topographical features on the cell surface probably due to collapsing of membrane onto the underlying actomyosin cortex. Since the terminal web does not significantly contribute to the area compressibility modulus of the plasma membrane due to its large extensibility $\left(K_{A}<0.001 \mathrm{~N} \mathrm{~m}^{-1}\right)$ we are measuring limiting values of $\tilde{K}_{A}$ for a plasma membrane in the absence of any surface area reservoirs [17]. Interestingly, the initial response of the cells directly after injection of neomycin provides evidence that prior to the collapse and straightening of the plasma membrane joining into a composite shell, a decrease in the apparent area compressibility modulus accompanied by an increase in pulled tether lengths occurs, indicative of a sudden availability of excess membrane area after removal of membrane cortex contacts. After this initial release of tension the cell compensates the loss of cross-linkers by removing excess area from the apical membrane producing larger $\tilde{K}_{A}$ values.

Due to the loss of membrane-cytoskeleton anchorage, because of inactivating $\mathrm{PIP}_{2}$, the manipulated cells starts to decrease the excess membrane area [17]. The reduction in membrane area, as intended by the increase in $\tilde{K}_{A}$ could not been quantified with the ECIS method was shown for cholesterol depletion with MBCD. Although the insertion of membrane impermeable neomycin was achieved by electroporation (data not shown), many cells detach from the gold electrode, maybe due to apoptosis as mentioned in section 5.4.1.4. This leads to a drastic change in the impedance signal and preclude the detection of changes in the cellular parameter $C_{m}$. From the morphological and mechanical similarities between MBCD treated cells and neomycin injected ones, it is inferred that the manipulation leads also to a membrane uptake and with this a change in membrane area. 
Important observations are the high rate of apoptotic cells, and the inability of manipulated cells to counteract the change in membrane area and mechanics. Generally, cells have the facility to regulate their membrane area (surface area regulation SAR), like membrane uptake when membrane tension gets low or exocytosis of internal membrane reserves when tension gets high [17]. A counteracting reaction concerning the membrane tension increase after neomycin injection is not observed. This is obviously due to the inhibition of $\mathrm{PIP}_{2}$, which is a important key factor for diverse cellular functions and processes.

The manipulation of one single MDCK II cell inside an intact monolayer leads to mechanical change of the injected cell, but no alteration in the adjacent ones is observed. 


\subsubsection{Increasing Number of Binding Sites for Ezrin ${ }^{5}$}

The microinjection of neomycin showed how cells response to the uncoupling of the plasma membrane from the underlying cytoskeleton. The inactivation of $\mathrm{PIP}_{2}$ leads to distinct changes of topography, morphology and mechanical behavior of MDCK II cells and emphasizes the importance of the membrane-cortex adhesion and its influence on the mechanical properties of the plasma membrane. The opposite approach is intended by injecting $\mathrm{PIP}_{2}$ directly into the cytosol of the cells and thereby increasing the number of binding sites between membrane and actin-cytoskeleton mediated by the anchor molecule ezrin. $\mathrm{PIP}_{2}$ comprises $~ 1 \%$ of the phospholipids in the plasma membrane [47]; whereas inactivated ezrin is present in the dormant state in the cytosol [48]. Therefore, it is reasonable to increase the amount of $\mathrm{PIP}_{2}$ rather than the amount of ezrin.

\subsubsection{Morphological Changes after Injection of $\mathrm{PIP}_{2}$}

The microinjection of $\mathrm{PIP}_{2}$-micelles into the cytoplasma of confluent MDCK II cells results in changes of the topography of the plasma membrane (Figure 61). The injected cells show a reduced presence of microvillar structure (Figure 61 C, D). Although these small protrusions are diminished larger features on the cell surface become visible on the apical plasma membrane.
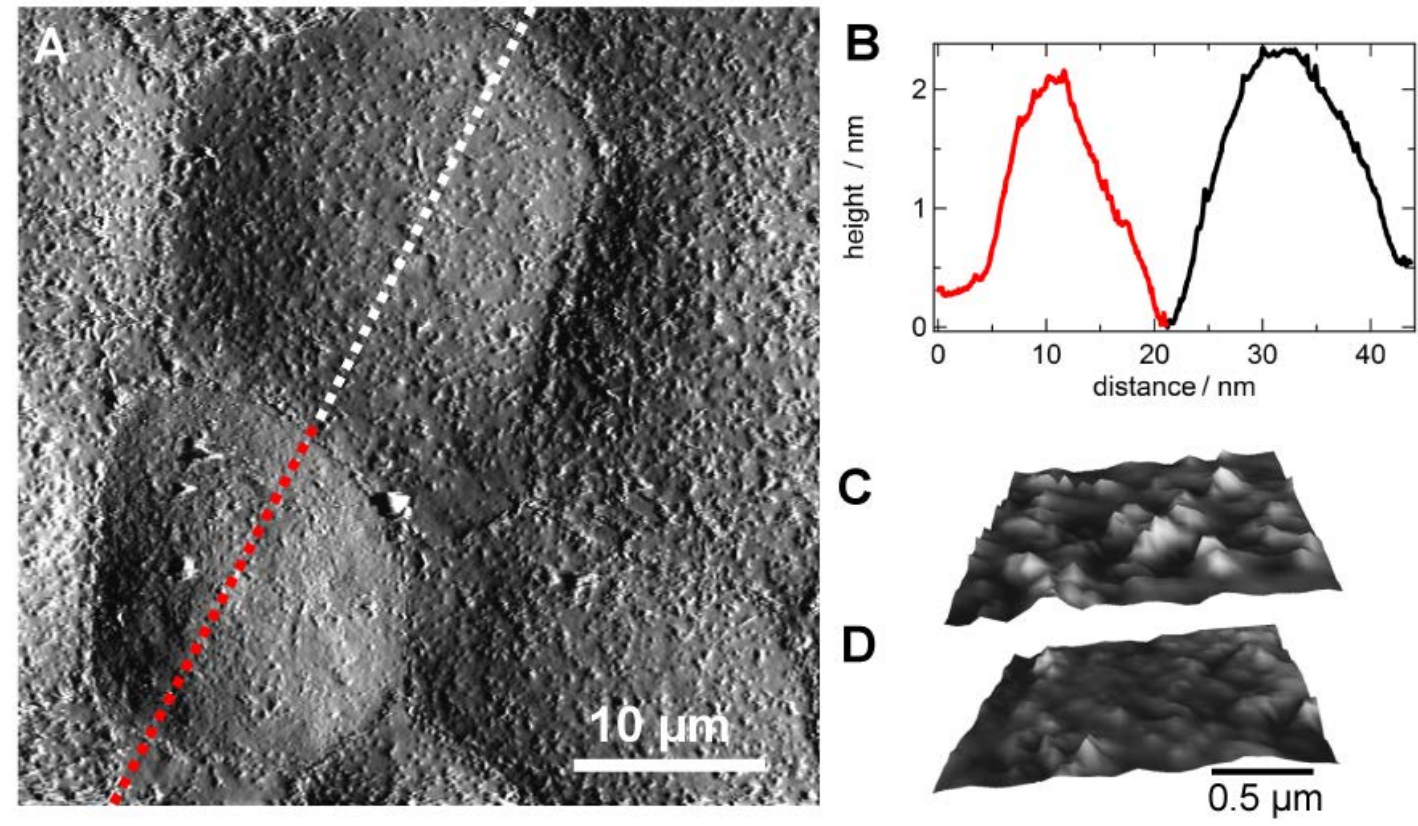

\footnotetext{
${ }^{5}$ Experiments and data processing was carried out by Bastian R. Brückner during his Master Thesis.
} 
Figure 61: Topographical alteration of confluent MDCK II cells after injection of $\mathrm{PIP}_{2}$ - micelles into a single cell. A: AFM deflection image after $\mathrm{PIP}_{2}$ injection. Red dashed line display injected cell, white untreated one. B: Line scan of A. C Three dimensional reconstruction of the AFM height image in A from untreated cell. Membrane protrusions are visible. D: 3-D reconstruction from height image in A of injected cell.

These features appear more aggregated and show a different organization than usually observed for microvilli. To find out whether a molecular alteration in the cortical structure causes the topographical changes, MDCK II cells are fluorescently labeled after the microinjection of $\mathrm{PIP}_{2}$ (Figure 62). The fluorescence micrographs confirm an enhanced presence of $\mathrm{PIP}_{2}$ in injected cells. Concomitantly, more ezrin and F-actin is found in the apical part of the manipulated cells. Ezrin accumulates in regions of increased $\mathrm{PIP}_{2}$ level. Along with an elevated level of $\mathrm{PIP}_{2}$ and ezrin, the organization of the F-actin is altered. Injected cells display an enhanced amount of F-actin, especially in the apical region of the cells (Figure $62 \mathrm{C}$ ).
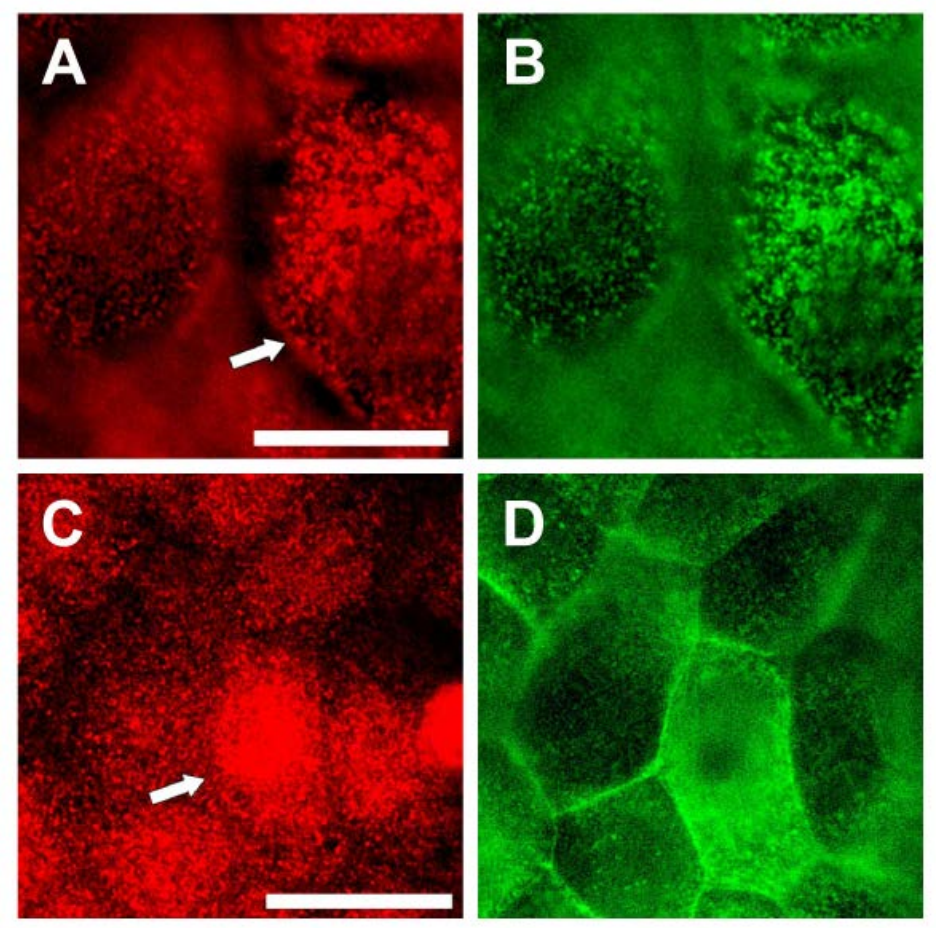

Figure 62: Fluorescence micrographs of MDCK II cells after PIP ${ }_{2}$ injection. A and C: Immunostaining of $\mathrm{PIP}_{2}$ after microinjection. Arrow marks the injected cell. Scale bar is $20 \mu \mathrm{m}$. B: Ezrin of injected and untreated cells are immunostained with Alexa Fluor 488. D: F-actin cytoskeleton is stained with Alexa Fluor 488.

It should be pointed out, that the remodeling of the cellular apex does not lead to a shape change of the cells, as found for neomcyin injected cells. Injected cells show no alteration in cell height or radius (Figure $61 \mathrm{~B}$ ). 


\subsubsection{Mechanical Investigation of PIP $_{2}$ Injected Cells}

To examine whether the injection of $\mathrm{PIP}_{2}$ and the accompanied increase in ezrin and Factin at the cellular apex has a influence on the mechanical properties of the plasma membrane of MDCK II cells, site-specific indentation experiments are carried out. Mechanical alteration is analyzed by fitting the extended liquid droplet model for adhering and confluent cells to the experimental data, providing the membrane tension $T_{0}$ and the apparent area compressibility modulus $\tilde{K}_{A}$.

After injecting $\mathrm{PIP}_{2}$-micelles into the cytoplasm of the cells, force curves are recorded consciously over $5 \mathrm{~h}$. This chemical manipulation of the plasma has a substantial impact on the mechanical properties of the plasma membrane (Figure 63).
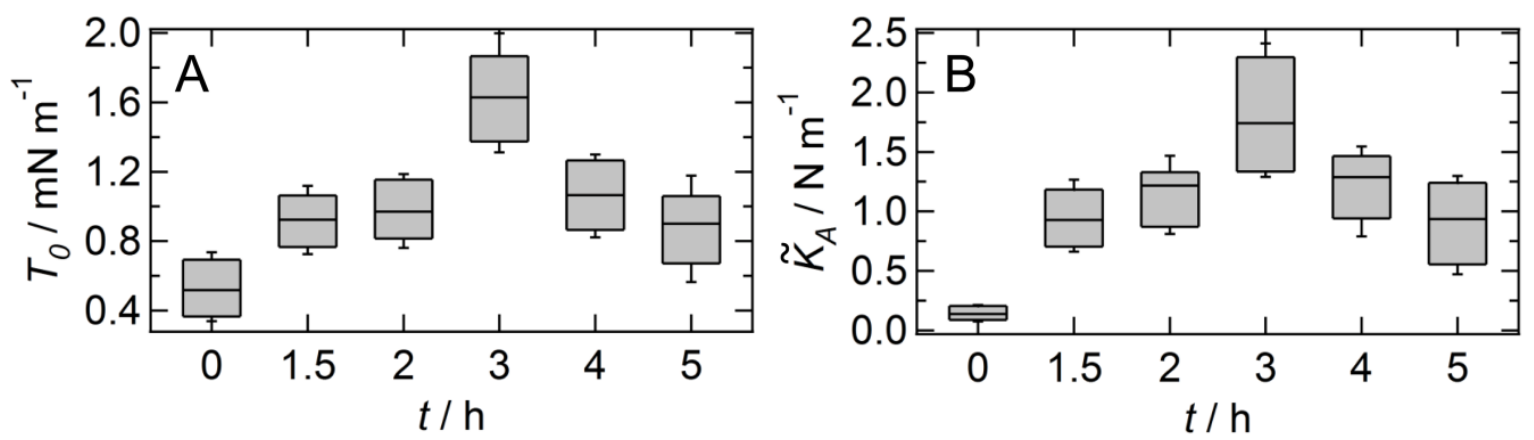

Figure 63: Mechanical investigation of MDCK II cells after injecting PIP $_{2}$-micelles, at different time points after injection. A: Membrane pre-tension $T_{0}$. B: Apparent area compressibility modulus.

The manipulation of the plasma membrane, precisely, the increase of ezrin binding sites along with the reorganization of the cortical structure, leads to an increase in tension and apparent area compressibility modulus of the plasma membrane. The pre-tension $T_{0}$ already rises by factor of almost 2 after $1.5 \mathrm{~h}$. The maximal $T_{0}$-value is found $3 \mathrm{~h}$ after microinjection. Apparently, this time is needed to reorganize the cortex. After 3h pretension decreases again, but does not reach the previously value measured for untreated cells. Simultaneously, the apparent area compressibility modulus increases, reflecting a decrease in available excess membrane area. Likewise the membrane tension, $\tilde{K}_{A}$ declines after $3 \mathrm{~h}$. The most prominent difference between microinjection of neomycin and $\mathrm{PIP}_{2}$ is the decline of the mechanical parameters $3 \mathrm{~h}$ after injection. The manipulated cells seem to counteract the reinforced mechanical situation, i.e. by reformation of membrane reservoir the original tension is restored. Such a reaction is never observed after neomycin injection. 


\subsubsection{Discussion}

By injecting $\mathrm{PIP}_{2}$ into the cytoplasm of the MDCK II cells an increased level of the lipid integrated into plasma membrane is found. Golebiewska and coworkers injected $\mathrm{PIP}_{2}$ micelles into fibroblasts and found the injected lipid incorporating into the inner leaflet of the plasma membrane [163]. They report a significant lower diffusion coefficient for the injected lipid, which they attribute to a binding of lipids to the underlying cytoskeleton. The present study on the microinjection of $\mathrm{PIP}_{2}$ into MDCK II cells largely confirms the results from Golebiewska et al. by fluorescence microscopy, finding the lipid co-localized with the anchor-protein ezrin and the underlying cortical F-actin. These observations suggest a stronger binding of the cortex to the plasma membrane. Mechanical investigation after the microinjection of PIP $_{2}$ reveals a significant increase in membrane tension from 0.5 $\mathrm{mN} \mathrm{m}^{-1}$ by a factor of two. The elevated values for the membrane tension are attributed to an increased number of binding sites of the membrane to the cytoskeleton. The apparent area compressibility modulus rises more than 10 times compared to its initial value. This reflects a loss of excess membrane, leading to an inextensible plasma membrane. Confirming this view, AFM images reveal a loss of microvilli and a smoother surface. The loss of membrane reservoirs can be envisioned as follows. First, the increased number of binding sites of the membrane to the cytoskeleton reduces the available membrane reservoir. Second, by injecting the lipid $\mathrm{PIP}_{2}$ membrane traffic via endocytosis is enhanced [164] [165]. Membrane internalization would lead to reduced membrane area as observed for experiments with administration of MBCD, which in turn increases the apparent area compressibility modulus. The most striking observation is the regulation of the mechanical parameter $3 \mathrm{~h}$ after microinjection of the lipid. This indicates that the manipulated cell counteracts the membrane reduction and the associated increase in membrane tension. The plasma membrane of cells is a highly dynamic and strongly regulated structure. Many cellular processes like endo-and exocytosis [5] [6] and mitosis [4] are regulated by the membrane tension. Therefore, the cell has to ensure a certain set point of tension to guarantee ongoing membrane processes and to avoid lysis of the membrane..

In case of microinjecting neomycin, the high apoptotic rate of injected cell display the importance of a dynamic cellular cortex and the major role of $\mathrm{PIP}_{2}$ in the interplay between plasma membrane and cytoskeleton governing the mechanical properties of the membrane. 


\subsubsection{Injection of FITC-dextran - proof of principle}

Since the microinjection exhibit physiological stress, control experiments are necessary to elucidate the impact of the microinjection on the mechanical properties of the cells. Therefore, cells are microinjected with FITC-dextran (5 $\mathrm{mg} \mathrm{mL}^{-1}$ ). After $1 \mathrm{~h}$ of equilibration time, continuous force curves are taken to map the elastic response of the manipulated and adjacent cells. The impact of the injection is quantified with two mechanical approaches, the Hertzian theory and the extended liquid droplet model to obtain the mechanical parameters Young's modulus $E$, membrane tension $T_{0}$ and apparent area compressibility modulus $K_{A}$.
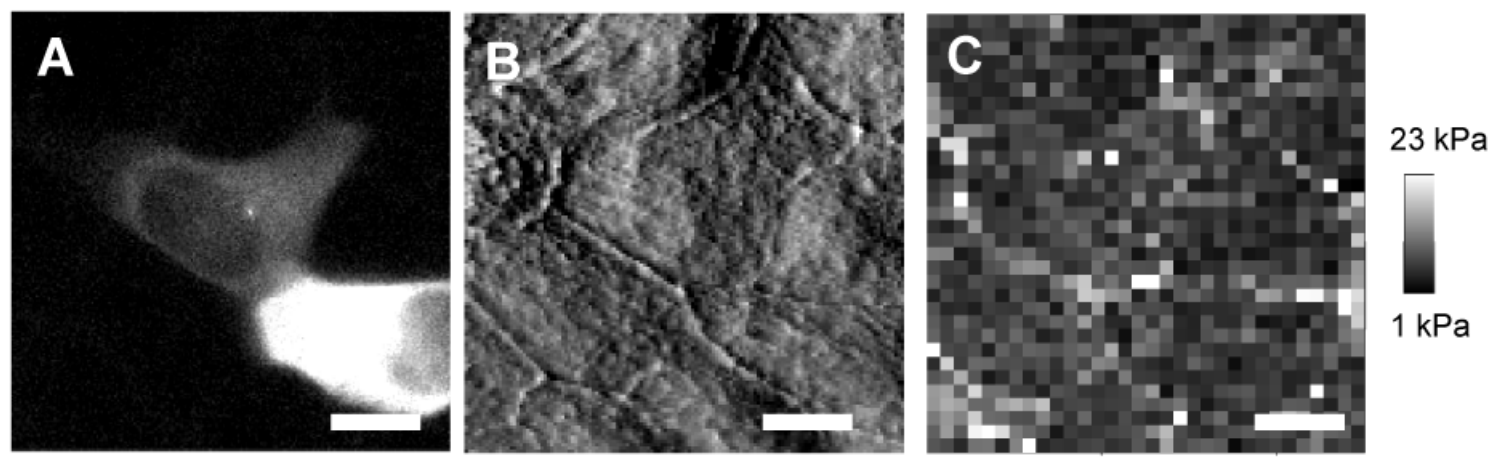

Figure 64: Microinjection of FITC-dextran into confluent MDCK II cells. A: Fluorescence micrograph of FITC-dextran injected cells. B: AFM-deflection image after injection. C: Young's modulus map after microinjection of FITC-dextran. Scale bar $10 \mu \mathrm{m}$.

The Young's modulus of injected cells compared to untreated adjacent cells display no significant difference $(\alpha$-value $=0.001$ for two-tailed Wilcoxon rank sum test) (Figure 65).
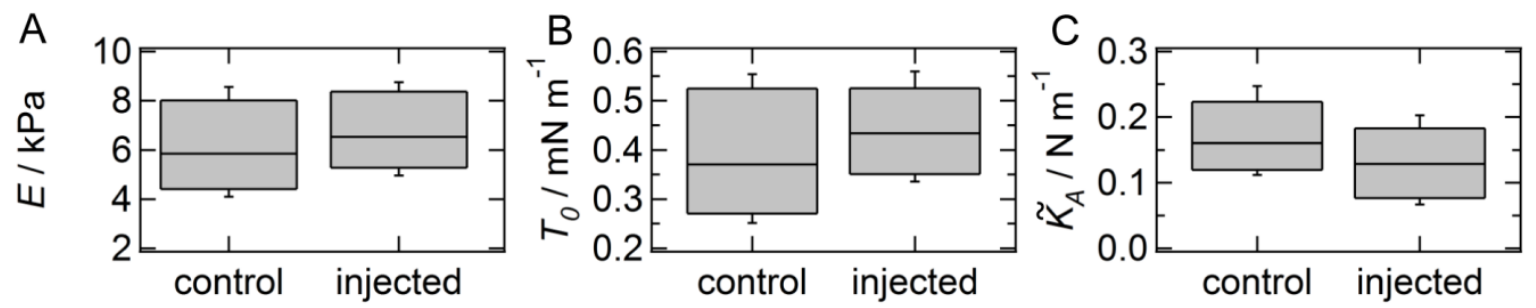

Figure 65: Evaluation of mechanical changes due to microinjection. A: Young's modules, B: Membrane tension, C: Apparent area compressibility modulus of FITC-dextran injected cells and untreated one.

The Young's modulus of untreated MDCK II cells is $6.6 \mathrm{kPa} \pm 3.5 \mathrm{kPa}$ and not change after injection of FITC-dextran $(6.9 \mathrm{kPa} \pm 2.5 \mathrm{kPa})$. By applying the extended liquid droplet model for adherent and confluent cells to the experimental data, the membrane tension $T_{0}$ and the area compressibility modulus are obtained. The manipulation of cells by 
microinjection shows a negligible change in the mechanical parameter. The membrane tension of untreated cells $\left(0.41 \mathrm{mN} \mathrm{m}^{-1} \pm 0.19 \mathrm{mN} \mathrm{m}^{-1}\right)$ not changes significantly after the microinjection $\left(0.45 \mathrm{mN} \mathrm{m}^{-1} \pm 0.15 \mathrm{mN} \mathrm{m}^{-1}\right)(\alpha$-value $=0.01$ for two-tailed Wilcoxon rank sum test). The apparent area compressibility modulus also reveals almost no change due to the microinjection. The value for untreated cells equals those after injection (before: 0.17 $\mathrm{N} \mathrm{m}^{-1} \pm 0.08 \mathrm{~N} \mathrm{~m}^{-1}$; after: $\left.0.15 \mathrm{~N} \mathrm{~m}^{-1} \pm 0.11 \mathrm{~N} \mathrm{~m}^{-1}\right)(\alpha$-value $=0.001$ for two-tailed Wilcoxon rank sum test).

This experiments show, that the microinjection itself exhibit no influence on the mechanical properties of the manipulated cells. This observation is important since all changes due to substance injection can be attributed to the substance itself and its impact on the cellular function. 


\subsection{Mechanics of Cell Membrane Fragments ${ }^{67}$}

So far, we attributed all changes of the mechanical properties to alterations directly associated with the plasma membrane itself excluding contributions from the cell interior such as cytosol, nucleus or cytoskeleton. Direct evidence that the plasma membrane is predominantly responsible for the mechanical response of living cells in a confluent monolayer can only be provided by probing the elasticity of isolated, planar apical cell membrane fragments. Previously, we established a protocol, the sandwich-cleavage approach that allows measuring the local elastic properties of isolated apical cell membrane sheets on porous supports (pore size $1.2 \mu \mathrm{m}$ ) (Figure $66 \mathrm{~A}-\mathrm{D})$ [166].

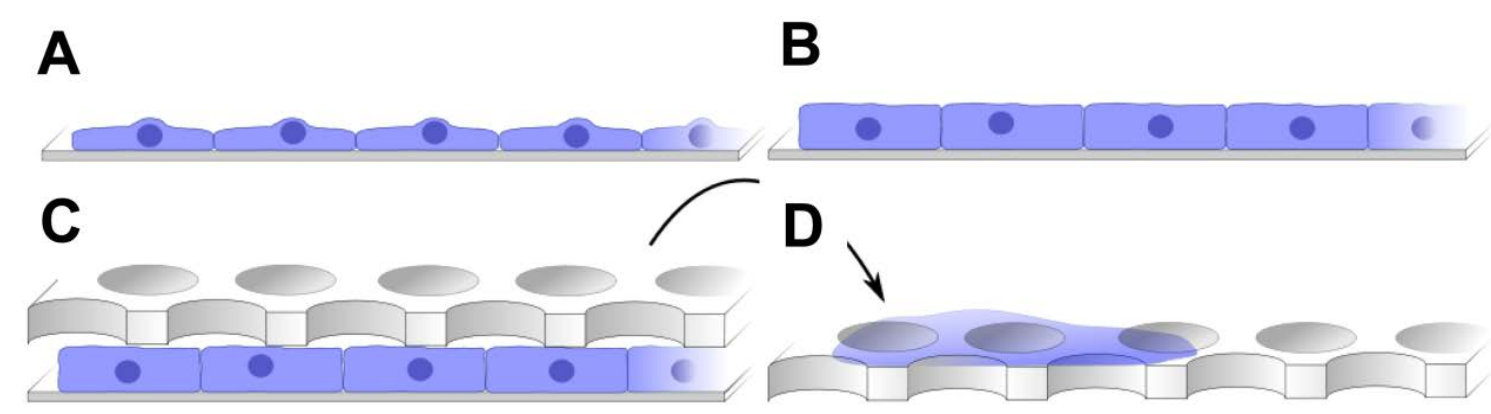

Figure 66: Illustration of apical membrane sheet preparation on porous substrate. A-B: Confluent MDCK II cells are hypoosmotically shocked prior to membrane deposition. C-D: A poly-D-lysin coated porous chip is gently pressed on the apical surface of the cells. By lifting the chip off the cells, the apical membrane is transferred to the porous substrate.

Site-specific force indentation curves in the center of each individual pore provide a map of membrane elasticity in the absence of contributions from the cell body and also largely without interference from cytoskeleton (Figure $67 \mathrm{~B}, \mathrm{D}$ ). The porous mesh allows to map the site-specific area compressibility modulus of apical cell membranes neglecting bending of the membranes for reasons that have been discussed previously [167]. Force indentation curves were subject to fitting a point-load force model that comprises a linear term

\footnotetext{
${ }^{6}$ Preparation of the membrane sheets on porous substrate and mechanical experiments are done by Dr.Tamir Fine [167].

${ }^{7}$ Parts of this chapter are published in Pietuch, A.; Brückner, B.R.; Fine, T.; Mey.I., Janshoff, A. (2013) Elastic properties of cells in the context of confluent cell monolayers: impact of tension and surface area regulation. Soft Matter, 2013, 9 (48), 11490 11502.
} 
associate with pre-stress of the membrane and a cubic term representing nonlinear membrane theory $[168,169]$.
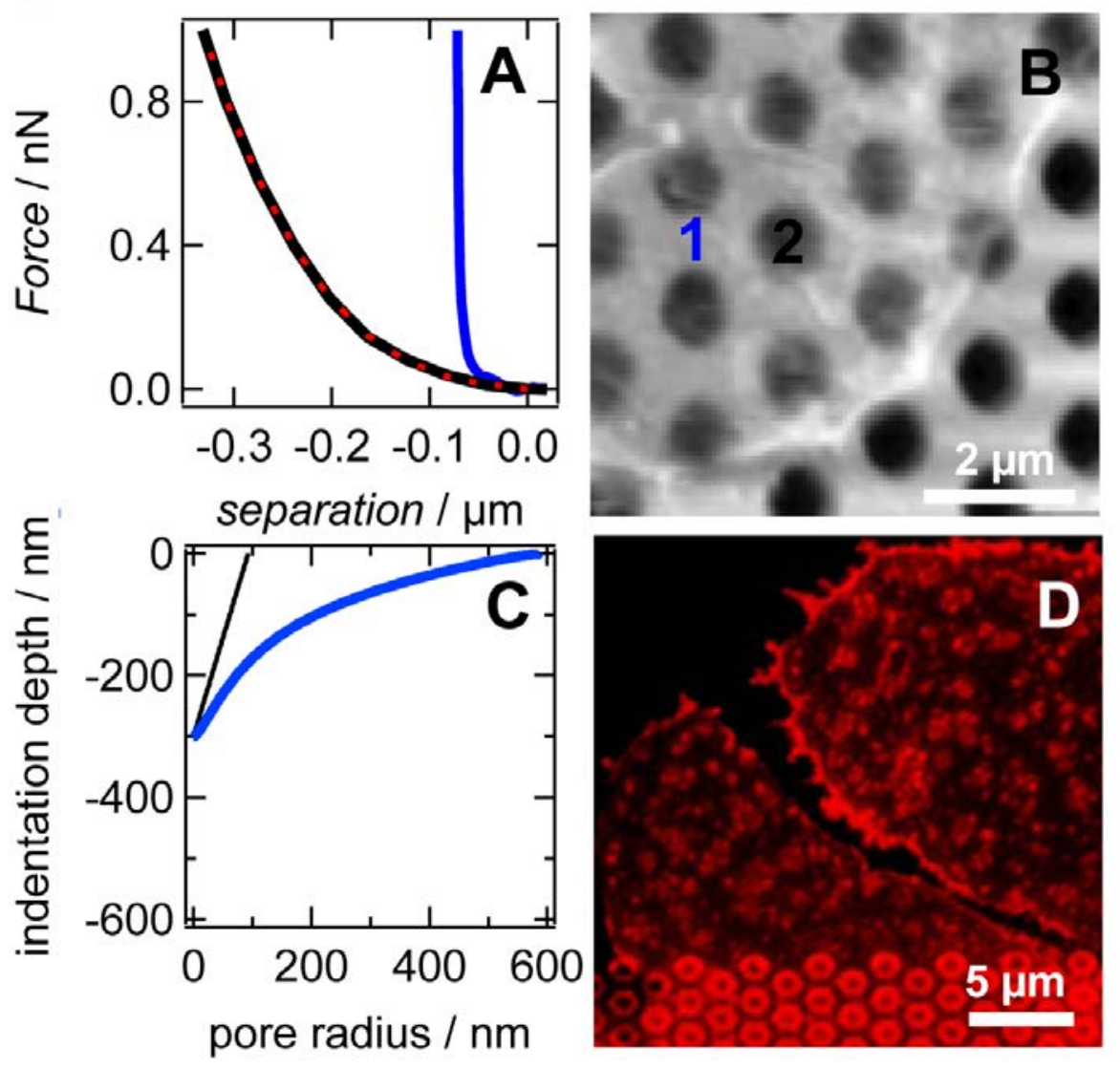

Figure 67: A: Force-distance approach curves taken on the rim (blue) or on a pore covered with membrane (black). B: AFM height images of apical membrane sheets on a porous substrate. C: Calculated shape (blue line) of a membrane indented by a conical or pyramidal indenter (black line) assuming a lateral tension $T_{0}$ of $0.16 \mathrm{mN} / \mathrm{m}$ and a bending modulus $\kappa$ of $25 k_{\mathrm{B}} T$. The shape of the membrane is essentially identical to that of a point-like indenter justifying the use of equation (35) to fit the indentation data. D: Fluorescence micrograph of apical membrane sheets labeled with $\operatorname{DiI}(18)$ on a porous substrate.

$F(h)=T_{0}(2 \pi R) \frac{h}{2 R}+\frac{\pi K_{A}}{3 R} h^{3}$

Assuming a point-load force is justified since the shape of the indented membrane is indistinguishable from that of pyramidal indenter (Figure $67 \mathrm{C}$ ). The tension $T_{0}$ can be mainly attributed to adhesion of the apical membrane fragment to the pore rims and is therefore not an intrinsic parameter of the cell membrane. Nevertheless, the values are close to those produced by the actomyosin cortex in living cells. $\tilde{K}_{A}$ denotes the apparent area compressibility modulus of the apical cell membrane. The model is essentially identical to the one proposed by Discher and coworkers but accounts for the altered geometry $(R=$ radius of the pore). Figure 67 A shows two force indentation curves, one 
obtained from the pore rim (blue, 1) displaying hard-wall repulsion and the other indentation curve recorded on the center of a pore covered with the apical membrane of confluent MDCK II cells (black, 2). The dotted red line represents a fit according to eq. 37. The corresponding AFM image is shown in Figure 67 B displaying no obvious sign of an actin terminal web. Fluorescence micrographs of apical cell membranes spanning the porous matrix show still the presence of protrusions probably originating from microvilli (Figure $67 \mathrm{D}$ ) but also show no sign of cytoskeleton filaments. We found a mean tension $T_{0}$ of $0.16 \pm 0.07 \mathrm{mN} \mathrm{m}^{-1}$ and apparent area compressibility modulus $\tilde{K}_{A}$ of $0.009 \pm 0.001$ $\mathrm{N} \mathrm{m}^{-1}$ representative for a plasma membrane lacking cytoskeleton filaments but associated to a lipid reservoir providing excess area upon stretching the planar membrane. The value corresponds well to the apparent $\tilde{K}_{A}$ values found for vital MDCK II cells (Figure 36 C) supporting the view that the plasma membrane largely governs the elastic response to a central load.

In conclusion, the ultimate proof that indeed the plasma membrane is responsible for the mechanical properties of confluent epithelial cells is given by probing isolated apical membrane sheets spanned over pores. The found values for the area compressibility modulus $\tilde{K}_{A}$ are essentially identical in comparison to living cells. This clearly demonstrates that the elastic response of the plasma membrane and not the properties of the underlying cortex are probed.

The mechanical approach of indenting cells with an AFM probe and applying the extended liquid droplet model for adhering and confluent cells enables the investigation of the plasma membrane as tension regulator during different cellular process. Although the elastic response of the membrane is mainly recorded during to these measurements, it is apparently the cytoskeleton which enables the plasma membrane to be a highly regulated and dynamic structure. 


\subsection{Osmotic Challenge to the Cellular Membrane ${ }^{8}$}

The plasma membrane of cells is a highly dynamic and strongly regulated two-dimensional liquid crystal. Many cellular processes like endo-and exocytosis [5] [6], cell migration [3], cell spreading $[8,9]$ and mitosis [4] are regulated by an intrinsic feature of the plasma membrane, the membrane tension. The plasma membrane tension encompasses the inplane tension of the lipid bilayer, the membrane-cytoskeleton adhesion, and is actively controlled by the contractile actomyosin cortex [10]. The intricate interplay between the plasma membrane and its cortex enables cells to withstand mechanical challenges posed by the environment. Considering that membranes are thin and fragile structures sophisticated feedback circuits are necessary based on tension homeostasis to maintain an intact shell. Osmotic stress is a physiologically relevant mechanical stimulus since animal cells have to sustain substantial fluctuations in the osmolarity of external fluids, which produces considerable pressure differences between the cytosol and the environment. Osmotic pressure forces the cell to quickly adapt in order to avoid damages of the inextensible plasma membrane. When cells are subjected to a hypoosmotic solution they increase their volume due to influx of water and subsequently their surface area. Groulx et al. reported, that animal cells can increase their surface area by a factor of 3 and ten times their volume, depending on the cell type [21]. As membranes cannot bear large strains (3-4\%), they require regulatory processes to maintain the overall plasma membrane tension below lysis tension, more precisely, tension driven surface area regulation is necessary to accommodate changes in tension. Concretely, high tension is buffered by an excess of membrane area, while a decrease in membrane area is triggered if the tension lowers [17]. To provide sufficient membrane area, cells store excess membrane in reservoirs like microvilli and caveolae, which by virtue of unfolding can buffer membrane tension. Kozera and coworkers showed that caveolae acting as membrane reserves compensate for an increase in membrane tension during stretching induced by swelling [20]. So far, a comprehensive picture of membrane homeostasis is lacking since it is difficult to measure surface area and tension simultaneously to confirm the regulative relationship between the two parameters. Polar epithelial cells as those from the intestine or kidney are suitable candidates to study the impact of environmental physicochemical stimuli on changes in

\footnotetext{
${ }^{8}$ Main parts of this chapter are published in Pietuch, A.; Brückner, B. R.; Janshoff, A. (2013) Membrane tension and homeostasis of epithelial cells through surface area regulation in response to osmotic stress. BBA - Molecular Cell Research 1833, 712-722.
} 
membrane tension since they frequently face changes in the chemical potential. Here, we used Madin Darby canine kidney cells (MDCK II) grown to confluence and challenged by different osmotic solutions to investigate the mechanical response of the plasma membrane with respect to tension-buffering membrane reservoirs. Therefore, indentation experiments, analyzed with an extend liquid droplet model for adherent cells, were carried out with an atomic force microscope (AFM) to simultaneously assess local changes in membrane tension and at the same time and location the excess membrane area as a function of osmotic pressure. The latter one is achieved by measuring the apparent area modulus of the plasma membrane mirroring the amount of stored excess area. Together with membrane tether pulling experiments, as an independent mechanical approach, we were able to show how epithelial cells adjust their surface area in response to tension changes. Furthermore, we monitored morphological changes due to cell swelling and shrinking showing distinct alteration in tension buffering membrane reservoirs (microvilli).

\subsubsection{Shape Changes during Osmotic Stress}

Our first task towards a comprehensive mechanical model that explains the interplay of membrane tension and surface area regulation was to examine the impact of osmotic stress on the morphology of the epithelial cell line MDCK II. Therefore, confluent cells are challenged for 30 min with solutions of different osmolarity and the overall morphological response of the apical surface is investigated by recording images with an atomic force microscope. The topographical images provide information about roughness of the plasma membrane, height and diameter of the cell, and presence of protrusions such as microvilli. Under isoosmolar conditions well-established microvilli are found and the visible cell shape resembles a spherical cap with a height of $1 \mu \mathrm{m}$ and radius of $10 \mu \mathrm{m}$ (median), respectively (Figure $68 \mathrm{~A}, \mathrm{E}, \mathrm{I}, \mathrm{J})$.

Under mild hypotonic stress (200 mOsm) the cell size increases but the plasma membrane surface still displays microvillar structure (Figure $68 \mathrm{~B}$ ). While the radius of the cells is conserved, the height of the spherical cap increases from $1 \mu \mathrm{m}$ to $1.7 \mu \mathrm{m}$ (median) demonstrating a swelling of the cell. The most prominent change in surface structure and cell height is visible for cells challenged with a strong hypotonic solution (80 mOsm) (Figure $68 \mathrm{C}$ ). Microvilli are largely removed generating a smooth membrane surface (Figure 68 G, F). The height of the highly stressed cells increases up to $2.8 \mu \mathrm{m}$ (height of 
the spherical cap) (Figure 68 I). We also investigated the impact of hypertonic stress on cellular morphology. Although the topographical image shows no significant effect of the treatment (Figure $68 \mathrm{H}$ ), we observe changes in cell height and radius (Figure 68 D). While hypoosmotic stress leads to a cell swelling, the inverse treatment leads to a shrinking of the cells to a height of merely $0.6 \mu \mathrm{m}$ and a radius of $7 \mu \mathrm{m}$ (median) (Figure $68 \mathrm{I}, \mathrm{J})$. Analyzing the cellular shape and surface structure after application of osmotic stress enables us to determine important geometrical input parameters for our mechanical model (vide infra)
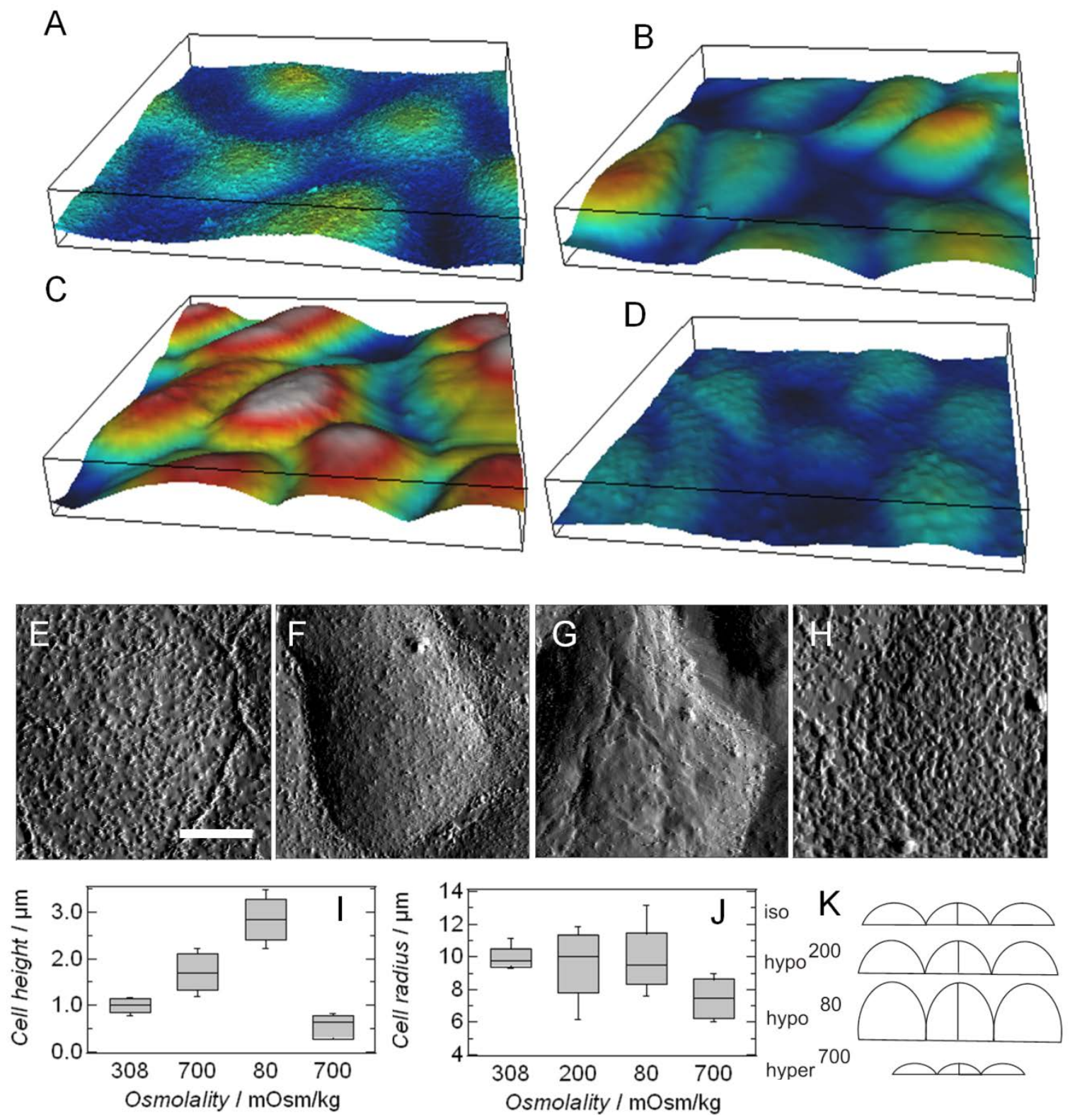

Figure 68: Morphological changes of confluent MDCK II cell after osmotic stress. A-D 3D-height images of confluent MDCK II cells treated with different osmotic solutions. A: isotonic. B: 200 mOsm. C: 80 mOsm. 
D: 700 mOsm. Height of the Box is $5 \mu \mathrm{m}$, box length and width is $50 \mu \mathrm{m}$. E-H: AFM deflection images, scale bar is $10 \mu \mathrm{m}$. E: untreated cell. F: $200 \mathrm{mOsm}$. G: 80 mOsm. H: 700 mOsm. I: Cell height and J: Cell radius, extracted from AFM height images, at least $n=10$ cells. K: Schematic drawing of spherical caps to demonstrate change in cell size due to osmotic treatment.

A spherical cap essentially captures the general shape of the apical part of cells in a confluent monolayer in the most simplistic way (Figure $68 \mathrm{~K}$ ). The cap is characterized by a contact angle and radius at the base (or alternatively the height of the cap), which are crucial and sufficient to describe the shape of the cell upon indentation according to the liquid droplet theory of Sen et al. [25].

Values displayed in Table 11 are used to model the spherical cap for different osmotic treatments.

Table 11: Parameters for modeling spherical cap describing confluent MDCK II cells when subjected to osmolar stress.

\begin{tabular}{ccccc}
\hline & $\begin{array}{c}\text { isotonic 300 } \\
\text { mOsm }\end{array}$ & $\begin{array}{c}\text { hypoosmotic } \\
\text { 200 mOsm }\end{array}$ & $\begin{array}{c}\text { hypotonic } \\
80 \text { mOsm }\end{array}$ & $\begin{array}{c}\text { hypertonic } \\
700 \text { mOsm }\end{array}$ \\
\hline$\phi_{0}\left[^{\circ}\right]$ & 20 & 23 & 30 & 17 \\
$R_{0}[\mu \mathrm{m}]$ & 35 & 30 & 26 & 28 \\
\hline
\end{tabular}




\subsubsection{Mechanical Response to Hypoosmotic Stress}

Cells swell under hypoosmotic conditions and thereby deform and generate hydrostatic pressure against the plasma membrane. This effect is investigated by indentation as well tether pulling experiments using force microscopy carried out with an AFM.

Regarding the experimental data without applying a mechanical model, the difference in the elastic response of treated cells became obvious. Force distance curves taken on osmotic stressed cells display a change in the slope (Figure 69 A). While a mild hypoosmotic solution (200 mOsm) shows almost no change in the shape of the approach curve, a lowering of the external osmolarity to 80 mOsm leads to a larger indentation depth at constant force, clearly indicative of a softening of the cells assembly mirrored in reduced values for $T_{0}$ and $\tilde{K}_{A}$. In fact, exposing cells to a strong hypotonic solution (80 mOsm) results in the same degree of cell softening as obtained from experiments where the F-actin cytoskeleton is destroyed by administration of cytochalasin D. This suggests that during hypoosmotic stress the deformation and outward bulging of the plasma membrane induced by the large internal pressure could lead to a reorganization and destruction of the cytoskeleton in response to the external force.

By applying the extended liquid droplet model for adhering cells, indentation experiments yielding force distance curves (Figure 69 A) allows calculating mechanical parameter like the apparent area compressibility modulus $\tilde{K}_{A}$, reflecting changes in effective membrane area and the membrane tension $T_{0}$. The mechanical parameters display for the mild hypoosmotic stress a significant increase in tension $T_{0}\left(0.35 \pm 0.1 \mathrm{mN} \mathrm{m}^{-1}\right.$, median; isotonic $0.29 \pm 0.09 \mathrm{mN} \mathrm{m}^{-1}$, median) and apparent area compressibility modulus $\tilde{K}_{A}(0.06 \pm 0.09$ $\mathrm{N} \mathrm{m}^{-1}$, median; isotonic $0.03 \pm 0.08 \mathrm{~N} \mathrm{~m}^{-1}$ ). The reduction in excess membrane area is due to cell swelling, smoothing out membrane reservoirs as microvilli [20].

Cell swelling exerts pressure across the plasma membrane and inevitably increases the membrane's in-plane tension. The cell is not able to quickly reduce tension by providing excess area stored in protrusions and invaginations of the plasma membrane to counterbalance the rising tension since the increased cell volume diminishes this excess membrane. 
A

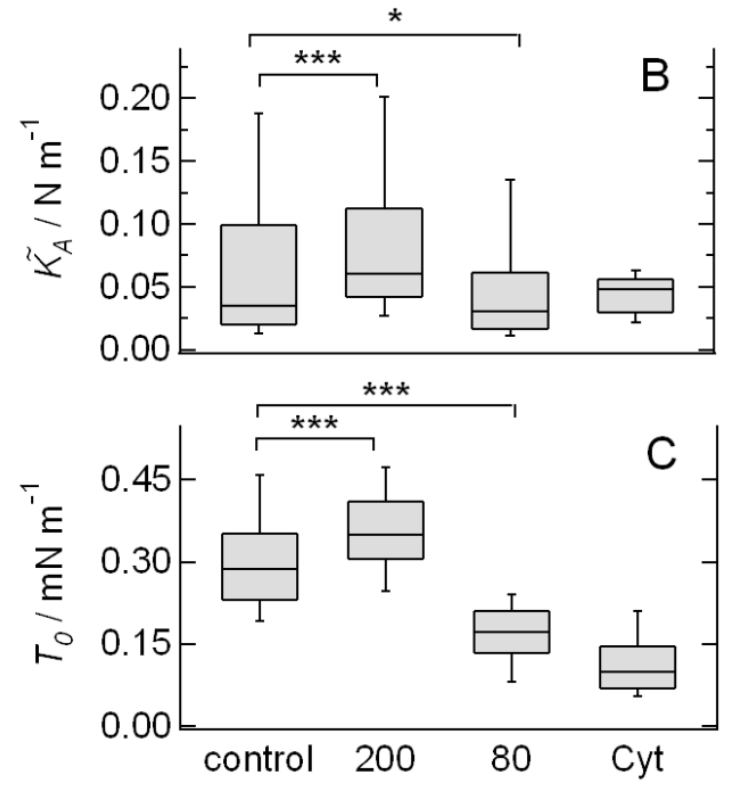

Figure 69: Indentation experiments revealing mechanical information of hypoosmotic stressed confluent MDCK II cells. A: Representative force distance traces taken on treated cells. Trace curve is used for determine the area compressibility module and tension $T_{0}$ using a modified liquid droplet model for indentation experiments fitted to the experimental data. B: Area compressibility modulus, reflecting the changes in membrane area during the treatment. C: Changes in membrane tension $T_{0}$ by altering the osmotic stress or F-actin integrity by cytochalasin D.

In order to obtain a more comprehensive picture of the destructive nature of hypoosmotic stress the structural changes of the F-actin cytoskeleton is investigated by fluorescence staining. Figure 71 shows how the F-actin rearranges as a function of osmolarity. The fluorescence micrographs display no change in the structure of the cortical F-actin at the apical part of the cells after treatment with mild hypotonic solution (Figure 71 B1). In the basolateral region, however, an alteration in the formation of stress fibers is observed (Figure $71 \mathrm{~B} 2$ ). Comparing with an untreated sample, the structure of the stress fibers is less pronounced.

Membrane tension contains contribution from membrane-cytoskeleton-adhesion as well as tension in the bilayer itself [124]. In general, tension values obtained from tether pulling are very similar to those assessed by indentation experiments largely confirming the assumed mechanical model to describe indentation experiments. Interestingly, however, mild hypotonic conditions produce a decrease in membrane tension $T_{t}$ determined from tether pulling in contrast to the tension $T_{0}$ obtained from indentation treatment $\left(T_{t}\right.$ (200 
mOsm) $=0.17 \pm 0.11 \mathrm{mN} \mathrm{m}^{-1}$, median; $T_{t}$ ( isotonic, $\left.300 \mathrm{mOsm}\right)=0.25 \pm 0.13 \mathrm{mN} \mathrm{m}^{-1}$, median).

Increasing the swelling process by using a strong hypoosmotic solution leads to a change in the apparent area compressibility modulus, in particular the loss of the higher values, which are attributed to regions on the cell surface with direct connection to the underlying cytoskeleton. At the same time, membrane tension $T_{0}$ decreases to a value of $0.17 \pm 0.05$ $\mathrm{mN} \mathrm{m}^{-1}$ (median) (Figure $69 \mathrm{C}$ ). Although the cells increase their volume by swelling this leads to a softening of the cells. The F-actin cytoskeleton shows a dramatic reorganization by strong osmotic challenge (80 mOsm). In the apical region, no microvilli or cortical structures are visible in the fluorescence micrographs. Stress fibers are completely degraded (Figure 71). Concomitantly tether forces are shifted to lower values (Figure 71), resulting from a decreased membrane tension $\left(0.14 \pm 0.12 \mathrm{mN} \mathrm{m}^{-1}\right.$, Figure 70). A similar disassembly of the cortical cytoskeleton and stress fibers can be achieved by treating the cell with cytochalasin $\mathrm{D}$. This leads to a decreased membrane tension, measured by indentation and tether pulling (Figure 69 A, Figure 70 and Figure 71 D).

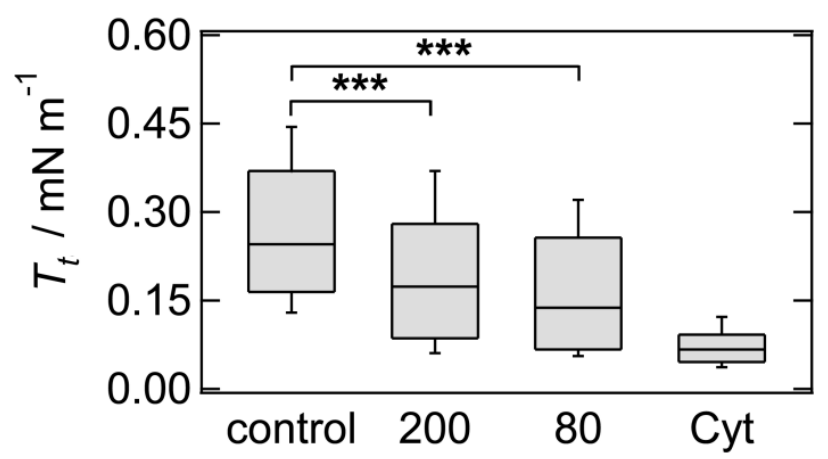

Figure 70: Membrane Tension $T_{t}$ calculated from tether pulling experiments. For computing $T_{t}$ a bending module $\kappa$ of $10^{-19} \mathrm{~J}$ representing fluid lipid bilayer is assumed. Viscous contributions to the tether force are neglected as a first approximation $[110,170]$.

Destruction of the F-actin cytoskeleton with a drug as cytochalasin D displays a similar change of the relevant mechanical parameters such as tension and area compressibility as hypoosmotic stress, although the cells are increasing their volume due to swelling. This leads us to the assumption that the cell counteracts the membrane deformation and increased membrane tension induced by osmotic swelling due to hypoosmotic stress by disassembly of the cytoskeleton to release tension. This release of tension avoids plasma membrane lysis, which inevitably would destroy the cell. 


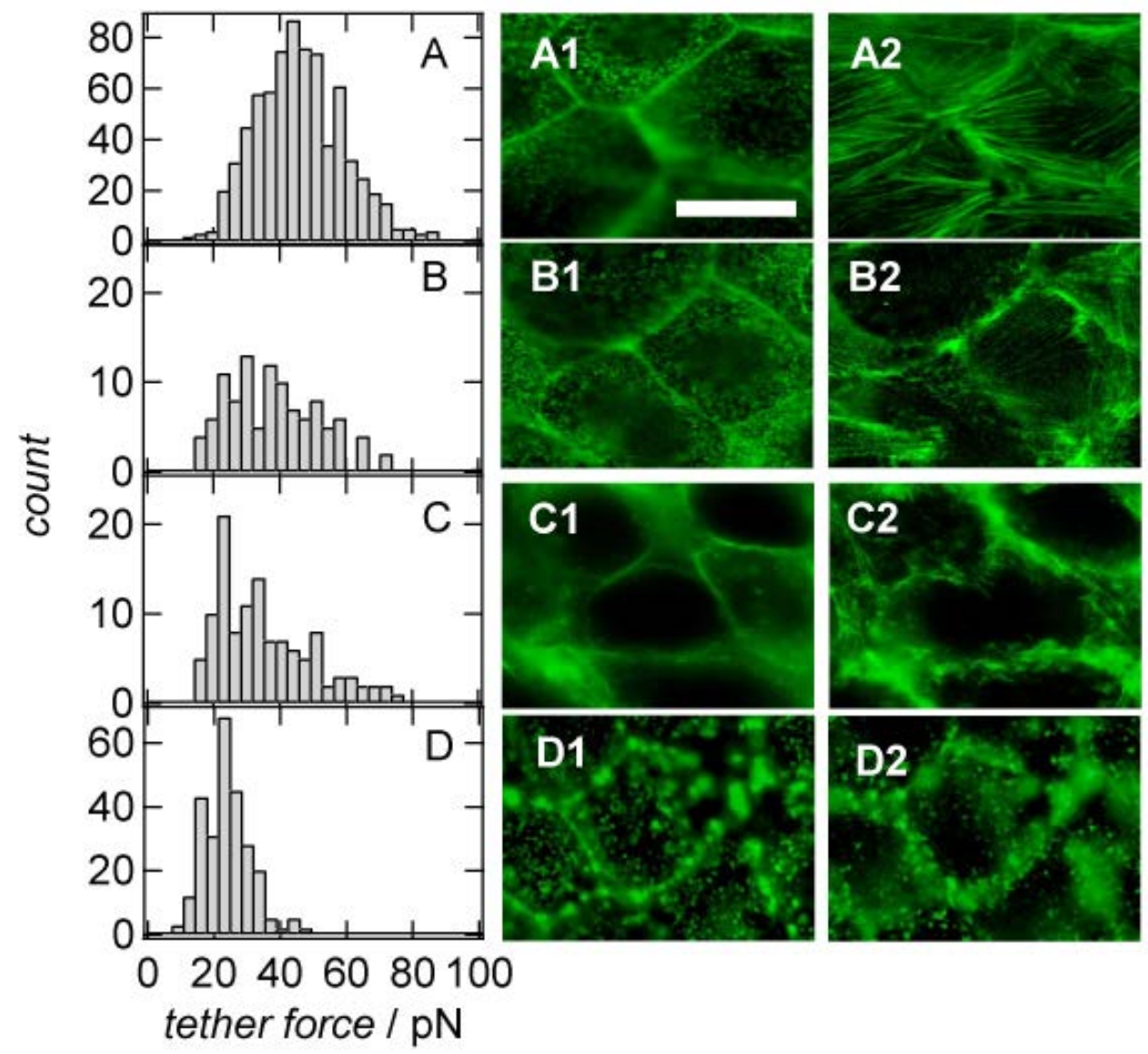

Figure 71: Histogram of tether forces obtained from retrace curves of a force cycle of tether pulling experiments, with corresponding fluorescence micrographs of different treated MDCK II cell. A: Histogram of tether forces of untreated cells (isotonic, $308 \mathrm{mOsm}$ ). A1: shows apical part of the cell, A2 the corresponding basolateral part. B: 200 mOsm. C: 80 mOsm. D: Cells treated with F-actin destabilizing cytochalasin. Scale bar is $20 \mu \mathrm{m}$.

\subsubsection{Cells Regulate Membrane Tension/Area during Prolonged Hypoosmotic Challenge}

Plasma membrane tension drops due to strong hypoosmotic stress, therefore it is proposed that deformation of the membrane, a result from cell swelling, reorganizes the F-actin cytoskeleton to counteract the generated force [6]. When membrane tension drops the plasma membrane area is reduced by endocytosis [171-173]. But this active volume regulation was not observed in the above described experiments during 30 min test period. Hence the observation period is extended. The mechanical properties of a confluent MDCK II monolayer are investigated during strong hypoosmotic stress over 6 hours (Figure 72). The apparent compressibility modulus $\tilde{K}_{A}$, reflecting the change in membrane area, increases during the observation time (Figure $72 \mathrm{~A}$ ). While the value for untreated 
cell (isotonic) lies in the range of $0.03 \pm 0.08 \mathrm{~N} \mathrm{~m}^{-1}$ (median), the value for 6h osmotic challenge rises to $0.15 \pm 0.07 \mathrm{~N} \mathrm{~m}^{-1}$ (median).
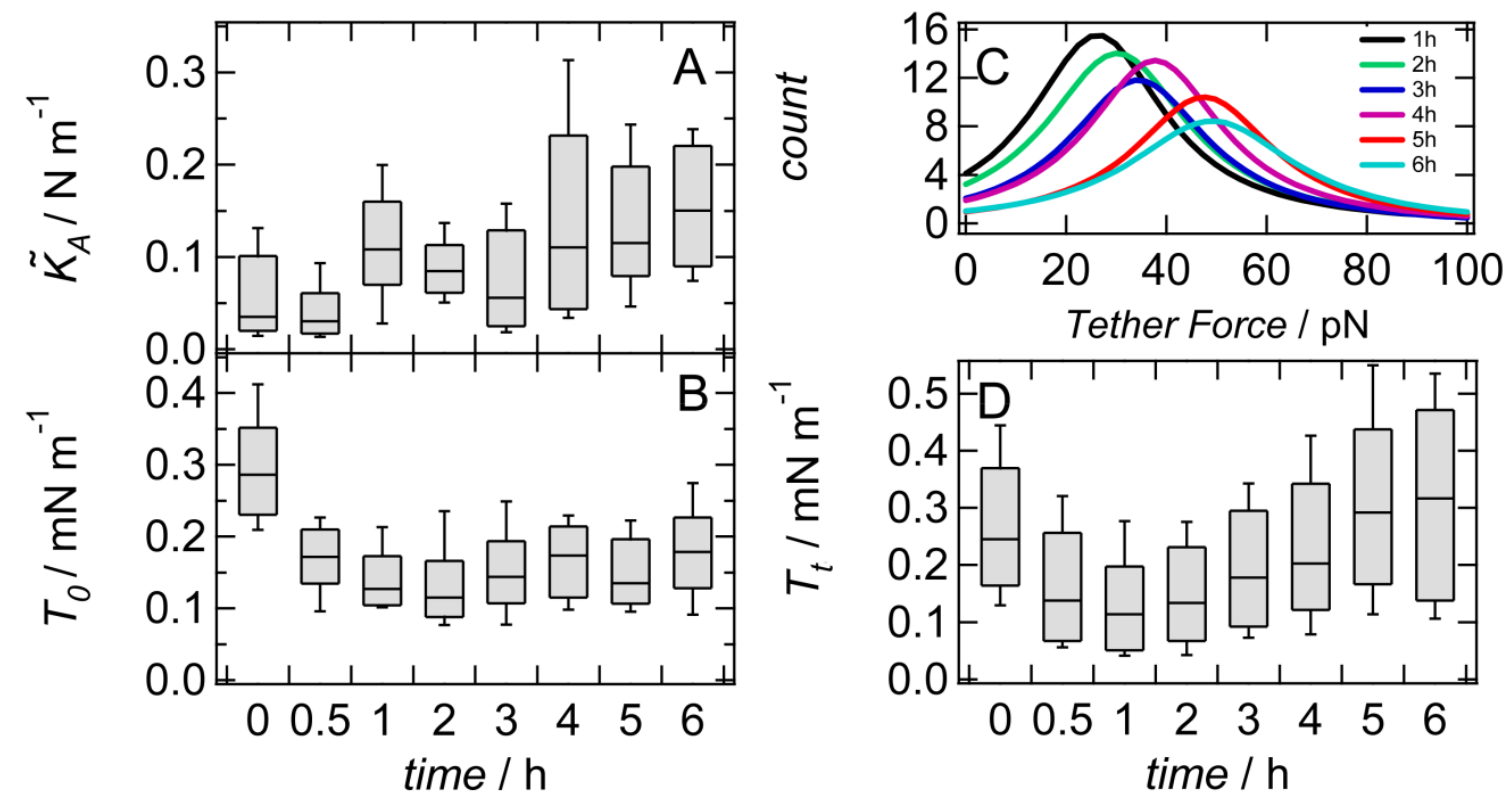

Figure 72: Mechanical changes resulting from long incubation in hypoosmotic solution. A: Apparent area compressibility modulus at different time points. B: Membrane tension obtained from indentation experiments. C: Lorenz-Fits of tether force histogram displaying change in tension during regulatory process. D: Membrane Tension $T_{t}$ calculated from tether pulling experiments.

For untreated cells the $\tilde{K}_{A}$-value reflects a membrane folding factor of 6 , when suggesting an area compressibility modulus of $0.17 \mathrm{~N} \mathrm{~m}^{-1}$ as obtained from Eq. 7, emphasizing membrane reservoirs. During swelling the apparent area compressibility modulus is not changing (0.5h, $0.03 \pm 0.06 \mathrm{~N} \mathrm{~m}^{-1}$ (median)), while membrane tension ( $T_{0}$ and $\left.T_{t}\right)$ decreases, indicating a change in the cytoskeleton architecture. After 1h of incubation time the cell starts to regulate the membrane area, in particular to decrease it (Figure $72 \mathrm{~A}$ ) (Table 12). A higher endocytosis rate is found as a response to $2 \mathrm{~h}$ of strong hypoosmotic stress (Figure 73), i.e. a larger number of vesicles accumulating in the cytosol. 


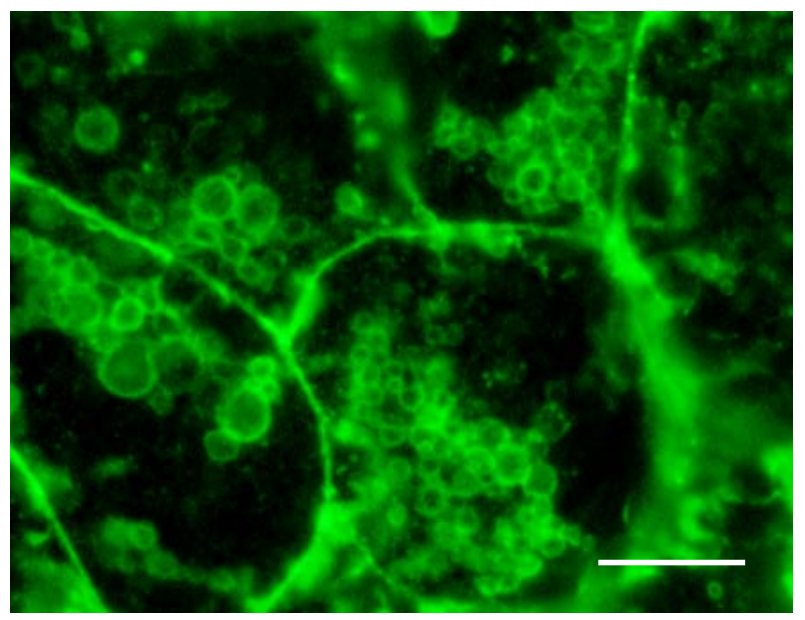

Figure 73: Fluorescence micrograph of confluent MDCK II cells challenged $2 \mathrm{~h}$ in strong hypoosmotic solution (80 mOsm). F-actin is labeled in green. Scale bar $10 \mu \mathrm{m}$.

At this time point, membrane tension estimated from tether pulling experiments increases (Figure 72 D) (Table 12). As shown in Figure $72 \mathrm{C}$ during 6 hours of incubation tether forces shifted to higher values comparable to values obtained from untreated cells (Figure $71 \mathrm{~A}$ ). It should be noted that both values $\tilde{K}_{A}$ and $T_{t}$ increases indicating a plasma membrane regulatory effect. While the tension $T_{t}$ from tether pulling experiments increases $T_{0}$ shows over the whole incubation time a slight increase up to value of about $0.18 \pm 0.06$ $\mathrm{mN} \mathrm{m}^{-1}$, but not reaching the value of untreated cells. Tether force mainly contributing from membrane adhesion to the cytoskeleton elevates when membrane tension is increased either by loss of membrane area due to internalization or binding the membrane via molecular-anchors to the underlying cortex.

To examine this, ezrin a member of the ERM family and F-actin are fluorescently labeled and visualized on the same spot. Structural organization and stabilization of the plasma membrane protrusions relies heavily on protein linkers such as ezrin connecting the cellular membrane to microfilaments [11, 15]. A change in the ezrin and F-actin distribution is observe during hypoosmotic stress (Figure 74). After $2 \mathrm{~h}$ of incubation the density and distribution of the protein ezrin is decreased in the apical region (Figure 74 B1). Concomitantly the cortical F-actin structure is altered. In the lower part of the cell, where the isotonic sample exhibits stress fibers (Figure 74 A4), the hypoosmotic stressed cell display a disassembled F-actin structure. This is not changing during the $6 \mathrm{~h}$ of observation time (Figure $74 \mathrm{C} 4$ ), where the basal F-actin structure equals the one after $2 \mathrm{~h}$. 
However, the apical structure after 6h (ezrin and F-actin distribution) shows a similar composition than the control cells (isotonic).
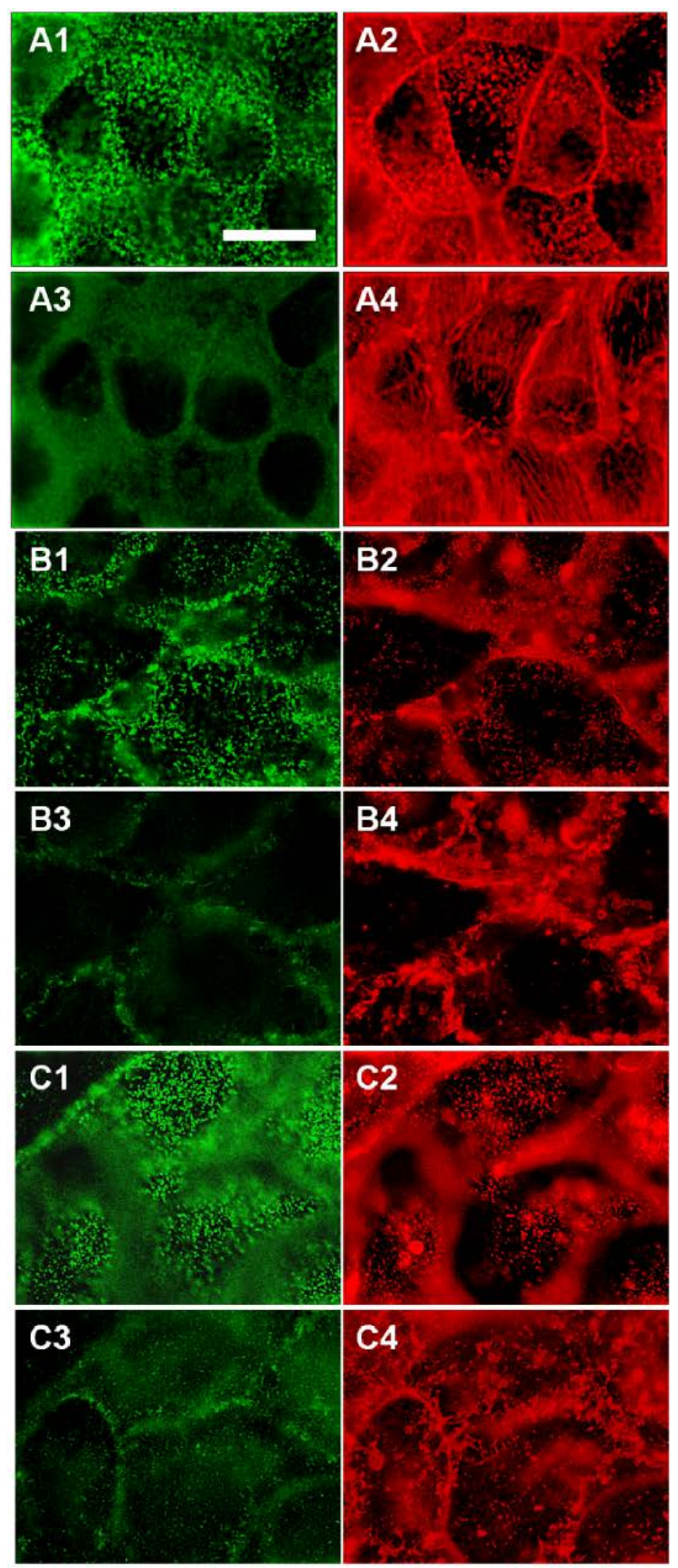

Figure 74: Fluorescence micrographs of confluent MDCK II cells challenged for $0 \mathrm{~h}(\mathrm{~A}), 2 \mathrm{~h}(\mathrm{~B})$ and $6 \mathrm{~h}$ (C) in strong hypoosmotic solution (80 mOsm). Microfilament- membrane anchor protein ezrin is labeled in green, F-actin in red. Scale bar is 20 $\mu \mathrm{m}$. 
Taken together, the apical structure reassembles but the basolateral one not. This is maybe an explanation for the different membrane tension obtained from indentation and tether pulling. While tension estimated from tethers $\left(T_{t}\right)$ contributes from membranecytoskeleton-adhesion and therefore increases over time, tension obtained from indentation ( $T_{0}$ ) mainly depends on the intactness of the underlying cytoskeleton, which is altered and shows a lower elasticity.

Summarizing, strong hypoosmotic stress for 30 min leads to a swelling of the MDCK II cells accompanied with a decrease in membrane tension $\left(T_{0}\right.$ and $\left.T_{t}\right)$ and a dramatic change in the F-actin structure. Longer treatment of MDCK II cells (6h) shows a stepwise increasing in the apparent area compressibility modulus and membrane tension $T_{t}$, displaying a regulatory effect.

Table 12: Mechanical parameter obtained from long incubation in hypoosmotic solution. Values are representing the median from boxplots in Figure 72 and the median absolute deviation.

\begin{tabular}{|c|c|c|c|c|c|c|c|c|}
\hline $\begin{array}{c}\text { Incubation time } \\
{[\mathrm{h}]}\end{array}$ & 0 & 0.5 & 1 & 2 & 3 & 4 & 5 & 6 \\
\hline$\tilde{K}_{A}\left[\mathrm{Nm}^{-1}\right]$ & $\begin{array}{c}0.03 \\
\pm 0.08\end{array}$ & $\begin{array}{c}0.03 \\
\pm 0.06\end{array}$ & $\begin{array}{c}0.11 \\
\pm 0.06\end{array}$ & $\begin{array}{c}0.08 \\
\pm 0.02\end{array}$ & $\begin{array}{c}0.06 \\
\pm 0.06\end{array}$ & $\begin{array}{c}0.11 \\
\pm 0.11\end{array}$ & $\begin{array}{c}0.12 \\
\pm 0.07\end{array}$ & $\begin{array}{c}0.15 \\
\pm 0.07\end{array}$ \\
\hline$T_{0}\left[\mathrm{mNm}^{-1}\right]$ & $\begin{array}{c}0.29 \\
\pm 0.09\end{array}$ & $\begin{array}{c}0.17 \\
\pm 0.06\end{array}$ & $\begin{array}{c}0.13 \\
\pm 0.04\end{array}$ & $\begin{array}{c}0.12 \\
\pm 0.06\end{array}$ & $\begin{array}{c}0.14 \\
\pm 0.06\end{array}$ & $\begin{array}{c}0.17 \\
\pm 0.05\end{array}$ & $\begin{array}{c}0.14 \\
\pm 0.06\end{array}$ & $\begin{array}{c}0.18 \\
\pm 0.06\end{array}$ \\
\hline$T_{t}\left[\mathrm{mNm}^{-1}\right]$ & $\begin{array}{c}0.25 \\
\pm 0.13\end{array}$ & $\begin{array}{c}0.14 \\
\pm 0.12\end{array}$ & $\begin{array}{l}0.11 \\
\pm 0.1\end{array}$ & $\begin{array}{c}0.13 \\
\pm 0.09\end{array}$ & $\begin{array}{c}0.18 \\
\pm 0.09\end{array}$ & $\begin{array}{c}0.2 \\
\pm 0.13\end{array}$ & $\begin{array}{c}0.29 \\
\pm 0.13\end{array}$ & $\begin{array}{c}0.32 \\
\pm 0.15\end{array}$ \\
\hline
\end{tabular}

Table 13: Parameters for modeling spherical cap describing confluent MDCK II cells, when subjected to hypotonic stress (80 mOsm) over $6 \mathrm{~h}$.

\begin{tabular}{ccccccc}
\hline & $1 \mathrm{~h}$ & $2 \mathrm{~h}$ & $3 \mathrm{~h}$ & $4 \mathrm{~h}$ & $5 \mathrm{~h}$ & $6 \mathrm{~h}$ \\
\hline$\phi_{0}\left[^{\circ}\right]$ & 30 & 30 & 30 & 25 & 25 & 25 \\
$R_{0}[\mu \mathrm{m}]$ & 26 & 26 & 26 & 38 & 38 & 38 \\
\hline
\end{tabular}




\subsubsection{Hyperosmotic Shock: From Liquid Droplet to a Drum like Geometry}

So far, we dealt with the mechanical response of cells in a confluent monolayer to exposure to hypotonic solutions. But what happens, if the cell condenses due to hypertonic stress? Will the excess supply of membrane area lead to a sudden drop in tension? Morphological changes are described during a hypertonic shock in Figure 68. In essence, we found that the cells shrink in height and diameter. This extreme shape change towards a more two dimensional drum-like apical side has an important impact on the determination of mechanical parameters obtained from indentation as well as tether pulling experiments. In Figure 69 we displayed force distance traces taken on osmotic challenged cells. Cells stressed with hyperosmotic solution, show the steepest slope, indicating a rigid or even solid-like structure. Concomitantly tether forces are shifted to higher values compared to control cells (Figure 75).
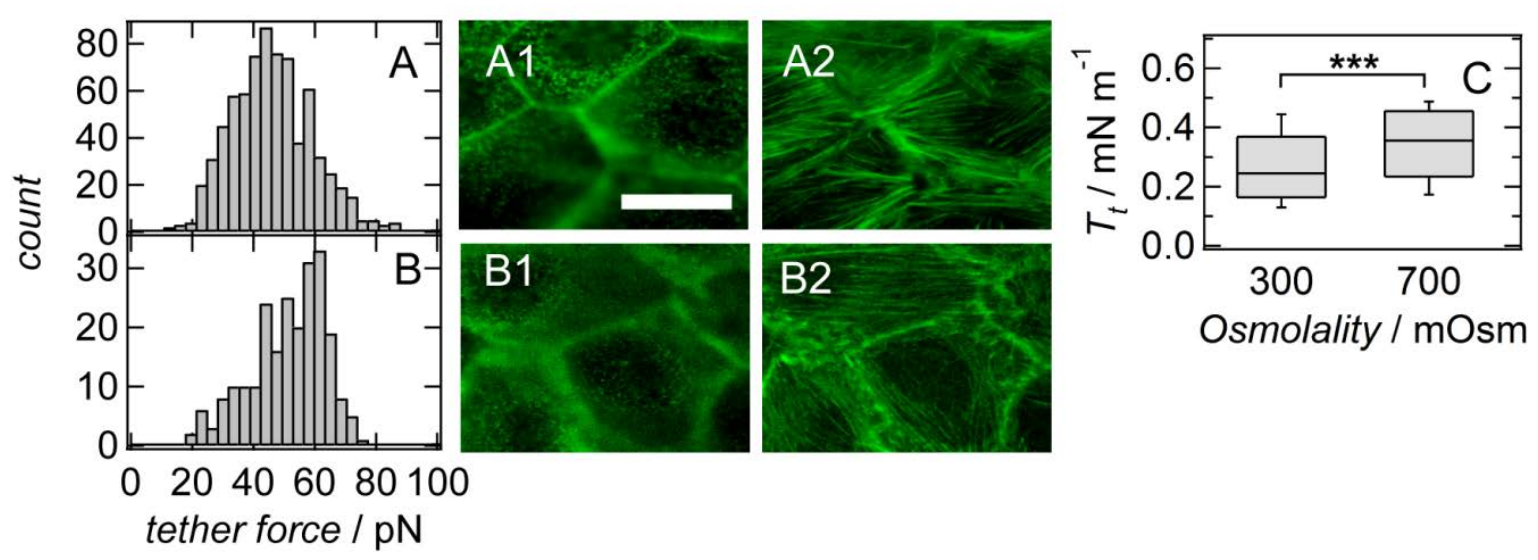

Figure 75: A: Histograms of tether forces obtained from experiments on hypertonic challenged cells compared to a control (700 mOsm 308 mOsm). Fluorescence micrographs of F-actin labeled MDCK II cells treated with isotonic (A1: apical, A2: basal) and hypertonic (B1: apical, B2: basal) solution for 30 min. Scale bar is $20 \mu \mathrm{m}$. C: Membrane tension $T_{t}$ estimated from tether pulling experiments.

Estimating membrane tension from tether forces we found an increase from $0.25 \pm 0.13$ $\mathrm{mN} \mathrm{m}^{-1}$ (median) to $0.36 \pm 0.13 \mathrm{mN} \mathrm{m}^{-1}$ (median) (Figure $75 \mathrm{C}$ ). Because of the extreme shrinkage of the cell by a factor of almost 2 (Figure 68), the cells are not anymore acting as a liquid droplet in spherical geometry. Mechanical parameters obtained from fitting the liquid droplet model to the experimental data are shown in Table 14. In particular the apparent area compressibility modulus is too high regarding a cellular membrane. Assuming a drum-like architecture which is essentially described by a 2-D planar circular 
membrane indented in the center by a point load force we can apply the following equation derived by Komaragir et al. [168] [169]. The first term is linear in penetration depth $h$ and dominated by pre-stress (tension) $T_{0}$ in the membrane. The second term captures lateral stretching of the membrane and solely depends on the membrane's area compressibility modulus $K_{\mathrm{A}}$. The radius of the circular membrane framed by the tight junctions of the adjacent cells is denoted by $R_{c}$.

The values for $\tilde{K}_{A}$ obtained from fitting the parameters of equation 37 to the force indentation curves increase upon exposure to hyperosmotic stress indicative of reduction of surface area, while $T_{0}$ decreases (table 14).

$F(h)=T_{0}\left(2 \pi R_{c}\right) \frac{h}{2 R_{c}}+\frac{\pi K_{A}}{3 R_{C}} h^{3}$

This would indicate that the substantial changes in the force indentation curve are mainly due to changes in cell morphology, i.e. geometry and not so much due to changes in elastic properties. This finding is corroborated by tether pulling experiments. In tether pulling experiments, the distribution of tension values slightly shifts to higher values upon exposure to hypertonic solutions. Estimating the apparent area compressibility modulus from tether experiments as it described by Equation 8 (section 3.2), gives a value of $0.21 \pm$ $0.09 \mathrm{~N} \mathrm{~m}^{-1}$, which is similar to $\tilde{K}_{A}$ found using Equation 35 (Table 14). The following scenario is conceivable: Tension of the bilayer drops due to osmotic pressure changes and the membrane collapses on the cytoskeleton. In turn, excess membrane area is removed by endocytosis and tension largely restored since the remaining osmotic pressure forces the membrane onto the cortex producing larger tether forces.

Table 14: Mechanical parameters obtained from hypertonic stress on confluent MDCK II cells.

\begin{tabular}{ccccccc}
\hline & $\begin{array}{c}\tilde{K}_{A} \text { Eq. 37 } \\
{\left[\mathrm{N} \mathrm{m}^{-1}\right]}\end{array}$ & $\begin{array}{c}\tilde{K}_{A} \\
\text { Discher }\end{array}$ & $\begin{array}{c}\text { radius } \\
{[\mu \mathrm{m}]}\end{array}$ & $\begin{array}{c}T_{0} \\
\text { Discher }\end{array}$ & $\begin{array}{c}T_{0} \text { Eq. 37 } \\
{\left[\mathrm{mN} \mathrm{m}^{-1}\right]}\end{array}$ & $\begin{array}{c}T_{t} \\
{\left[\mathrm{mN} \mathrm{m}^{-1}\right]}\end{array}$ \\
\hline hypertonic & $0.29 \pm 0.21$ & $2.52 \pm 1.13$ & $7.7 \pm 1.35$ & $0.31 \pm 0.65$ & $0.11 \pm 0.09$ & $0.35 \pm 0.17$ \\
\hline
\end{tabular}




\subsubsection{Cellular Reaction on Distilled Water}

The strongest hypotonic stress is exerted to the cells by exposing them to distilled water (1 mOsm). This treatment leads to an extreme volume increase (Figure 76) and cell lysis (Figure 76 B, white arrow) upon exposure longer than 30 min.
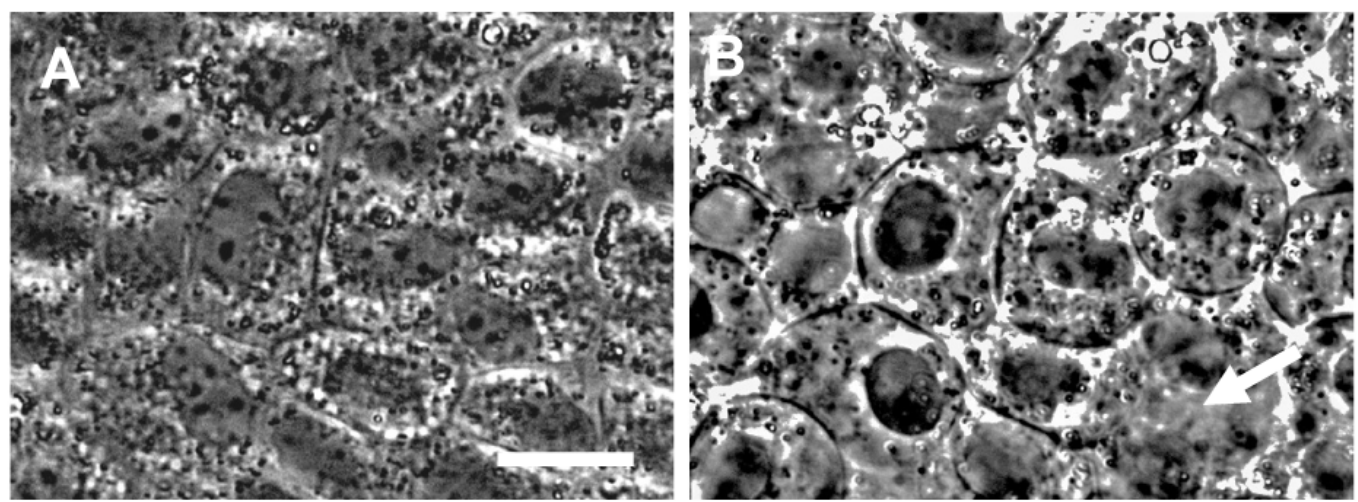

Figure 76: Phase contrast micrograph of MDCK II cells exposed to distilled water. A: untreated cells. B: treated cells. Whit arrow in right corner of the image indicates ruptured cell. Scale bar $20 \mu \mathrm{m}$.

This membrane area increase cannot be balanced due to tension regulating processes as observed in section 5.6.3. Inevitable this treatment leads to membrane lysis.
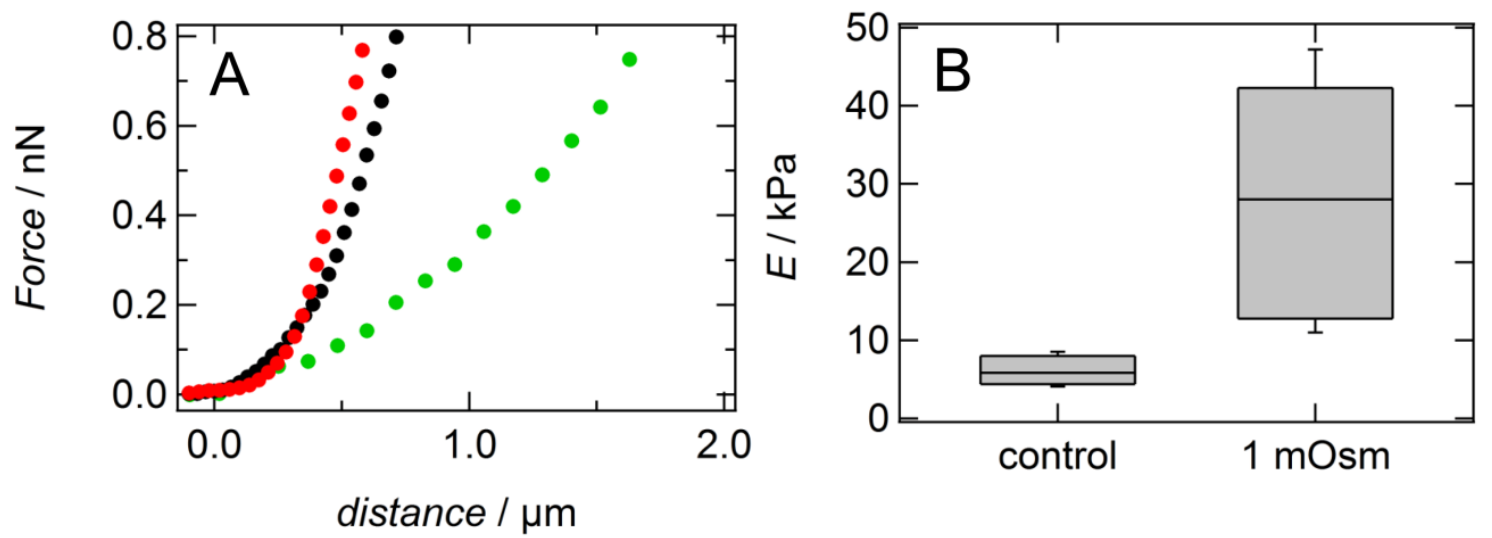

Figure 77: Mechanical investigation of MDCK II cells under different osmotic stress A: Force distance curves recorded on MDCK II cells after different treatments. (•) Green dots display mechanical response of untreated cells to indentation. (•) Black dots cells during hyperosmotic stress. (•) Red dots mark cells exposed to distilled water. B: Young's modulus of cells treated with very low osmolarity (distilled water) (n $=5$ cells).

Force distance curves recorded on this extremely swollen cells display a steeper slope compared to untreated cells (Figure 77). The hydrostatic pressure from the cytosol acting on the membrane results in a very high tension inside the bilayer, which cannot be buffered by reservoirs since they are exhausted. In order to place these experiments in a mechanical 
context the Hertzian contact model is used. The Young's modulus of MDCK II cells exposed to distilled water yield elevated values of $E=30 \mathrm{kPa} \pm 17 \mathrm{kPa}$. This is an increase in cell elasticity by a factor of 5 . The attempt to pull out membrane nanotubes of the plasma membrane of MDCK II cells exposed to distilled water fails. Not one tether could be extracted from the highly tensed membrane. Hence, no information about membrane tension is gained.

\subsubsection{Discussion}

Here, the impact of osmotic stress on the mechanical properties of the kidney epithelial cell line MDCK II is investigated with the AFM in a spatial-temporal fashion. Confluent monolayers were chosen to mimic tissue behavior in contrast to single isolated cells. We first examined the morphological changes due to hypoosmotic stress by analyzing AFM height images and observe an increase in cell height, while the diameter of the apical caps was preserved. The cell surface is increased at mild hypotonic treatment by a factor of 1.7 and at strong hypotonic stress by a factor of 2.7. This is in accordance with observations from Groulx and coworkers who found a 3.6-fold increase in surface area for mammalian cells 98\% hypoosmotic solution [21]. As the plasma membrane is largely inextensible and cannot bear large strains due to its liquid-crystalline nature limiting critical area dilatation to 3-4 \% [17], changes in surface area require a rapid response to maintain constant membrane tension in order avoid lysis. Cells increase their surface to avoid lysis due to volume increase by drawing additional membrane from preexisting reserves [20], which is visible in the AFM-topographical images. The cell surface is smoothened out since membrane protrusions like microvilli disappear during cell swelling at strong hypotonic treatment. Sukhorukov et al. could show by impedance measurements that hypoosmotically stressed cells obtain the necessary membrane area by using material from microvilli [57]. This membrane deformation has a detectable impact on the cellular mechanics. By performing indentation and tether pulling experiments we were able to show how cells respond to swelling or shrinking to maintain cell integrity and avoid lysis. A mild hypotonic stress leads to an increase in membrane tension because of the osmotic pressure acting against the membrane. The apparent area compressibility modulus also increases reflecting a 2-fold loss of excess membrane due to membrane deformation. This mild effect changes if the cells are subjected to stronger hypotonic stress. We observe a reorganization of the F-actin cytoskeleton, which counteracts the external force [6] by 
allowing the tension to relax. The phenotype resembles cells treated with cytochalasin D also producing the same mechanical response. Guilak and coworkers could also detect a decrease in the elastic module and F-actin distribution of chondrocytes by hypoosmotic challenge [130, 174, 175]. The remodeling of the cytoskeleton leads to a tension decrease and concomitantly to additional membrane area released from bound membrane reserves such as microvilli due to partial dissolution of the cortex encompassing also the microvilli. This scenario is confirmed by the fact that the apparent area compressibility modulus decreases considerably in response to strong hypotonic treatment. We interpret this sudden release of membrane material as a first line response to protect the cells from lysis due to swelling (Figure 78). The restoration of cell size under continuous hypotonic stress is known as regulatory volume decrease (RVD) [176]. During the experimental duration (30 min) we detect no such regulatory effect. As a consequence, the cell requires a fast response system to cope with mild to moderate changes in osmotic pressure. The question was now, whether the cells adapt to the situation by restoring some of the lost contacts between the membrane and the cytoskeleton and as a consequence regain the original membrane tension. Therefore, we extended the exposure time to hypotonic solutions to more than $6 \mathrm{~h}$ and found that the cell indeed largely restores its initial tension prior to exposure to hypertonic solutions. The accompanying increase in the apparent area compressibility modulus reflects how the cells accomplishes this task, namely by a reduction in surface area that is necessary to restore the tension. This picture is confirmed by fluorescence micrographs displaying enhanced endocytosis. The $\tilde{K}_{A}$-values measured after $6 \mathrm{~h}$ of exposure to hypotonic solutions is virtually identical than that measured for neat lipid bilayer lacking membrane reserves or local region with strong connection to the underlying cytoskeleton. A stronger adhesion to the cortex can be clearly inferred from tether pulling experiments yielding increasing tether forces with time and fluorescence microscopy showing an increasing amount of ezrin and actin at the apical side. This means that the cells have sacrificed the entire excess surface stored in protrusions and invaginations to restore the original tension. Probably this is at the upper limit for osmotic stress cell can bear without damage. Interestingly, the basal side of the polar epithelia seems to be less prone to remodeling of the actin cytoskeleton (lack of stress fibers), which suggests that the process of adaption has not been finished yet. 


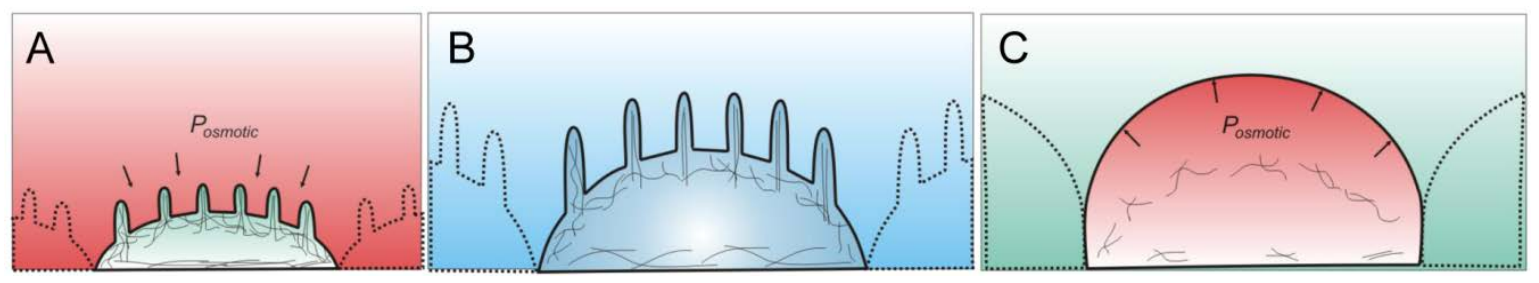

Figure 78: Schematic illustration of osmotic challenged MDCK II cells. A: Cell shrinkage due to hyperosmotic stress acting outwards on the plasma membrane. B: Cells under isotonic conditions exposing membrane reserves and underlying cortex. C: Cell swelling due to osmotic pressure acting against the internal plasma membrane (hypoosmotic conditions).

In contrast to hypotonic stress, hyperosmolar stress merely leads to collapsing of the membrane on the actin cortex (Figure 78). As a consequence, the cell morphology changes tremendously, which impacts the appearance of force indentation curves severely. The cells appear much stiffer [177] [178], which is mainly a product of the switch in topography rather than a sudden increase in stiffness. By accounting for the changed geometry we arrive at reasonable values for the area compressibility and tension. Tension values derived from tether experiments are trustworthier reporting that tension slightly increases. Tension derived from indentation also comprises elastic contributions from bending which are measured in this study. This can be attributed to the collapse of the apical plasma membrane on the underlying cortex and a remaining osmotic pressure pointing inwards and as a consequence increase the tether force.

Exposing MDCK II cells to very low osmolarity (1 mOsm, distilled water) display a tremendous volume change [21] [179]. The high hydrostatic pressure of the cytosol acting on the plasma membrane generates tension inside the bilayer, stretching out all membrane reserves [20]. This limits the use of the liquid droplet approach, because the constant intrinsic membrane tension is over exceeded by osmotic pressure. Tether pulling experiments fail, since no tether could be extracted from the high tensed membrane. In order to place these experiments in a mechanical context the Hertzian approach is used, yielding an increase in measured cell elasticity by a factor of five. 


\subsection{Mechanics of Spreading Cells ${ }^{9}$}

Cellular adhesion and motility are crucial processes in biological systems such as morphogenesis, wound healing and tissue homeostasis. For instance, lymphocytes migrating through an intact cellular layer rely on the ability to adhere and move through tissue [22]. Sahai and Marshall report that tumor melanoma cells produce dynamic blebs, which allow migration through a 3D matrix [180]. Experiences from these studies clearly show that deformation and supply of excess membrane are key factors for the above mentioned processes. While the morphological changes taking place during spreading of cells to a substrate or matrix are well known and characterized for different cell lines, comprehensive studies of the changes in cell mechanics are scarce, although it is known that membrane tension and cell motility are strongly interwoven. Generally, the spreading process can be divided into three phases [173] [172]. The first phase is characterized by the formation of initial bonds followed by the second phase comprising the initial cell spreading, which is driven by actin polymerization that forces the cell surface area to increase by drawing from a membrane reservoir of folded regions and blebs. Blebs are not only a characteristic for apoptosis [181], cell division [182] but also for cell spreading [183]. Cells in suspension frequently display blebs, which originate from a drop in tension such as the one experienced upon detachment of an adhered cell. These membrane protrusions appear only transiently during the spreading progress and vanish at a later stage . After the depletion of this membrane reservoir, the cells enter the third spreading phase, whereby additional plasma membrane is recruited from internally stored membranes buffer [184]. The various stages are accompanied by substantial changes in cellular mechanics to accommodate challenges posed by spreading and migration. Here, we study the mechanics of spreading, as a paradigm for the close connection between cell shape and membrane mechanics using atomic force microscopy. Spreading of a cell is a F-actin dependent process as cells spread very slowly after disruption of the actin cytoskeleton [185], while the plasma membrane has mechanically to deform from a spherical to a nearly discoidal form, promoting the idea that membrane mechanics, i.e. the in-plane tension, limits cell spreading. Stretching an epithelial cell causes the cell to withdraw lateral protrusions while suppression of spreading activity is reversed upon release of tension [23]. Raucher and

\footnotetext{
${ }^{9}$ Main part of this chapter will be published in Pietuch, A.; Janshoff, A. Mechanics of spreading cells probed by atomic force microscopy. Open Biol. 2013 Jul 17; 3(7):130084.
} 
Sheetz reported that chemically induced reduction of membrane tension leads to faster cell spreading [9]. This suggests that the plasma membrane acts as a regulator of cell spreading through tension homeostasis limiting the number of protrusion due to exhaustion of membrane reservoirs. Indentation experiments combined with site-specific pulling of membrane tethers, both carried out with the atomic force microscope (AFM) allowed us to assess simultaneously local changes in membrane tension and excess area during the spreading of epithelial cells. During adhesion and spreading, the shape of a suspended cell changes from a sphere to a capped sphere. Cell shape changes were taken into account by adjusting the geometrical constraints of a liquid droplet model describing the cell as liquid filled body with an isotropic in-plane tension [24] [25]. These experiments are supported by fluorescence microscopy of the plasma membrane as well as visualization of F-actin of adhering and spreading cells.

\subsubsection{Changes in Cellular Mechanics during Cell Spreading}

The mechanical behavior of a spreading MDCK II cell is monitored as a function of time using force measurements to characterize this process in terms of varying membrane tension and concomitant surface area regulation. Indentation of a cell with an AFM tip provides force vs. tip-sample-separation curves (FC).

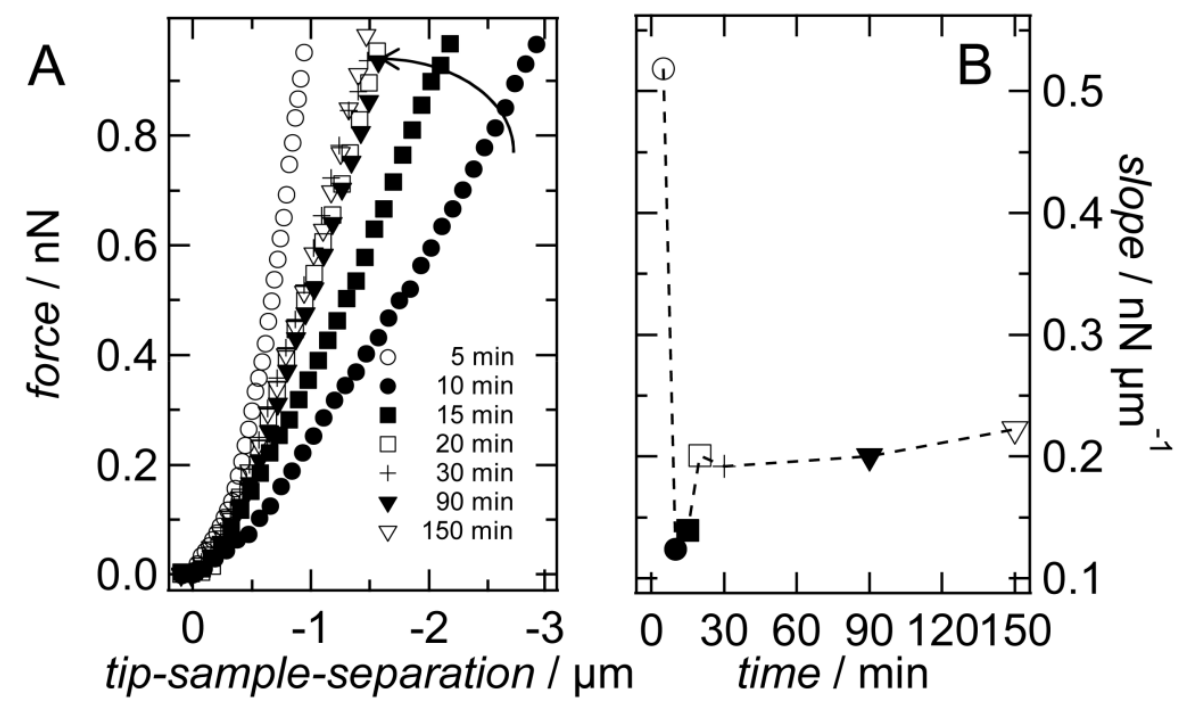

Figure 79: Mechanical properties of MDCK II cell during adhesion and spreading. A: Force curves taken at different time point during the spreading process. Force curve taken 5 min after cell seeding (open circle) shows the steepest slope followed by a sudden softening (10 min, black circle). Within $30 \mathrm{~min}$, force curves snap back to higher stiffness. B:Slope of FCs plotted against time. 
Figure 79 A and B shows a shift in the slope of FCs taken on a single cell during adhesion and spreading, which is generally observed in all experiments. The first FC is taken 5 min after cell seeding, displaying an indentation depth of $1 \mu \mathrm{m}$ at the given set point of $1 \mathrm{nN}$. 5 min later a tremendous decrease in the slope of the FC is found mirrored in an increased indentation depth of $3 \mu \mathrm{m}$. Following the spreading progress over the next 3 hours, reveal a reversal of the slope towards its initial value. Even without an appropriate mechanical model of the cell a change in the slope is indicative of a substantial change in the cell's stiffness. A higher indentation depth at constant force could only be reached if the cells soften, more precisely; if the cells obtain access to a membrane reservoir, which allows to compensate for the stress exerted by the indenter. This initial softening is followed by a gradual increase in cell stiffness towards a constant value (> $20 \mathrm{~min})$.

\subsubsection{Morphological Changes during Adhesion and Spreading}

Optical phase contrast images are recorded concomitantly to mechanical investigation to follow cell shape changes during adhesion and spreading (Figure $80 \mathrm{~A}-\mathrm{D}$ ).
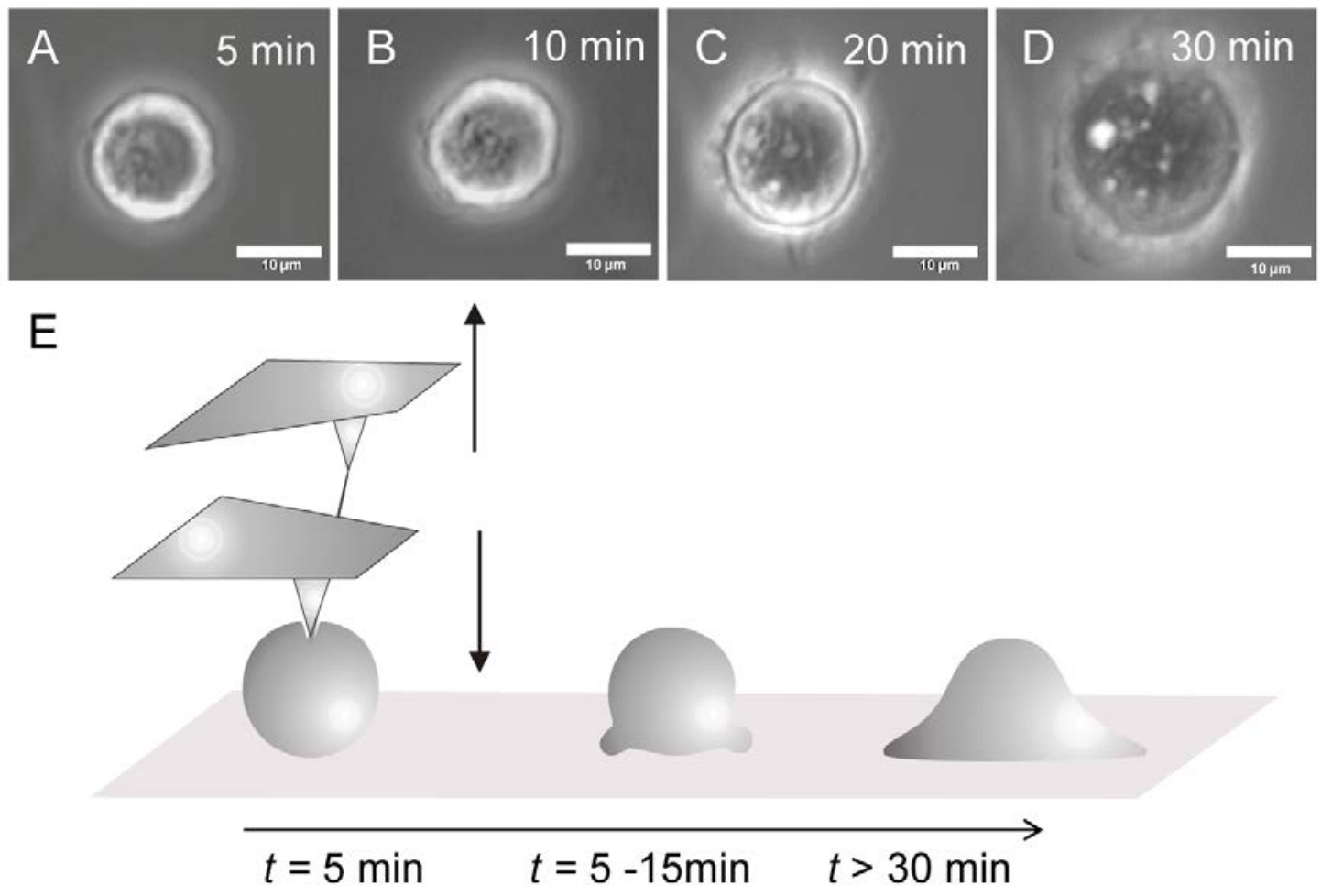

Figure 80: A-D Light microscopy images taken at different time points documenting the spreading behavior of MDCK II cells. E: The adhering and spreading cell is locally indented with an AFM tip resulting in force curves shown exemplary in Figure 79. While retracting the cantilever a membrane nanotube (tether) is frequently pulled out of the plasma membrane bearing additional mechanical information. 
Cells in suspension exhibit a spherical shape, frequently displaying blebs (Figure 81).
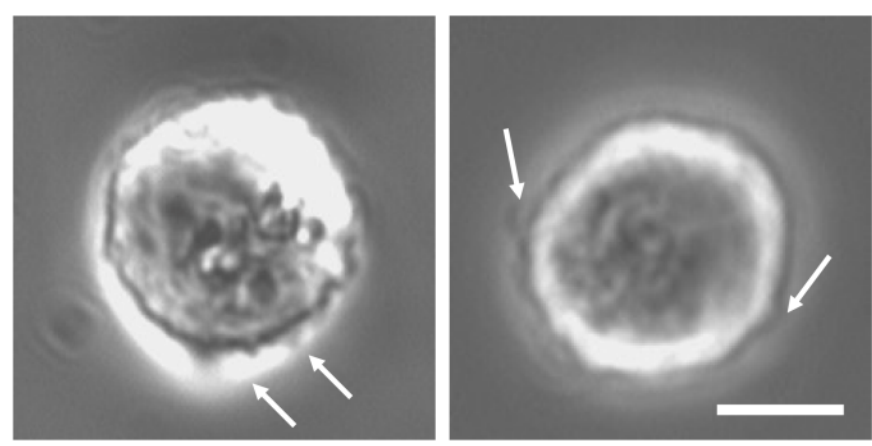

Figure 81: Phase contrast micrographs of MDCK II cell in suspension. Cells display membrane protrusions, indicated by white arrows. Scale bar is $10 \mu \mathrm{m}$.

First significant cell shape changes can be observed after $10 \mathrm{~min}$, where the first spherical membrane protrusions are visible forming the initial substrate adhesion area (Figure $80 \mathrm{~B}$ ). This initial adhesion phase is followed by a substantial increase in adhesion area (Figure 80 20-30 min), which is referred to as the initial spreading phase. The dynamics of cell spreading has been studied for many cell lines drawing a comprehensive picture of this process. Bereitener-Hahn and coworkers showed that the initial phase is characterized by the attachment of the cell to the substrate forming a broad and smooth contact area (within the first $10 \mathrm{~min}$ ) followed by a broadening of the adhesion zone through cytoplasmatic flow. The final cell spreading is characterized by the extension of lamellae [186]. Observing the spreading area over time, Norman et al. found that the contact area stays constant after 40-60 min [183]. A similar time dependent spreading behavior for MDCK II cells is observed leading to the chronological sequence illustrated in Figure 80 E. The next step in modeling the mechanical response is to devise an appropriate model of the cell that captures the essential mechanical features encompassing tension, area compressibility, and surface area. The cell shape needs to be considered carefully as it bears important input parameters for any mechanical model that depends on the cell geometry (vide infra).

\subsubsection{Mechanical Models - Change from Sphere to Caped Sphere}

Generally, we assume that MDCK II cells behave as a liquid droplet with an isotropic inplane tension. For the initial spreading phase a model described by Rosenbluth et al. is chosen, where the cell is treated as an unperturbed sphere prior to indentation (Figure 82) [24]. While the initial model assumes a pyramidal indenter, we modified the theory to describe indentation with a conical tip (section 3). 

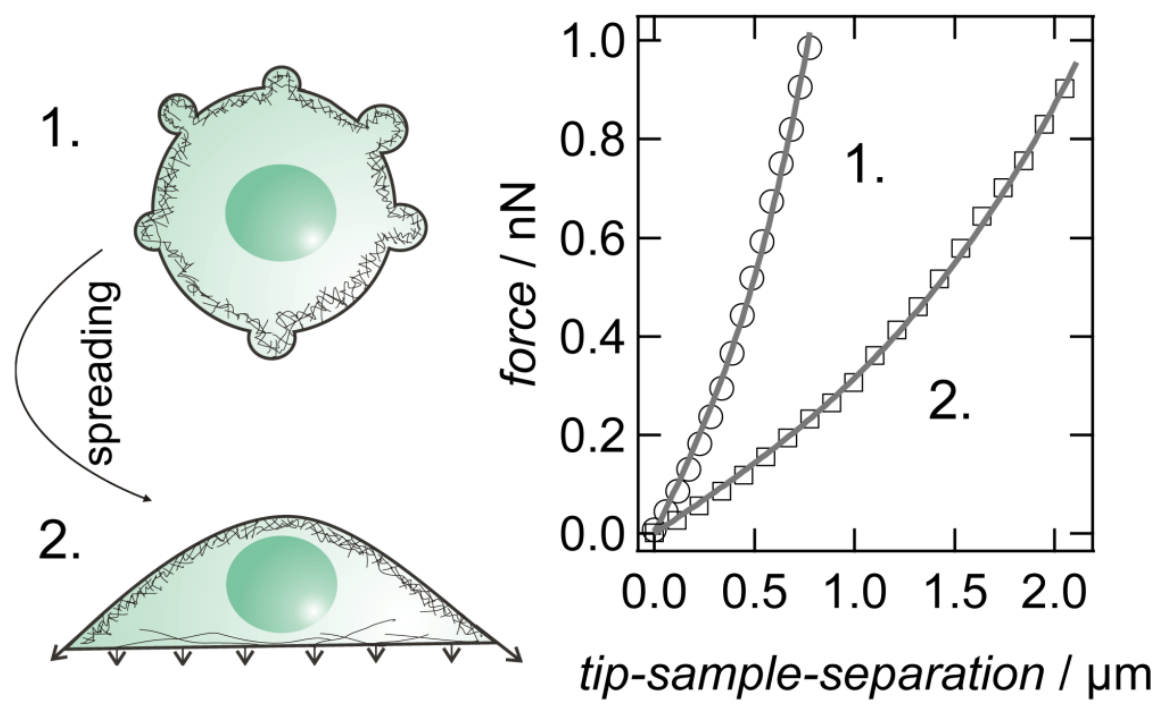

Figure 82: Mechanical model is changed from a sphere to a caped sphere. (Right) Force curves taken on a spherical cell (dots) and spreading cell (squares) with the corresponding fit using membrane theory (section 3).

When the cell starts to adhere and spread the shape changes from a sphere to a capped sphere. 15 min after adhesion the model is switched to one described by Discher and coworkers, who compute the exact cell shape starting from a spherical cap (Figure 82) [25]. Both models assume a constant isotropic tension $T_{0}$ extended by an area dilatation term that accounts for in-plane stretching during indentation. For detailed description of the used models the reader is referred to section 3.

Apart from morphological observation via microscopy (Figure 82), the time point for switching the model is depict from fitting both mechanical approaches to experimental data as shown in Figure 83. Membrane tension $T_{0}$ obtained from both mechanical models shows nearly the same trend (Figure $83 \mathrm{~B}$ ). For the capped sphere approach $T_{0}$-values are elevated by a factor of 3 compared to spherical modulation of the cells shape. However, the area compressibility modulus displays an interesting course (Figure $83 \mathrm{~A}$ ). 

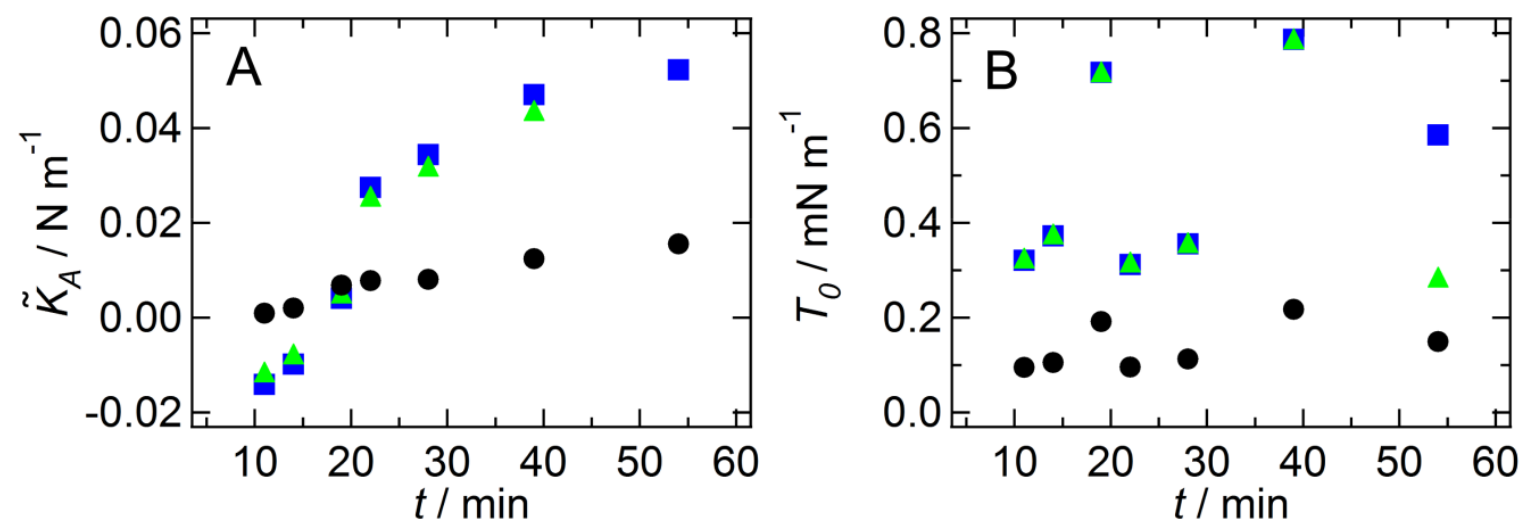

Figure 83: Mechanical parameter obtained from fitting the liquid droplet model for spherical $(\bullet)$ or adherent cell (( $(\square)$ for $\phi=75^{\circ},(\Delta)$ for $\left.\phi=21^{\circ}\right)$ to experimental data obtained from adhesion and spreading process of a MDCK II cell.

While $\tilde{K}_{A}$ - values for spherical shape increases constantly over time from $0.001 \mathrm{~N} \mathrm{~m}^{-1}$ (10 min) up to $0.02 \mathrm{~N} \mathrm{~m}^{-1}$, capped sphere modulation leads to negative $\tilde{K}_{A}$ - values for the beginning of adhesion and spreading $\left(-0.01 \mathrm{~N} \mathrm{~m}^{-1}\right)$. This clearly demonstrates that modulation of a spherical cape is not suitable to describe the beginning of the spreading process.

\subsubsection{Mechanical Parameter during Adhesion and Spreading}

The mechanical parameters obtained from shape-dependent FC fitting display a dramatic change in the initial phase of adhesion and spreading (Figure 84). The apparent area compressibility modulus $\tilde{K}_{A}$, reflecting the change in membrane area, drops from $0.19 \mathrm{~N} / \mathrm{m}$ $\pm 0.01 \mathrm{~N} / \mathrm{m}(5 \mathrm{~min})$ to a value of $0.006 \mathrm{~N} / \mathrm{m} \pm 0.006 \mathrm{~N} / \mathrm{m}(15 \mathrm{~min})$, indicative of a substantial increase in available membrane area. This is followed by an increase in $\tilde{K}_{A}$ and leveling off to an almost constant module of $0.05 \mathrm{~N} / \mathrm{m} \pm 0.02 \mathrm{~N} / \mathrm{m}$. At the same time a decrease in the membrane tension $T_{0}$ is detected from an initial value of $0.29 \mathrm{mN} / \mathrm{m} \pm 0.06$ $\mathrm{mN} / \mathrm{m}$ to $0.13 \mathrm{mN} / \mathrm{m} \pm 0.04 \mathrm{mN} / \mathrm{m}$ (Fig. $2 \mathrm{~F}$ ). After this relaxation (15 min) the tension restores to an almost constant value of $0.6 \mathrm{mN} / \mathrm{m} \pm 0.05 \mathrm{mN} / \mathrm{m}$.

Indentation experiments are supported by tether pulling experiments taken on the same spot, representing an independent mechanical approach for probing membrane properties. Membrane tension contains contributions from membrane-cytoskeleton-adhesion, the contraction of the actomyosin cortex as well as tension in the bilayer itself [124]. The 
tension $T_{t}$ determined from tether pulling also shows a decrease within the first $30 \mathrm{~min}$ of adhesion and spreading $(0.54 \mathrm{mN} / \mathrm{m} \pm 0.03 \mathrm{mN} / \mathrm{m}$ to $0.16 \mathrm{mN} / \mathrm{m} \pm 0.14 \mathrm{mN} / \mathrm{m})$. Eventually, $T_{t}$ increases to a final value of $0.38 \mathrm{mN} / \mathrm{m} \pm 0.11 \mathrm{mN} / \mathrm{m}$.

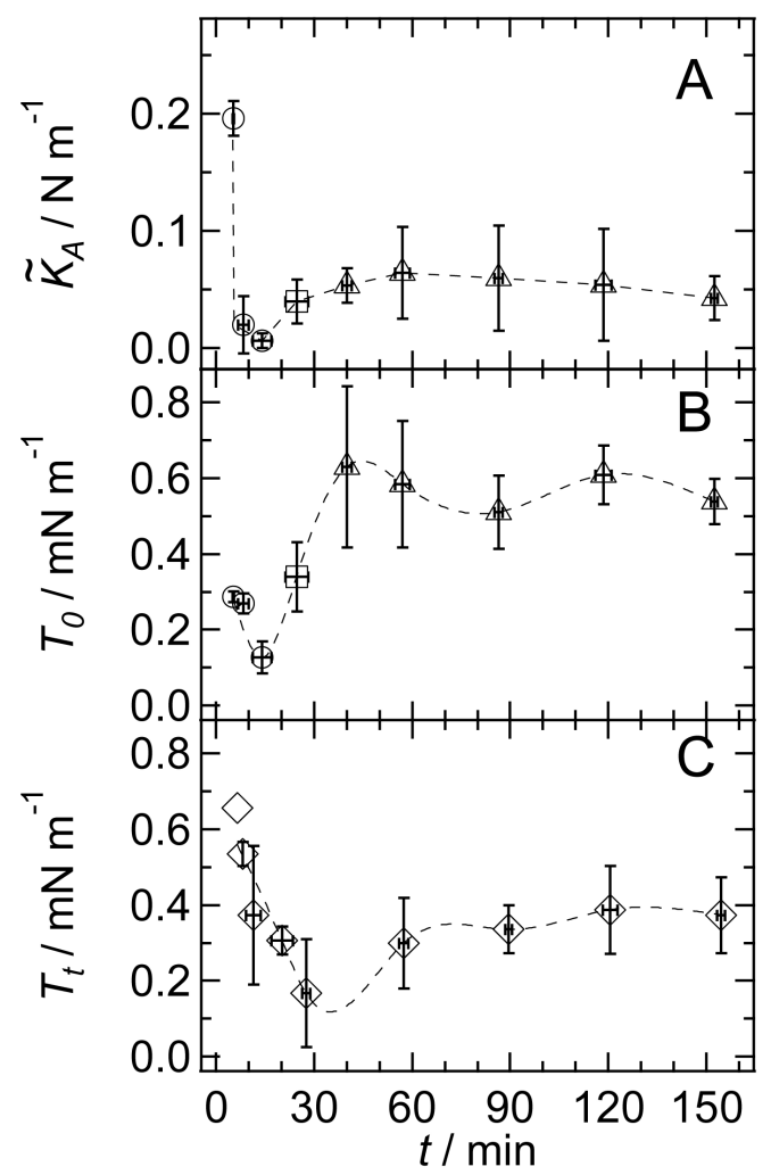

Figure 84: Mechanical parameter of adhering and spreading MDCK II cells $(n=4)$. Apparent area compressibility modulus and membrane tension $\left(T_{0}\right.$ and $\left.T_{T}\right)$ are monitored during spreading progress. Circles correspond to model for spherical cells, while squares represent mechanical parameters obtained from model of an adhering cells with $\phi_{0}=75^{\circ}$. Triangles represent modeling of a spread cell with $\phi_{0}=21^{\circ}$. C: Membrane Tension $T_{t}$ calculated from tether pulling experiments.

Both mechanical measurements, indentation as well as tether pulling, reveal the same trend in membrane tension, first a decrease followed by an increase and eventually a constant value. This supports the course of membrane tension $T_{0}$ where the mechanical model is switched after 15 min for modulating the cellular shape from a sphere to a capped sphere.

Further spreading and polarization of a single cell (24h) does not change measured membrane tension (Figure 85). 


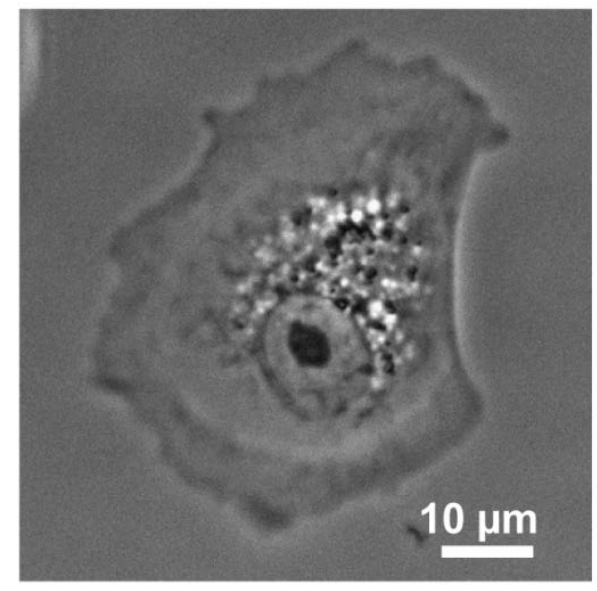

Figure 85: Phase contrast micrograph of full spread MDCK II cell 24h after cell seeding.

Here, we obtain from indentation experiments a $T_{0}$ value of $0.6 \mathrm{mN} / \mathrm{m}$ and from tether pulling a $T_{t}$ of $0.3 \mathrm{mN} / \mathrm{m}$. Notably, the value obtained from either pulling or indentation are virtually identical confirming that the mechanical models shown in section 3. largely capture the elastic properties of adhering MDCK II cells.

\subsubsection{Spherical Cell- Before Adhesion and Spreading}

Cells in suspension frequently display membrane protrusions that are lined with actin filaments (Figure 81 and Figure 86). Size and growth dynamics depend on cortical tension [155]. A sudden drop in membrane tension, as it occurs upon detachment of an adherent cell, nucleates formation of protrusions probably due to the contractile nature of the actomyosin cortex that restores tension in the plasma membrane through increase in the internal pressure. A lack in excess area can be inferred from an increase in the apparent area compressibility modulus. The overall tension of the plasma membrane is a result of contributions from surface tension of the lipid bilayer, adhesion, molecular connection of the plasma membrane and the underlying actin-cytoskeleton [10], and an active contribution from the contractile actomyosin cortex [187] [188]. When membrane tension is chemically decreased, faster cell spreading is observed, supporting the idea that cell membrane mechanics play an important role in controlling membrane protrusions and therefore spreading [9]. 


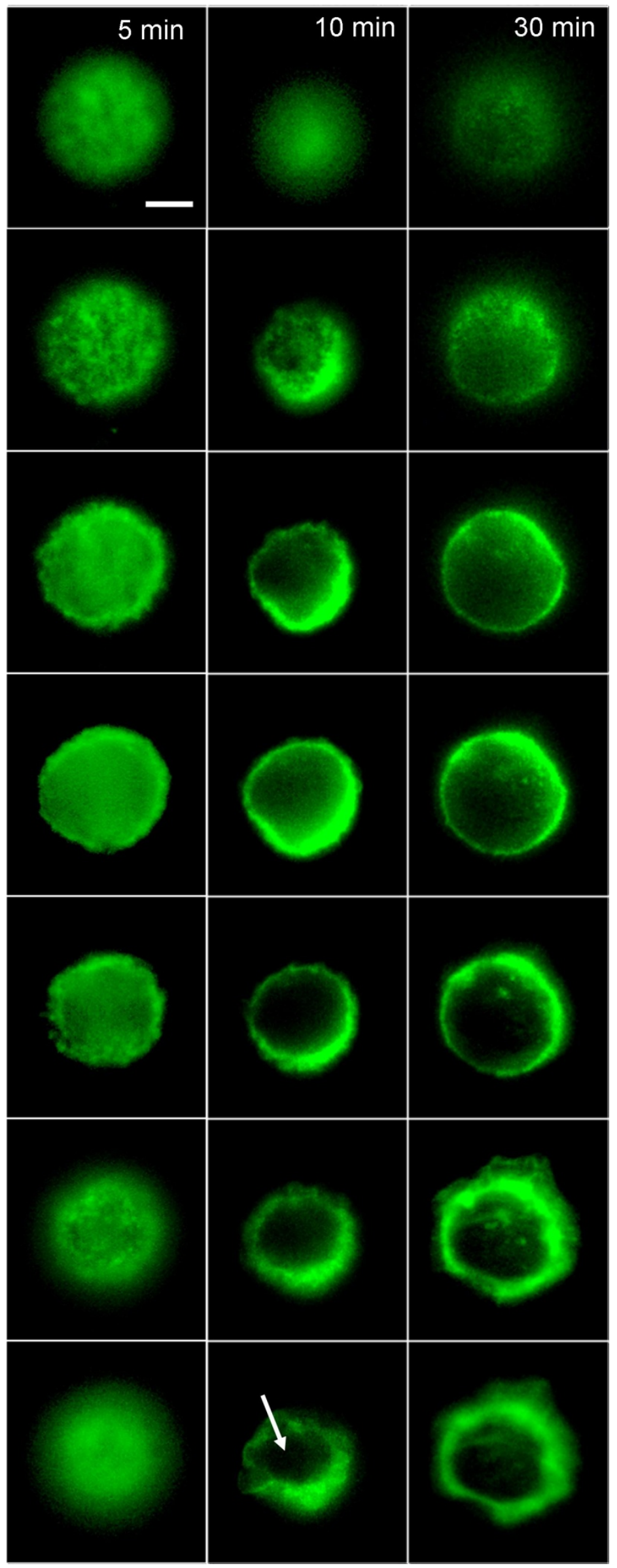

Figure 86: Fluorescence micrographs of F-actin stained spherical MDCK II cell 5 min after cell seeding (left column), an adhering cell $15 \mathrm{~min}$ after cell seeding (middle column) and spread one 30 min after cell seeding (right column). One column displays slices through the cell starting from the upper part. It should be pointed out, that $10 \mathrm{~min}$ after cell seeding, F-actin distribution changes. The prior uniform actin layer (cortex), as observed for a cell after 5 min, disappears in the basal part and membrane blebs start to form. 
Although the membrane of a spherical cell displays a rougher, more corrugated surface than a cell after adhesion and spreading (Figure 86, Figure 87), the measured apparent area compressibility module $\tilde{K}_{A} 5$ min after cell seeding shows a value of $0.19 \mathrm{~N} / \mathrm{m}$, essentially identical with a neat lipid bilayer in the absence of a buffering membrane reservoir. Equation 19 enables to calculate the excess membrane area. Area compressibility modules of pure lipid bilayers and cell membranes are in the range of $0.1-1 \mathrm{~N} \mathrm{~m}^{-1}$ depending on their composition [71] [131]. Cells with such high $K_{A}$ values obviously possess no excess membrane area $A_{e x}$. This confirms that a cell in its spherical stage has no access to excess membrane. This is also supported by the large forces needed to pull tethers from cells in the spherical state.

High tether forces reflect a stronger membrane binding to the underlying cortex. In order to provide additional evidence for our model we labeled the F-actin in these spherical cells (Figure 86 left column) displaying a uniform cortex. The blebs or protrusions occurring in the initial stage after adhering are therefore lined with actin filaments. This continuous and rigid actin cortex provides resistance to mechanical forces and therefore protection against damage. Hence, non-adhering cells or cells just before adhesion show a high area compressibility modulus and membrane tension (Figure 84).

\subsubsection{First and Second Phase of Cell Spreading}

Within 10 min after seeding cells adhere to the substrate by the formation of an initial adhesion zone [189]. Exactly at this time a relaxation in tension and a sudden increase in the available membrane area are detected, reflected by a lower area compressibility module $\tilde{K}_{A}$. This corresponds to an increase of excess membrane area by a factor of 12 (Equation 19). Additionally, a disruption of the F-actin layer in the basal area of an adhering cell is observed (Figure 86, arrow in middle column) accompanied by a reduction in membrane tension (Figure 84), confirming a weakening of F-actin that supports the membrane. This temporary release in tension (Figure 84) enables the cell to spread on the substrate by forming the first extensions, also visible in light microcopy (Figure 80) and fluorescence micrographs (Figure 87) as large spherical extensions (blebs) in the lower part of the cell. 
Figure 87: Fluorescence micrographs of a spherical MDCK II cell (membrane staining) 5 min after cell seeding (left column), an adhering cell 15 min after cell seeding (middle column) and a spread cell 30 min after seeding (right column). Left column shows slices through a spherical cell, beginning at the top. Middle column shows corresponding images through an adhering cell, and right column through a spreading cell. Scale bar 20 $\mu \mathrm{m}$.

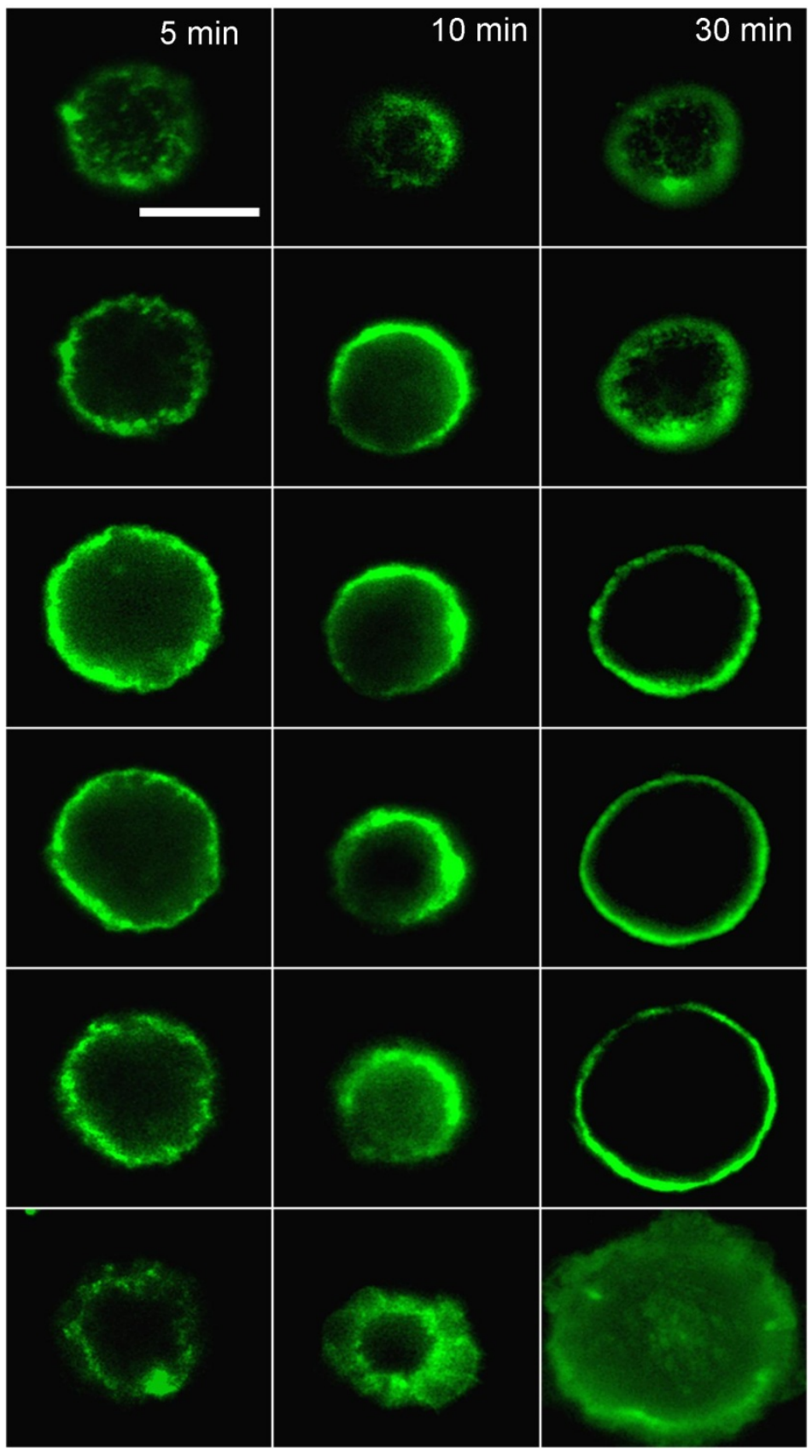

The breakdown of the F-actin cortex is followed by a bulk flow of cytoplasm causing a bleb to protrude, which allows spreading on the glass surface [186]. The nucleation of a membrane bleb is provoked by a local weakening of the cortical complex and promoted by the internal pressure of the cell generated by the contractile actomyosin cortex [155] [156, 190]. The drop in tension causes the change from a static and rigid to a more dynamic actin cortex that allows spreading by drawing additional membrane from reservoirs stored in protrusions, invaginations or internal membrane structures [191] [192]. Ren and coworkers observed a decrease in Rho activity when suspended cells adhere to a matrix [193]. Rho 
regulates the formation of actin stress fibers [194] and integrin signaling [195]. Upon inhibition of Rho, the cortical F-actin network is disrupted, thereby allowing integrins to be clustered, which are otherwise dispersed over the cell surface in a suspended cell [196] [197], which allows the cell to first adhere and finally to spread. Therefore, the decrease in tension and increase in excess membrane area is attribute to a breakdown of the cortical Factin in the basal part of the cell causing membrane to protrude at these defects forming the initial adhesion zone.

\subsubsection{Inhibition of Adhesion Abolishes Tension Drop}

The adhesion of a spherical cell to the substrate leads to a release of tension and excess membrane area. If this hypothesis is correct, the inhibition of adhesion should prevent a drop in the mechanical parameters. Hence, the behavior of a MDCK II cell in suspension is investigated by coating the substrate with BSA and therefore abolishing cellular adhesion to a large extent [198].
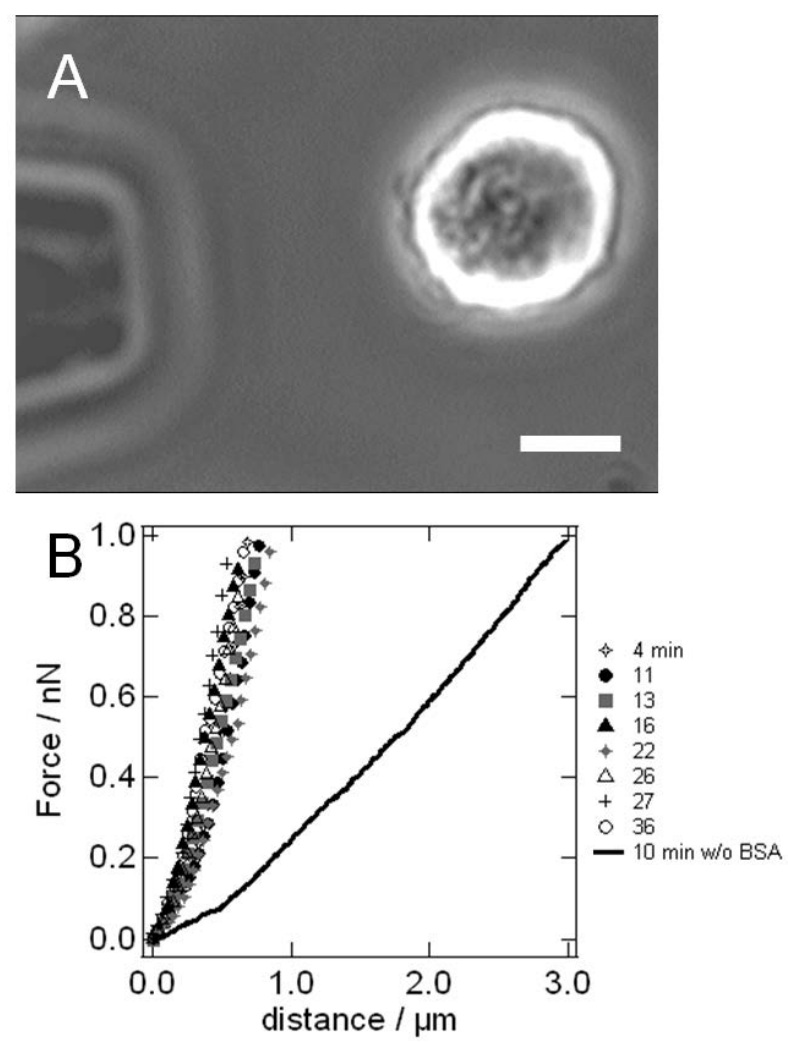

Figure 88: Phase contrast image of a spherical, non-spread MDCK II cell on BSA coated surface. In left corner the cantilever is visible. Scale bar $10 \mu \mathrm{m}$. B: Force distance curves taken over time on a non-spreading MDCK II cell. Slope of FC does not change over time. For comparison, a FC of adhering and spreading cell is plotted (in black). 
While challenging the cells mechanically, no adhesion to the substrate is observable. In addition, the barely adhering cells display many protrusions emphasizing that they are arrested in the state of initial adhesion (Figure 81). Figure 88 shows FCs taken continuously on a spherical cell. There is no change in the slope of recorded FCs over a time period of more than $30 \mathrm{~min}$ and the indentation depth is not exceeding $1 \mu \mathrm{m}$.

Comparing these data with the slope of a FC taken 10 min after cell seeding on a untreated substrate it becomes evident that no adhesion and spreading of spherical cells occurs on a BSA coated substrate (black line Figure 88 B). This supports the finding that the adhesion process is accompanied by a characteristic change in tension and surface area, marked by an initial drop in tension followed by slow restoration.

\subsubsection{Third Phase of Cell Spreading}

After adhesion and subsequent formation of membrane protrusions to enable spreading, the substrate adhesion zone increases concomitantly. At the same time, membrane protrusions, invaginations and blebs are smoothed out. Figure 87 shows membrane staining of MDCK II cells during the adhesion and spreading process. Comparing the membrane roughness of a just adhered cell with a fully spread one reveals a reduction in membrane protrusions after spreading (left column and right column). This is in good agreement with a study of Norman et al. who describe that blebs disappear 30-40 min after seeding of cells [183]. While increasing the contact zone with the substrate by smoothing out membrane reservoirs, the apparent area compressibility modulus $\tilde{K}_{A}$ increases accordingly to an almost constant modulus of $0.05 \mathrm{~N} \mathrm{~m}^{-1}$, which corresponds to a membrane folding factor of only 2 (Figure $84 \mathrm{~A}$ ). Simultaneously, membrane tension measured by indentation as well as tether pulling shows a recovery to $0.37 \mathrm{mN} \mathrm{m}^{-1}$ (Figure $84 \mathrm{~B}, \mathrm{C}$ ). At this time first stress fibers become visible indicative of a fully established adhesion to the substrate (Figure 86 right column).

\subsubsection{Conclusion}

Combining the results from mechanical probing and fluorescence microscopy a mechanical model for the adhesion and spreading of a suspended MDCK II cell is proposed. 


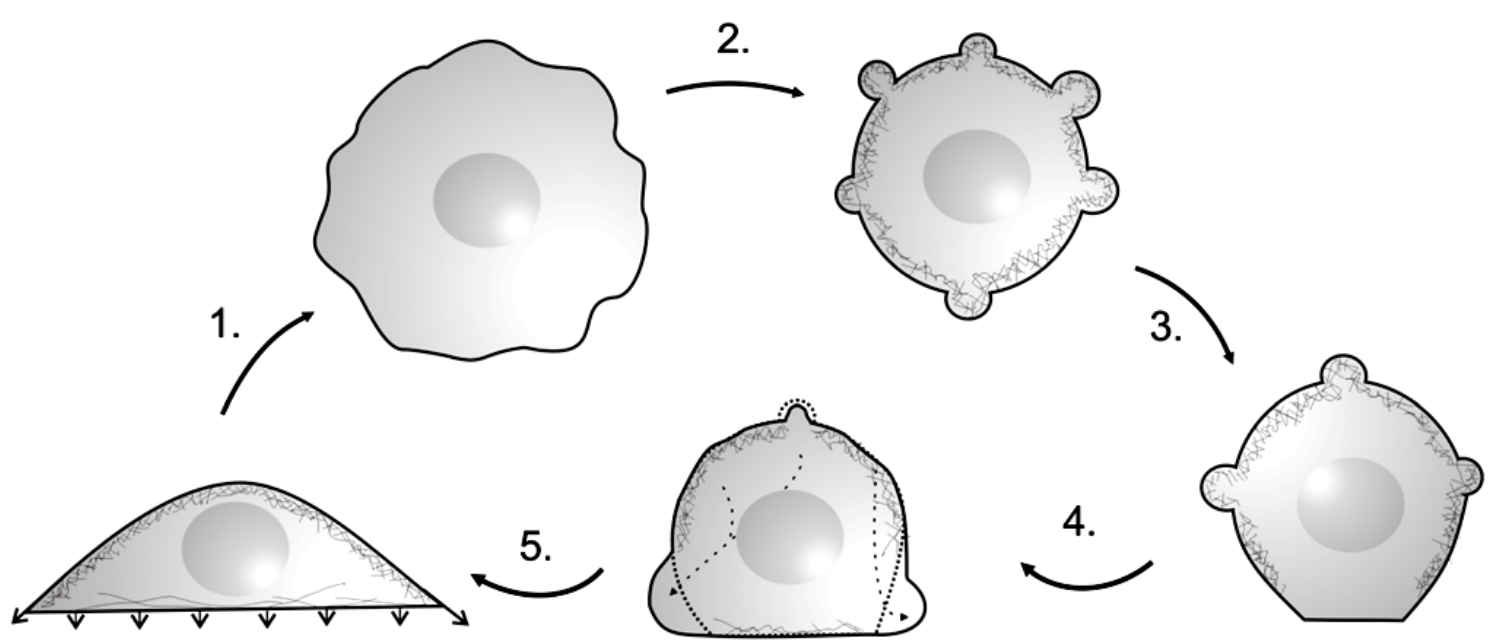

Figure 89: Illustration of the key events in cellular adhesion and spreading deduced from mechanical experiments as a function of time. (1.) Detachment of an adherent cell due to trypsinization. Freshly detached cells restore tension by creating actin-coated protrusions that consume excess membrane to maintain tension (2.). Due to adhesion, the actin cortex partially opens close to the substrate (3.) allowing a bulk flow of cytoplasma to cause blebs as an onset of spreading (4.). In the last step cells start to flatten and the cell's surface becomes smoother while stress fibers form (5.).

If a cell is detached from the substrate, membrane tension decreases causing the formation of membrane protrusions (Figure 89 1.). Tension is subsequently restored (Figure 84) by enclosing excess membrane into these actin-lined protrusions, reflected in a high area compressibility modulus close to that found for inextensible lipid bilayers (Figure 84 and Figure 89 2.).

Adhesion to the substrate leads to a partial rupturing of the cortical actin and drop of tension along with a dramatic increase in the excess membrane area (factor 12) (Figure 89 3.). This is essential for the cell to spread by creating membrane protrusions originating from the basal part of the cell (Figure 89 4.). In the course of spreading, membrane tension increases again by smoothing out existing membrane reservoirs a result of tension homeostasis controlled by surface area regulation (Figure 89 5.). After occurrence of first stress fibers tension and surface area maintain a constant level indicating full adhesion of the cell to the substrate.

In conclusion, time resolved mechanical probing of cellular elasticity of the spreading process reveals that cell spreading requires a transient release in tension to permit formation of membrane protrusions. It is conceivable that a similar mechanism is responsible for to accommodate the dynamic remodeling of the cytoskeleton during cell migration. 


\section{Final Discussion and Concluding Remarks}

The conventional method to determine membrane tension or the area compressibility modulus of the cellular plasma membrane is the micropipette aspiration either in combination or separately laser tweezer technique to pull out membrane nanotubes. In the present work, a different mechanical approach is established to quantify plasma membrane mechanics of adherent cells by using the atomic force microscope. It was assumed that MDCK II cells behave as a liquid droplet with an isotropic in-plane tension. The typical non-linearity observed in force indentation experiments performed on epithelial cells suggests that membrane theory with an additional pre-stress term is more suitable to describe cellular elasticity of fluid-like cells than assuming a homogeneous elastic body characterized by a single Young's modulus. The theory of membrane mechanics from Sen et al. was extended to adherent and confluent cells mimicking the cellular behavior in an intact tissue. Indentation experiments were accompanied and verified with pulling of membrane tethers as second mechanical approach. It was found that both techniques provide essentially identical values for the lateral tension of untreated cells $\left(\sim 0.3 \mathrm{mN} \mathrm{m}^{-1}\right)$. Similar values were obtained from micropipette suction experiments of neutrophil membranes [106, 135]. This is a clear hint that the tension $T_{0}$ measured in indentation experiments is due to in-plane tension of the membrane shell originating from the attachment of the plasma membrane to the cytoskeleton and adherens junctions and is not an elastic property of the actin cortex (terminal web). The area compressibility modulus $\tilde{K}_{A}$ of untreated MDCK II cells displays a bimodal distribution centering on a low (peak I, $0.02 \mathrm{~N} \mathrm{~m}^{-1}$ ) and a high (peak II, $0.1 \mathrm{~N} \mathrm{~m}^{-1}$ ) $\tilde{K}_{A}$ value, respectively. Disrupting the F-actin cytoskeleton with cytochalasin D led to a structural rearrangement of the apical F-actin and to a change in the distribution of the co-localized protein ezrin. The depolymerization of Factin decreased the membrane tension by a factor of 3. The alteration in cortex integrity showed also a change of available membrane area, manifested in a slight lowering of peak II, while peak I representing lower $\tilde{K}_{A}$ values remains unaffected (Figure 49). We found some evidence that this distribution reflects two distinct topographical regions on the apical cell membrane by fluorescence microscopy. One region is associated with an extended membrane reservoir, giving rise to a low $\tilde{K}_{A}$ and a second population, in which the membrane constitutes a thin stretched shell that shows an area compressibility modulus 
resembling those of artificial lipid bilayers or membranes from red blood cells [131, 136, 137]. Probing the elasticity of isolated planar apical cell membrane fragments, we found a mean tension $T_{0}$ of $0.16 \pm 0.07 \mathrm{mN} \mathrm{m}^{-1}$ and apparent area compressibility modulus of 0.009 $\pm 0.001 \mathrm{~N} \mathrm{~m}^{-1}$ representative for a plasma membrane lacking cytoskeleton filaments but associated to a lipid reservoir providing excess area upon stretching the planar membrane. The value corresponds well to the apparent values found for vital MDCK II cells. This clearly demonstrates that the elastic response of the plasma membrane due to tension regulated by the surface area and not the properties of the underlying cortex are probed. If the low values of the apparent area compressibility module $\tilde{K}_{A}\left(0.02 \mathrm{~N} \mathrm{~m}^{-1}\right.$, found for untreated cells) were indeed a result of excess area stored in membrane reservoirs such as microvilli, removal of the membrane reservoir should lead to an increase in $\tilde{K}_{A}$ up to $K_{A}$ of a bilayer in contact to a cortex. One conceivable experiment is the removal of cholesterol from the plasma membrane. Cholesterol depletion leads to dispersion of lipid rafts and therefore to removal of caveolae and microvilli acting as potential surface area reservoirs for the apical cell membrane [43, 144]. Immunostaining of ezrin and F-actin shows the reduction of these proteins during incubation with cholesterol depleting MBCD and AFMand SEM-imaging visualizes the disappearance of the membrane protrusions. This reduction in membrane material could also be quantified by monitoring time resolved changes in membrane capacitance, which are proportional to membrane area at the applied frequency, together with an increased endocytosis rate. As expected, the reduction in membrane area shifts the area compressibility modules to higher values, almost by factor of five. The strongest and most important finding is that the lower values attributed to areas with excess membrane disappear, while the larger area compressibility modulus most likely represents membrane regions without associated membrane reservoir (Table 9). The reduction of membrane reservoirs clearly results in a significant increase in membrane tension $\left(T_{0}\right.$ and $\left.T_{t}\right)$ by a factor of two.

Hence, we can attribute the lower $\tilde{K}_{A}$-values of untreated MDCK II cells, to areas on the cell membrane with no or little adhesive support and therefore accessibility to excess membrane area.

Tether pulling experiments are also used to obtain the accessibility to various mechanical parameters, which in addition characterize the plasma membrane of MDCK II cells. The 
membrane viscosity of the plasma membrane of MDCK II cells is estimated to be $\eta=$ $0.89 \cdot 10^{-6} \pm 0.18 \cdot 10^{-6} \mathrm{Ns} \mathrm{m}^{-1}$ [112]. For pure lipid bilayer values are found in the magnitude of $10^{-8}-10^{-6} \mathrm{Ns} \mathrm{m}^{-1}$ [138] [139]. The bending rigidity of the plasma membrane, always assumed to be in the order of $1 \cdot 10^{-19} \mathrm{Nm}$ (fluid lipid bilayer) [27], was determined to be $1.02 \cdot 10^{-19} \pm 0.65 \cdot 10^{-19} \mathrm{Nm}$ for living MDCK II cells. At last the radius of pulled tether $\left(R_{t}=12.4 \mathrm{~nm} \pm 3.9 \mathrm{~nm}\right)$, revealed a reasonable value for membrane tethers lying in the range of small vesicles also found in vivo [134] [140].

The above mentioned experiments clearly demonstrate the importance of the membranecytoskeleton adhesion for generating pre-stress in the plasma membrane controlled by the surface area regulation. Therefore, single cell experiments are devised to address the question to what extent the membrane-cortex-adhesion contributes to the mechanical behavior of one cell within a non-affected cell monolayer, mimicking the change of one cell upon genetic dysfunction. First the binding of the cytoskeleton to the membrane was inhibited by the microinjection of neomycin and secondly the binding sites for ezrin were increased by microinjecting $\mathrm{PIP}_{2}$. $\mathrm{PIP}_{2}$ inactivation led to considerable changes in the morphology of the apical membrane such as the disappearance of microvilli accompanied by a loss in cell height as compared to untreated adjacent cells. Besides, injected cells frequently develop tail-like protrusions and occasionally undergo apoptosis. Interestingly, the initial response of the cells directly after injection of neomycin provided evidence that prior to the collapse and straightening of the plasma membrane a decrease in the area compressibility modulus accompanied by an increase in tether lengths occurred indicative of a sudden availability of excess membrane area after removal of membrane cortex contacts. After this initial release of tension the cells compensated the loss of cross-linkers by removing excess area from the apical membrane [17] producing larger $\tilde{K}_{A}$ values along with an increase in membrane tension $\left(T_{0}\right.$ and $\left.T_{t}\right)$ by a factor of 2 due to disappearance of tension buffering membrane reservoirs. By injecting PIP $_{2}$ into the cytoplasm of the MDCK II cells an increased level of the phospholipid integrated into plasma membrane was detected by fluorescence microscopy confirming co-localization with the anchor-protein ezrin and the underlying cortical F-actin. Mechanical investigation revealed a significant increase in measured membrane tension by a factor of two. The apparent area compressibility modulus rise more than 10 times. This reflects a loss of excess membrane area, leading to a largely inextensible plasma membrane. Additionally, AFM images 
revealed a loss of microvilli and a more smoothed surface. A surprising observation was the recovery of initial tension values $3 \mathrm{~h}$ after microinjection of $\mathrm{PIP}_{2}$-micelles. Although, $\mathrm{PIP}_{2}$-micelle-injection and neomycin-injection lead to opposite bimolecular reactions the mechanical response is the same, a loss in measured excess membrane area (high $\tilde{K}_{A}$ values). However, cell stiffening due to $\mathrm{PIP}_{2}$-micelle injection is a reversible process. In case of microinjecting neomycin, the high apoptotic rate of injected cells display the importance of a dynamic cellular cortex and the major role of $\mathrm{PIP}_{2}$ in the interplay between plasma membrane and cytoskeleton governing the mechanical properties of the membrane. The manipulation of one single MDCK II cell inside an intact monolayer led to a mechanical change of the injected cell, but no alteration in the adjacent ones were observed.

The administration of osmotic stress is also a mechanical challenge to the plasma membrane. Osmotic pressure forces the cell to quickly adapt in order to avoid damages of the inextensible plasma membrane. Here, we could show that cells in the context of confluent monolayers adapt to hypoosmotic challenges in two ways. One quick, purely physical response is found relying entirely on the available membrane surface reservoirs and dissolution of the cytoskeleton preventing lysis of the plasma membrane and a second long-term response involving remodeling of the cytoskeleton-membrane junction and increased endocytosis to regain the initial tension. Cells increase their volume to avoid lysis by unfolding membrane from preexisting reserves [20], which is visible in the AFMtopological images by smoothening out membrane protrusions like microvilli during cell swelling at strong hypotonic treatment [199]. Mild hypotonic conditions do not visibly affect the integrity of the cortex. As a consequence, the apparent area compressibility modulus increases to respond to the increase in tension due to swelling. The increase in $\tilde{K}_{A}$ reflects a 2-fold loss of excess membrane due to membrane deformation, which is in accordance to calculated surface area increase by a factor of 1.7. The resulting change in volume compensates for the increased osmotic pressure. Tether pulling experiments reveal a drop in tension which we attribute predominately to a loss of actin-membrane contacts. Indentation experiments, however, show an increase in tension that is probably due to the additional osmotic pressure resisting indentation. This effect changes when subjecting the cells to stronger hypotonic stress. Strong hypoosmotic stress eventually leads to disintegration of the cortex and lift-off of the plasma membrane. Concomitantly, the 
apparent area compressibility modulus decreases indicative of allocation of excess membrane area from existing reservoirs. The loss of membrane-actin contact leads to strong swelling of the cell body and, as a consequence, tension drops since the cortex no longer supports the plasma membrane. A reorganization of the F-actin cytoskeleton is observed [200], which counteracts the external force [6]. This has a dramatic effect on membrane tension decreasing 1.5-fold. The disassembly of the F-actin cytoskeleton with cytochalasin D shows the same trend [130, 174, 175]. Over time, cortical tension is reestablished while excess membrane area is consumed by increased endocytosis, reflected by an increase in the apparent area compressibility modulus which is a necessary measure to restore the tension. This is confirmed by fluorescence micrographs displaying enhanced endocytosis. $\tilde{K}_{A}$-value after $6 \mathrm{~h}$ of regulation is known from lipid bilayer lacking membrane reservoirs or local region with strong connection to the underlying cytoskeleton. A stronger adhesion to the cortex is also detectable via tether pulling experiments displaying higher tether forces. Fluorescence microscopy revealed an enhanced ezrin distribution. Hence, confluent MDCK II cells are regulating their membrane tension and area during prolonged hypotonic exposure. The scenario of hypoosmotic challenges can therefore be envisioned as follows: under isotonic conditions the contractile and therefore tensed actomyosin cortex exerts an inward hydrostatic pressure on the cytosol, which gives rise to the spherical cap morphology of the MDCK II cells in a confluent monolayer. The tension of the cortex gives a rise to a pressure difference according to Laplace's law. As a consequence, membrane blebs devoid of a cortex usually occur at sites in which the cortex is damaged. Therefore, the cortex or better the actin-membrane contacts carry the tension. The (isotropic) tension of the plasma membrane produced by the contractile actomyosin therefore relies on molecular contacts between the actin cytoskeleton and the membrane. The apparent area compressibility modulus, which describes the membrane's resistance to in-plane stretching, mirrors excess area stored in protrusions and invaginations. The opposite is found for application of hypertonic stress. Tether forces increase due to inward osmotic pressure, while the tension inferred from indentation experiments is lower.

From the above mentioned experiments a comprehensive picture can be drawn emphasizing the close interplay between the membrane-cytoskeleton-adhesion and the mechanics of the plasma membrane. When cells are subjected to different mechanical challenges regarding the plasma membrane, specific regulating measures were taken by the 
cells either to restore tension inside the bilayer by reducing available membrane area or by degradation of the cytoskeleton to release tension in order to avoid membrane lysis. The adhesion of a suspended cell to a substrate and following spreading presuppose the availability of such dynamic membrane regulating processes. Therefore, this subject was investigated in the last part of this thesis. Cell shape changes during adhesion and spreading were taken into account by adjusting the geometrical constraints of the liquid droplet model from a sphere to a caped sphere. Time resolved mechanical probing of the cellular elasticity of the spreading process revealed that cell spreading requires a temporary release in tension to allow formation of membrane protrusions. Adhesion to the substrate leads to a partial rupturing of the cortical actin and a drop of tension along with a dramatic increase in the excess membrane area. In the course of spreading, membrane tension increases again by smoothing out existing membrane reservoirs as a result of tension homeostasis controlled by surface area regulation.

In conclusion, the mechanical properties of the plasma membrane of the epithelial cell line MDCK II were investigated using a comprehensive mechanical approach based on a tailored liquid droplet model. Subjecting the cells to various stimuli targeting membrane reservoir availability, actomyosin-integrity, membrane cytoskeleton attachment sites through injection of neomycin and $\mathrm{PIP}_{2}$ and hydrostatic pressure provoked a tension-driven response by adjusting the available surface area. The present work showed how confluent cells adapt to mechanically challenging situations as experienced during adhesion and spreading of a cell. These findings clearly show that the plasma membrane acts as a regulator for cellular deformation through tension homeostasis by exhaustion of membrane reservoirs. 


\section{References}

1. Suresh, S., Biomechanics and biophysics of cancer cells. Acta Mater., 2007. 55: p. 3989-4014.

2. Ingber, D.E., Mechanobiology and diseases of mechanotransduction. Ann. Med., 2003. 35(8): p. 564-577.

3. Sheetz, M.P. and J. Dai, Modulation of membrane dynamics and cell motility by membrane tension. Trends Cell Biol., 1996. 6(3): p. 85-89.

4. Raucher, D. and M.P. Sheetz, Membrane Expansion Increases Endocytosis Rate during Mitosis. J. Cell Biol., 1999. 144(3): p. 497-506.

5. Dai, J., H.P. Ting-Beall, and M.P. Sheetz, The Secretion-coupled Endocytosis Correlates with Membrane Tension Changes in RBL 2 H3 Cells. J. Gen. Physiol., 1997. 110(1): p. 1-10.

6. Apodaca, G., Modulation of membrane traffic by mechanical stimuli. Am. J. Physiol.-Renal Physiol., 2002. 282(2): p. 179-190.

7. Togo, T., T.B. Krasieva, and R.A. Steinhardt, A Decrease in Membrane Tension Precedes Successful Cell-Membrane Repair. Mol. Biol. Cell, 2000. 11(12): p. 4339-4346.

8. Gauthier, N.C., et al., Temporary increase in plasma membrane tension coordinates the activation of exocytosis and contraction during cell spreading. Proc. Natl. Acad. Sci., 2011. 108(35): p. 14467-14472.

9. Raucher, D. and M.P. Sheetz, Cell spreading and lamellipodial extension rate is regulated by membrane tension. J. Cell Biol., 2000. 148(1): p. 127-136.

10. Dai, J. and M.P. Sheetz, Membrane Tether Formation from Blebbing Cells. Biophys. J., 1999. 77(6): p. 3363-3370.

11. Algrain, M., et al., Ezrin contains cytoskeleton and membrane binding domains accounting for its proposed role as a membrane-cytoskeletal linker. J. Cell Biol., 1993. 120(1): p. 129-139.

12. Arpin, M., et al., Emerging role for ERM proteins in cell adhesion and migration. Cell Adhes. Migr., 2011. 5(2): p. 199-206.

13. Kaul, S.C., et al., Identification of a 55-kDa ezrin-related protein that induces cytoskeletal changes and localizes to the nucleolus. Exp. Cell Res., 1999. 250(1): p. 51-61. 
14. Saotome I., C.M., McClatchey A.I, Ezrin is essential for epithelial organization and villus morphogenesis in the developing intestine, in Dev. Cell, 62004. p. 855864.

15. Berryman, M., Z. Franck, and A. Bretscher, Ezrin is concentrated in the apical microvilli of a wide variety of epithelial cells whereas moesin is found primarily in endothelial cells. J. Cell Sci., 1993. 105(4): p. 1025-1043.

16. Bretscher A., E.K., Fehon RG., ERM proteins and merlin: integrators at the cell cortex, in Nat. Rev. Mol. Cell Biol.2002. p. 855-864.

17. Morris, C.E. and U. Homann, Cell surface area regulation and membrane tension. J. Membr. Biol., 2001. 179(2): p. 79-102.

18. Nassoy, P. and C. Lamaze, Stressing caveolae new role in cell mechanics. Trends Cell Biol., 2012. 22(7): p. 381-389.

19. Sinha, B., et al., Cells Respond to Mechanical Stress by Rapid Disassembly of Caveolae. Cell, 2011. 144(3): p. 402-413.

20. Kozera, L., E. White, and S. Calaghan, Caveolae act as membrane reserves which limit mechanosensitive I(Cl,swell) channel activation during swelling in the rat ventricular myocyte. PLoS One, 2009. 4(12): p. e8312.

21. Groulx, N., et al., Membrane Reserves and Hypotonic Cell Swelling. J. Membr. Biol., 2006. 214(1): p. 43-56.

22. Haston, W.S. and J.M. Shields, Contraction waves in lymphocyte locomotion. J. Cell Sci., 1984. 68(1): p. 227-241.

23. Kolega, J., Effects of mechanical tension on protrusive activity and microfilament and intermediate filament organization in an epidermal epithelium moving in culture. J. Cell Biol., 1986. 102(4): p. 1400-1411.

24. Rosenbluth, M.J., W.A. Lam, and D.A. Fletcher, Force microscopy of nonadherent cells: a comparison of leukemia cell deformability. Biophys J, 2006. 90(8): p. 29943003.

25. Sen, S., S. Subramanian, and D.E. Discher, Indentation and adhesive probing of a cell membrane with AFM: theoretical model and experiments. Biophys. J., 2005. 89(5): p. 3203-3213.

26. Goodsell, D.S., The Machinery of Life. 2nd ed ed2009: Springer.

27. Boal, D.H., Mechanics of the cell2002: Cambridge University Press. 
28. Ross, J.L., M.Y. Ali, and D.M. Warshaw, Cargo transport: molecular motors navigate a complex cytoskeleton. Curr. Opin. Cell Biol., 2008. 20(1): p. 41-47.

29. Bonifacino, J.S. and B.S. Glick, The Mechanisms of Vesicle Budding and Fusion. Cell, 2004. 116(2): p. 153-166.

30. Alberts, B., et al., Molecular Biology of the Cell, 4th edition2002, New York: Garland Science.

31. Boon, J.M. and B.D. Smith, Chemical control of phospholipid distribution across bilayer membranes. Med. Res. Rev., 2002. 22(3): p. 251-281.

32. Janmey, P.A. and P.K.J. Kinnunen, Biophysical properties of lipids and dynamic membranes. Trends Cell Biol., 2006. 16(10): p. 538-546.

33. Marshall, S.H. and G. Arenas, Antimicrobial peptides: A natural alternative to chemical antibiotics and a potential for applied biotechnology. Electron. J. Biotechnol., 2003. 6(2).

34. Cantley, L.C., The Phosphoinositide 3-Kinase Pathway. Science, 2002. 296(5573): p. $1655-1657$.

35. Czech, M.P., PIP2 and PIP3: Complex Roles at the Cell Surface. Cell, 2000. 100(6): p. 603-606.

36. Israelachvili, J.N., Intermolecular and Surface Forces. 3rd Edition ed2011: Academic Press.

37. Simons, K. and E. Ikonen, Functional rafts in cell membranes. Nature, 1997. 387(6633): p. 569-572.

38. Simons, K. and D. Toomre, Lipid rafts and signal transduction. Nat. Rev. Mol. Cell Biol., 2000. 1(1): p. 31-39.

39. Singer, S.J. and G.L. Nicolson, The Fluid Mosaic Model of the Structure of Cell Membranes. Science, 1972. 175(4023): p. 720-731.

40. Munro, S., Lipid Rafts: Elusive or Illusive? Cell, 2003. 115(4): p. 377-388.

41. Viola, A. and N. Gupta, Tether and trap: regulation of membrane-raft dynamics by actin-binding proteins. Nat. Rev. Immunol., 2007. 7(11): p. 889-896.

42. Pike, L.J., Rafts defined: a report on the Keystone symposium on lipid rafts and cell function. J. Lipid Res., 2006. 47(7): p. 1597-1598.

43. Pike, L.J. and J.M. Miller, Cholesterol Depletion Delocalizes Phosphatidylinositol Bisphosphate and Inhibits Hormone-stimulated Phosphatidylinositol Turnover. J. Biol. Chem., 1998. 273(35): p. 22298-22304. 
44. Caroni, P., New EMBO members' review: actin cytoskeleton regulation through modulation of PI(4,5)P(2) rafts. EMBO J, 2001. 20(16): p. 4332-6.

45. Kwik, J., et al., Membrane cholesterol, lateral mobility, and the phosphatidylinositol 4,5-bisphosphate-dependent organization of cell actin. Proc. Natl. Acad. Sci., 2003. 100(24): p. 13964-13969.

46. Yin, H.L. and P.A. Janmey, Phosphoinositide Regulation of the Actin Cytoskeleton. Annu. Rev. Physiol., 2003. 65(1): p. 761-789.

47. McLaughlin, S., et al., PIP(2) and proteins: interactions, organization, and information flow. Annu. Rev. Biophys. Biomol. Struct., 2002. 31: p. 151-175.

48. Fehon, R.G., A.I. McClatchey, and A. Bretscher, Organizing the cell cortex: the role of ERM proteins. Nat. Rev. Mol. Cell Biol., 2010. 11(4): p. 276-287.

49. Woodward, A.M. and D.H. Crouch, Cellular Distributions of the ERM Proteines in MDCK Epithelial Cells: Regulation by Growth and Cytoskeleal Integrity. Cell Biol. Int., 2001. 25: p. 205-213.

50. Gary, R. and A. Bretscher, Ezrin self-association involves binding of an N-terminal domain to a normally masked C-terminal domain that includes the F-actin binding site. Mol. Biol. Cell, 1995. 6(8): p. 1061-75.

51. Bretscher, A., D. Reczek, and M. Berryman, Ezrin: a protein requiring conformational activation to link microfilaments to the plasma membrane in the assembly of cell surface structures. J. Cell Sci., 1997. 110(24): p. 3011-3018.

52. Zhu, L., et al., High turnover of ezrin T567 phosphorylation: conformation, activity, and cellular function. Am J Physiol Cell Physiol, 2007. 293(3): p. C874C884.

53. Tsukita, S. and S. Yonemura, Cortical Actin Organization: Lessons from ERM (Ezrin/Radixin/Moesin) Proteins. J. Biol. Chem., 1999. 274(49): p. 34507-34510.

54. Gautreau, A., D. Louvard, and M. Arpin, ERM proteins and NF2 tumor suppressor: the Yin and Yang of cortical actin organization and cell growth signaling. Curr. Opin. Cell Biol., 2002. 14(1): p. 104-109.

55. Niggli, V., et al., Identification of a phosphatidylinositol-4,5-bisphosphate-binding domain in the N-terminal region of ezrin. FEBS Lett, 1995. 376(3): p. 172-176.

56. Roch, F., et al., Differential roles of PtdIns(4,5)P2 and phosphorylation in moesin activation during Drosophila development. J. Cell Sci., 2010. 123: p. 2058-2067. 
57. Hunter, K.W., Ezrin, a key component in tumor metastasis. Trends Mol. Med, 2004. 10(5): p. 201-204.

58. Huang, S. and D.E. Ingber, Cell tension, matrix mechanics, and cancer development. Cancer cell, 2005. 8(3): p. 175-176.

59. Winder, S.J. and K.R. Ayscough, Actin-binding proteins. J. Cell Sci., 2005. 118(4): p. 651-654.

60. Tanaka-Takiguchi, Y., et al., The elongation and contraction of actin bundles are induced by double-headed myosins in a motor concentration-dependent manner. J. Mol. Biol., 2004. 341(2): p. 467-476.

61. Caviston, J.P. and E.L.F. Holzbaur, Microtubule motors at the intersection of trafficking and transport. Trends Cell Biol., 2006. 16(10): p. 530-537.

62. Anitei, M. and B. Hoflack, Bridging membrane and cytoskeleton dynamics in the secretory and endocytic pathways. Nat. Cell. Biol., 2012. 14(1): p. 11-19.

63. Fuchs, E. and K. Weber, Intermediate Filaments: Structure, Dynamics, Function and Disease. Ann. Rev. Biochem., 1994. 63(1): p. 345-382.

64. Janmey, P.A., et al., Viscoelastic properties of vimentin compared with other filamentous biopolymer networks. J. Cell Biol., 1991. 113(1): p. 155-160.

65. Köster, D.V., Role of Caveolae in Membrane Tension, in Fakultät für Physik und Geowissenschaften2010, Universität Leipzig.

66. Helfrich, W., Elastic properties of lipid bilayers: theory and possible experiments. Zeitschrift für Naturforschung. Teil C: Biochemie, Biophysik, Biologie, Virologie, 1973. 28(11): p. 693-703.

67. Canham, P.B., The minimum energy of bending as a possible explanation of the biconcave shape of the human red blood cell. J. Theor. Biol., 1970. 26(1): p. 61-81.

68. Mohandas, N. and E. Evans, Mechanical Properties of the Red Cell Membrane in Relation to Molecular Structure and Genetic Defects. Annu. Rev. Biophys. Biomol. Struct., 1994. 23(1): p. 787-818.

69. Deserno, M., Fluid lipid membranes - a primer, 2007.

70. Rawicz, W., et al., Effect of chain length and unsaturation on elasticity of lipid bilayers. Biophys J, 2000. 79(1): p. 328-339.

71. Hamill, O.P. and B. Martinac, Molecular basis of mechanotransduction in living cells. Physiol. Rev., 2001. 81(2): p. 685-740. 
72. Lim, C.T., E.H. Zhou, and S.T. Quek, Mechanical models for living cells--a review. J. Biomech., 2006. 39(2): p. 195-216.

73. Cooke, B.M., N. Mohandas, and R.L. Coppel, The malaria-infected red blood cell: Structural and functional changes, in Adv. Parasitol.2001, Academic Press. p. 186.

74. Zhu, C., G. Bao, and N. Wang, Cell Mechanics: Mechanical Response, Cell Adhesion, and Molecular Deformation. Ann. Rev. Biomed. Eng., 2000. 2: p. 189226.

75. Crick, F.H.C. and A.F.W. Hughes, The physical properties of cytoplasm: A study by means of the magnetic particle method Part I. Experimental. Exp. Cell Res., 1950. 1(1): p. 37-80.

76. Mitchison, J.M. and M.M. Swann, The Mechanical Properties of the Cell Surface. J. Exp. Biol., 1954. 31(3): p. 443-460.

77. Rand, R.P. and A.C. Burton, Mechanical Properties of the Red Cell Membrane: I. Membrane Stiffness and Intracellular Pressure. Biophys. J., 1964. 4(2): p. 115-135.

78. Petersen, N.O., W.B. McConnaughey, and E.L. Elson, Dependence of locally measured cellular deformability on position on the cell, temperature, and cytochalasin B. Proc. Natl. Acad. Sci., 1982. 79(17): p. 5327-5331.

79. Geerts, H., et al., Nanovid tracking: a new automatic method for the study of mobility in living cells based on colloidal gold and video microscopy. Biophys. J., 1987. 52(5): p. 775-782.

80. Wang, N., J. Butler, and D. Ingber, Mechanotransduction across the cell surface and through the cytoskeleton. Science, 1993. 260(5111): p. 1124-1127.

81. Maksym, G.N., et al., Mechanical properties of cultured human airway smooth muscle cells from 0.05 to 0.4 Hz. J. Appl. Physiol., 2000. 89(4): p. 1619-1632.

82. Hoh, J.H. and C.A. Schoenenberger, Surface morphology and mechanical properties of MDCK monolayers by atomic force microscopy. J. Cell Sci., 1994. 107 ( Pt 5): p. 1105-1114.

83. Thoumine, O., O. Cardoso, and J.-J. Meister, Changes in the mechanical properties of fibroblasts during spreading: a micromanipulation study. Eur. Biophys. J, 1999. 28(3): p. 222-234. 
84. Hénon, S., et al., A New Determination of the Shear Modulus of the Human Erythrocyte Membrane Using Optical Tweezers. Biophys. J., 1999. 76(2): p. 11451151.

85. Mills, J.P., et al., Nonlinear Elastic and Viscoelastic Deformation of the Human Red Blood Cell with Optical Tweezers. Mol. Cell Biomec., 2004. 1(3): p. 169-180.

86. Weisenhorn, A.L., et al., Deformation and height anomaly of soft surfaces studied with an AFM. Nanotechnology, 1993. 4(2): p. 106.

87. Radmacher, M., Measuring the elastic properties of biological samples with the AFM. Eng. Med. Biol. Mag., 1997. 16(2): p. 47-57.

88. Rotsch, C., et al., AFM Imaging and elasticity measurments on living rat liver macrphages. Cell Biol. Int., 1997. 21(11): p. 685-696.

89. Hertz, H., Über die Berührung fester elastischer Körper. J. Reine Angew. Math., 1881. 92: p. 156-171.

90. Mathur, A.B., et al., Endothelial, cardiac muscle and skeletal muscle exhibit different viscous and elastic properties as determined by atomic force microscopy. J Biomech., 2001. 34(12): p. 1545-1553.

91. Kuznetsova, T.G., et al., Atomic force microscopy probing of cell elasticity. Micron, 2007. 38(8): p. 824-833.

92. Alonso, J.L. and W.H. Goldmann, Feeling the forces: atomic force microscopy in cell biology. Life Sci., 2003. 72(23): p. 2553-2560.

93. Sneddon, I.N., The Relation between load and Penetration in the axisymmetric boussinesq Problem for a Punch of arbitarary Profile. Int. J. Eng. Sci., 1965. 3: p. 47-57.

94. Briscoe, B.J., K.S. Sebastian, and M.J. Adams, The effect of indenter geometry on the elastic response to indentation. J. Phys. D: Appl. Phys., 1994. 27(6): p. 1156.

95. Costa, K.D. and F.C.P. Yin, Analysis of Indentation: Implications for Measuring Mechanical Properties With Atomic Force Microscopy. J. BIOMECH. ENG-T ASME, 1999. 121(5): p. 462-471.

96. Lin, D.C., E.K. Dimitriadis, and F. Horkay, Robust Strategies for Automated AFM Force Curve Analysis---II: Adhesion-Influenced Indentation of Soft, Elastic Materials. J. BIOMECH. ENG-T ASME, 2007. 129(6): p. 904-912.

97. Domke, J. and M. Radmacher, Measuring the Elastic Properties of Thin Polymer Films with the Atomic Force Microscope. Langmuir, 1998. 14(12): p. 3320-3325. 
98. Yeung, A. and E. Evans, Cortical shell-liquid core model for passive flow of liquidlike spherical cells into micropipets. Biophys. J., 1989. 56(1): p. 139-149.

99. Hochmuth, R.M., Cell and Membrane Biomechanics: Class 4: Liquid Cell, Law of Laplace, Cortical Tension, Liquid Drop Model, 1999.

100. Lomakina, E.B., et al., Rheological analysis and measurement of neutrophil indentation. Biophys. J., 2004. 87(6): p. 4246-4258.

101. Fery, A. and R. Weinkamer, Mechanical properties of micro- and nanocapsules: Single-capsule measurements. Polymer, 2007. 48(25): p. 7221-7235.

102. Janshoff, A., Cell indentation by atomic force microscopy - an extended liquid drop model, 2012, Private Communication: Göttingen.

103. Waugh, R.E. and R.M. Hochmuth, Mechanical equilibrium of thick, hollow, liquid membrane cylinders. Biophys. J., 1987. 52(3): p. 391-400.

104. Evans, E.A. and R. Skalak, Mechanics and Thermodynamic of Biomembranes: Pt. $1,1979$.

105. Hochmuth, F.M., et al., Deformation and flow of membrane into tethers extracted from neuronal growth cones. Biophys. J., 1996. 70(1): p. 358-369.

106. Hochmuth, R.M. and W.D. Marcus, Membrane tethers formed from blood cells with available area and determination of their adhesion energy. Biophys. J., 2002. 82(6): p. 2964-2969.

107. Hochmuth, R.M., et al., Extensional flow of erythrocyte membrane from cell body to elastic tether. II. Experiment. Biophys. J., 1982. 39(1): p. 83-89.

108. Heinrich, V. and R. Waugh, A piconewton force transducer and its application to measurement of the bending stiffness of phospholipid membranes. Ann. Biomed. Eng., 1996. 24(5): p. 595-605.

109. Waugh, R.E., Surface viscosity measurements from large bilayer vesicle tether formation. I. Analysis. Biophys. J., 1982. 38(1): p. 19-27.

110. Brochard-Wyart, F., et al., Hydrodynamic narrowing of tubes extruded from cells. Proc. Natl. Acad. Sci., 2006. 103(20): p. 7660-7663.

111. Krieg, M., et al., Tensile forces govern germ-layer organization in zebrafish. Nat. Cell Biol., 2008. 10(4): p. 429-36.

112. Sun, M., et al., The effect of cellular cholesterol on membrane-cytoskeleton adhesion. J. Cell Sci., 2007. 120(Pt 13): p. 2223-2231. 
113. Hochmuth, R., et al., Mechanical measurement of red cell membrane thickness. Science, 1983. 220(4592): p. 101-102.

114. Dai, J. and M.P. Sheetz, Mechanical properties of neuronal growth cone membranes studied by tether formation with laser optical tweezers. Biophys. J., 1995. 68(3): p. 988-996.

115. Zhang, Y. and L.C. Yu, Microinjection as a tool of mechanical delivery. Curr. Opin. Biotechnol., 2008. 19(5): p. 506-10.

116. Hedberg, K.M., et al., PDGF and neomycin induce similar changes in the actin cytoskeleton in human fibroblasts. Cell Motil. Cytoskeleton, 1993. 24(2): p. 139149.

117. Zhang, H., et al., Synthesis and Biological Activity of Phospholipase C-Resistant Analogues of Phosphatidylinositol 4,5-bisphosphate. Journal of the American Chemical Society, 2006. 128(17): p. 5642-5643.

118. Barenholz, Y., et al., A simple method for the preparation of homogeneous phospholipid vesicles. Biochemistry, 1977. 16(12): p. 2806-10.

119. Binnig, G., C.F. Quate, and C. Gerber, Atomic Force Microscope. Phys. Rev. Lett., 1986. 56(9): p. 930-933.

120. Fujisawa, S., et al., Difference between the forces measured by an optical lever deflection and by an optical interferometer in an atomic force microscope. Rev. Sci. Instrum., 1994. 65(3): p. 644-647.

121. Butt, H.-J., Capella Brunero Kappl Michael, Force measurements with the atomic force microscope: Technique, interpretation and applications. Surf. Sci. Rep., 2005. 59: p. 1-152.

122. Radmacher, M., et al., Measuring the viscoelastic properties of human platelets with the atomic force microscope. Biophys. J., 1996. 70(1): p. 556-67.

123. Haga, H., et al., Elasticity mapping of living fibroblasts by AFM and immunofluorescence observation of the cytoskeleton. Ultramicroscopy, 2000. 82(14): p. 253-8.

124. Sheetz, M.P., Cell control by membrane-cytoskeleton adhesion. Nat. Rev. Mol. Cell Biol., 2001. 2(5): p. 392-6.

125. Wegerhoff, R., O. Weidlich, and M. Kässens, Basics of Light Microscopy \& Imaging: GIT VERLAG GmbH \& Co. KG. 
126. Giaever, I. and C.R. Keese, A morphological biosensor for mammalian cells. Nature, 1993. 366(6455): p. 591-2.

127. Xiao, C. and J.H.T. Luong, On-line monitoring of cell growth and cytotoxicity using electric cell-substrate impedance sensing (ECIS). Biotechnol. Prog., 2003. 19(3): p. 1000-1005.

128. Lo, C.M., C.R. Keese, and I. Giaever, Impedance analysis of MDCK cells measured by electric cell-substrate impedance sensing. Biophys. J., 1995. 69(6): p. 2800-2807.

129. Lo, C.-M. and J. Ferrier, Impedance analysis of fibroblastic cell layers measured by electric cell-substrate impedance sensing. Phys. Rev. E, 1998. 57: p. 6982-6987.

130. Steltenkamp, S., et al., Membrane Stiffness of Animal Cells Challenged by Osmotic Stress. Small, 2006. 2(8-9): p. 1016-1020.

131. Rawicz, W., et al., Elasticity, strength, and water permeability of bilayers that contain raft microdomain-forming lipids. Biophys. J., 2008. 94(12): p. 4725-4736.

132. Butor, C. and J. Davoust, Apical to basolateral surface area ratio and polarity of MDCK cells grown on different supports. Exp. Cell Res., 1992. 203(1): p. 115-127.

133. Bo, L. and R.E. Waugh, Determination of bilayer membrane bending stiffness by tether formation from giant, thin-walled vesicles. Biophys. J., 1989. 55(3): p. 509517.

134. Reviakine, I. and A. Brisson, Formation of Supported Phospholipid Bilayers from Unilamellar Vesicles Investigated by Atomic Force Microscopy. Langmuir, 2000. 16: p. 1806-1815.

135. Shao, J.Y. and R.M. Hochmuth, Micropipette suction for measuring piconewton forces of adhesion and tether formation from neutrophil membranes. Biophys. J., 1996. 71(5): p. 2892-2901.

136. Evans, E.A., R. Waugh, and M. L., Elastic area compressibility modulus of red cell membrane Biophys. J., 1976. 16: p. 585-595.

137. Daily, B., E.L. Elson, and G.I. Zahalak, Cell poking. Determination of the elastic area compressibility modulus of the erythrocyte membrane. Biophys. J., 1984. 45(4): p. 671-82.

138. Mabrouk, E., et al., Formation and material properties of giant liquid crystal polymersomes. Soft Matter, 2009. 5(9): p. 1870-1878. 
139. Evans, E.A. and R.M. Hochmuth, Membrane viscoelasticity. Biophys J, 1976. 16(1): p. 1-11.

140. Castorph, S., et al., Synaptic vesicles studied by dynamic light scattering. Eur. Phys. J. E Soft Matter, 2011. 34(6): p. 1-11.

141. Zidovetzki, R. and I. Levitan, Use of cyclodextrins to manipulate plasma membrane cholesterol content: Evidence, misconceptions and control strategies. Biochim. Biophys. Acta-Biomembr., 2007. 1768(6): p. 1311-1324.

142. Poole, K., et al., The effect of raft lipid depletion on microvilli formation in MDCK cells, visualized by atomic force microscopy. FEBS Lett., 2004. 565(1-3): p. 53-58.

143. Dreja, K., et al., Cholesterol Depletion Disrupts Caveolae and Differentially Impairs Agonist-Induced Arterial Contraction. Arterioscler. Thromb. Vasc. Biol., 2002. 22(8): p. 1267-1272.

144. Edidin, M., The state of lipid rafts: from model membranes to cells. Annu. Rev. Biophys. Biomol. Struct., 2003. 32: p. 257-283.

145. Kataoka, N., et al., Measurements of endothelial cell-to-cell and cell-to-substrate gaps and micromechanical properties of endothelial cells during monocyte adhesion. Proc. Natl. Acad. Sci., 2002. 99(24): p. 15638-15643.

146. Francis, S.A. and et al., Rapid reduction of MDCK cell cholesterol by methyl-betacyclodextrin alters steady state transepithellial electrical resistance. Eur. J. Cell Biol., 1999. 78: p. 473-484.

147. Huttenlocher, A. and A.R. Horwitz, Integrins in Cell Migration. Cold Spring Harb Perspect. Biol., 2011. 3(9).

148. Byfield, F.J., et al., Cholesterol Depletion Increases Membrane Stiffness of Aortic Endothelial Cells. Biophys. J., 2004. 87(5): p. 3336-3343.

149. Sens, P. and M.S. Turner, Budded membrane microdomains as tension regulators. Phys. Rev. E, 2006. 73(3 Pt 1): p. 031918.

150. Bretscher, A., Microfilament Structure and Function in the Cortical Cytoskeleton. Annu. Rev. Cell Biol., 1991. 7(1): p. 337-374.

151. Janke, M., et al., Actin binding of ezrin is activated by specific recognition of PIP2functionalized lipid bilayers. Biochemistry, 2008. 47(12): p. 3762-3769.

152. Bosk, S., et al., Activation of F-Actin Binding Capacity of Ezrin: Synergism of PIP2 Interaction and Phosphorylation. Biophys. J., 2011. 100(7): p. 1708-1717. 
153. Harder, T., et al., Specific release of membrane-bound annexin II and cortical cytoskeletal elements by sequestration of membrane cholesterol. Mol. Biol. Cell, 1997. 8(3): p. 533-45.

154. Limouze, J., et al., Specificity of blebbistatin, an inhibitor of myosin II. J. Muscle Res. Cell Motil., 2004. 25(4): p. 337-341.

155. Tinevez, J.-Y., et al., Role of cortical tension in bleb growth. Proc. Natl. Acad. Sci., 2009. 106(44): p. 18581-18586.

156. Cunningham, C.C., Actin polymerization and intracellular solvent flow in cell surface blebbing. J. Cell Biol., 1995. 129(6): p. 1589-1599.

157. Rotsch, C. and M. Radmacher, Drug-induced changes of cytoskeletal structure and mechanics in fibroblasts: an atomic force microscopy study. Biophys. J., 2000. 78(1): p. 520-535.

158. Raucher, D. and M.P. Sheetz, Characteristics of a membrane reservoir buffering membrane tension. Biophys. J., 1999. 77(4): p. 1992-2002.

159. Castillo, A., et al., Myosin II-actin interaction in MDCK cells: role in cell shape changes in response to Ca2+ variations. J. Muscle. Res. Cell Motil., 1998. 19(5): p. 557-574.

160. Sutton, T.A., H.E. Mang, and S.J. Atkinson, Rho-kinase regulates myosin II activation in MDCK cells during recovery after ATP depletion. Am. J. Physiol. RENAL, 2001. 281(5): p. F810-F818.

161. Martens, J. and M. Radmacher, Softening of the actin cytoskeleton by inhibition of myosin II. Pflugers Arch., 2008. 456(1): p. 95-100.

162. Kerr, J.F.R., A.H. Wyllie, and A.R. Currie, Apoptosis: A Basic Biological Phenomenon with Wideranging Implications in Tissue Kinetics Brit. J. Cancer, 1972. 26.

163. Golebiewska, U., et al., Diffusion Coefficient of Fluorescent Phosphatidylinositol 4,5-bisphosphate in the Plasma Membrane of Cells. Mol. Biol. Cell, 2008. 19(4): p. 1663-1669.

164. Martin, T.F., PI(4,5)P(2) regulation of surface membrane traffic. Curr. Opin. Cell Biol., 2001. 13(4): p. 493-9.

165. Simonsen, A., et al., The role of phosphoinositides in membrane transport. Curr. Opin. Cell Biol., 2001. 13(4): p. 485-492. 
166. Fine, T. and et al., Elasticity mapping of apical cell membranes. Soft Matter, 2009. 5: p. 3262-3265.

167. Kocun, M. and A. Janshoff, Pulling Tethers from Pore-Spanning Bilayers: Towards Simultaneous Determination of Local Bending Modulus and Lateral Tension of Membranes. Small, 2012. 8(6): p. 847-851.

168. Komaragiri, U., Begley M.R. and J.G. Simmonds, The mechanical response of freestanding circular elastic films under point and pressure loads. J. Appl. Mech., 2005. 72: p. 203-212.

169. Lee, C., et al., Measurement of the elastic properties and intrinsic strength of monolayer graphene. Science, 2008. 321(5887): p. 385-388.

170. Krieg, M., et al., A bond for a lifetime: employing membrane nanotubes from living cells to determine receptor-ligand kinetics. Angew. Chem.-Int. Edit., 2008. 47(50): p. $9775-7$.

171. Yoneda, M. and K. Dan, Tension at the Surface of the Dividing Sea-Urchin Egg. J. Cell Biol., 1972. 57(3): p. 575-587.

172. Dubin-Thaler, B.J., et al., Nanometer Analysis of Cell Spreading on Matrix-Coated Surfaces Reveals Two Distinct Cell States and STEPs. Biophys. J., 2004. 86(3): p. 1794-1806.

173. Keren, K., Membrane tension leads the way. Proc. Natl. Acad. Sci., 2011. 108(35): p. $14379-14380$.

174. Guilak, F., G.R. Erickson, and H.P. Ting-Beall, The effects of osmotic stress on the viscoelastic and physical properties of articular chondrocytes. Biophys. J., 2002. 82(2): p. $720-727$.

175. Spagnoli, C., et al., Atomic force microscopy analysis of cell volume regulation. Phys. Rev. E Stat. Nonlin. Soft Matter Phys., 2008. 78(3 Pt 1): p. 031916.

176. Kiesel, M., et al., Swelling-Activated Pathways in Human T-Lymphocytes Studied by Cell Volumetry and Electrorotation. Biophys. J., 2006. 90(12): p. 4720-4729.

177. Zhou, E.H., et al., Universal behavior of the osmotically compressed cell and its analogy to the colloidal glass transition. Proc. Natl. Acad. Sci., 2009. 106(26): p. 10632-10637.

178. Youhua, T., et al., Mechanical Characterization of Human Red Blood Cells Under Different Osmotic Conditions by Robotic Manipulation With Optical Tweezers. IEEE Trans. Biomed. Eng., 2010. 57(7): p. 1816-1825. 
179. Ting-Beall, H., D. Needham, and R. Hochmuth, Volume and osmotic properties of human neutrophils. Blood, 1993. 81(10): p. 2774-2780.

180. Sahai, E. and C.J. Marshall, Differing modes of tumour cell invasion have distinct requirements for Rho/ROCK signalling and extracellular proteolysis. Nat. Cell. Biol., 2003. 5(8): p. 711-9.

181. Mills, J.C., et al., Apoptotic Membrane Blebbing Is Regulated by Myosin Light Chain Phosphorylation. The Journal of Cell Biology, 1998. 140(3): p. 627-636.

182. Paluch, E., et al., Dynamic modes of the cortical actomyosin gel during cell locomotion and division. Trends Cell Biol., 2006. 16(1): p. 5-10.

183. Norman, L.L., et al., Cell Blebbing and Membrane Area Homeostasis in Spreading and Retracting Cells. Biophys J, 2010. 99(6): p. 1726-1733.

184. Gauthier, N.C., et al., Plasma Membrane Area Increases with Spread Area by Exocytosis of a GPI-anchored Protein Compartment. Molecular Biology of the Cell, 2009. 20(14): p. 3261-3272.

185. Wakatsuki, T., R.B. Wysolmerski, and E.L. Elson, Mechanics of cell spreading: role of myosin II. Journal of Cell Science, 2003. 116(8): p. 1617-1625.

186. Bereiter-Hahn, J., et al., Spreading of trypsinized cells: cytoskeletal dynamics and energy requirements. J. Cell Sci., 1990. 96(1): p. 171-188.

187. Mazerik, J.N. and M.J. Tyska, Myosin-1a targets to microvilli using multiple membrane binding motifs in the tail homology 1 (TH1) domain. J. Biol. Chem., 2012.

188. Charras, G.T., et al., Reassembly of contractile actin cortex in cell blebs. J. Cell Biol., 2006. 175(3): p. 477-490.

189. Taylor, A.C., Attachment and spreading of cells in culture. Exp. Cell. Res., 1961. 8, Supplement(0): p. 154-173.

190. Keller, H. and P. Eggli, Protrusive activity, cytoplasmic compartmentalization, and restriction rings in locomoting blebbing Walker carcinosarcoma cells are related to detachment of cortical actin from the plasma membrane. Cell Motil. Cytoskel., 1998. 41(2): p. 181-193.

191. Burridge, K. and K. Wennerberg, Rho and Rac Take Center Stage. Cell, 2004. 116(2): p. 167-179. 
192. Arthur, W.T. and K. Burridge, RhoA Inactivation by p190RhoGAP Regulates Cell Spreading and Migration by Promoting Membrane Protrusion and Polarity. Mol. Biol. Cell, 2001. 12(9): p. 2711-2720.

193. Ren, X.-D., W.B. Kiosses, and M. Alexander Schwartz, Regulation of the small GTP-binding protein Rho by cell adhesion and the cytoskeleton. EMBO J, 1999. 18(3): p. 578-585.

194. Ridley, A.J. and A. Hall, The small GTP-binding protein rho regulates the assembly of focal adhesions and actin stress fibers in response to growth factors. Cell, 1992. 70(3): p. 389-399.

195. Barry, S.T., et al., Requirement for Rho in Integrin Signalling. Cell Comm. Adhes., 1997. 4(6): p. 387-398.

196. Worthylake, R.A., et al., RhoA is required for monocyte tail retraction during transendothelial migration. J. Cell Biol., 2001. 154(1): p. 147-160.

197. Worthylake, R.A. and K. Burridge, RhoA and ROCK Promote Migration by Limiting Membrane Protrusions. J. Biol. Chem., 2003. 278(15): p. 13578-13584.

198. Rauvala, H., W.G. Carter, and S.I. Hakomori, Studies on cell adhesion and recognition. I. Extent and specificity of cell adhesion triggered by carbohydratereactive proteins (glycosidases and lectins) and by fibronectin. J. Cell Biol., 1981. 88(1): p. 127-137.

199. Cornet, M., Y. Isobe, and L.F. Lemanski, Effects of anisosmotic conditions on the cytoskeletal architecture of cultured PC12 cells. J. Morphol., 1994. 222(3): p. 269286.

200. Cornet, M., E. Delpire, and R. Gilles, Study of microfilaments network during volume regulation process of cultured PC 12 cells. Pflugers Arch., 1987. 410(1): p. 223-225. 
List of Abbreviations

MDCK II

AFM

$\mathrm{PIP}_{2}$

ATP

CMC

ERM

FERM

ERMAD

$\kappa$

E

$L_{p}$

F

$R_{C}$

$v$

$\delta$

$P_{i}$

$P_{o}$

T

$K_{A}$

$\tilde{K}_{A}$

$T_{0}$

$T_{t}$

$k_{B} T$

$E_{\text {shear }}$
Mardin-Darbin canine kidney (cells)

Atomic Force Microscope

Phosphatidylinositol(4,5)-bisphosphate

Adenosine-5'-triphosphate

Critical micelle concentration

Ezrin, radixin, moesin

Four-point one ezrin, radixin, moesin

ERM associated domain

Bending rigidity

Young's modulus

Persistence length

Force

Cell radius

Poisson's ration

Indentation depth

Pressure inside the cell

Pressure outside the cell

Tension

Area compressibility modulus

Apparent area compressibility modulus

Pre-tension (indentation)

In-plane tension (tether pulling)

Boltzmann constant times temperature

Shear energy 


\begin{tabular}{|c|c|}
\hline$E_{\text {bend }}$ & Bending energy \\
\hline$E_{\text {strech }}$ & Stretching energy \\
\hline$A$ & Surface of a cell \\
\hline$A_{0}$ & Initial surface of the cell \\
\hline$A_{e x}$ & Excess surface area \\
\hline$R_{i}$ & Radius of contact zone \\
\hline$\phi$ & Wetting angel \\
\hline$C_{1}, C_{2}$ & Mean curvatures \\
\hline$V$ & Volume \\
\hline$F_{\text {tether }}$ & Tether force \\
\hline$\eta$ & Membrane viscosity \\
\hline$R_{t}$ & Tether radius \\
\hline$k$ & Spring constant of cantilever \\
\hline$d$ & Cantilever deflection \\
\hline$\alpha$ & Current flowing under the cells in the adhesion zone \\
\hline$R_{b}$ & Barrier resistance \\
\hline$C_{m}$ & Specific capacity of the plasma membrane \\
\hline MBCD & Methyl- $\beta$-cyclo-dextrin \\
\hline$W$ & Cell width \\
\hline$\rho$ & Specific extracellular medium resistivity \\
\hline
\end{tabular}




\section{List of Figures}

Figure 1: Schematic drawing of a eukaryotic cell.

Figure 2: A: Calotte model overlaid with chemical structure of a phospholipid, highlighted are head and tail group to emphasize amphiphilic character. B: Chemical structure of most common alcohols building the hydrophilic head of a lipid. C: Packing parameter $p$ predicts the structure of the aggregates formed from lipids placed in aqueous solution.

Figure 3: Chemical structure of cholesterol A: Calotte model. B: Structural formula.

Figure 4: Domain organization of the protein ezrin.

Figure 5: Schematic illustration of ezrin binding to the plasma membrane and the F-actin cytoskeleton.

Figure 6: Structure and arrangement of the biopolymer actin. A: Schematic illustration of the ATP driven Actin polymerization. B: Schematic drawing of actin structure within the cell. C: F-actin staining of subconfluent MDCK II cells. D: F-actin staining of an adhering and spreading MDCK II cell.

Figure 7: A: Subunit of microtubules is a heterodimer, a linked pair of $\alpha$ - and $\beta$ tubulin. B: Structure and arrangement of microtubules inside a cell. C: Fluorescence micrograph of immustaining of microtubules of confluent MDCK II cells. D: Fluorescence staining of the nucleus (blue) and the microtubules (green) during cell division.

Figure 8: A: Drawing of the structure of an intermediate filament. B: Schematic illustration of the arrangement of intermediate filaments inside a cell.

Figure 9: Illustration of membrane deformation. A: In-plane shearing. B: In-plane stretching. C: Bending results in an out of plane deformation described by its two curvatures $C_{1}$ and $C_{2}$. Modified from [65].

Figure 10: Illustration of Young's modules of different materials from soft to hard. Modified from [92].

Figure 11: Both conical and pyramidal tips are shown, together with a blunt tip. $a$ is the contact radius. $\alpha$ is the half-opening angle of cone or pyramid. $b$ is the radius at which the tapered sides transition into a spherical tip of radius $R$. Transition from blunt tip to sharp tip, when $R=b=0$, modified from [94].

Figure 12: Force distance trace curve recorded on a MDCK II cell (dots). In red, the Hertz-model is fitted to experimental data.

Figure 13: Force balance in a sphere with constant surface tension. 
Figure 14: Illustration of a conically shaped probe indenting a spherical, nonadhering cell. $P_{i}, P_{o}$ and $P$ are the pressure of the cell interior, fluid surrounding the cell, and tip. The opening angle of the cone is $\alpha . R_{c}$ is the radius of the cell.

Figure 15: Representative force-distance trace curve (black line) is used for determine the area compressibility modulus $\tilde{K}_{A}$ and tension $T_{0}$ using a modified liquid droplet model for indentation experiments fitted to the experimental data (red dots).

Figure 16: The cell is described by a spherical cap. $R_{1}$ is the base radius, $r_{1}$ the radial position beyond which the membrane is not in contact with the tip. $\phi_{0}$ the contact angle between cell and substrate, $\delta$ the indentation depth and h the cell height before indentation.

Figure 17: Simulation of a spherical cap representing the apex of a confluent MDCK II cell.

Figure 18: Force distance curve recorded on a confluent MDCK II cell (black). Red squares represent a simulated force distance curve for a modeled apex with $R_{0}=35 \mu \mathrm{m}$ and a contact angle of $\phi=20^{\circ}$. Mechanical parameters are set to values of: pre-tension $T_{0}=0.3 \mathrm{mN} \mathrm{m}^{-1}$ and $\tilde{K}_{A}=0.08 \mathrm{~N} \mathrm{~m}^{-1}$.

Figure 19: Typical force indentation curves according to the liquid droplet model. A: Simulated force curves assuming an area compressibility of $\tilde{K}_{\mathrm{A}}=0.2 \mathrm{~N} \mathrm{~m}^{-1}$

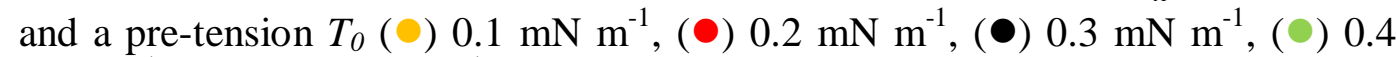
$\mathrm{mN} \mathrm{m}^{-1}$, (๑) $0.5 \mathrm{mN} \mathrm{m}^{-1}$. B: Contour of an adherent cell represented by a spherical cap subject to indentation using a conical indenter and a maximal force of $1 \mathrm{nN}$. $T_{0}$ and $\tilde{K}_{A}$ are set to $0.3 \mathrm{mN} \mathrm{m}^{-1}$ and (॰) $0.05 \mathrm{~N} \mathrm{~m}^{-1},(\bullet) 0.4 \mathrm{~N} \mathrm{~m}^{-1}$. C: Variation of simulated force curves assuming a pre-tension of $T_{0}=0.3 \mathrm{mN} \mathrm{m}^{-1}$ and area compressibility $\tilde{K}_{A}(\odot) 0.05 \mathrm{~N} \mathrm{~m}^{-1},(\bullet) 0.1 \mathrm{~N} \mathrm{~m}^{-1},(\bullet) 0.2 \mathrm{~N} \mathrm{~m}^{-1}$, (॰) 0.3 $\mathrm{N} \mathrm{m}^{-1}$, (॰) $0.4 \mathrm{~N} \mathrm{~m}^{-1}$.D: Contour of an adherent cell represented by a spherical cap subject to indentation using a conical indenter and a maximal force of $1 \mathrm{nN}$. $\tilde{K}_{A}$ and $T_{0}$ are set to $0.2 \mathrm{~N} \mathrm{~m}^{-1}$ and (०) $0.1 \mathrm{mN} \mathrm{m}^{-1}$, (०) $0.5 \mathrm{mN} \mathrm{m}^{-1}$. A initial cell radius $R_{0}=35 \mu \mathrm{m}$ (note that $R_{1}=R_{0} \sin (\phi)$ ), a wetting angle prior to indentation $\phi$ $=20^{\circ}$, a half opening angle of the conical indenter of $\alpha=17.5^{\circ}$ are used for computation.

Figure 20: Influence of cellular shape (wetting angle) on mechanical parameter. A: Contour of an adherent cell represented by a spherical cap subject to indentation using a conical indenter and a maximal force of $1 \mathrm{nN}$. A initial cell radius $R_{0}=35 \mu \mathrm{m}$ (note that $R_{1}=R_{0} \sin (\phi)$ ), a wetting angle prior to indentation $\phi$ $=(-) 16^{\circ},(-) 20^{\circ},(-) 60^{\circ}$, a half opening angle of the conical indenter of $\alpha=17.5$ ${ }^{\circ}$ are used for computation. B: Simulated force curves assuming a wetting angle prior to indentation $\phi=(\bullet) 16^{\circ},(\odot) 18^{\circ},(\bullet) 20^{\circ},(\odot) 25^{\circ},(\odot) 30^{\circ},(\odot) 40^{\circ}$, (॰) $50^{\circ},\left(^{\circ}\right) 60^{\circ} . \mathrm{C}: \tilde{K}_{A}$ increases with growing wetting angle, while $T_{0}$ stays almost constant. 
Figure 21: Influence of cellular shape (cell radius) on mechanical parameter. A: Contour of an adherent cell represented by a spherical cap subject to indentation using a conical indenter and a maximal force of $1 \mathrm{nN}$. A wetting angle prior to indentation of $\phi=20^{\circ}$ and an initial cell radius $R_{0}=(-) 20 \mu \mathrm{m},(-) 35 \mu \mathrm{m},(-) 60$ $\mu \mathrm{m}$, (note that $R_{1}=R_{0} \sin (\phi)$ ). A half opening angle of the conical indenter of $\alpha=$ $17.5^{\circ}$ are used for computation. B: Simulated force curves assuming an initial cell radius $R_{0}=(\bullet) 20 \mu \mathrm{m},(\bullet) 25 \mu \mathrm{m},(\bullet) 30 \mu \mathrm{m},(\bullet) 35 \mu \mathrm{m},(\bullet) 40 \mu \mathrm{m},(\bullet) 45$ $\mu \mathrm{m},(\odot) 50 \mu \mathrm{m},(\odot) 45 \mu \mathrm{m}$. C: While increasing the wetting angle $\phi$ prior to indentation, the initial cell radius $R_{0}$ also increases. This leads to an increase in $\tilde{K}_{A}$ , while $T_{0}$ stays almost constant.

Figure 22: A: Schematic illustration of formation of a membrane tether by pulling a AFM tip from the surface. Membrane shows adhesion to the underlying cortex and membrane reservoirs B: Retrace curve in blue displays formation of membrane tethers (force plateau) employed to determine tension $T_{t}$ from Equation 24.

Figure 23: Semi-automatic microinjection. The injection level has to be defined by slight pressing on the cell membrane. After setting the level, the capillary is moved back to the search level. Modified from Brochure InjectMan NI 2 "Injection meets Innovation".

Figure 24: (A) Phase contrast micrograph of MDCK II cell during microinjection. Injected cell blows up and loses contrast. Scale bar is $10 \mu \mathrm{m}$. (B) Fluorescence micrograph of FITC-dextran filled glass capillary.

Figure 25: Evaluation of the quality of the performed microinjection. A: Fluorescence micrograph of a propidium-iodide-staining of FITC-dextran injected cells (B) high injection parameter $P_{i}=150 \mathrm{hPa}$ and $t_{i}=0.5 \mathrm{~s}$. Scale bar is $20 \mu \mathrm{m}$. Red nuclei display loss of membrane integrity. C: Red staining of the nuclei is missing for injection parameter of $P_{i}=60 \mathrm{hPa}$ and $t_{i}=0.3 \mathrm{~s}$ (D). Scale bar is 20 $\mu \mathrm{m}$.

Figure 26: MDCK II cells display blebs after microinjection. A: Phase contrast micrograph. Arrow display blebs. Scale bar $10 \mu \mathrm{m}$. B: Fluorescence micrograph of FITC-dextran injected cells. Huge blebs are only visible in the fluorescence image.

Figure 27: Subconfluent MDCK II cells are imaged with the AFM after microinjection. A: AFM height image. Arrows indicate structural changes of injected cell (upper arrow) and unsuccessfully microinjected cell (lower arrow). B: AFM deflection image showing details of the surface, like stress fibers and plasma membrane protrusions. C: Fluorescence micrograph of FITC-dextran injected cells. D: Phase contrast micrograph of the same position as in A, B, C.

Figure 28: Illustration of an indentation experiment on a hard surface required for data processing. A: Cantilever is moved in the direction of the sample by a certain distance (height measured) and is bend in the opposite direction $(d)$ while the sample is indented by $\delta$. B: Force curve in its raw form: detector signal $(\mathrm{mV})$ versus piezo displacement $(\mathrm{nm})$. C: After determine the sensitivity of the system 
and calculating the spring constant $k$, the force distance curve (black) is obtained. Dashed blue curve represents force versus height measured.

Figure 29: Force mapping experiment of living MDCK II cells. (Left) AFM height image. First an image is scanned to define the area for spectroscopy experiment. Force distance curves are taken continuously at each pixel. (Center) Slope of the force distance curves. (Left) Hertzian mechanical model is fitted to force curves yielding a Young's modulus map.

Figure 30: Fluorescence micrograph of a FITC-concanavalin A coated MLCTcantilever to visualize coating efficiency. Scale bar $10 \mu \mathrm{m}$.

Figure 31: A: Impedance as a function of frequency obtained from a frequency scan measurement for an electrode covered with confluent MDCK II cells. B: Schematic illustration of the ECIS contact area model introduced by Giaever and Keese. Cells are represented by insulating particles with a certain distance to the gold electrode. The solid arrow indicates the current passing the intercellular gap $\left(R_{b}\right)$, while the curved arrow marks the current flowing under the cells in the adhesion zone represented by the parameter $\alpha$. The broken arrow shows the transcellular current flow at high frequencies $>10 \mathrm{kHz}$ mirrored by the capacitance $C_{m}$. C: Simplified equivalent circuit diagram describing an electrode covered with a confluent cell monolayer. $R_{b u l k}$ represents the ohmic resistance of the electrolyte and CPE (constant phase element) accounts the fractal impedance of the electrode-electrolyte interface.

Figure 32: Surface characterization of confluent MDCK II cells. A: AFM deflection image. B: Fluorescence micrograph of F-actin and (C) ezrin staining, demonstrating co-localized structures in the apical part of the cell. D: SEM image of the surface of MDCK II cells. Clearly visible are membrane protrusions.

Figure 33: Fluorescence micrographs of MDCK II cells showing changes in cortical structure during cell division. A: Ezrin staining. B: F-actin. C: Cell nucleus. Scale bar is $10 \mu \mathrm{m}$.

Figure 34: Mechanical investigation of confluent MDCK II cells. A: AFM height image. B: Young's modulus map obtained from fitting the model to force distance curves as described in section 3.1. C: Phase contrast micrograph of the same position as in A and B. In the upper part of the image the cantilever is visible. D: Histogram of Young's modules obtained from force map in B.

Figure 35: A: Three-dimensional AFM height image of confluent MDCK II cells. Line scan represents the first line in the 3D image. The apical membrane is substantially roughened due to the presence of microvilli. B: Schematic drawing illustrating modeling of MDCK II cells as spherical caps limited by cell-cell contacts.

Figure 36: Results of force cycle measurements to assess the mechanical properties of the epithelial cell line MDCK II. A: Histogram of tension values $T_{0}$ obtained from indentation experiments $(n=160)$. B: Histogram of tension values $T_{t}$ calculated from tether pulling experiments using $(n=750)$. C: Histogram of 
apparent area compressibility module $\tilde{K}_{\mathrm{A}}$ showing a bimodal distribution ( $n=$ 160). The dotted red line represents a Gaussian fit of peak I and II.

Figure 37: Analysis of fluorescence micrographs of cellular apex of MDCK II cells. (A) Cells are labeled against ezrin (green) and nucleus (blue). (B) Region of interest from micrograph (A) (100x100 pixel). Green fluorescence displays the protein ezrin. (C) White pixels correspond to green staining in (B). Analysis shows a distribution of white to black pixels of $32 \%$ to $68 \%$.

Figure 38: Tether force represented as boxplots plotted against different pulling velocities. Dashed line represent linear fit giving the linear equation $F=51.4 \cdot 10^{-11}$ $\mathrm{N}+3.53 \cdot 10^{-6} \mathrm{Ns} \mathrm{m}-1 \cdot v$.

Figure 39: Schematic illustration of a pulled membrane nanotube.

Figure 40: Time resolved ECIS measurement of MDCK II cell treated with MBCD analyzed by applying model based on Lo and Ferrier [129]. A: Parameter $\alpha$ describing current passing paracellular space decreases synchronously with $R_{b}$. B: Barrier resistance $R_{b}$ decreases after addition of MBCD to a final concentration of $10 \mathrm{mM}$. C: Specific membrane capacity $C_{m}$ shows a retarded declining effect after cholesterol depletion. Time point $0 \mathrm{~h}$ indicates MBCD addition.

Figure 41: Fluorescence micrographs of MDCK II cells. In green the cellsubstrate adhesion protein integrin is shown (A-D) and tight junction protein $\mathrm{ZO}$ 1 (E-F) after different exposure times to MBCD. A and E 0h, B and F 1h, C and G 2h, D and H 3h. Scal bar $10 \mu \mathrm{m}$.

Figure 42: Effect of cholesterol depletion by MBCD, on the properties of the plasma membrane of MDCK II cells. Immunostaining of ezrin (green, A) and Factin (red, B) and corresponding AFM deflection images (C), taken at different exposure times of MBCD. Untreated cells $(0 \mathrm{~h})$ display a large number of microvilli at the plasma membrane, which is reduced after $2 \mathrm{~h}$ of incubation with MBCD. After $3 \mathrm{~h}$ only few microvilli remain visible at the apical membrane of the cells. Scale bar: $10 \mu \mathrm{m}$.

Figure 43: SEM image of MDCK II cells after exposure to cholesterol depleting MBCD for 3h. Scale bar $2 \mu \mathrm{m}$.

Figure 44: Membrane staining of living MDCK II cells to visualize membrane uptake after MBCD treatment. Focal plane is in the lower part of the cell (basal). A-B: Untreated cells to show normal endocytotic uptake rate. C-D Cells before and after $3 \mathrm{~h}$ treatment with MBCD. Green fluorescence displays membraneuptake during cholesterol depletion, small vesicular structures are detectable in lower part of the cell (D).

Figure 45: Parameter derived from ECIS measurements of MDCK II cells with different exposure times to MBCD (10 mM) and removal of MBCD after 1(lower row), 2 (middle row) and 3h (upper row). Dotted lines indicate point of substance addition. Time point 0 marks medium exchange. Grey area highlights the recovery 
period until the respective parameter regains its initial value prior to MBCD treatment.

Figure 46: Changes of the Young's modulus of MDCK II cells during exposure to cholesterol depleting MBCD. A: Young's modulus map of confluent MDCK II cells before treatment. B: Young's modulus map during MBCD treatment. Force map starts in the lower left corner 15 min after substance addition. C: The Young's modulus increases with progressing cholesterol depletion. Gray bars display Young's modulus of untreated cells in A. Black bars show Young's modulus during cholesterol depletion.

Figure 47: Effect of cholesterol depletion by MBCD, on the properties of plasma membrane mechanics of MDCK II cells. A: Membrane tension $T_{0}, T_{t}(\mathrm{~B})$ and apparent area compressibility modulus (C) of MDCK II cells after cholesterol depletion from the plasma membrane.

Figure 48: Fluorescence micrographs of MDCK II cells labeled for F-actin (red) and ezrin (green). Untreated cells (A,B) and cytochalasin D treated cells (C, D). Scale bar $20 \mu \mathrm{m}$.

Figure 49: Mechanical investigation of MDCK II cells after disrupting the cellular cortex with cytochalasin D. A: Area compressibility modulus. B: Histogram of the area compressibility modulus, gray bars demonstrates untreated cells. C: Membrane tension $T_{0}$ and $T_{t}$ (D). E: Young's modulus. F: Tether length.

Figure 50: Fluorescence micrographs of MDCK II cells treated with blebbistatin. A: Ezrin is labeled in green showing protein distribution at the cellular apex. B: Factin is labeled in red. C: F-actin in the basolateral region of the cells.

Figure 51: Schematic illustration of the uncoupling of the plasma membrane from the cytoskeleton by neomycin injection due to binding of neomycin to $\mathrm{PIP}_{2}$.

Figure 52: Microinjection of neomycin into a single MDCK II cell of a confluent monolayer. A: AFM deflection image showing substantial alteration in plasma membrane topography $4 \mathrm{~h}$ after neomycin injection (loss of microvilli). The line profile taken along the dotted line shows that the cell height of the injected cell is reduced compared to its adjacent neighbors. Scale bar: $5 \mu \mathrm{m}$. B: Zoom-in of A showing details of the surface features of the injected cell. The cellular cortex bulges beneath the plasma membrane as highlighted by white markers. C: Surface details of adjacent untreated cell (control). E: The injected cell is negative for immunostaining of ezrin. D: Fluorescence image of co-injected FITC-dextran for the localization of the injected cell.

Figure 53: Topological features of the plasma membrane of MDCK II cells. A: Deflection image of neomycin injected cell. B: SEM image of TriX treated MDCK II cells, exposing the terminal web under the dissolved plasma membrane. Scale bar in A is $1 \mu \mathrm{m}$ in B $0.1 \mu \mathrm{m}$. 
Figure 54: Analysis of neomycin injected cell (4h after injection) with the Hertzian approach: A: Young's modulus map. Scale bar $10 \mu \mathrm{m}$. B: E-values of adjacent untreated cells and injected one.

Figure 55: Mechanical parameters $\left(\tilde{K}_{\mathrm{A}}, T_{0}\right.$ and $T_{\mathrm{t}}$ ) after injection of neomycin. (** correspond to $\mathrm{p}<10^{-2}$; $* * *$ to $\mathrm{p}<10^{-3}$, two-tailed Wilcoxon rank sum test). D: Boxplot of tether lengths from tether pulling experiments before and after the injection of neomycin.

Figure 56: A: AFM deflection image of neomycin injected MDCK II cell $4 \mathrm{~h}$ after injection. Scale bar $10 \mu \mathrm{m}$. Inlay shows the fluorescence micrograph of the coinjected FITC-dextran. B: Fluorescence micrograph of the immunostaining of ezrin of a neomycin injected MDCK II cell.

Figure 57: Mechanical parameter obtained from indentation experiments of a neomycin injected MDCK II cell. Area 1 corresponds to untreated adjacent cells. Area 2 belongs to regions on the injected cell where still ezrin protein is detectable. Region 3 is mainly devoid of ezrin. (** correspond to $\mathrm{p}<10^{-2}$; *** to $\mathrm{p}<10^{-3}$, two-tailed Wilcoxon rank sum test).

Figure 58: Cellular reaction of injection of neomycin. Cells frequently undergo apoptosis. A: Phase contrast micrograph of neomycin injected MDCK II cells (1h). B: Fluorescence micrograph of neomycin injected cells. The cells are coinjected with FITC-dextran. C: Phase contrast micrograph of neomycin injected cell after 6h. Cell in the lower right corner is apoptotic. D: Fluorescence micrograph of cell in C. Scale bar $10 \mu \mathrm{m}$.

Figure 59: Cellular reaction of injection of neomycin. A: Phase contrast micrograph of neomycin injected MDCK II cells (1h). B: Fluorescence micrograph of neomycin injected cells. C: Phase contrast micrograph $8 \mathrm{~h}$ after injection. D: AFM deflection image of injected cell (8h). Scale bar $10 \mu \mathrm{m}$.

Figure 60: A: AFM deflection image of MDCK II cells after neomycin injection. Green arrow mark injected cell, white arrows show membrane strains pulled out of the plasma membrane of injected cells by a ConA coated cantilever tip. Scale bar $10 \mu \mathrm{m}$. B: Fluorescence micrograph of neomycin injected cell. Cells are labeled for ezrin. Injected cells display decreased level of ezrin. Scale bar $10 \mu \mathrm{m}$.

Figure 61: Topographical alteration of confluent MDCK II cells after injection of $\mathrm{PIP}_{2}$ - micelles into a single cell. A: AFM deflection image after $\mathrm{PIP}_{2}$ injection. Red dashed line display injected cell, white untreated one. B: Line scan of A. C Three dimensional reconstruction of the AFM height image in A from untreated cell. Membrane protrusions are visible. D: 3-D reconstruction from height image in A of injected cell.

Figure 62: Fluorescence micrographs of MDCK II cells after PIP $_{2}$ injection. A and $\mathrm{C}$ : Immunostaining of $\mathrm{PIP}_{2}$ after microinjection. Arrow marks the injected cell. Scale bar is $20 \mu \mathrm{m}$. B: Ezrin of injected and untreated cells are immunostained with Alexa Fluor 488. D: F-actin cytoskeleton is stained with Alexa Fluor 488. 
Figure 63: Mechanical investigation of MDCK II cells after injecting $\mathrm{PIP}_{2^{-}}$ micelles, at different time points after injection. A: Membrane pre-tension $T_{0}$. B: Apparent area compressibility modulus.

Figure 64: Microinjection of FITC-dextran into confluent MDCK II cells. A: Fluorescence micrograph of FITC-dextran injected cells. B: AFM-deflection image after injection. C: Young's modulus map after microinjection of FITCdextran. Scale bar $10 \mu \mathrm{m}$.

Figure 65: Evaluation of mechanical changes due to microinjection. A: Young's modules, B: Membrane tension, C: Apparent area compressibility modulus of FITC-dextran injected cells and untreated one.

Figure 66: Illustration of apical membrane sheet preparation on porous substrate. A-B: Confluent MDCK II cells are hypoosmotically shocked prior to membrane deposition. C-D: A poly-D-lysin coated porous chip is gently pressed on the apical surface of the cells. By lifting the chip off the cells, the apical membrane is transferred to the porous substrate.

Figure 67: A: Force-distance approach curves taken on the rim (blue) or on a pore covered with membrane (black). B: AFM height images of apical membrane sheets on a porous substrate. C: Calculated shape (blue line) of a membrane indented by a conical or pyramidal indenter (black line) assuming a lateral tension $T_{0}$ of $0.16 \mathrm{mN} / \mathrm{m}$ and a bending modulus $\kappa$ of $25 k_{\mathrm{B}} T$. The shape of the membrane is essentially identical to that of a point-like indenter justifying the use of equation (35) to fit the indentation data. D: Fluorescence micrograph of apical membrane sheets labeled with DiI(18) on a porous substrate.

Figure 68: Morphological changes of confluent MDCK II cell after osmotic stress. A-D 3D-height images of confluent MDCK II cells treated with different osmotic solutions. A: isotonic. B: 200 mOsm. C: 80 mOsm. D: 700 mOsm. Height of the Box is $5 \mu \mathrm{m}$, box length and width is $50 \mu \mathrm{m}$. E-H: AFM deflection images, scale bar is $10 \mu \mathrm{m}$. E: untreated cell. F: $200 \mathrm{mOsm}$. G: 80 mOsm. H: 700 mOsm. I: Cell height and J: Cell radius, extracted from AFM height images, at least $n=10$ cells. $\mathrm{K}$ : Schematic drawing of spherical caps to demonstrate change in cell size due to osmotic treatment.

Figure 69: Indentation experiments revealing mechanical information of hypoosmotic stressed confluent MDCK II cells. A: Representative force distance traces taken on treated cells. Trace curve is used for determine the area compressibility module and tension $T_{0}$ using a modified liquid droplet model for indentation experiments fitted to the experimental data. B: Area compressibility modulus, reflecting the changes in membrane area during the treatment. C: Changes in membrane tension $T_{0}$ by altering the osmotic stress or F-actin integrity by cytochalasin $\mathrm{D}$.

Figure 70: Membrane Tension $T_{t}$ calculated from tether pulling experiments. For computing $T_{t}$ a bending module $\kappa$ of $10^{-19} \mathrm{~J}$ representing fluid lipid bilayer is assumed. Viscous contributions to the tether force are neglected as a first approximation [110, 170]. 
Figure 71: Histogram of tether forces obtained from retrace curves of a force cycle of tether pulling experiments, with corresponding fluorescence micrographs of different treated MDCK II cell. A: Histogram of tether forces of untreated cells (isotonic, $308 \mathrm{mOsm}$ ). A1: shows apical part of the cell, A2 the corresponding basolateral part. B: 200 mOsm. C: 80 mOsm. D: Cells treated with F-actin destabilizing cytochalasin. Scale bar is $20 \mu \mathrm{m}$.

Figure 72: Mechanical changes resulting from long incubation in hypoosmotic solution. A: Apparent area compressibility modulus at different time points. B: Membrane tension obtained from indentation experiments. C: Lorenz-Fits of tether force histogram displaying change in tension during regulatory process. D: Membrane Tension $T_{t}$ calculated from tether pulling experiments.

Figure 73: Fluorescence micrograph of confluent MDCK II cells challenged $2 \mathrm{~h}$ in strong hypoosmotic solution (80 mOsm). F-actin is labeled in green. Scale bar 10 $\mu \mathrm{m}$.

Figure 74: Fluorescence micrographs of confluent MDCK II cells challenged for 0h (A), 2h (B) and 6h (C) in strong hypoosmotic solution (80 mOsm). Microfilament- membrane anchor protein ezrin is labeled in green, F-actin in red. Scale bar is $20 \mu \mathrm{m}$.

Figure 75: A: Histograms of tether forces obtained from experiments on hypertonic challenged cells compared to a control (700 mOsm 308 mOsm). Fluorescence micrographs of F-actin labeled MDCK II cells treated with isotonic (A1: apical, A2: basal) and hypertonic (B1: apical, B2: basal) solution for $30 \mathrm{~min}$. Scale bar is $20 \mu \mathrm{m}$. C: Membrane tension $T_{t}$ estimated from tether pulling experiments.

Figure 76: Phase contrast micrograph of MDCK II cells exposed to distilled water. A: untreated cells. B: treated cells. Whit arrow in right corner of the image indicates ruptured cell. Scale bar $20 \mu \mathrm{m}$.

Figure 77: Mechanical investigation of MDCK II cells under different osmotic stress A: Force distance curves recorded on MDCK II cells after different treatments. (๑) Green dots display mechanical response of untreated cells to indentation. (•) Black dots cells during hyperosmotic stress. (•) Red dots mark cells exposed to distilled water. B: Young's modulus of cells treated with very low osmolarity (distilled water) ( $\mathrm{n}=5$ cells).

Figure 78: Schematic illustration of osmotic challenged MDCK II cells. A: Cell shrinkage due to hyperosmotic stress acting outwards on the plasma membrane. $\mathrm{B}$ : Cells under isotonic conditions exposing membrane reserves and underlying cortex. C: Cell swelling due to osmotic pressure acting against the internal plasma membrane (hypoosmotic conditions).

Figure 79: Mechanical properties of MDCK II cell during adhesion and spreading. A: Force curves taken at different time point during the spreading process. Force curve taken 5 min after cell seeding (open circle) shows the steepest slope 
followed by a sudden softening (10 min, black circle). Within $30 \mathrm{~min}$, force curves snap back to higher stiffness. B:Slope of FCs plotted against time.

Figure 80: A-D Light microscopy images taken at different time points documenting the spreading behavior of MDCK II cells. E: The adhering and spreading cell is locally indented with an AFM tip resulting in force curves shown exemplary in Figure 79. While retracting the cantilever a membrane nanotube (tether) is frequently pulled out of the plasma membrane bearing additional mechanical information.

Figure 81: Phase contrast micrographs of MDCK II cell in suspension. Cells display membrane protrusions, indicated by white arrows. Scale bar is $10 \mu \mathrm{m}$.

Figure 82: Mechanical model is changed from a sphere to a caped sphere. (Right) Force curves taken on a spherical cell (dots) and spreading cell (squares) with the corresponding fit using membrane theory (section 3).

Figure 83: Mechanical parameter obtained from fitting the liquid droplet model for spherical (๑) or adherent cell $\left((\square)\right.$ for $\phi=75^{\circ},(\Delta)$ for $\left.\phi=21^{\circ}\right)$ to experimental data obtained from adhesion and spreading process of a MDCK II cell.

Figure 84: Mechanical parameter of adhering and spreading MDCK II cells ( $\mathrm{n}=$ 4). Apparent area compressibility modulus and membrane tension $\left(T_{0}\right.$ and $\left.T_{T}\right)$ are monitored during spreading progress. Circles correspond to model for spherical cells, while squares represent mechanical parameters obtained from model of an adhering cells with $\phi_{0}=75^{\circ}$. Triangles represent modeling of a spread cell with $\phi_{0}$ $=21^{\circ}$. C: Membrane Tension $T_{t}$ calculated from tether pulling experiments.

Figure 85: Phase contrast micrograph of full spread MDCK II cell 24h after cell seeding.

Figure 86: Fluorescence micrographs of F-actin stained spherical MDCK II cell 5 min after cell seeding (left column), an adhering cell 15 min after cell seeding (middle column) and spread one 30 min after cell seeding (right column). One column displays slices through the cell starting from the upper part. It should be pointed out, that 10 min after cell seeding, F-actin distribution changes. The prior uniform actin layer (cortex), as observed for a cell after 5 min, disappears in the basal part and membrane blebs start to form.

Figure 87: Fluorescence micrographs of a spherical MDCK II cell (membrane staining) 5 min after cell seeding (left column), an adhering cell $15 \mathrm{~min}$ after cell seeding (middle column) and a spread cell 30 min after seeding (right column). Left column shows slices through a spherical cell, beginning at the top. Middle column shows corresponding images through an adhering cell, and right column through a spreading cell. Scale bar $20 \mu \mathrm{m}$.

Figure 88: Phase contrast image of a spherical, non-spread MDCK II cell on BSA coated surface. In left corner the cantilever is visible. Scale bar $10 \mu \mathrm{m}$. B: Force distance curves taken over time on a non-spreading MDCK II cell. Slope of FC 
does not change over time. For comparison, a FC of adhering and spreading cell is plotted (in black).

Figure 89: Illustration of the key events in cellular adhesion and spreading deduced from mechanical experiments as a function of time. (1.) Detachment of an adherent cell due to trypsinization. Freshly detached cells restore tension by creating actin-coated protrusions that consume excess membrane to maintain tension (2.). Due to adhesion, the actin cortex partially opens close to the substrate (3.) allowing a bulk flow of cytoplasma to cause blebs as an onset of spreading (4.). In the last step cells start to flatten and the cell's surface becomes smoother while stress fibers form (5.). 


\section{List of Tables}

Table1: Mechanical characteristics of the three cytoskeleton building filaments [1].

Table 2: Techniques used to study cellular mechanics [72]

Table 3: Objectives and light source used for microinjection experiments

Table 4: Osmotic solutions:

Table 5: Antibodies and reagents for immunostaining.

Table 6: Mechanical parameter for calculating the bending rigidity $\kappa . T_{0}$ is obtained from indentation experiments.

Table 7: ECIS parameter from experiment shown in Figure 41.

Table 8: Young's modules of MDCK II cells during cholesterol depletion. For time point 15 min line 1 from force map in A or B is averaged $(n=32)$, etc. Data are presented as mean values with standard deviation.

Table 9: Mechanical parameters of confluent MDCK II cells obtained from force cycle analysis after exposure to MBCD. Values represent the maximum of a Gaussian curve fitted to the histograms.

Table 10: Mechanical parameter after cell treatment with blebbistatin. Mean values are represented with standard deviation.

Table 11: Parameters for modeling spherical cap describing confluent MDCK II cells when subjected to osmolar stress.

Table 12: Mechanical parameter obtained from long incubation in hypoosmotic solution. Values are representing the median from boxplots in Figure 72 and the median absolute deviation.

Table 13: Parameters for modeling spherical cap describing confluent MDCK II cells, when subjected to hypotonic stress (80 mOsm) over $6 \mathrm{~h}$.

Table 14: Mechanical parameters obtained from hypertonic stress on confluent MDCK II cells. 
